\title{
N-linked glycosylation of ether á go-go potassium channels: effects on cell surface expression and functional properties
}

\author{
PhD Thesis \\ In partial fulfilment of the requirements \\ For the degree of Doctor of Philosophy (PhD) \\ In the graduate program Neurosciences \\ At the Georg-August University Göttingen \\ Faculty of Biology
}

submitted by

Joanna Napp

Bydgoszcz, Poland

Göttingen 2003 
Advisor, first member of FAC: $\quad$ Prof. Dr. Walter Stühmer Second member of FAC: Third member of FAC: Prof. Dr. Reinhard Jahn Dr. Michael Rickmann

Date of submission of the PhD Thesis: May $26^{\text {th }} 2003$

Date of Thesis Defence (Disputation): July $3^{\text {rd }} 2003$ 


\section{SUMMARY}

Ether á go-go (Eag1) is a voltage gated potassium channel, apparently involved in a broad variety of cellular events. Results from this work show that in rat tissues the Eag1 protein is predominantly expressed in brain tissue where it undergoes $\mathrm{N}$-linked glycosylation, a cotranslational modification known to affect biogenesis and functional properties of numerous plasma membrane proteins. Analogously, human Eag1 undergoes N-linked glycosylation when expressed in heterologous systems.

The human Eag1, as expressed in CHO cells, and the rat Eag1 natively expressed in brain, each exist as two isoforms which harbour different asparagine-linked oligosaccharides. Examination of the carbohydrate attachments with endoglycosidases revealed two distinct complexes, the core-oligosaccharide and the complex-oligosaccharide, which contribute to the molecular masses of the hEag1 isoforms with 2-5 kDa and 20-25 kDa, respectively.

Mutation of the asparagine residues in two of the six putative N-linked glycosylation motifs (N-X-S/T), Asn 388 and $\mathrm{Asn}_{406}$ altered the glycosylation of hEag1 channels. However, single amino acid exchanges in the four remaining glycosylation motifs had no effect on hEag1 glycosylation.

Asparagine 388 is the site of attachment of the core-oligosaccharide, which is sensitive to Endoglycosidase $\mathrm{H}$ and recognisable by Concanavalin A. Mutation of this site had no significant effect on the intracellular localisation and functional properties of hEag1 channels, although interference with some other unidentified function cannot be excluded. Asparagine 406 carries the complex-oligosaccharide, which is resistant to Endoglycosidase $\mathrm{H}$ and unrecognisable by Concanavalin A. The attachment of the complex-oligosaccharide seems to have an important and complex function since mutation of the Asn $\mathrm{A}_{406}$ results in an altered phenotype. Intracellular distribution of hEag1 lacking the complexoligosaccharide is strongly affected, showing a complex-perinuclear localisation instead of the widespread staining pattern. This is the case also for the hEag1 mutant lacking both glycosylation sites.

Immunofluorescence experiments indicate that channel proteins lacking the complexoligosaccharides are, at least partially, trapped in the endoplasmatic reticulum. This can be explained, e.g., by folding problems due to the loss of oligosaccharide attachment. The altered intracellular localisation of the unglycosylated and the solely core-glycosylated protein is not likely due to the introduced mutation, since the same effect was obtained by expression of the wild-type protein in glycosylation deficient cells. 
Although the unglycosylated protein is partially trapped in the endoplasmatic reticulum, significant populations of mutant channels must be expressed on the cell surface, since hEag1 potassium currents are also detectable in cells expressing unglycosylated channels, albeit with a strongly reduced amplitude. Loss of the core-oligosaccharide only has no significant effect on hEag1 current amplitude.

Furthermore, loss of the complex-oligosaccharide results in a shift of the voltage-dependence towards more positive potentials, a phenomenon commonly observed in the case of desialylation of voltage-dependent sodium channels.

Additionally PIAS1 was identified as a putative hEag1 interaction partner of the C-terminus of hEag1 by two independent Yeast-Two-Hybrid screens. PIAS1 is expressed in nuclei where it acts as inhibitor of STAT mediated gene-activation. The hypothesis that a membrane protein, or at least part of it, can functionally interact with a nuclear protein is controversial, although recently evidence for such interactions are accumulating. This is of particular interest for Eag1, given its involvement in cancer. 


\section{CONTENTS}

ABBREVIATIONS......................................................................................... VI

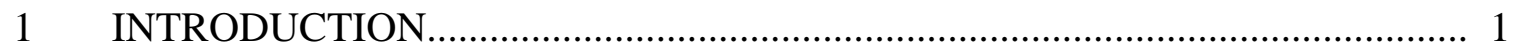

1.1 Ion channels: focus on voltage gated potassium channels................................ 1

1.2 Ether á go-go potassium channel................................................................ 3

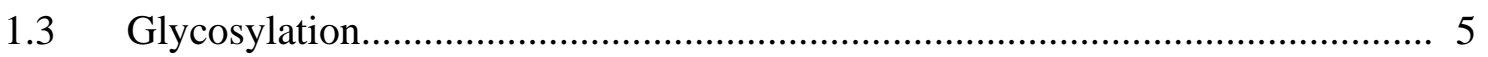

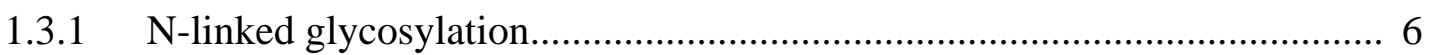

1.3.2 Other glycosylation forms (O-linked glycosylation, glypiation, C-mannosylation and phosphoglycosylation)......................................... 8

1.3.3 Function of N-linked glycosylation...................................................... 9

$1.4 \quad \mathrm{~N}$-linked glycosylation and ion channels................................................... 11

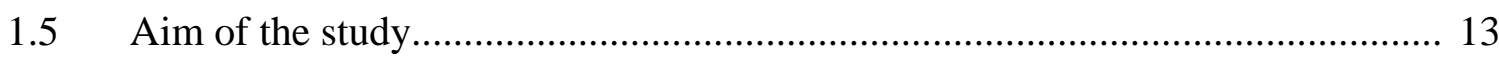

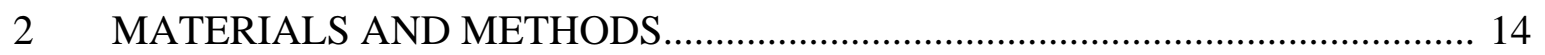

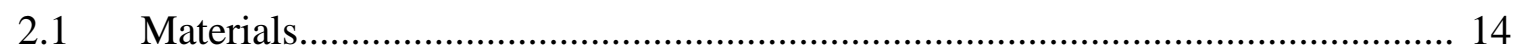

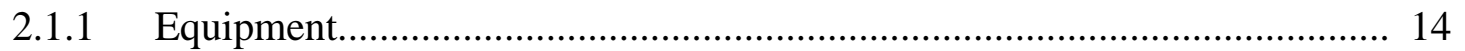

2.1.2 Chemicals, reagents and material........................................................... 14

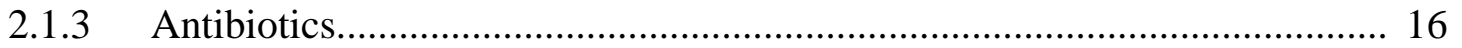

2.1.4 Enzymes, inhibitors, substrates........................................................... 16

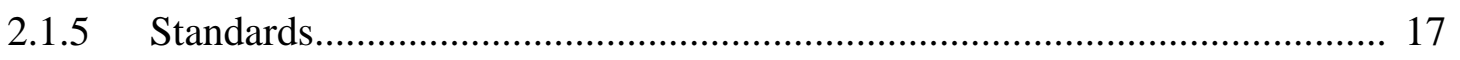

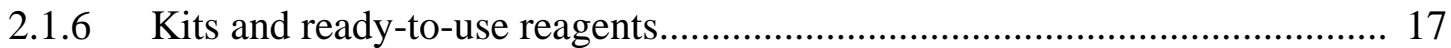

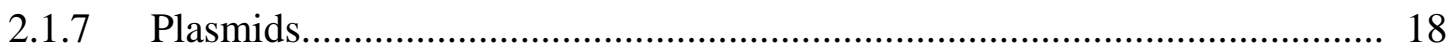

2.1.8 Synthetic oligonucleotide primers.......................................................... 19

2.1.8.1 Sequencing and PCR primers............................................................................... 19

2.1.8.2 Oligonucleotides introducing mutation................................................................. 20

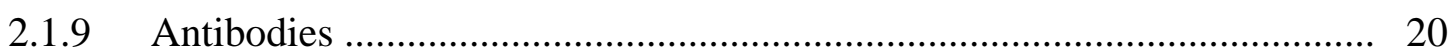

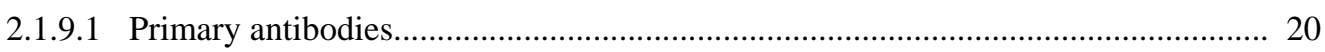

2.1.9.2 Secondary antibodies..................................................................................... 22

2.1.10 Organisms and growth media............................................................. 22 
2.1.10.1 Bacterial strain genotypes................................................................................. 22

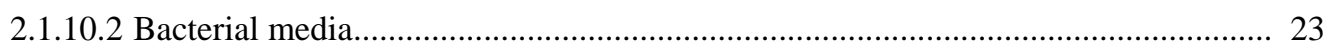

2.1.10.3 Yeast strain genotypes...................................................................................... 24

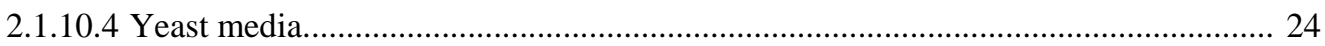

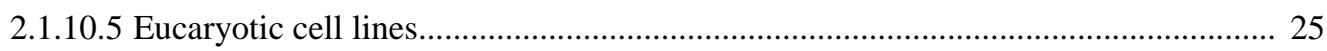

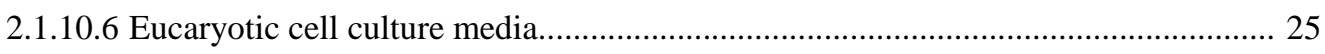

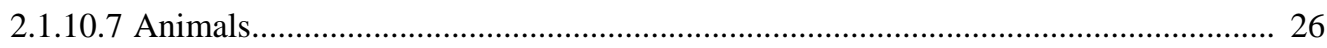

2.1.11 Sterilisation of solutions and equipment............................................... 26

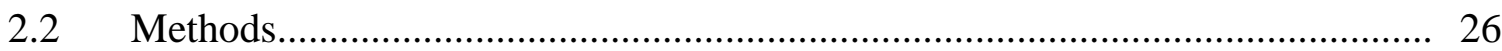

2.2.1 Bacteria and yeast culture techniques................................................. 26

2.2.1.1 Preparation of Epicurian Coli XL1-Blue competent bacteria................................. 27

2.2.1.2 Transformation of competent bacteria............................................................ 27

2.2.2 Nucleic acid preparation, modification, amplification and analysis........... 28

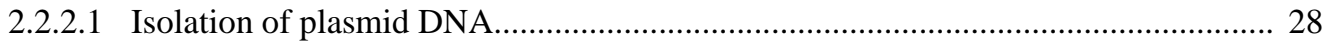

2.2.2.1.1 Small-scale preparation of plasmid DNA (Mini prep) ................................. 28

2.2.3.1.2 Large-scale preparation of plasmid DNA (Maxi prep) ............................... 28

2.2.2.2 Isolation of DNA fragments from agarose gels................................................ 28

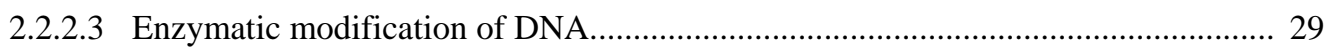

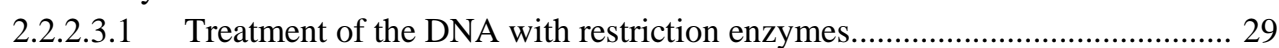

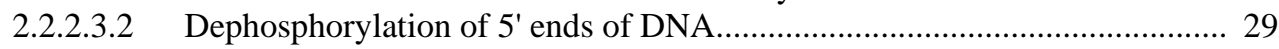

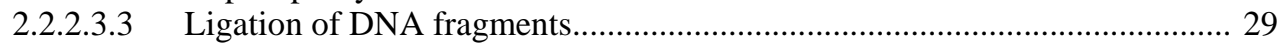

2.2.2.4 Isolation of total RNA from tissues and eucaryotic cell lines................................. 30

2.2.2.4.1 Isolation with Total RNA Isolation Reagent................................................ 30

2.2.2.4.2 Isolation of total RNA with StrataPrep ${ }^{\mathrm{TM}}$ Total RNA Microprep Kit............ 30

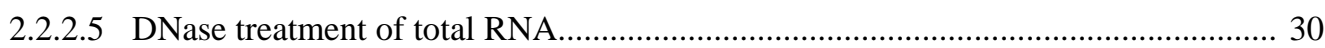

2.2.2.6 Phenol-chloroform extraction and ethanol precipitation...................................... 31

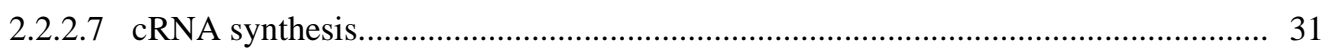

2.2.2.8 Spectrophotometric quantitation and estimation of nucleic acid purity.................. 32

2.2.2.9 RiboGreen ${ }^{\mathrm{TM}}$ assay for estimation of cRNA concentration................................. 31

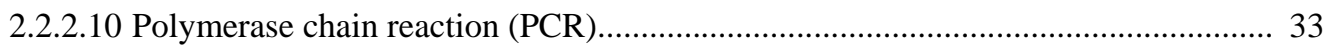

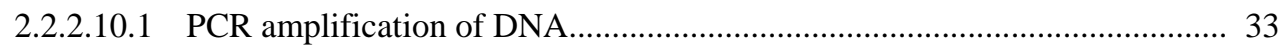

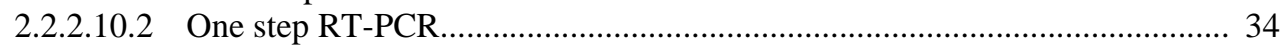

2.2.2.10.3 Site directed in vitro mutagenesis............................................................ 34

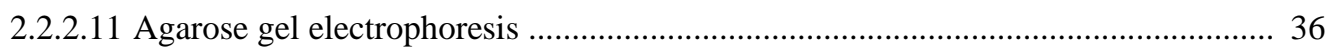

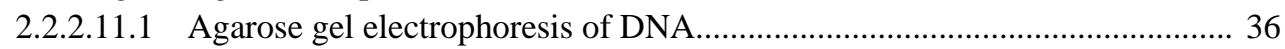

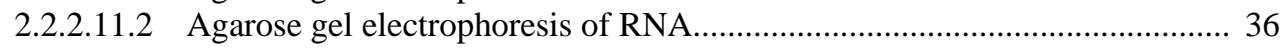

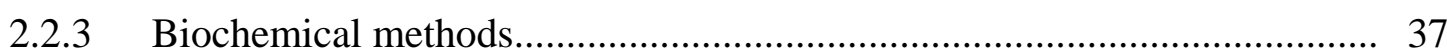

2.2.3.1 Isolation of total proteins from mammalian cells................................................... 37

2.2.3.2 Determination of protein concentration............................................................. 38

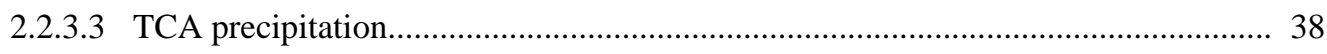

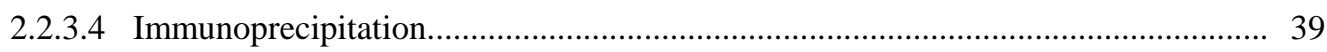

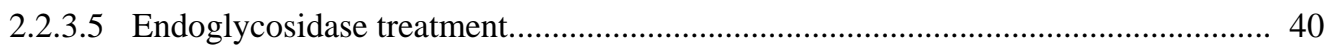

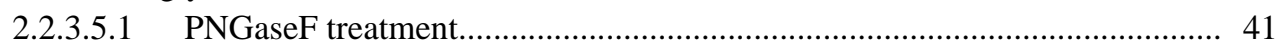

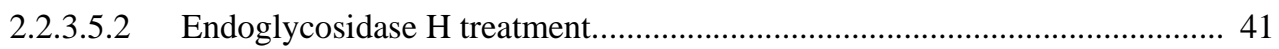

2.2.3.6 Polyacrylamide gel electrophoresis (SDS-PAGE)............................................ 41 
2.2.3.7 Commasie brilliant blue staining of proteins in polyacrylamide gels.

2.2.3.8 Western blotting and immunological detection of proteins on nitrocellulose filters

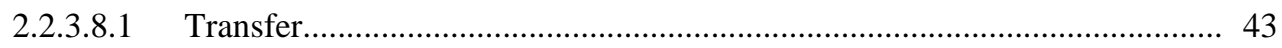

2.2.3.8.2 Ponceau staining.................................................................................. 44

2.2.3.8.3 Antibody detection and enhanced chemiluminescent (ECL) assay for protein visualisation....................................................................... 44

2.2.3.9 Subcellular fractionation................................................................................ 45

2.2.3.9.1 Fractionation by differential centrifugation............................................... 45

2.2.3.9.2 Subcellular fractionation by velocity sedimentation on sucrose gradient........... 46

2.2.4 Eukaryotic cell culture methods.................................................... 47

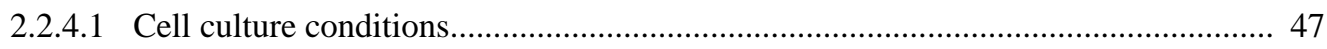

2.2.4.2 Harvesting eucaryotic cells............................................................................... 47

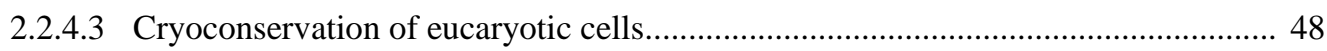

2.2.4.4 Thawing and revitalising of cryopreserved cells............................................... 48

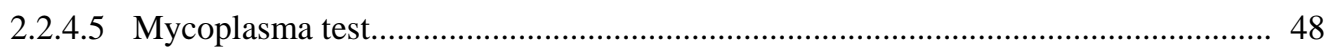

2.2.4.6 Transfection of mammalian cells....................................................................... 49

2.2.4.7 Preparation of polylysine-coated tissue culture surfaces........................................ 49

2.2.4.8 Incubation with Tunicamycin.................................................................... 50

2.2.5 Microscopic analysis............................................................ 51

2.2.5.1 Immunofluorescent localisation of proteins................................................... 51

2.2.6 Electrophysiological methods.................................................. 51

2.2.6.1 Preparation and injection of Xenopus laevis oocytes......................................... 52

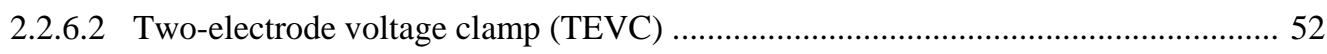

2.2.6.3 TEVC stimulation protocols........................................................................... 53

2.2.6.4 Patch clamp measurements of potassium currents from $\mathrm{CHO}$ cells........................ 55

2.2.6.5 Stimulation-acquisition protocols and data analysis......................................... 56

2.2.7 Yeast Two-Hybrid System $(\mathrm{Y} 2 \mathrm{H})$........................................................... 57

2.2.7.1 Transformation of S.cerevisiae AH 109........................................................... 58

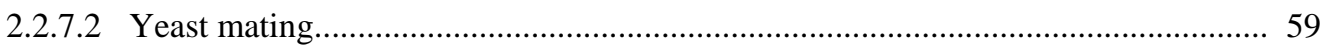

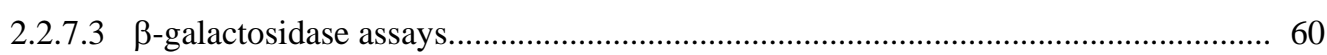

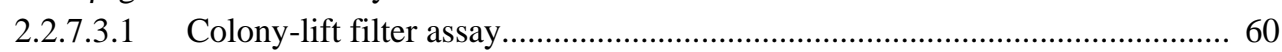

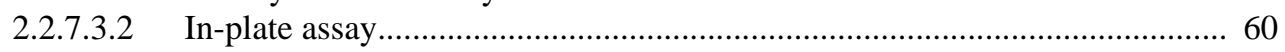

2.2.7.4 Recovery of plasmid DNA from S. cerevisiae.................................................... 61

2.2.7.5 Isolation of total cellular proteins from S.cerevisiae AH 109............................... 62

2.2.8 Computer analysis..................................................................... 62

$3 \quad$ RESULTS

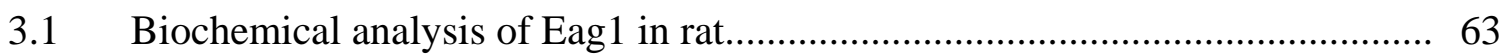

3.1.1 Analysis of the Eag1 protein expression pattern in rat brain......................63 63

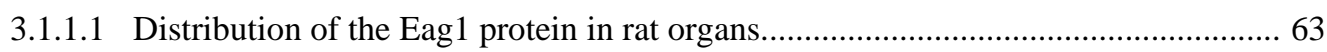

3.1.1.2 Immunoprecipitation of the Eag1 protein from brain homogenates........................ 65 
3.1.2 Glycosylation of th Eag1 in rat brain.

3.2 Biochemical and functional characterisation of human Eag1

expressed in heterologous systems.

3.2.1 Expression of hEag1 in CHO cells........................................................ 69

3.2.2 Analysis of hEag1 sensitivity to endoglycosidases.................................... 70

3.2.1 Disruption of the hypothetical glycosylation sites by site directed

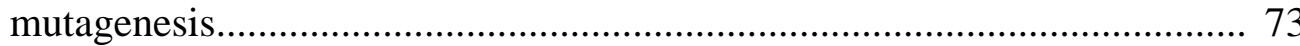

3.2.4 Selection of the mutant phenotypes with enzymatic deglycosylation.......... 75

3.2.5 Generation and biochemical analysis of the double glycosylation mutant... 76

3.2.6 Model of the N-linked glycosylation of hEag1 potassium channel.............. 78

3.2.7 Binding of hEag1-linked oligosaccharides to Concanavalin A................... 81

3.2.8 Distribution of hEag1 glycosylation isoforms in subcellular fractions........ 82

3.2.8.1 Fractionation by differential centrifugation................................................................. 83

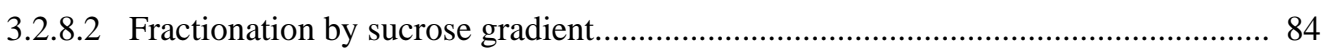

3.2.9 Effects of the N-linked glycosylation on intracellular distribution of hEag1 potassium channels..................................................................... 86

3.2.9.1 Intracellular localisation of the hEag1 and hEag1 mutant proteins (hEag1-N388Q, -N406Q and -N388,406Q) in CHO cells..................................... 86

3.2.9.2 Characterisation of hEag1 expression in glycosylation deficient cells.................... 89

3.2.9.2.1 Internal block of the N-linked glycosylation of hEag1 in CHO cells

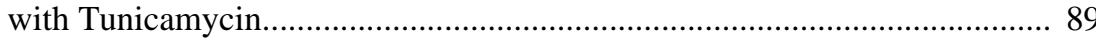

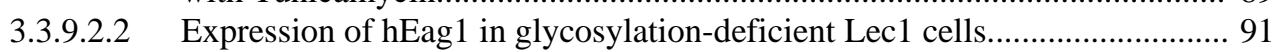

3.2.9.2.3 Colocalisation of hEag1 and mutant proteins with different intracellular markers 93

3.2.10 Functional characterisation of hEag1 and glycosylation deficient

hEag1 potassium channels 100

3.2.10.1 Heterologous expression of the hEag1 and glycosylation mutants

in Xenopus laevis oocytes. 100

3.2.10.2 Voltage dependence of hEag1-N406Q mutant channels. 102

3.2.10.3 Functional characterisation of the glycosylation deficient hEag1 .

in mammalian cells 103

3.2.11 Identification of putative hEag1 binding partners by Yeast

Two-Hybrid System......................................................................... 106

3.2.11.1 Cloning of the C-terminus of hEag1 into the pGBKT7 vector............................... 106

3.2.11.2 Expression of the GAL4-hEag1CT fusion protein and transactivation test............. 106

3.2.11.3 Screening of a human brain cDNA library by yeast mating.................................. 108

3.2.11.4 Analysis of the putative interaction partners of hEag1 ........................................ 108 


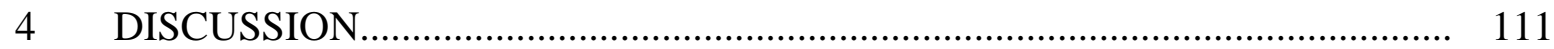

4.1 Expression pattern of Eag1 protein in rat tissues........................................... 112

4.2 N-linked glycosylation of Eag1 potassium channels..................................... 113

4.3 Model for N-linked glycosylation of human ether á go-go potassium channel.................................................................................. 116

4.4 Effects of N-linked glycosylation on the intracellular transport of hEag1........ 120

$4.5 \quad$ N-linked glycosylation and functional properties of hEag1........................... 124

4.6 Does N-linked glycosylation affect the stability of hEag1?............................ 127

4.7 What is the functional role of N-linked glycosylation of Eag1 potassium channels in native cells?................................................. 128

4.8 Can hEag1 interact with transcription regulators?...................................... 129

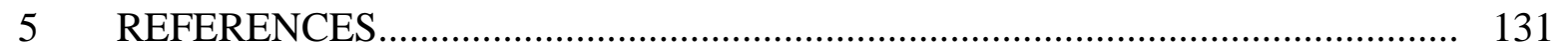




\section{ABBREVIATIONS}

\section{Units}

$\begin{array}{ll}\Omega, \mathrm{M} \Omega, \mathrm{G} \Omega & \text { Ohm, megaohm, gigaohm } \\ \mathrm{A}, \mathrm{pA} & \text { Ampere, picoampere } \\ \mathrm{bp}, \mathrm{kb} & \text { Base pair, kilobase } \\ { }^{\circ} \mathrm{C} & \text { Celsius } \\ \mathrm{Da}, \mathrm{kDa} & \text { Dalton, kilodalton } \\ \mathrm{F}, \mathrm{pF} & \text { Farad, picofarad } \\ \mathrm{G} & \text { Acceleration } \\ \mathrm{g}, \mathrm{mg}, \mu \mathrm{g}, \mathrm{ng} & \text { Gram, milligram, microgram, nanogram } \\ \mathrm{I} & \text { Current } \\ \mathrm{l}, \mathrm{ml}, \mu \mathrm{l} & \text { Liter, milliliter, microliter } \\ \mathrm{M}, \mathrm{mM}, \mu \mathrm{M}, \mathrm{nM} & \text { Molar, millimolar, micromolar, nanomolar } \\ \mathrm{Po} & \text { Open probability } \\ \mathrm{R} & \text { Resistance } \\ \mathrm{rpm} & \text { Rotations per minute } \\ \mathrm{s} & \text { Second } \\ \mathrm{U} & \text { Unit } \\ \mathrm{V}, \mathrm{mV} & \text { Volt, millivolt } \\ \text { v/v } & \text { Volume for volume } \\ \text { w/v } & \text { Weight for volume }\end{array}$

\section{Cell lines, Organisms, Genes and Proteins}

$\begin{array}{ll}\text { cDNA, cRNA } & \text { Complementary DNA, RNA } \\ \text { CHO } & \text { Chinese Hamster Ovary } \\ \text { DNA, RNA } & \text { 2-deoxy D-ribonucleic acid, ribonucleic acid } \\ \text { E.Coli } & \text { Escherichia Coli } \\ \text { Eag, hEag1 } & \text { ether á go-go, human ether á go-go } 1 \\ \text { ER } & \text { Endoplasmatic reticulum } \\ \text { Erg } & \text { Eag related gene } \\ \text { HRP } & \text { Horseradish peroxidase } \\ \text { IgG } & \text { Immunoglobulin G } \\ \text { N388,406Q } & \text { hEag1-N388,406Q; double mutant lacking core- and } \\ & \text { complex-glycosylation sites; both asparagines were } \\ & \text { exchanged to glutamines } \\ \text { N388Q } & \text { hEag1-N388Q; hEag1 mutant lacking site for attachment } \\ & \text { of the N-linked core-oligosaccharide } \\ \text { N406Q } & \text { hEag1-N388Q; hEag1 mutant lacking site for attachment } \\ & \text { of the N-linked complex-oligosaccharide } \\ \text { WT } & \text { Wild-type }\end{array}$




\section{Methods and Chemicals}

\begin{tabular}{|c|c|}
\hline $\mathrm{AD}$ & Activation domain \\
\hline $\mathrm{BD}$ & Binding domain \\
\hline BrET & Ethidium bromide \\
\hline BSA & Bovine Serum Albumin \\
\hline Con A & Concanavalin A \\
\hline $\mathrm{ddH}_{2} \mathrm{O}$ & Double distilled water \\
\hline DEPC & Diethyl pyrocarbonate \\
\hline DMSO & Dimethyl sulfoxyde \\
\hline DNase & Deoxyribonuclease \\
\hline dNTP & Deoxyribonucleoside triphosphate \\
\hline DTT & 1,4-Dithio-DL-threitol \\
\hline ECL & Enhanced chemiluminescence \\
\hline EDTA & Ethylenediamine tetraacetic acid \\
\hline Endo $\mathrm{H}$ & Endoglycosidase $\mathrm{H}$ \\
\hline $\mathrm{EtOH}$ & Ethanol \\
\hline FCS & Fetal Calf Serum \\
\hline GlcNAc & N Acetyl glucosamine \\
\hline HEPES & 4-(2-hydroxyethyl)-1-piperazine ethanesulphonic acid \\
\hline IP & Immunoprecipitation \\
\hline LB & Luria Bertani (medium) \\
\hline $\mathrm{MeOH}$ & Methanol \\
\hline MOPS & 3-(N-Morpholino) propanesulfonic acid \\
\hline MW & Molecular weight \\
\hline OD & Optical density \\
\hline PBS & Phosphate Buffered Saline \\
\hline PCR & Polymerase Chain Reaction \\
\hline PFA & Paraformaldehyde \\
\hline PNGase F & Peptide N-Glycosidase F \\
\hline RNase & Ribonuclease \\
\hline rpm & Rotations per minute \\
\hline RT-PCR & Reverse transcriptase polymerase chain reaction \\
\hline SDS & Sodium dodecyl sulphate \\
\hline SDS-PAGE & SDS-polyacrylamide gel electrophoresis \\
\hline TEMED & N’N’N’N Tetramethylethyldiamine \\
\hline TEVC & Two-Electrode Voltage Clamp \\
\hline $\mathrm{V}$ & Voltage \\
\hline $\mathrm{V}_{\mathrm{m}}$ & Membrane voltage \\
\hline $\mathrm{Y} 2 \mathrm{H}$ & Yeast-Two-Hybrid (System) \\
\hline
\end{tabular}




\section{Amino acids abbreviations}

$\begin{array}{lll}\text { Alanine } & \text { Ala } & \text { A } \\ \text { Arginine } & \text { Arg } & \text { R } \\ \text { Asparagine } & \text { Asn } & \text { N } \\ \text { Aspartic acid } & \text { Asp } & \text { D } \\ \text { Cysteine } & \text { Cys } & \text { C } \\ \text { Glutamic acid } & \text { Glu } & \text { E } \\ \text { Glutamine } & \text { Gln } & \text { Q } \\ \text { Glycine } & \text { Gly } & \text { G } \\ \text { Histidine } & \text { His } & \text { H } \\ \text { Isoleucine } & \text { Ile } & \text { I } \\ \text { Leucine } & \text { Leu } & \text { L } \\ \text { Lysine } & \text { Lys } & \text { K } \\ \text { Methionine } & \text { Met } & \text { M } \\ \text { Phenylalanine } & \text { Phe } & \text { F } \\ \text { Proline } & \text { Pro } & \text { P } \\ \text { Serine } & \text { Ser } & \text { S } \\ \text { Threonine } & \text { Thr } & \text { T } \\ \text { Tryptophan } & \text { Trp } & \text { W } \\ \text { Tyrosine } & \text { Tyr } & \text { Y } \\ \text { Valine } & \text { Val } & \text { V }\end{array}$




\section{$1 \quad$ INTRODUCTION}

\subsection{Ion channels: focus on voltage gated potassium channels}

A fundamental feature of all living organisms is the separation of the aqueous internal milieu from its environment that is achieved by the cell membrane. The typical biological membrane is formed by a phospholipid bilayer that represents an enormous energy barrier to the movement of small ions across it. The cellular equilibrium, as well as diverse physiological functions, depends on the transport of ions through the cell membrane. Thus, during evolution a special class of proteins known as ion channels evolved to enable rapid ion flux through the membrane between the cell and its environment.

Ion channels are present in almost all excitable and non-excitable cells of living organisms. They can be considered as embedded in the membrane, gated pores that are permeable only to selected ions. Thus, depending on their ionic selectivity, different groups of ion channels, for example sodium or potassium channels, can be distinguished.

The flux of ions through the channel is passive and determined by electrostatic and diffusional driving forces across the membrane. Opening of the channel and thus regulation of ion flux can be provided by a variety of stimuli like ligand binding, mechanical impulses or changes in voltage gradient across the membrane. Accordingly, ion channels can be classified depending on the gating (opening or activation) mechanism into, for example, ligand-gated or voltagegated channels.

Voltage-gated ion channels are the hallmark of excitable cells, although some are expressed in many different cell types. The signal for their activation is a change in membrane potential. Therefore, one common feature of these channels is the so-called voltage sensor or paddle, a partially transmembrane domain, consisting of a set of positively charged amino acids. Upon depolarisation of the membrane the positive charges of the voltage sensor move through the electrical field, which can be detected as a transient current (gating current), and drive allosteric changes in the channels structure transforming it from the closed to the open state (Stühmer et al., 1989; Papazian et al., 1991; Jiang et al., 2003 B).

Voltage gated ion channels form a large family, whose members exhibit different ion selectivity and are therefore divided into groups, for example, calcium, potassium or sodium channels. 
According to the commonly accepted model, the fore-mentioned channels are based on the same architecture and classified as six-transmembrane one-pore voltage-gated channels. They consist of four repeats of a general motif forming $\alpha$-subunit of the channel. In sodium and calcium channels those four motif-replicas are included in a single subunit. In contrast, potassium channels are built by four separate $\alpha$-subunits which interact to form the central ion-conducting pore of the functional channel. The cluster of four subunits of the potassium channel (homo- or hetero-multimers) is topologically equivalent to the single $\alpha$-subunit in $\mathrm{Na}^{+}$or $\mathrm{Ca}^{+}$channels (Fig. 1 ).

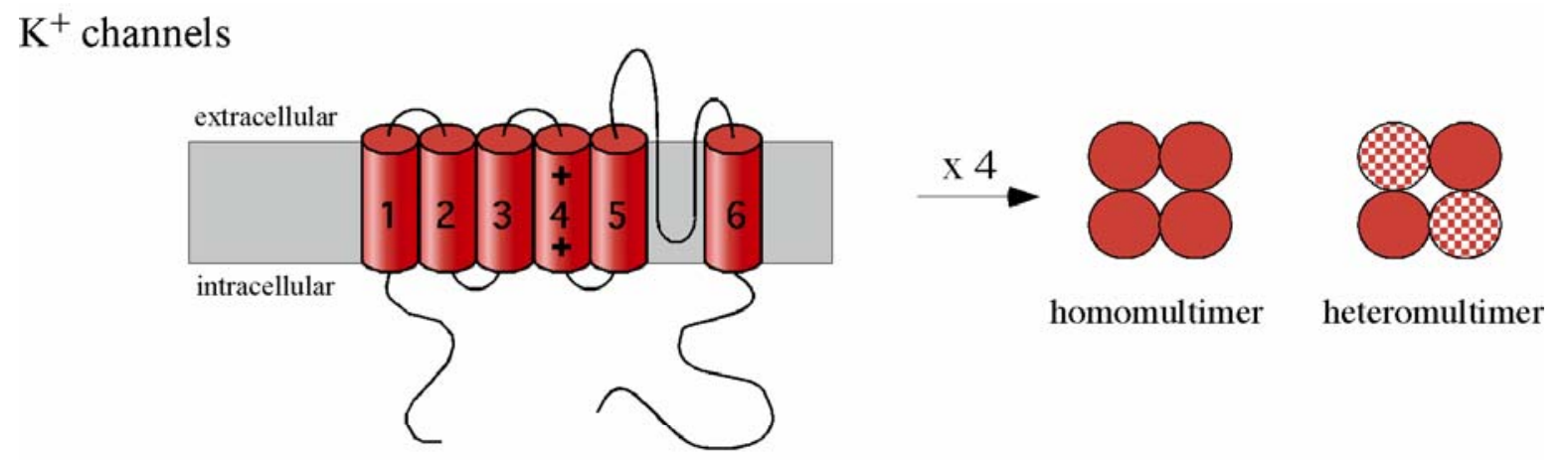

$\mathrm{Na}^{+}$and $\mathrm{Ca}^{2+}$ channels

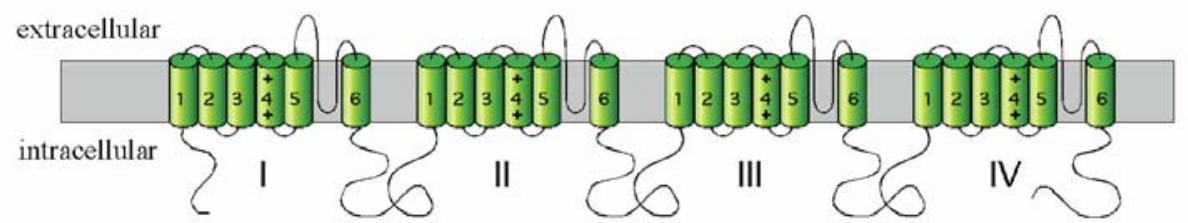

Fig. 1 Topology of voltage-gated ion channels.

A functional voltage gated potassium channel is formed by four $\alpha$-subunits, each consisting of six transmembrane domains surrounding the central pore. Four identical $\alpha$-subunits form homomultimeric, up to four different form heteromultimeric channels (only one example of heteromultimeric assembly is depicted). In sodium channels, the four subunits are linked together by intracellular loops (Modified from Terlau and Stühmer, 1998 and Choe et al., 1999).

A single subunit of voltage-gated ion channels consists of six regions (S1-S6) forming hydrophobic $\alpha$-helices spanning the membrane. An exception is the transmembrane S4 domain that has a rather amphiphilic character, since it contains the voltage-sensor motif. The helices S5 and S6 are connected by an extracellular loop, which is partially immersed in the membrane (pore loop; P) that contributes to the selectivity filter of the channel. The $\mathrm{N}$ - and C-terminal domains of these channels are facing the cytosolic side of the membrane (Guy and Conti, 1990; MacKinnon, 1991; Armstrong and Hille, 1998; Jiang et al., 2003 A). 
Due to the amphiphilic character and the large size of ion channels, it is relatively difficult to solve their three-dimensional structure by X-ray crystallography or nuclear magnetic resonance (NMR) techniques. Thus, limited information about structural arrangement of the voltage gated ion channels is available.

Structural data of the pore region of the KcsA voltage gated $\mathrm{K}^{+}$channel (consisting of only two transmembrane segments) from Streptomyces liviandis, have been obtained by X-ray crystallography (Doyle et al., 1998). The three-dimensional appearance of a sodium channel (Sato et al., 2001) and the Shaker potassium channel from Drosophila melanogaster (Sokolova et al., 2001) were resolved by electron microscopy. Consistent with the model, the Shaker channels appeared in a four-fold symmetric structure with large, transmembrane and small, cytoplasmatic domains linked through the so-called 'connectors'.

Very recently, a report presenting X-ray structure data of a voltage-dependent $\mathrm{K}+$ channel (KvAP) was published by MacKinnon’s group (Jiang et al., 2003 A; Jiang et al., 2003 B). The crystal structure of the KvAP channel is basically in agreement with the previously described model, although few additional details, mainly related to the voltage gating, prompt to revise the traditional view on the channel structure. Instead of the classical S3 helix, two helices (S3a and S3b) separated by a loop (S3 loop) were identified. S3b and S4 were found to form a predominantly hydrophobic helix-turn-helix structure, called "voltage-sensor paddle”. The four paddles are positioned nearby the intracellular surface of the membrane, perpendicular to the channel pore, when the channel is closed. Changes in membrane potential move the paddles over large distances across the membrane from inside to outside resulting in channel opening.

\subsection{Ether á go-go potassium channel}

The ether á go-go (EAG) gene, which encodes for a voltage-dependent potassium channel, was first cloned from Drosophila melanogaster (dEAG; Warmke et al., 1991) via analysis of mutations affecting membrane excitability. Mutations of the Drosophila eag locus cause spontaneous, repetitive firing in motor neurones and enhanced transmitter release at the neuromuscular junction (Wu et al., 1983). Molecular studies and sequence comparison resulted in the classification of eag into a distinct potassium channel group: the EAG family. The discovery was followed by subsequent identification of other family members, divided 
in three subgroups (Warmke and Ganetzky, 1994): eag, erg (eag-related gene) and elk (eag-like $\mathrm{K}^{+}$channels).

Like other potassium channels, Eag1 channels are suggested to form tetramers build by the assembly of four subunits, each consisting of six putative transmembrane domains (Fig. 2). The tetramerisation is mediated by the C-terminal motif, a tetramerising coiled-coil (TCC) domain (Ludwig et al., 1997; Jenke et al., 2003).

Mammalian Eag1 channels activate slowly and do not inactivate during a sustained depolarisation. Moreover, voltage dependent activation of Eag channels is strongly dependent on the holding potential. The time course of the activation is slowed down by negative prepulses, an effect similar to the Cole-Moore shift (Ludwig et al., 1994).

Eag1 seems to have diverse and broad function in cellular processes. Besides its major expression in brain, Eag1 was found in a few other tissues, mainly in highly proliferating ones, like in the myoblasts at the onset of fusion (Occhiodoro et al., 1998) and in placenta, as well as in a high number of tumour tissues and tumour cell lines (Pardo et al., 1999 and Pardo personal communication).

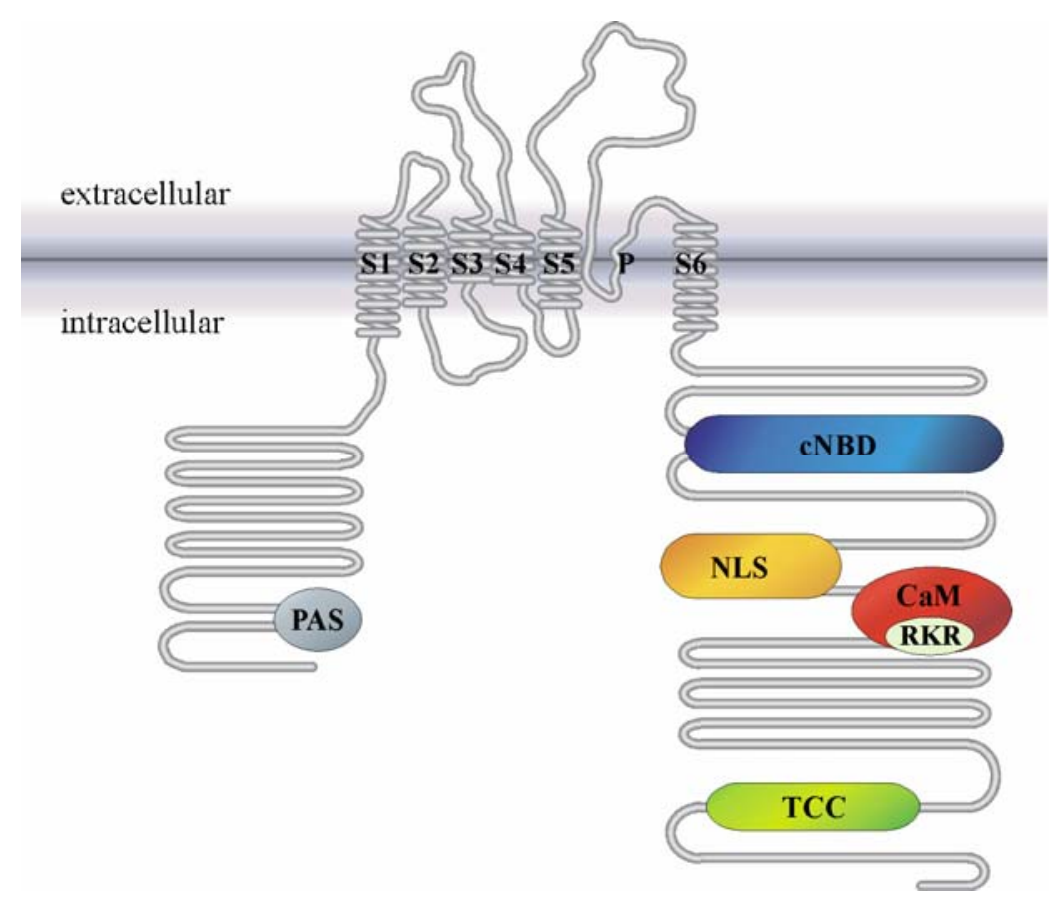

Fig. 2 Schematic depiction of the single hEag1 $\alpha$-subunit

S1-S6 represent transmembrane helices, $\mathrm{P}$ is the pore (or H5) region. The $\mathrm{N}$ - and C-terminus are believed to be located intracellularly. Characteristic domains are represented by coloured ellipses:

- PAS Per-Arnt-Sim domain

- cNBD cyclic nucleotide binding domain

- NLS nuclear localisation signal

- CaM Calmodulin binding site

- RKR endoplasmatic reticulum retention signal

- TCC tetramerising coiled-coil domain 
A correlation of Eag1 expression with the cell cycle is not only based on expression analysis. It has been described (Brüggemann et al., 1997; Pardo et al., 1998), that the electrophysiological properties of rat Eag are modified during the cell cycle, and the changes are initiated by a factor located downstream of MPF (mitosis-promoting factor). Moreover, cells expressing Eag show characteristics of malignant transformation and expression of Eag1 favours tumour progression when transfected cells are injected into SCID mice (Pardo et al., 1999).

Up to now, not much is known about regulation of Eag1 channels on the molecular level. The Eag1 sequence exhibits several characteristic features. In the N-terminal region, a PAS domain (Per-Arnt-Sim), usually used as a signal sensor domain in many signalling proteins, can be identified, although its function in Eag1 is unknown. In the C-terminal region, several domains such as the cyclic nucleotide binding domain (cNBD; Brüggemann et al., 1993), a nuclear localisation signal (NLS), or endoplasmatic reticulum retention signal (RKR) can be distinguished.

Moreover, there are three proteins described to bind to the Eag1 protein: epsin, calmodulin and KCR1. Epsin is a protein involved mainly in endocytosis. Its binding to the C-terminus of rat Eag1 alters both activation and deactivation of Eag channels, but the significance of this interaction remains unknown (Piros et al., 1999). Calmodulin (CaM) binds to the defined sequence in the C-terminus of hEag1 and inhibits the potassium currents in a calciumdependent manner. KCR1 binds to the C-terminus of rat Eag1, but its regulatory function remains unclear (Hoshi et al., 1998).

hEag1 channels form complex interactions with the cytoskeletal elements, which strongly influences channel properties (Camacho et al., 2000). Additionally, posttranslational modification sites are abundant and could contribute to the functional modulation of Eag1 channels.

\subsection{Glycosylation}

With the rationalisation that the human genome encodes only about 30 thousand different polypeptides (Venter et al., 2001), it was realised that the dynamic enzyme-catalysed chemical modifications of these polypeptides add a substantive complexity and regulational 
diversity to the cellular proteome. The addition of oligosaccharide structures to proteins and/or the subsequent modification of these structures is one of the metabolic events that significantly extend the protein divergence spectrum. Thus, glycosylation represents a level of variability that may be necessary for complex processes of higher organisms.

At the present stage of our knowledge an impressive variety of carbohydrate-peptide linkages have been described that are broadly distributed among proteins found in essentially all living organisms, ranging from eubacteria and archaebacteria (Lechner and Wieland 1989; Messner et al., 1997), plants and invertebrates (Wilson et al., 2002) to eukaryotes. Most of the soluble and membrane-bound proteins that are made in the ER, including those destined for transport to the Golgi apparatus, lysosomes, plasma membrane or extracellular space are glycoproteins.

Talking about oligosaccharide-peptide linkage it is important to distinguish between two distinct forms of the sugar attachment to the protein: glycosylation and glycation.

Glycosylation is a highly regulated process, in which the oligosaccharyl chains are coupled to proteins by a glycosydic link formed in an enzyme-catalysed reaction. Although the sugar composition and the number and size of branches in the sugar tree varies among glycans bound to a protein, among glycoproteins, and among cell types, tissues and species, glycosylation is thought to be supervised and co-ordinated by a set of different mechanisms in only poorly understood pathways.

The term glycation, in contrast, describes a complex series of spontaneous reactions in which carbohydrates non-enzymaticaly react with primary amine groups followed by rearrangement to give a stable product (Cloos and Christgau, 2002).

According to Spiro (2002) glycopeptide bonds, which are widely distributed among glycoproteins, can be arranged in five quite distinct groups including $\mathrm{N}$-linked glycosylation, O-linked glycosylation, glypiation (GPI-anchor), C-mannosylation and phosphoglycosylation.

\subsubsection{N-linked glycosylation}

$\mathrm{N}$-linked glycosylation represents the most widely distributed carbohydrate-peptide bond and is the site of attachment for a large variety of oligosaccharides (Spiro, 1973). The glycopeptide linkage is established through the cotranslational transfer of preassembled 
oligosaccharide-complexes to specific Asn residues of proteins. The tripeptide consensus sequence Asn-Xaa-Thr/Ser (where Xaa can be any amino acid besides proline) is generally required for the reaction (Kornfeld and Kornfeld, 1985; Kasturi et al., 1995). Of all glycosylation sequons shared among different proteins it has been estimated that $90 \%$ are glycosylated (Gavel and von Heijne, 1990).

Biosynthesis of N-linked glycans begins at the donor molecule that is an activated lipid carrier, a dolichylpyrophosphate. Synthesis starts with the addition of an $\mathrm{N}$-acetylglucosamine phosphate residue to the dolichyl phosphate, a reaction catalysed by $\mathrm{N}$-Acetylglucosaminyl phosphate transferase (GPT; Burda and Aebi, 1999).

Since the GPT enzyme is sensitive to the antibiotic tunicamycin (Tkacz and Lampen, 1975) this drug blocks the assembly of the lipid-linked precursor and thus acts as an inhibitor of the whole N-linked glycoprotein biosynthesis pathway. Although tunicamycin can also interfere with protein synthesis, or the glycolipid biosynthesis pathway (Guarnaccia et al., 1983; Yusuf et al., 1983), it is commonly used in studies of the N-linked glycosylation pathway.

After initiation of the glycosaccharide synthesis, the 'core-oligosaccharide' forming blocks consisting of consensus 14-sugar scaffolds are further assembled on the ER-membrane bound dolichylpyrophosphate precursor. Synthesis begins on the cytosolic side of the ER membrane, but the intermediate 7-sugar glyco-lipid complex becomes than flipped into the ER lumen where sugar chains are completed and finally transferred to an asparagine residue on a nascent polypeptide chain (Burda and Aebi, 1999). The transfer reaction is carried out by the oligosaccharyltransferase.

The N-linked carbohydrate is the only, besides GPI anchor, that is transferred to the polypeptide en block, all other sugar-amino acid linkages are formed by enzymatic transfer of an activated monosaccharide directly to the protein (Spiro, 2002).

Following the co-translational transfer of sugars to the polypeptide, oligosacharides are immediately subjected to extensive modifications as the glycoproteins mature and are moved through ER via the Golgi complex to their final destinations inside or outside the cell.

As the reticular pool of sugar modifying enzymes is relatively poor, the ER-residual core-glycans are homogenous and relatively simple (Fig. 3). The spectrum of glycoforms remains rather uniform until the glycoproteins reach the medial stacks of the Golgi apparatus where structural diversification is introduced through a series of nonuniform modifications. Assembled glycans are processed through the action of glycosyl-hydrolases and glycosyl-transferases to high-mannose, complex, or hybrid-type oligosaccharide structures. In mature glycoproteins N-linked glycan moieties are structurally diverse. This significant product micro-heterogeneity provides an excellent resource for the functional diversity in living organisms. 


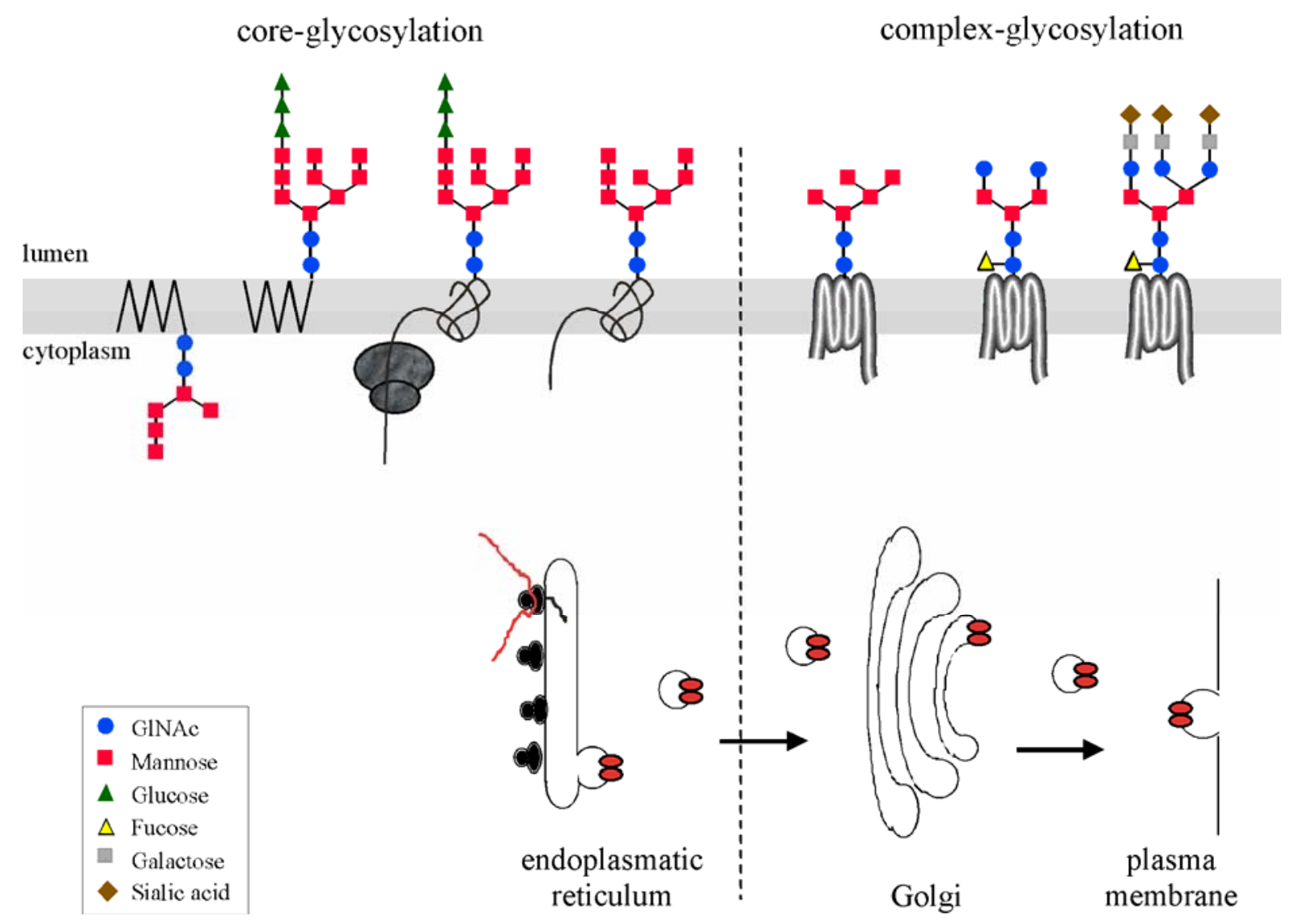

Fig. $3 \quad \mathrm{~N}$-linked glycosylation in endoplasmatic reticulum and Golgi

\subsubsection{Other glycosylation forms (O-linked glycosylation, glypiation, C-mannosylation and phosphoglycosylation)}

\section{O-linked glycosylation}

O-linked glycosylation is a complex modification that is abundantly used on nuclear and cytoplasmatic proteins by almost all known organisms. In the O-linked glycoproteins, oligosaccharides are attached to the hydroxyl group of side chains of serine, threonine or hydroxylysine, although other amino acids can be used. O-glycosylation begins posttranslationally in the Golgi apparatus with an addition of a single monosaccharide to the protein (Van den Steen et al., 1998). Currently there is no O-linked amino acid consensus sequence identified, but it is believed that the reaction is dependent on the primary, secondary, and tertiary structure of the protein. O-linked glycosylation is a reversible posttranslational modification and thus seems to have diverse functional regulatory roles for the respective proteins (Vosseller et al., 2002; Zachara and Hart 2002, Hart, 1997, Wells et al., 2001). 


\section{GPI-anchor}

In glypiation proteins are anchored to the outer leaflet of the plasma membrane through the carbohydrate-lipid complex including glycosylphosphatidylinositol (GPI). GPI-anchors are biosynthesised in the ER and are widely distributed among biologically important cell surface glycoproteins of different species (Doering et al., 1990). GPI-anchored proteins are involved in fundamental processes such as signal transduction, cell membrane processing, homeostasis, and immune responses, as well as the pathobiology of cancers and infectious diseases (Nosjean et al., 1997).

\section{C-mannosylation and phosphoglycosylation}

C-mannosylation is a recently described carbohydrate-protein bond involving the attachment of an alpha-mannosyl residue to the C-2 of Trp through a C-C bond (de Beer et al., 1995). In phosphoglycosylation the sugar is linked to serine or threonine via a phosphodiester bond (Haynes, 1998).

\subsubsection{Function of $\mathbf{N}$-linked glycosylation}

The diverse complexity of N-linked oligosaccharide structures allows them to play various biological roles. Their purposes seem to range from trivial to crucial, depending on the glycoconjugate, the oligosaccharide structure and the biological context.

The single important function of N-linked glycosylation is the promotion of proper folding of newly synthesised polypeptides in the ER (Gavel and von Heijne, 1990; Helenius, 1994). When glycosylation is inhibited, the most commonly observed effect is generation of misfolded, aggregated proteins that fail to reach a functional state and are consequently degraded (Gavel and von Heijne, 1990; Helenius, 1994; Varki, 1993).

$\mathrm{N}$-linked oligosaccharides are large hydrophobic structures, thus, by virtue of their size alone, the addition of the glycan moulds to the protein backbone can dramatically alter the structure, and consequently the function of the polypeptide architecture to which they are attached. Nlinked glycans can affect protein structure in two ways. First, since the addition of carbohydrates occurs cotranslationally to the nascent polypeptide, it can have a great impact on the protein folding process. Second, the carbohydrates can stabilise the mature protein (Imperiali and O'Connor, 1999). In general, glycoproteins are more stable than their corresponding unglycosylated counterparts, even if no major structural changes are associated with the glycosylation. 
Since cells are capable of producing a wide variety of oligosaccharyl structures, sophisticated mechanisms exist to ensure that the correct structures are added to the numerous glycoproteins that are assembled. The ER contains a high concentration of soluble and membrane bound molecular chaperones and folding enzymes controlling the folding process of proteins. Two of the chaperones, the lectins calnexin (membrane bound) and calreticulin (soluble) bind transiently to almost all newly synthesised unfolded glycoproteins, slowing down the rate and thus increasing the efficiency of their folding (Ou et al., 1993; Helenius and Aebi, 2001). In some cases inhibition of the initial glycosylation disrupts protein folding to such an extent that it induces the 'unfolded protein response', a complex process characterised by the up-regulation of several chaperones in the endoplasmatic reticulum.

Although glycosylation enhances proper folding of proteins, it is not necessary for structure formation. Other chaperones, such as $\mathrm{BiP}$ (Binding protein) can bind to unglycosylated proteins controlling their proper folding (Ellgaard et al., 1999; Molinari and Helenius, 2000).

Another function of N-linked glycosylation is protection from degradation by intracellular proteases. Simply because of their size, glycans can shield large regions of protein surfaces, providing protease protection for the attached protein. This property of the N-linked glycans is well studied in lysosomes. The lysosomal membrane contains an assortment of highly glycosylated proteins, among others LAMP and LIMP family members containing up to 20 $\mathrm{N}$-linked glycans each. These glycans have been shown to shield the underlying Lamp-1 and Lamp-2 polypeptides from their proteolytic environment in the lysosome (Kundra and Kornfeld, 1999) since their deglycosylation resulted in a rapid degradation.

Glycosylation might also serve to enhance thermal stability (Olsen et al., 1991) or to influence the solubility of proteins as well as affect the intracellular transport and localisation of the glycoprotein. In Lutropin receptor, for example, elimination of the glycosylation site causes a loss of surface expression (Ji et al., 1990). Also trafficking of ion channels is often strongly dependent on glycosylation. For example, it has been reported that $\mathrm{N}$-linked glycosylation is required for the cell surface expression of ligand-gated channels including ATP purinergic receptors: P2X1 (Rettinger et al., 2000) and P2X2 (Torres et al., 1998).

In the secretory pathway glycans have a common role in promoting protein folding, quality control, and certain sorting events at early steps. Later, Golgi enzymes perform complex sugar modifications prepare for the spectrum of novel functions that mature proteins display. Once expressed in fully mature form on the cell surface N-linked glycans can play a more global role, modulating the function of host proteins at the targeted sites. 
Sugars extending into the extracellular space are large moieties and, like in the cytosol, able to provide a 'fence' around an individual protein molecule so that interaction with other proteins attached to the membrane is hampered. The 'coating' of many plasma-membrane proteins by oligosaccharides can on the one side protect the polypeptide chain from recognition by antibodies, on the other, be involved in recognition events as such glycoconjugates can serve as ligands for receptors on other cells or pathogens. It is known that certain oligosaccharides act as highly specific receptors for a variety of viruses, bacteria, parasites and toxins, and serve as antigens for autoimmune responses (Varki, 1993). They also play role in T-cell recognition of antigen presenting cells and in formation of ‘immunological synapse’ (Rudd et al., 1999).

Since cells are covered with a dense coat of sugars it is postulated that oligosaccharides must be critical determinants of cell-cell and cell-matrix interactions, however their involvement in these processes is not well documented. In the most cases oligosaccharides seem to play an indirect role in cell-cell contacts, directing folding and transport of the functional proteins rather than having a direct function in the cellular contact.

Addition of N-linked sialic acid seems to be particularly important in cell-cell adhesion and cell migration (reviewed by Rutishauser, 1996). However, rather O- than N-linked glycosylation seems to play a straightforward role in adhesion processes.

Glycosylation can, also be required for proper function of a protein at the targeted site, such as binding to ligands or receptors. For example, mutation of one of the glycosylation sites in MCHR1 (Melanin-concentrating hormone receptor) has been shown to significantly lower the ligand binding (Saito et al., 2003).

\subsection{N-linked glycosylation and ion channels}

Ion channels are transmembrane proteins that do not differ in their origin and processing from other membrane proteins. Thus, glycosylation has to be considered as an important posttranslational modification when studying ion channel function.

With the increase of our knowledge about ion channels there is an enormous expansion of reports describing the importance of glycosylation on channel proteins. It starts with a simple fact that differential glycosylation contributes to the diversity of ion channel function. 
Glycosylation has been shown to modulate the functional properties of many ion channels. For example, inhibition of N-linked glycosylation altered the voltage dependence of channel gating of some voltage-gated ion channels, such as $\mathrm{Kv1.1}$ and $\mathrm{KvLQT} / \mathrm{minK} \mathrm{K}+\left(\mathrm{I}_{\mathrm{sK}}\right)$ (Freeman et al., 2000; Thornhill et al., 1996) or sodium channels (Zhang et al., 1999). It can also modulate open probability or $\mathrm{pH}$ sensitivity of channels. The ROMK1 inward rectifier $\mathrm{K}^{+}$ channel showed reduced open probability when $\mathrm{N}$-linked glycosylation is inhibited (Schwalbe et al., 1995), whereas expression of KvLQT/minK $\mathrm{K}^{+}$channels in nonglycosylating cells affected not only the gating properties but also the $\mathrm{pH}$ sensitivity of the channel (Freeman et al., 2000).

However, in most cases glycosylation is playing a more complex role in modulating different aspects of ion channel biogenesis, trafficking and/or function.

So, for example, N-linked glycosylation increases the stability of Shaker potassium channels but has little effect on acquisition of the native structure. It has been shown that the rapid degradation of nonglycosylated Shaker channels involves cytoplasmatic proteasomes (Khanna et al., 2001). N-linked glycosylation resulted, moreover, in faster trafficking of the Shaker protein from the endoplasmatic reticulum to the Golgi (de Souza et al., 2002), but no information about the effects of N-glycosylation on the electrophysiological properties of channels are available.

Another well-studied example is HERG, a voltage gated potassium channel. It has been reported that N-linked glycosylation is required for expression of HERG channels in the cell membrane (Petrecca et al., 1999) and plays an important role in HERG channel stability (Gong et al., 2002). Mutations affecting glycosylation of HERG channels were also found in the patients with LQT syndrome (a HERG associated cardiac disorder) demonstrating a physiological significance of the glycosylation process (Satler et al., 1998; Zhou, 1998 B). Moreover, a HERG mutant, lacking one of two functional glycosylation sites (N629), failed to generate HERG currents when expressed in mammalian cells (Gong et al., 2002).

Those and many other examples suggest that $\mathrm{N}$-linked glycosylation is playing an important role in proper functioning of many membrane proteins including ion channels. The diversity of glycans attached to ion channels may regulate a variety of cellular activities, beginning with modulation of the protein spectrum expressed on the plasma membrane, up to the regulation of cell responses to extrinsic stimuli. 


\subsection{Aim of the study}

The primary goal of this study was the analysis of Eag1 on the protein level, its expression pattern and eventual posttranslational modifications. Since we found that rat Eag1 undergoes $\mathrm{N}$-linked glycosylation in brain, a common and powerful modification of plasma membrane proteins, the following questions evaluated:

- does the human Eag1 also undergo N-linked glycosylation

- what is the function of the N-linked glycosylation of hEag1 channels

To study this we decided to use two expression models: CHO cells for the biochemical characterisation and Xenopus laevis oocytes for the functional analysis. We used both site directed mutagenesis and internal block of the glycosylation pathway, as well as different biochemical and electrophysiological methods, in order to investigate the $\mathrm{N}$-linked glycosylation of hEag1 channels.

The aims of the study were:

- to identify sites responsible for oligosaccharide attachment

- to propose a model of N-linked glycosylation of hEag1 channels

- to study the effects of attached oligosaccharide(s) on the intracellular localisation of hEag1

- to investigate functional effects of N-linked glycosylation of hEag1 channels. 


\section{MATERIALS AND METHODS}

\section{$2.1 \quad$ Materials}

\subsubsection{Equipment}

Centrifuges

Confocal microscope

Developing machine

Fluorescence microscope (Axioskop)

Homogeniser

manipulator (stepper motor driven LN SM-1)

Mini-PROTEAN II gel system

patch-clamp amplifier (EPC-9)

Photometer

pipette puller (HEKA PIP5)

TRIO-Thermoblock ${ }^{\mathrm{TM}}$ (PCR)

Turbo TEC-10CD amplifier
XL-90, Beckman

Optima TLX 120, Beckman

5415 C, Eppendorf (Table-centrifuge)

5402, Eppendorf (Cooling-centrifuge)

LSM 510 with Axiovert 200, Zeiss

Curix 60, AGFA

Zeiss

Schütt Labortechnik

Luigs \& Neumann

BIO-RAD Laboratories

HEKA Electronic

Eppendorf

HEKA Electronic

Biometra

NPI-Elektronik

\subsubsection{Chemicals, reagents and material}

Acrylamide/Bisacrylamide Solution $(30 \% ; 37,5: 1)$

Agar

Agarose

Ammonium peroxydisulphate

Ampuwa ${ }^{\mathrm{TM}}$
BIO-RAD Laboratories

Gibco/BRL

Gibco/BRL

Fluka

Fresenius AG 
Bromophenolblue

Chloroform

Collagenase

Commasie R250 $\mathrm{TM}$

Concanavalin A-Agarose

Dextran sulfate

Deoxycholic Acid, Sodium Salt

Diethyl pyrocarbonate (DEPC)

Dimethylsulfoxide (DMSO)

Dithiothreitol (DTT)

dNTPs (100 mM)

Ethylenediamine tetraacetic acid (EDTA)

Ethidium bromide

Films (X-OMAT Blue XB1)

Filter $0,22 \mu \mathrm{m}$

Formaldehyde

Formamide

Glass capillaries

Hoechst 33342

Hybond ECL ${ }^{\mathrm{TM}}$ Nitrocelulose Membrane

Isoamyl alcohol

B-Mercaptoethanol

Paraformaldehyde

Phenol

Photographic Fixer

Photographic Developer

Protein A-Agarose

Protein G/A-Agarose

Sodium Dodecyl Sulfate (SDS)

$\mathrm{N}^{\prime} \mathrm{N}^{\prime} \mathrm{N}^{\prime} \mathrm{N}$ Tetramethylethyldiamine (TEMED)

Tris-Base
Sigma

Sigma

Worthington Biochemical corporation

Biomol

Calbiochem

Amersham

Sigma

Sigma

Sigma

Sigma

Boehringer

Sigma

Fluka

Kodak

Milipore

Fluka

Fluka

Hilgenberg

Hoechst

Amersham Biosciences

Fluka

Sigma

Fluka

Gibco/BRL

Agfa

Agfa

Oncogene Research Products

Oncogene Research Products

Fluka

Sigma

Sigma 
Tween 20

(Polyoxyethylenesorbitan monolaurate)

Triton X-100

Whatman ${ }^{\mathrm{TM}}$ filter paper

$\mathrm{X}-\alpha-\mathrm{Gal}$

$\mathrm{X}-\beta-\mathrm{Gal}$

X-Omat ${ }^{\mathrm{TM}}$ Blue Films
Sigma

Sigma

Schleicher \& Schüll

BD Biosciences Clontech

Biomol

Kodak

Chemicals, which are not listed above, were purchased form Merck, Darmstadt.

\subsubsection{Antibiotics}

Ampicillin

Cefuroxime/Zinacef750

Geneticin $^{\mathrm{TM}}$ (G418 sulphate)

Kanamycin

Penicillin

Streptomycin

Zeocin
Roche

Aventis

Calbiochem

Roche

Gibco

Gibco

Calbiochem

\subsubsection{Enzymes, inhibitors, substrates}

Accutase $^{\mathrm{TM}}$

Bovine Serum Albumine (BSA)

Complete Protease Inhibitor Cocktail Tablets (EDTA free)

DNase (RQ1) RNase free

Endoglycosidase $\mathrm{H}$ (Endo $\mathrm{H}$ )

Leupeptin

Pepstatin A

Peptide N-Glycosidase F (PNGase F)
PAA

Sigma

Roche

Promega

Roche

Sigma

Sigma

Roche 
Phenylmethylsulfonyl fluoride (PMSF)

Proteinase K

Phosphatase, alkaline

Poly-L-Lysin

RNasin $^{\mathrm{TM}}$ (RNase inhibitor)

$\mathrm{T}_{4}$-DNA-Ligase

$\mathrm{T}_{4}$-RNA-Polymerase

TaqPlus $^{\mathrm{TM}}$ Precision DNA Polymerase

Tunicamycin
Serva

Roche

Promega

Sigma

Promega

Promega

Promega

Stratagene

Sigma

\title{
2.1.5 Standards
}

\author{
RNA Ladder \\ Invitrogen \\ $1 \mathrm{~kb}$ DNA Ladder \\ Gibco/BRL \\ 100 bp DNA Ladder \\ Gibco/BRL \\ Precision Protein Standard ${ }^{\mathrm{TM}}$ Broad Range \\ BIO-RAD Laboratories
}

(Prestained)

\subsubsection{Kits and ready-to-use reagents}

\begin{tabular}{|c|c|}
\hline BCA $^{\mathrm{TM}}$ Protein-Assay & Pierce (Perbio Science) \\
\hline DAC- $-30^{\mathrm{TM}}$ & Eurogentec \\
\hline $\mathrm{ECL}^{\mathrm{TM}}$ & Amersham Biosciences \\
\hline $\begin{array}{l}\text { EndoFree }^{\mathrm{TM}} \\
\quad \text { Endotoxin-free Plasmid Maxi Kit }\end{array}$ & Qiagen \\
\hline Fugene ${ }^{\mathrm{TM}}$ & Roche \\
\hline $\begin{array}{l}\text { MATCHMAKERTM GAL4 } \\
\text { Two-Hybrid System } 3\end{array}$ & Clontech \\
\hline Mycoplasma Plus ${ }^{\mathrm{TM}}$ PCR Primer Set & Stratagene \\
\hline NucleoSpin ${ }^{\mathrm{TM}}$ Extract 2 in 1 & Macherey-Nagel \\
\hline NucleoSpin ${ }^{\mathrm{TM}}$ Plasmid & Macherey-Nagel \\
\hline
\end{tabular}




$\begin{array}{ll}\text { Plasmid Maxi Kit } & \text { Qiagen } \\ \text { ProLong Antifade Kit } & \text { Molecular Probes } \\ \begin{array}{l}\text { ProSTAR }{ }^{\mathrm{TM}} \text { HF Single-Tube } \\ \text { RT-PCR System }\end{array} & \text { Stratagene } \\ \text { Qiaex II TM } & \text { Qiagen } \\ \text { QuickChange }^{\mathrm{TM}} \text { Site-Directed Mutagenesis Kit } & \text { Qiagen } \\ \text { RiboGreen }^{\mathrm{TM}} \text { RNA Quantitation Kit } & \text { Molecular Probes } \\ \text { StrataPrep }^{\mathrm{TM}} \text { Total RNA Microprep Kit } & \text { Stratagene } \\ \text { Total RNA Isolation Reagent }^{\mathrm{TM}} \text { Transformation System 2 } & \text { BD Biosciences Clontech } \\ \text { YEASTMAKER }^{\text {YMOL }}\end{array}$

\subsubsection{Plasmids}

pSGEM Prof. Michael Hollman, Bochum University

A vector based on pGEM-3Z vector containing 3' and 5' UTR from a Xenopus laevis $\beta$-globin gene flanking a polylinker derived from the MCS of pBluescript Vector. Contains a cassette of four restriction sites upstream of the SP6 promoter that can be used for sense template linearisation.

pTracer Invitrogen

Mammalian expression vector. Encode GFP-Zeocin fusion protein. CMV promoter.

pCDNA 4 myc/His $\mathrm{B}+\quad$ Invitrogen

Mammalian expression vector. Contains human CMV promoter, as well as c-myc epitope and polyhistidine Tag (C-Terminus) and Zeocin resistance gene.

Generates fusion of GAL4 activation domain, HA epitope tag and a protein of interest. Harbours Ampicillin resistance and Leu2 nutritional genes for selection in bacteria and yeast, respectively. 
pGBKT7 BD Biosciences Clontech

Express a fusion protein of the GAL4 DNA-binding domain, c-myc epitope tag and bait protein. Harbours Kanamycin resistance and Trp nutritional genes for selection in bacteria and yeast, respectively.

\subsubsection{Synthetic oligonucleotide primers}

Synthetic oligonucleotide primers were used for PCR or RT-PCR reactions, sequencing of DNA constructs or introduction of mutations into the plasmid DNA. Primers were synthesised by Metabion (Planegg-Martinsried) in HPLC purity grade. All oligonucleotides are depicted in 5' to 3' orientation.

\subsubsection{Sequencing and PCR primers}

\begin{tabular}{llll}
\hline Name & Sequence & Direction & Template \\
\hline 853 & CCAAGACAATCCGCAACAACA & forward & hEag1 \\
854 & GTGCAAAAAGGCGAGAATGTC & forward & hEag1 \\
855 & CGCATGAACTACCTGAAGACG & forward & hEag1 \\
856 & GCCCAGTCCTGTGCCAATGTT & forward & hEag1 \\
857 & CAAGTGTCCAAGGCTGAGTCG & forward & hEag1 \\
858 & CGTCACCACCACCCCTACCAC & forward & hEag1 \\
859 & CTGAGCATTCCCCAACACAAA & reverse & hEag1 \\
860 & GCACAATGTCCACCAAAAAGA & reverse & hEag1 \\
861 & TCTGTGGATGGGGCGATGTTC & reverse & hEag1 \\
862 & AGGGTGGTCCGGGGGCAAGAT & reverse & hEag1 \\
863 & GTGACGGCAGGGTTGGAGGTA & reverse & hEag1 \\
CMV for & CGCAAATGGGCGGTAGGCGTG & forward & pTracer \\
T7 & TAATACGACTCACTATAGGG & forward & pCDNA4 \\
GAL4 AD for & ATACCACTACAATGGAT & forward & pACT2 \\
5'AD LD for & CTATTCGATGATGAAGATACCCCACCA & forward & pACT2 \\
Y2H AD rev & GTTGAAGTGAACTTGCG & reverse & pACT2 \\
3'AD LD rev & GTGAACTTGCGGGGTTTTTCAGTATCTACGA & reverse & pACT2 \\
\hline
\end{tabular}




\subsubsection{Oligonucleotides introducing mutation}

Introduced mutation is shown in bold and red.

\begin{tabular}{llll}
\hline Name & Sequence & Direction & Mutation \\
\hline 1939 & GTTCGGCGGTCCCAGGATACTAATTTTG & forward & hEag1 N26Q \\
1940 & CAAAATTAGTATCCTGGGACCGCCGAAC & reverse & hEag1 N26Q \\
1941 & CAGCCATCTTGGTCCCTTATCAGGTCTCC & forward & hEag1 N238Q \\
1942 & GGAGACCTGATAAGGGACCAAGATGGCTG & reverse & hEag1 N238Q \\
1944 & GACACCAAGACAATCCGCCAGAACAGC & forward & hEag1 N388Q \\
1945 & GCTGTTCTGGCGGATTGTCTTGGTGTC & reverse & hEag1 N388Q \\
1946 & CAGTTTCAGGGGTCTGGCTCAGGGAAG & forward & hEag1 N406Q \\
1947 & CTTCCCTGAGCCAGACCCCTGAAACTG & reverse & hEag1 N406Q \\
1948 & CATCTTCGGGCAGGTGACGACTATTTTCC & forward & hEag1 N470Q \\
1949 & GGAAAATAGTCGTCACCTGCCCGAAGATG & reverse & hEag1 N470Q \\
1950 & GCATGCCTCCGCCCAGCACAGCCTCG & forward & hEag1 N752Q \\
1951 & CGAGGCTGTGCTGGGCGGAGGCATGC & reverse & hEag1 N752Q \\
XhoSTOP & GACCCTCGAGCTGGCTCCAAAAATGTCTCTCTCTG & reverse & XhoI restriction \\
& ATTCTGGGG & & site in hEag1 \\
& & STOP codon \\
\hline
\end{tabular}

\subsubsection{Antibodies}

Primary and secondary antibodies described below were used either for Western blot, immunoprecipitation or immunocytochemistry experiments.

\subsubsection{Primary antibodies}

$\underline{2413 x}$

Dr. J. Pineda Antikörper servis

Rabbit polyclonal antibody directed against rat Eag1 antigen (C-terminus). Recognises rat and human Eag1 and Eag2. Used for western blotting, IP and immunofluorescence. 
$\underline{\mathrm{mAb}-33} \quad$ Biogenes

Mouse monoclonal antibody directed against C-terminus of hEag1. Does not cross-react with Eag2. Used for IP and immunofluorescence.

$\underline{\mathrm{mAb}-62} \quad$ Biogenes

Mouse monoclonal antibody directed against the pore region of hEag1. Does not crossreact with Eag2. Used for IP and immunofluorescence.

$\underline{\text { anti-AMPA }} \quad$ Dr. Maria Rubio, MPI Göttingen

Rabbit polyclonal antibody. Used for immunoprecipitation.

$\underline{\text { anti-calreticulin }}$

Dr. Majoul Irina, MPI Göttingen

Rabbit polyclonal antibody. Marker of endoplasmatic reticulum. Used for immunofluorescence.

anti-Cathepsin D Dr. Stefan Höning, Göttingen University

Rabbit polyclonal antibody. Marker of lysosomes. Used for immunofluorescence.

$\underline{\text { anti-GM-130 }} \quad$ Transduction Laboratories

Mouse monoclonal antibody directed against GM-130. Recognises human, dog, mouse and rat GM-130. Used for immunofluorescence and western-blotting.

$\underline{\operatorname{anti}-\text { Lamin A/C }} \quad$ Santa Cruz Biotechnology

Goat polyclonal antibody. Used for precipitation

anti-Limp2 $\quad$ Dr. Stefan Höning, Göttingen University

Rabbit polyclonal antibody. Marker of lysosomes. Used for immunofluorescence.

$\underline{\text { anti-p53 }}$ Dr. Majoul Irina, MPI Göttingen

(protein 53) Rabbit polyclonal antibody. Marker of endoplasmatic reticulum. Used for immunofluorescence.

$\underline{\text { anti-Rab5 }}$

Dr. Majoul Irina, MPI Göttingen

Rabbit polyclonal antibody. Marker of early endosomes. Used for immunofluorescence. 
$\underline{\text { anti-Rab7 }}$

Dr. Majoul Irina, MPI Göttingen

Rabbit polyclonal antibody. Marker of late endosomes. Used for immunofluorescence.

$\underline{\text { anti-TRAP } \alpha}$

upstate biotechnology

Mouse monoclonal antibody directed against human TRAP $\alpha$. Recognises also TRAP $\alpha$ from $\mathrm{CHO}$ cells. Used for immunofluorescence and western blotting.

\subsubsection{Secondary antibodies}

\begin{tabular}{ll}
\hline Name & \\
\hline anti-rabbit IgG HRP conjugate & Oncogene Research Products \\
anti-mouse IgG HRP conjugate & Ammersham \\
anti-mouse IgG Alexa Fluor 488 and 546 & Molecular Probes \\
anti-rabbit IgG Alexa 488 Fluor and 546 & Molecular Probes \\
anti-goat IgG Alexa Fluor 488 and 546 & Molecular Probes \\
\hline
\end{tabular}

\subsubsection{Organisms and growth media}

\subsubsection{Bacterial strain genotypes}

Competent cells from E. coli strains XL1 Blue (Young and Davis, 1983) or ready-to-use competent E. coli cells DH5 $\alpha$ (Hanahan, 1983) were used for plasmid transformation.

\begin{tabular}{|c|c|c|}
\hline Strain & Genotype & \\
\hline \multirow[t]{2}{*}{$\underline{\text { XL1 Blue }}$} & recA1 endA1 gyrA96 thi-1 hsdR17 supE44 relA1 & Stratagene \\
\hline & lac $\left[\mathrm{F}^{\prime}\right.$ proAB lacI q Z $\bullet \mathrm{M} 15$ Tn10 (Tet $\left.\left.\mathrm{r}\right)\right]$ & \\
\hline \multirow{3}{*}{$\begin{array}{l}\text { DH5a } \\
\text { Competent cells }\end{array}$} & $\mathrm{F}^{\prime} / \mathrm{endA} 1, \mathrm{hsdR} 17(\mathrm{rK}-, \mathrm{mK}+$ ), supE44, thi-1, & Invitrogen \\
\hline & recA1, gyrA(Na1 r ),relA1, D(lacZYA-argF) & \\
\hline & U169, (j801acZDM15) & \\
\hline
\end{tabular}




\subsubsection{Bacterial media}

Bacterial LB (Lura Broth) Medium was obtained as a ready to use powder mix (Gibco,BRL) and dissolved in the appropriate amount of $\mathrm{H}_{2} \mathrm{O}$. Solid media were produced adding agar at a final concentration of $1.5 \%(\mathrm{w} / \mathrm{v})$. Agar plates were poured, dried overnight at room temperature and stored at $4^{\circ} \mathrm{C}$.

After autoclavation LB or LB-agar media were supplemented with sterile antibiotics:

$\begin{array}{ll}\text { Ampicilin } & 100 \mu \mathrm{g} / \mathrm{ml} \\ \text { Kanamycin } & 50 \mu \mathrm{g} / \mathrm{ml}\end{array}$

$\underline{\mathrm{SOB} \text { medium }(500 \mathrm{ml})}$

\begin{tabular}{|c|c|c|}
\hline 2 & $\% \mathrm{v} / \mathrm{W}$ & Bacto-Trypton \\
\hline 0,5 & $\% \mathrm{v} / \mathrm{W}$ & Yeast-Extract \\
\hline 10 & $\mathrm{mM}$ & $\mathrm{NaCl}$ \\
\hline 5 & $\mathrm{mM}$ & $\mathrm{KCl}$ \\
\hline 10 & $\mathrm{mM}$ & $\mathrm{MgCl}_{2}$ \\
\hline 10 & $\mathrm{mM}$ & $\mathrm{pH} 6,8-7,0$ \\
\hline
\end{tabular}

Before addition of magnesium salts, media were sterilised by autoclavation. $\mathrm{Mg}^{2+}$ salts were prepared separately, sterile filtered and added to the autoclaved medium.

$\underline{\text { TB medium }(300 \mathrm{ml})}$

$10 \mathrm{mM}$ MOPS (free acid)

$15 \mathrm{mM} \mathrm{CaCl} 2 \times 2 \mathrm{H}_{2} \mathrm{O}$

$230 \mathrm{mM} \mathrm{KCl}$

$55 \mathrm{mM} \mathrm{MnCl}_{2} \times 4 \mathrm{H}_{2} \mathrm{O}$

All chemicals besides $\mathrm{MnCl}_{2}$ were resuspended in $80 \%$ end volume of $\mathrm{H}_{2} \mathrm{O}$ and $\mathrm{pH}$ was set to 6,7 (with $\mathrm{KOH}$ ). Afterwards, the appropriate amount of $\mathrm{MnCl}_{2}$ was added, the volume was adjusted and the solution was sterile filtered. 


\subsubsection{Yeast strain genotypes}

\begin{tabular}{|c|c|c|c|c|}
\hline Strain & Genotype & $\begin{array}{l}\text { Reporter } \\
\text { gene }\end{array}$ & $\begin{array}{l}\text { Transformation } \\
\text { marker }\end{array}$ & Reference \\
\hline$\underline{Y 187}$ & 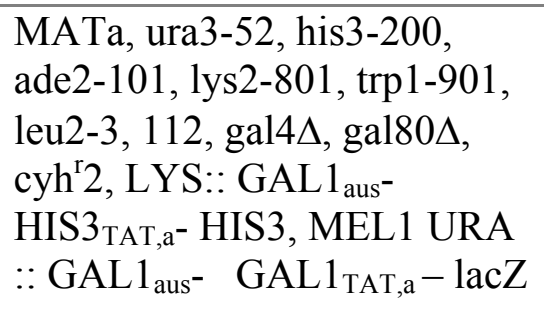 & $\begin{array}{l}\text { lacZ, } \\
\text { MEL1 }\end{array}$ & $\operatorname{trp} 1$, leu2 & $\begin{array}{l}\text { Harper et al., } \\
1993\end{array}$ \\
\hline AH109 & 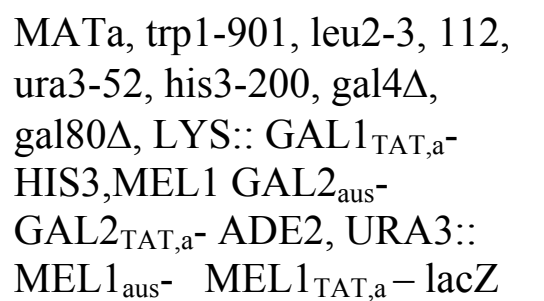 & $\begin{array}{l}\text { HIS3, } \\
\text { ADE2, } \\
\text { lacZ, } \\
\text { MEL1 }\end{array}$ & $\operatorname{trp} 1$, leu2 & $\begin{array}{l}\text { James et al., } \\
\text { 1996; }\end{array}$ \\
\hline
\end{tabular}

\subsubsection{Yeast media}

All yeast media were obtained from Clontech as ready-to-use powder mix and dissolved in the appropriate amount of $\mathrm{H}_{2} \mathrm{O}$. If necessary, additional supplements were added.

The following media were used:

$\begin{array}{ll}\underline{\text { YPD }} & \text { complete culture medium } \\ \text { YPDA } & \text { YPD medium supplemented after autoclavation and cooling down to } \\ & 50^{\circ} \mathrm{C} \text { with sterile filtered adenine to a final concentration of } 0,003 \% . \\ & \text { minimal culture medium; for auxotroph selection SD medium was } \\ & \text { supplemented with nutrient supplements lacking the appropriate } \\ & \text { component(s): } \\ & \text {-Leu, } \\ & \text {-Trp, } \\ & \text {-Leu/-Trp (LT) }\end{array}$

Additionally, the following agar plates were poured, dried for at least $4 \mathrm{~h}$ at $30^{\circ} \mathrm{C}$ or overnight at room temperature and stored at $4^{\circ} \mathrm{C}$ before use: 
YPDA-agar YPD-agar medium; autoclaved and after cooling down to $50^{\circ} \mathrm{C}$ supplemented with $7,5 \mathrm{ml}$ of $0,2 \%$ adenine (final concentration $0,003 \%$ ).

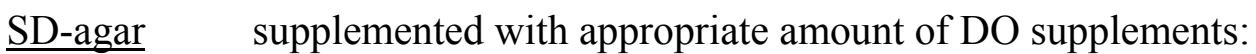

-Leu,

-Trp,

-Leu/-Trp (LT),

-His/-Leu/-Trp (LTH),

-Ade/-His/-Leu/-Trp (LTHA)

$\underline{\text { Adenin stock solution }}$

$0,2 \% \mathrm{v} / \mathrm{w}$ in sterile $\mathrm{H}_{2} \mathrm{O}$. Sterilised by filtration. Aliquots stored at $-20^{\circ} \mathrm{C}$

$\underline{\text { X- } \alpha \text {-Gal stock solution }} 20 \mathrm{mg} / \mathrm{ml}$ in dimethyl formamide

$\underline{3 \text { Aminotiazol(3AT) }} \quad 10 \mathrm{mM}$ final concentration

\subsubsection{Eucaryotic cell lines}

CHO-K1 ATCC

Chinese Hamster Ovary: CHO-K1 cell line was derived as a subclone from the parental $\mathrm{CHO}$ cell line initiated from a biopsy of an ovary of an adult Chinese hamster.

Lec1

ATCC

Mutant clonal derivate of CHO clone Pro-5. Lec1 lacks the glycosyltransferase GlcNAc-T1 so that N-linked carbohydrates are blocked at the Man5GlcNAc2Asn intermediate.

\subsubsection{Eucaryotic cell culture media}

\begin{tabular}{llll}
\hline Cell line & Medium & Additives & \\
\hline CHO-K1 & Ham's F 12 Medium & $10 \%$ FCS & Gibco \\
Lec1 & MEM Alpha Medium & $10 \%$ FCS & Gibco \\
\hline
\end{tabular}




\subsubsection{Animals}

Adult male Wistar-rats (Rattus norvegicus) were used. Rats were killed by decapitation under $\mathrm{CO}_{2}$ anaesthesia and organs were dissected immediately.

Oocytes from adult Xenopus laevis, South African clawed frogs, were used for electrophysiological measurements. Following anaesthesia, a small incision was made, which was closed immediately after removal of oocytes. Frogs were returned to the water directly after surgery.

\subsubsection{Sterilisation of solutions and equipment}

Heat insensitive solutions were sterilised at $121^{\circ} \mathrm{C}, 105 \mathrm{~Pa}$ for 60 min (Yeast culture media for $15 \mathrm{~min}$ ) in an autoclave (3850EL; Systec GmBH, Labor Systemtechnik, Wettenberg). Heat sensitive solutions were filtered through a disposable sterile filter $(0.2$ to $0.45 \mu \mathrm{m}$ pore size). Plastic ware was obtained sterilised. RNase-free solutions were prepared by overnight incubation with $0,1 \%$ DEPC and autoclaved as described. RNase-free glassware was baked overnight in an oven at $220^{\circ} \mathrm{C}$.

\section{$2.2 \quad$ Methods}

\subsubsection{Bacteria and yeast culture techniques}

Bacterial strains were grown in the described media (see 2.1.10.2) at $37^{\circ} \mathrm{C}$. For short-time storage, agar-plates with bacteria were sealed with Parafilm and stored at $4{ }^{\circ} \mathrm{C}$. For long time storage plasmid DNA was isolated and retransformation was performed.

Yeast strains were grown in the described media (see 2.1.10.4) at $30^{\circ} \mathrm{C}$. For short time storage, streaked yeast plates were sealed with Parafilm and stored at $4^{\circ} \mathrm{C}$. If necessary, yeast 
colonies were re-streaked onto fresh plates every four weeks. For long time storage, $0,5 \mathrm{ml}$ of overnight cultures were mixed with equal volume of $50 \%$ glycerol, rapidly frozen in liquid nitrogen and then stored at $-80^{\circ} \mathrm{C}$.

\subsubsection{Preparation of Epicurian Coli XL1-Blue competent bacteria}

Competent bacteria were produced by modification of cell wall, which facilitates DNA uptake. For this, $250 \mathrm{ml}$ of SOB medium was inoculated with several (3-5) colonies of E.coli strain XL1-Blue (from stock plate) and a culture was grown at $18^{\circ} \mathrm{C}, 200 \mathrm{rpm}$ until $\mathrm{OD}_{600}=0,45-0,6$ (about $48 \mathrm{~h}$ ). Bacteria were centrifuged for $10 \mathrm{~min}$ at $4^{\circ} \mathrm{C}$ at $2500 \mathrm{x} \mathrm{g}$ (swinging bucket). Pellet was resuspended in $80 \mathrm{ml}$ of sterile, ice-cold TB medium and incubated on ice for $10 \mathrm{~min}$.

The suspension was thereafter centrifuged for $10 \mathrm{~min}$ at $4^{\circ} \mathrm{C}, 2500 \mathrm{xg}$. The pellet of bacteria was resuspended in $20 \mathrm{ml}$ of sterile, ice-cold TB medium and 1,4 $\mathrm{ml}$ of DMSO was slowly added to the tube (7\% DMSO end-concentration). The mixture was incubated on ice for $10 \mathrm{~min}$, dispensed into $200 \mu \mathrm{l}$ aliquots, shock frozen in liquid nitrogen and stored at $-80^{\circ} \mathrm{C}$. An aliquot was tested for transformation efficiency (approximately $10^{8}$ transformants per $1 \mu \mathrm{g}$ of control plasmid DNA).

\subsubsection{Transformation of competent bacteria}

Transformation of competent E.Coli XL1-Blue bacteria was carried out by the heat shock procedure with an efficiency of $10^{6}-10^{8}$ transformants for $1 \mu \mathrm{g}$ of DNA. A $100 \mu 1$ aliquot of competent cells was allowed to thaw on ice and was gently mixed with 1-10 ng of DNA or $5-10 \mu 1$ of a ligation mix. After incubation for $20 \mathrm{~min}$ on ice, bacteria were heat-shocked for $1 \mathrm{~min}$ at $42^{\circ} \mathrm{C}$ and chilled for $3 \mathrm{~min}$ on ice. Following, $500 \mu \mathrm{l}$ of LB medium were added and bacteria were allowed to grow for $1 \mathrm{~h}$ at $37^{\circ} \mathrm{C}$ with gentle agitation. Finally, cells were plated onto LB-agar plates containing appropriate antibiotics and grown overnight at $37^{\circ} \mathrm{C}$. 


\subsubsection{Nucleic acid preparation, modification, amplification and analysis}

\subsubsection{Isolation of plasmid DNA}

Plasmid DNA, depending on its final purpose, was isolated either in small- or large-scale preparations. The isolated DNA was further used for transfection, sequencing, restriction analysis, PCR or subcloning.

\subsection{Small-scale preparation of plasmid DNA (Mini prep)}

MACHEREY-NAGEL NucleoSpin ${ }^{\mathrm{TM}}$ Plasmid Kit was used to purify small amounts of plasmid DNA. For this, $5 \mathrm{ml}$ of LB medium containing the appropriate antibiotics were inoculated with a single E.Coli colony and incubated for $10-16 \mathrm{~h}$ at $37^{\circ} \mathrm{C}, 225 \mathrm{rpm}$. Bacterial cultures were centrifuged $(6200 \mathrm{x} \mathrm{g}, 5 \mathrm{~min})$, and the pellet was treated according to the manufacturer's protocol. DNA was eluted with $50 \mu \mathrm{l}$ of $\mathrm{H}_{2} \mathrm{O}$

\subsection{Large-scale preparation of plasmid DNA (Maxi prep)}

For the preparation of large-scale bacterial cultures, $5 \mathrm{ml}$ of LB medium containing appropriate antibiotics were inoculated with a single colony and incubated for $6-8 \mathrm{hrs}$ at $37^{\circ} \mathrm{C}$, $225 \mathrm{rpm}$. Thereafter, $100 \mathrm{ml}$ of LB medium with appropriate antibiotic was inoculated with $0,2 \mathrm{ml}$ of this culture and incubated overnight at $37^{\circ} \mathrm{C}$ with vigorous shaking $(225 \mathrm{rpm})$. The plasmid DNA was isolated with the Plasmid Maxi Kit (Qiagen) according to the user's manual. To obtain endotoxin-free DNA, necessary for transfection of eucaryotic cells, EndoFree $^{\text {TM }}$ Plasmid Maxi Kit (Qiagen) was used. DNA was finally dissolved in 100-500 $\mu 1$ of sterile $\mathrm{H}_{2} \mathrm{O}$.

\subsubsection{Isolation of DNA fragments from agarose gels}

For the isolation of DNA fragments from agarose gels the QIAEX-IITM kit (Qiagen) or NucleoSpin ${ }^{\mathrm{TM}}$ Extract kit (Macherey-Nagel) were applied. After separation of DNA on $0,7-1,0 \%$ agarose gels, the desired DNA band was excised with a razor blade, weighed and treated as described in the user's manual. Isolated DNA fragments were tested on agarose gels and further used for subcloning. 


\subsubsection{Enzymatic modification of DNA}

\subsection{Treatment of the DNA with restriction enzymes}

Restriction enzyme digestion was carried out on the double-stranded DNA according to standard protocols (Sambrook et al., 1989). Reactions were usually incubated for 1-3 hours, in some cases also over night, to ensure complete digestion. The optimal temperature for enzyme activity was typically $37^{\circ} \mathrm{C}$ with exception of the SfiI enzyme that was incubated at $50^{\circ} \mathrm{C}$.

\subsection{Dephosphorylation of 5' ends of DNA}

To prevent the recircularisation of plasmids without insertion of foreign DNA, alkaline phosphatase treatment was performed. Alkaline phosphatase catalyses the hydrolysis of 5'-phosphate residues from DNA.

The following was mixed in a total volume of $50 \mu 1$ :

1-5 $\mu \mathrm{g}$ digested vector DNA,

$5 \mu 1 \quad 10 \times$ reaction buffer,

$1 \quad \mu \mathrm{l}$ alkaline phosphatase (1 U)

The reaction was carried out in two steps: 15 and $45 \mathrm{~min}$ (in case of 3' overhang or blunt ends produced by the specific restriction enzyme) or twice $30 \mathrm{~min}$ (5' overhangs). In both cases, an additional $1 \mu 1$ of the enzyme was added after the first incubation step. Dephosphorylated DNA was purified by phenol/ chloroform extraction and ethanol precipitation, and tested on agarose gels.

\subsection{Ligation of DNA fragments}

The ligation of DNA inserts into vector DNA was carried out in the following reaction mix, in a total volume of $10 \mu 1$ :

$$
\begin{array}{ccl}
25-50 & \text { ng } & \text { vector DNA } \\
50-100 & \text { ng } & \text { insert DNA } \\
1 & \mu l & \text { ligation buffer }(10 \mathrm{x}) \\
1 & \mu \mathrm{l} & \text { T4 DNA ligase }(5 \mathrm{U} / \mu \mathrm{l})
\end{array}
$$

Sticky-end ligations were usually carried out at $16^{\circ} \mathrm{C}$ overnight. 


\subsubsection{Isolation of total RNA from tissues and eucaryotic cell lines}

For isolation of total RNA either Total RNA Isolation Reagent (Biomol) or StrataPrep ${ }^{\mathrm{TM}}$ Total RNA Microprep Kit (Stratagene) were used. Reagents were chosen depending on the sample number and amounts of RNA to be isolated. Purified RNA was stored at $-80^{\circ} \mathrm{C}$ for long or at $-20^{\circ} \mathrm{C}$ for short periods.

\subsection{Isolation with Total RNA Isolation Reagent}

Total RNA Isolation Reagent was used to isolate higher amounts of RNA from small numbers of samples.

10-100 mg of tissues or 5-10 × $10^{6}$ of cultured cells were mixed with $1 \mathrm{ml}$ of RNA reagent and homogenised by using a glass-teflon homogeniser. Following homogenisation, samples were stored for 5 minutes on ice and mixed with $0,2 \mathrm{ml}$ of chloroform. After $15 \mathrm{~min}$ centrifugation $\left(4^{\circ} \mathrm{C}, 12.000 \mathrm{x}\right.$ g, fixed angle) the aqueous phase was collected and RNA was precipitated with an equal volume of isopropanol. After two washes with $70 \%$ ethanol, the RNA pellet was dried and resuspended in 50-100 $\mu$ l of DEPC treated $\mathrm{H}_{2} \mathrm{O}$.

\subsection{Isolation of total RNA with StrataPrep ${ }^{\mathrm{TM}}$ Total RNA Microprep Kit}

StrataPrep ${ }^{\mathrm{TM}}$ Total RNA Microprep Kit was used to isolate small amounts of RNA from high number of eucaryotic cell-culture samples. Less than $5 \times 10^{5}$ cells were scraped, washed with PBS and resuspended in $100 \mu \mathrm{l}$ of Lysis buffer supplemented with $0,7 \mu 1 \beta$-mercaptoethanol. Total RNA was isolated according to the manufacturer's instructions and finally eluted with $30 \mu 1$ of Elution Buffer.

\subsubsection{DNase treatment of total RNA}

First, total RNA (approximately $50 \mu \mathrm{g}$ ) was treated with RNase-free DNase I according to the manufacturer's advice. For this, the following reagents were combined, mixed and incubated at $37^{\circ} \mathrm{C}$ for $30 \mathrm{~min}$ :

$100 \mu 1$ of total RNA $(1 \mathrm{mg} / \mathrm{ml})$

$20 \mu \mathrm{l} \quad 10 \times$ DNase I buffer

$10 \mu \mathrm{l}$ DNase I (1 U/ml)

$70 \mu \mathrm{l}$ deionised water 
DNaseI treated RNA samples were purified by phenol/chloroform extraction and ethanol precipitation (2.2.2.6). The pellet was lyophilised (speed-vac) and dissolved in $50 \mu 1$ RNasefree water. $4 \mu 1$ of the RNA sample was tested on a denaturing agarose gel to verify RNA yield and purity.

\subsubsection{Phenol-chloroform extraction and ethanol precipitation}

Contaminating proteins were removed from the nucleic acid samples by addition: firstly of an equal volume of phenol-chloroform and secondly chloroform to the nucleic acids solution, each followed by centrifugation (10 $\mathrm{min}, 10.000 \mathrm{xg})$ to remove organic phases. Nucleic acids were precipitated with 2,6 volume of absolute ethanol and $\mathrm{NaAc}$ (final conc. $0.3 \mathrm{M}$ ), and washed with $70 \%$ ethanol. The dried pellet was re-dissolved in sterile or RNase free water.

\subsubsection{7 cRNA synthesis}

cRNA was synthesised for electrophysiological measurements, which required microinjection of the RNA into Xenopus oocytes for in vivo translation and protein expression. Template DNA used for transcription was subcloned into pSGEM vector (M. Hollman, see 2.1.7). Reaction was performed with the use of T7 RNA polymerase, which enables in vitro synthesis of large amounts of cRNA from linear cDNA templates. T7 RNA polymerase incorporates 5'7MeGpppG (5' cap analogue) during polymerisation, which strongly increases RNA stability. All reaction steps were performed in RNAse-free environment.

Template cDNA $(5 \mu \mathrm{g})$ was linearised using SfiI enzyme (1-2 U/ $\mu \mathrm{g}$ DNA) in a total volume of $50 \mu 1$ for at least 2 hours at $50^{\circ} \mathrm{C}$ and linearisation efficiency was tested on agarose gels. Afterwards, the reaction volume was increased to $200 \mu \mathrm{l}$ and treated with one volume of phenol/chloroform, vortexed and spinned at $14.000 \mathrm{x}$ for $10 \mathrm{~min}$ at $4^{\circ} \mathrm{C}$. DNA was precipitated for $10 \mathrm{~min}$ at $-70^{\circ} \mathrm{C}$ with $1 / 10 \mathrm{v} / \mathrm{v}$ of $4 \mathrm{M} \mathrm{LiAc}$ and $2,5 \mathrm{v} / \mathrm{v}$ of EtOH and centrifuged (30 min, $14.000 \mathrm{xg}$, at $4^{\circ} \mathrm{C}$ ). Pellet was washed with ice cold $70 \% \mathrm{EtOH}$, dried in a speedvac and resuspended in $60 \mu 1$ of DEPC treated water. 
For cRNA synthesis, the reaction mixture consisted of:

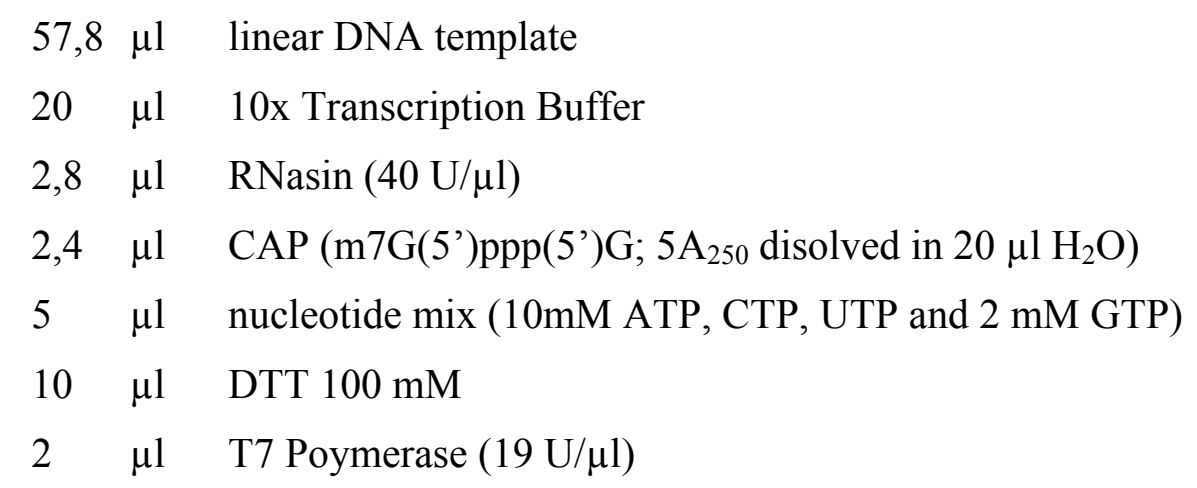

It was incubated at $37^{\circ} \mathrm{C}$ for at least $1,5 \mathrm{~h}$.

The template DNA was removed by digestion with $6 \mu 1$ of DNaseI (RQ1 DNase I, 1U/ $\mu 1)$ at $37^{\circ} \mathrm{C}$ for $15 \mathrm{~min}$. The reaction was stopped by adding $70 \mu \mathrm{l}$ nuclease free water and $30 \mu \mathrm{l}$ of $2 \mathrm{M} \mathrm{NaAc} \mathrm{pH}$ 6,0 followed by phenol/chloroform extraction and ethanol precipitation (overnight in $4^{\circ} \mathrm{C}$ ). Pellet was resuspended in $200 \mu \mathrm{l}$ of $0,3 \mathrm{M} \mathrm{NaAc}$ and ethanol precipitation was repeated $\left(10 \mathrm{~min}\right.$ at $\left.-70^{\circ} \mathrm{C}\right)$. Finally RNA was collected by centrifugation at $14.000 \mathrm{x} \mathrm{g}$ for $30 \mathrm{~min}$ at $4{ }^{\circ} \mathrm{C}$, the cRNA was resuspended in $20-50 \mu 1$ nuclease free water and the concentration was determined. The samples were aliquoted and stored at $-80^{\circ} \mathrm{C}$.

\subsubsection{Spectrophotometric quantitation and estimation of nucleic acid purity}

The concentration of nucleic acids was determined photometrically by measuring absorption of the samples at $260 \mathrm{~nm}$. The ratio A260/A280 was used to estimate DNA purity. For pure DNA is the A260/A280 ratio about 1.8.

\subsubsection{RiboGreen ${ }^{\mathrm{TM}}$ assay for estimation of cRNA concentration}

The absorbance-based method for measuring RNA concentration has some disadvantages like poor sensitivity ( $4 \mu \mathrm{g} / \mathrm{ml} \mathrm{RNA}$ in solution) or interference of the signal with contaminating components such as nucleotides, proteins and salts in the RNA solution. For the injection of Xenopus laevis oocytes exact quantification is needed, since differences in RNA level can have strong effects on the level of synthesised proteins. Thus, the RNA concentration was estimated using RiboGreen ${ }^{\mathrm{TM}}$. The fluorescence of the RiboGreen ${ }^{\mathrm{TM}}$ reagent increases more 
than 1000-fold upon RNA binding and has an emission maximum at $\sim 525 \mathrm{~nm}$. The RiboGreen ${ }^{\mathrm{TM}}$ assay is $\sim 1000$-fold more sensitive than absorbency measurements at $260 \mathrm{~nm}$.

To obtain a standard curve, dilution series of the ribosomal RNA in 1 x TE buffer were prepared. To obtain reliable results the experimental RNA was prepared in six different dilutions. Samples were mixed with 200-times diluted RiboGreen ${ }^{\mathrm{TM}}$ reagent and incubated 5 min at room temperature. Fluorescence was measured in a 96-well plate reader (Wallac Victor; $1 \mathrm{sec}$; Fluorescein filter: excitation $\approx 480 \mathrm{~nm}$, emission $\approx 520 \mathrm{~nm}$ ).

\subsubsection{Polymerase chain reaction (PCR)}

The polymerase chain reaction (PCR) is a very useful technique that allows production of high yields of specific DNA target sequences (Saiki et al., 1988).

PCR was used in this work:

- to amplify specific DNA fragments

- to create appropriate restriction sites at the termini of DNA fragments for cloning into different vectors

- to monitor RNA expression (RT-PCR)

- for in vitro mutagenesis

\subsection{PCR amplification of DNA}

PCR protocols were performed in $50 \mu 1$. A typical $50 \mu 1$ reaction mixture consisted of:

$\begin{array}{lll}10-100 & \text { ng } & \text { plasmid DNA } \\ 10 & \text { pmol } & \text { forward primer } \\ 10 & \text { pmol } & \text { reverse primer } \\ 1 & \mu l & \text { nucleotide mix }(200 \mu \mathrm{M} \text { of each dNTP }) \\ 1 & \mu l & \text { PCR buffer with } \mathrm{MgCl}_{2} \\ 1 & \mathrm{U} & \text { Taq DNA polymerase } \\ \mathrm{x} & \mu \mathrm{l} & \mathrm{ddH}_{2} \mathrm{O}\end{array}$

A 5 min incubation step at $95^{\circ} \mathrm{C}$, during which the template DNA is denatured was followed by $30-40$ cycles of:

$\begin{array}{llll}30-60 & \text { sec } & 95^{\circ} \mathrm{C} & \text { (denaturing) } \\ 30-60 & \text { sec } & 48-63^{\circ} \mathrm{C} & \text { (annealing) } \\ 30-120 & \text { sec } & 68-72^{\circ} \mathrm{C} & \text { (elongation) }\end{array}$


The last cycle was followed by an extra elongation step of 5-10 min at $68-72^{\circ} \mathrm{C}$, depending on the polymerase.

The annealing temperature is dependent on primer composition, on their Tm (melting temperature) and on their homology with the template.

\subsection{One step RT-PCR}

To obtain specific RT-PCR products the Stratagene ProSTAR ${ }^{\text {TM }}$ HF Single-Tube RT-PCR kit was employed. The kit contains optimised components that allow both reverse transcription and PCR amplification to take place in what is commonly referred to as a "one-step" reaction.

The following components were combined for $50 \mu 1$ experimental reaction:

$\begin{array}{lll}\mathrm{x} & \mu \mathrm{l} & \text { RNase-free water } \\ 5 & \mu \mathrm{l} & 10 \mathrm{x} \text { HF RT-PCRbuffer } \\ 10 & \text { pmol } & \text { upstream primer } \\ 10 & \text { pmol } & \text { downstream primer } \\ 1 & \mu \mathrm{l} & \text { dNTP mix }(40 \mathrm{mM}) \\ 0,1-1 & \mu \mathrm{g} & \text { RNA } \\ 1,25 & \mathrm{U} & \text { MMLV-RT } \\ 0,5 & \mu \mathrm{l} & \text { TaqPlus Precision DNA polymerase }\end{array}$

Samples were mixed, placed in the thermal cycler and cycled using the following RT-PCR program:

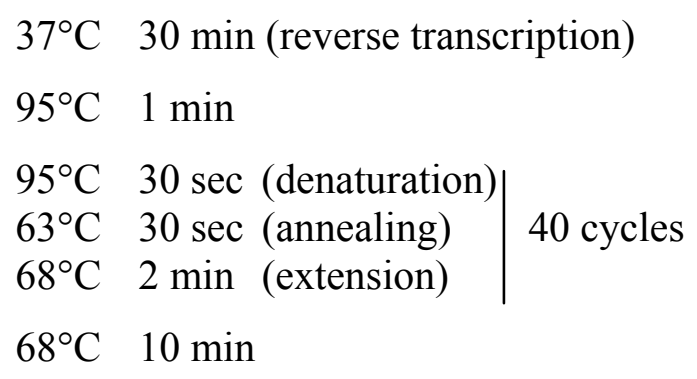

\subsection{Site directed in vitro mutagenesis}

In vitro mutation of targeted nucleotides allows amino acid exchanges, generation or disruption of restriction enzyme recognition sites or correction of errors introduced by PCR amplification. The PCR based, Quick-Change ${ }^{\mathrm{TM}}$ Site-Directed Mutagenesis Kit (Stratagene) was used to create point mutations in the hEag1 cDNA. Pfu polymerase copies the entire 
plasmid from two complementary primers, both of which contain the desired mutation. The mutation was positioned as close to the middle of the primer as possible, with at least 4 bases of perfectly annealing sequence on both sides. The oligonucleotide primers, each complementary to opposite strands of the vector, are extended during temperature cycling by the DNA polymerase. Their incorporation in the synthesised DNA generates a mutated plasmid. After temperature cycling, the PCR product is treated with DpnI, to digest the methylated non-mutated parental DNA template. The nicked vector DNA carrying the desired mutations was then transformed into E. coli.

\begin{tabular}{lll}
\hline Mutation & Forward primer & Reverse primer \\
\hline N26Q & GTTCGGCGGTCCCAGGATACTAATTTTG & CAAAATTAGTATCCTGGGACCGCCGAAC \\
N238Q & CAGCCATCTTGGTCCCTTATCAGGTCTCC & GGAGACCTGATAAGGGACCAAGATGGCTG \\
N388Q & GACACCAAGACAATCCGCCAGAACAGC & GCTGTTCTGGCGGATTGTCTTGGTGTC \\
N406Q & CAGTTTCAGGGGTCTGGCTCAGGGAAG & CTTCCCTGAGCCAGACCCCTGAAACTG \\
N470Q & CATCTTCGGGCAGGTGACGACTATTTTCC & GGAAAATAGTCGTCACCTGCCCGAAGATG \\
N752Q & GCATGCCTCCGCCCAGCACAGCCTCG & CGAGGCTGTGCTGGGCGGAGGCATGC \\
\hline
\end{tabular}

Table 1 Primers used to generate hEag1 mutants

$\underline{\text { PCR sample reaction mix: }}$

$5 \mu \mathrm{l} \quad$ PCR Buffer 10x

$1 \quad \mu \mathrm{l} \quad$ dsDNA template (hEag1 in pTracer, $50 \mathrm{ng} / \mu \mathrm{l}$ )

$1 \quad \mu l \quad$ Primer mix (10 pmol/ $\mu 1$ each $)$

$1 \mu \mathrm{d} \quad \mathrm{dNTP}$ mix

$41 \mu \mathrm{l} \quad \mathrm{ddH}_{2} \mathrm{O}$

$1 \mu 1 \quad$ Pfu Turbo DNA polymerase $(2,5 \mathrm{U} / \mu \mathrm{l})$

Each sample was cycled using the following parameters:

\begin{tabular}{ll|l}
$95^{\circ} \mathrm{C}$ & $30 \mathrm{sec}$ & \\
$95^{\circ} \mathrm{C}$ & $30 \mathrm{sec}$ & \\
$55^{\circ} \mathrm{C}$ & $60 \mathrm{sec}$ & 16 cycles \\
$68^{\circ} \mathrm{C}$ & $18 \mathrm{sec}$ &
\end{tabular}

and chilled to $4^{\circ} \mathrm{C}$ 
Methylated, nonmutated parental dsDNA was removed in each amplification reaction mix by $1 \mathrm{~h}$ incubation $\left(37^{\circ} \mathrm{C}\right)$ with $1 \mu \mathrm{l}$ of DpnI $(10 \mathrm{U} / \mu \mathrm{l})$. Afterwards, amplification was tested by electrophoresis of $5 \mu \mathrm{l}$ of the digested PCR reactions on the $0,8 \%-1 \%$ agarose gels.

For the amplification of the mutated DNA, 2-5 $\mu$ l of each digested PCR products were transformed into XL1-Blue E.Coli.

\subsubsection{Agarose gel electrophoresis}

\subsection{Agarose gel electrophoresis of DNA}

0.5 to $2 \%(\mathrm{w} / \mathrm{v})$ agarose in $100 \mathrm{ml}$ of TBE buffer was boiled in the microwave and poured into a horizontal gel chamber. Gels were then placed into an electrophoresis chamber filled with TBE buffer. 10-20 $\mu 1$ of DNA samples were mixed with $5 \mu 1$ of stop mix applied on gel and run at 50-100 $\mathrm{V}$.

\section{$\underline{\text { TBE buffer }(5 x)}$}

$\begin{array}{lll}225 & \mathrm{mM} & \text { Tris } \mathrm{pH} 8.3 \\ 225 & \mathrm{mM} & \text { Boric acid } \\ 10 & \mathrm{mM} & \text { EDTA }\end{array}$

\subsection{Agarose gel electrophoresis of RNA}

(Maniatis and Efstratiadis, 1980)

Gel electrophoresis of RNA was performed on denaturing agarose gels, in the presence of $1,9 \%$ formaldehyde. $0,5 \mathrm{~g}$ of agarose was dissolved in $5 \mathrm{ml}$ of $10 \mathrm{x}$ MOPS/EDTA buffer and $42,4 \mathrm{ml}$ of DEPC water and boiled in the microwave. After cooling to about $50^{\circ} \mathrm{C}, 2,6 \mathrm{ml}$ of formaldehyde (37\%) was added, stirred and poured into a gel chamber. $5 \mu 1$ of each RNA samples $(1 \mu \mathrm{g} / \mu \mathrm{l})$ as well as molecular weight markers were mixed with $25 \mu \mathrm{l}$ of RNAloading buffer and denatured for $15 \mathrm{~min}$ at $65^{\circ} \mathrm{C}$. Samples were chilled on ice and mixed with $1 \mu \mathrm{l}$ of ethidium bromide $(1 \mathrm{mg} / \mathrm{ml})$. The gel was run at $60 \mathrm{~V}$ for $6-8$ hours. 
$\underline{\text { RNA-loading buffer }}$

$750 \mu 1 \quad$ Formamide

$150 \mu 1 \quad$ MOPS 10x

$240 \quad \mu 1 \quad$ Formaldehyde $37 \%$

$100 \mu 1 \quad$ Glycerol

$3 \mu \mathrm{l} \quad \mathrm{H}_{2} \mathrm{O}$

$20 \mu \mathrm{l} \quad$ Bromphenolblue

$20 \mu 1 \quad$ Xylencyanol

\section{$\underline{\text { MOPS/EDTA }}$}

$\begin{array}{lll}200 & \mathrm{mM} & \text { MOPS } \\ 50 & \mathrm{mM} & \mathrm{NaAc} \\ 10 & \mathrm{mM} & \text { EDTA } \\ \mathrm{pH}=7,0 & \end{array}$

\subsubsection{Biochemical methods}

\subsubsection{Isolation of total proteins from mammalian cells}

For preparation of total proteins cells were cultured in 1-5 Petri-dishes $(\varnothing=17 \mathrm{~cm})$ until 80 $90 \%$ confluency. Cells were washed with PBS, collected with a cell scraper and transferred to separate $50 \mathrm{ml}$ Falcon tubes. After 5 min centrifugation at $1.000 \mathrm{xg}$, cell pellets were washed with $1 \mathrm{ml}$ of PBS, transferred to Eppendorf cups and centrifuged again. Finally cells were lysed with $0,5-1 \mathrm{ml}$ of Lysis buffer for $20 \mathrm{~min}$ in RT and cell debris was removed by $5 \mathrm{~min}$ centrifugation at $10.000 \mathrm{x}$ g. Protein content was measured and the supernatant was used as whole cell lysate for western blotting or stored at $-20^{\circ} \mathrm{C}$.

$\underline{\text { Lysis buffer }}$
$20 \mathrm{mM}$ Tris $\mathrm{pH} 7.5$
1 mM EDTA
$1 \% \quad$ SDS 


\subsubsection{Determination of protein concentration}

Protein concentrations were estimated according to the Bicinchoninic Acid method (BCA; Pierce). 2-5 $\mu \mathrm{l}$ of a protein solution of unknown concentration in $100 \mu \mathrm{l}$ of $\mathrm{H}_{2} \mathrm{O}$ was mixed with $400 \mu \mathrm{l}$ of BCA reagent and incubated for $15-20 \mathrm{~min}$ at $60^{\circ} \mathrm{C}$ under vigorous shaking. Absorbance was measured at $595 \mathrm{~nm}$ and plotted against a reference curve obtained with known concentrations of BSA.

\subsubsection{TCA precipitation}

The BCA test is compatible with many detergents in concentrations up to $1 \%$, however there are substances including reducing agents that interfere with the BCA assay. To eliminate the interfering soluble substances, a TCA precipitation step was performed prior to the BCA test. For this 1-5 $\mu \mathrm{l}$ of the protein solution, as well as standard and blank were mixed with $\mathrm{H}_{2} \mathrm{O}$ to a total volume of $500 \mu 1$. After addition of $100 \mu 1$ of the sodium deoxycholate reagent and 100 $\mu 1$ of the TCA reagent samples were incubated for $10 \mathrm{~min}$ at room temperature and centrifuged for $10 \mathrm{~min}$ at $14.000 \mathrm{x}$ g. Following aspiration of the supernatant the pellet was resuspended in $100 \mu \mathrm{l}$ of $\mathrm{H}_{2} \mathrm{O}$, mixed with $100 \mu \mathrm{l}$ of sodium deoxycholate reagent and $100 \mu \mathrm{l}$ of the TCA reagent. The precipitation step was repeated. Finally, the protein-containing pellet was dissolved in $50 \mu \mathrm{l}$ of SDS reagent, and protein concentration was measured with standard BCA method as described (2.2.3.2).

$\underline{\text { sodium deoxycholate reagent }}$

$0,15 \% \quad$ sodium deoxycholate

\section{$\underline{\text { TCA reagent }}$}

$72 \%(\mathrm{w} / \mathrm{v})$ Trichloroacetic acid

\section{$\underline{\text { SDS reagent }}$}

$$
\begin{array}{lll}
5 & \%(w / v) & \text { SDS } \\
0.1 & \mathrm{M} & \mathrm{NaOH}
\end{array}
$$




\subsubsection{Immunoprecipitation}

Immunoprecipitation is a procedure by which peptides or proteins that react specifically with an antibody are separated from solution and can be used for further studies. Immunoprecipitation is based on the binding of immunoglobulins to bacterial protein $\mathrm{A}$ or protein G. The immobilisation of Protein A/ Protein G on agarose beads allows pulling down of the bound antibody. When incubated with cell lysate, immobilised antibody forms complexes with an antigen that can be easily isolated by a simple centrifugation step.

For coupling of the antibody to form antibody-conjugated beads 5-10 $\mu \mathrm{g}$ of antibody was incubated overnight with

$$
\begin{array}{lll}
25-45 & \mu 1 & \text { Protein G/A- or Protein A-agarose } \\
10 & \mu 1 & 0,1 \% \text { BSA } \\
0,5 & \text { ml } & \text { PBS }
\end{array}
$$

Subsequently beads were washed $3 \mathrm{x}$ with $1 \mathrm{ml}$ wash buffer (10 min each) and finally pelleted (in a benchtop centrifuge for $1 \mathrm{~min}$ at top speed).

Cell lysates were prepared from 1-5 (depending on the purpose) of $\varnothing 16 \mathrm{~cm}$ culture-flasks containing confluent cells. Cells were scraped, washed $3 \mathrm{x}$ with PBS and lysed with $0,5 \mathrm{ml}$ Nondenaturating lysis buffer for 20-30 $\mathrm{min}$ at room temperature. Non-lysed cells were removed by centrifugation $(10 \mathrm{~min}, 14.000 \mathrm{x} \mathrm{g})$ and the final cell lysates were mixed with the prepared antibody conjugated beads and incubated overnight. Afterwards, the proteinantibody-bead complexes were washed 3x with IP-wash buffer. For western blot analysis proteins were removed from beads by addition of $10 \mu 1$ Laemmli buffer and 10 min incubation at $99^{\circ} \mathrm{C}$. For glycosidase treatment, proteins were released from beads by addition of $10 \mu \mathrm{l}$ of $0,1 \% \mathrm{SDS} / 0,1 \mathrm{x}$ Methanol and $10 \mathrm{~min}$ incubation in $99^{\circ} \mathrm{C}$.

\section{$\underline{\text { Nondenaturating lysis buffer }}$}

$\begin{array}{lll}1 & \% & \text { Triton } \mathrm{X}-100 \\ 50 & \mathrm{mM} & \text { Tris } \mathrm{HCl} \mathrm{pH} \mathrm{7,4} \\ 300 \mathrm{mM} & \mathrm{NaCl} \\ 5 & \mathrm{mM} & \text { EDTA }\end{array}$


$\underline{\text { IP-wash buffer }}$

$\begin{array}{lll}0,1 & \% & \text { Triton } \mathrm{X}-100 \\ 50 & \mathrm{mM} & \text { Tris } \mathrm{HCl} \mathrm{pH} \mathrm{7,4} \\ 300 \mathrm{mM} & \mathrm{NaCl} \\ 5 & \mathrm{mM} & \text { EDTA }\end{array}$

\subsubsection{Endoglycosidase treatment}

To monitor carbohydrate chain presence and modifications, hEag1 and mutant proteins were treated with different endoglycosidases. For this, cells were grown in three $75 \mathrm{~cm}^{2}$ culture flasks until they reached confluency. Cells were harvested, lysed in Nondenaturating lysis buffer and protein concentration was determined. 9,5 $\mathrm{mg}$ of cellular proteins were subjected to a standard immunoprecipitation protocol using $20 \mu \mathrm{g}$ of $\mathrm{mAb}-62$ antibody. Immunoprecipitated protein-beads were washed and proteins were released from the beads by incubation with $20 \mu \mathrm{l}$ of $0,1 \mathrm{M} 2$-Mercaptoethanol/0,1 \% SDS (10 min at $\left.99^{\circ} \mathrm{C}\right)$. Beads were removed by a brief spinning and protein-containing supernatant used either for Endoglycosidase H (Endo H) or Peptide N Glycosidase F (PNGaseF) treatment.

\begin{tabular}{lll}
\hline Enzyme & oligosaccharide selectivity & Monitors \\
\hline EndoH & recognises the core region of oligosaccharide chains & ER to Cis-Golgi \\
& on high mannose and hybrid, but no complex & \\
& oligosaccharides & \\
PNGase F & liberates nearly all known N-linked oligosaccharides & all ER and Golgi \\
& from glycoproteins & \\
\hline
\end{tabular}

Table 2 Sensitivity of oligosaccharides to different endoglycosidases Modified, from Coligan et al., 1995 


\subsection{PNGaseF treatment}

To digest glycoproteins with PNGase F $10 \mu \mathrm{l}$ of immunoprecipitated denatured protein in 0,1 M 2-Mercaptoethanol/0,1 \% SDS were incubated overnight in $37^{\circ} \mathrm{C}$ with:

$3 \mu \mathrm{l} \quad 0,5 \mathrm{M}$ Tris- $\mathrm{HCl} \mathrm{pH} 8,5$ (final concentration $60 \mathrm{mM}$ )

$2 \mu 1 \quad 10 \%$ Triton X-100 (final concentration $0,8 \%$ )

$5 \mu \mathrm{l} \quad \mathrm{H}_{2} \mathrm{O}$

$5 \mu \mathrm{l}$ Peptide-N-Glycosidase F (substituted with 0,5 M Tris-HCl pH 8,5 in the control reaction)

\subsection{Endoglycosidase $\mathrm{H}$ treatment}

To digest glycoproteins with PNGase F $10 \mu \mathrm{l}$ of immunoprecipitated denatured protein in 0,1M 2-Mercaptoethanol/0,1\% SDS were incubated overnight at $37^{\circ} \mathrm{C}$ with:

$3 \mu \mathrm{l} \quad 0,5 \mathrm{M}$ sodium citrate, $\mathrm{pH} 5,5$ (final concentration $75 \mathrm{mM}$ )

$20 \mu \mathrm{l} \quad \mathrm{H}_{2} \mathrm{O}$

$2 \mu 1 \quad 1 \%$ PMSF (final concentration $0,05 \%$ )

$1 \mu 1 \quad$ EndoH (substituted with water in the control reaction)

Both reactions were stopped by addition of $6 \times$ Laemmli buffer and products were separated on $7 \%$ SDS-PAGE gels and analysed by immunoblotting.

\subsubsection{Polyacrylamide gel electrophoresis (SDS-PAGE)}

To separate a large range of proteins of varying molecular masses, one dimensional polyacrylamide gel electrophoresis under denaturing conditions (in presence of $0.1 \%$ SDS) was performed.

Two gels: a 'stacking gel' with a low level of cross-linkage and low $\mathrm{pH}$, allowing proteins to enter the gel and collect without smearing, and a 'resolving gel' with a higher $\mathrm{pH}$, in which the proteins are separated according to molecular weights, were poured between two glass plates. For an $8 \times 10$ x $0.1 \mathrm{~cm}$ gel the following volumes were used: 
$10 \mathrm{ml}$ of resolving gel

$\begin{array}{lll}\mathrm{x} & \% & \text { Acrylamide solution }(7-12 \%) \\ 1 & \mathrm{x} & \text { Lower Tris } \\ 0,1 & \% & \text { APS } \\ 0,1 & \% & \text { TEMED }\end{array}$

\section{$5 \mathrm{ml}$ of stacking gel}

$4 \%$ Acrylamide solution

$1 \mathrm{x}$ Upper Tris

$0,1 \%$ APS

$0,1 \%$ TEMED

The resolving gel solution was carefully poured between glass plates, overlayed with isobutanol and allowed to polymerise at room temperature for $30 \mathrm{~min}$. After pouring off the overlaying isobutanol and washing with $\mathrm{ddH}_{2} \mathrm{O}$, the stacking gel solution was poured and a comb was inserted. The gel was allowed to polymerise for further $60 \mathrm{~min}$.

Probes were supplemented with Laemmli sample buffer ( $5 \mathrm{x}$ concentrated), boiled at $95^{\circ} \mathrm{C}$ for $10 \mathrm{~min}$ and loaded on the gel. Prestained molecular weight markers were used as standards. Electrophoresis was run at $50-80 \mathrm{~mA} / 40 \mathrm{~cm}^{2}$ gel, typically for 1-3 h.

\section{Lower Tris $(4 \mathrm{x})$}

$\begin{array}{lll}1,5 & \mathrm{M} & \text { Tris-HCl } \mathrm{pH} 8,8 \\ 0,4 & \% & \text { SDS }\end{array}$

Upper Tris $(4 \mathrm{x})$

$\begin{array}{lll}0,5 & \mathrm{M} & \text { Tris-HCl pH 6,8 } \\ 0,4 & \% & \text { SDS }\end{array}$

Laemmli sample buffer

$\begin{array}{lll}625 \mathrm{mM} & \text { Tris-HCl pH 6,8 } \\ 10 \% & \text { SDS } \\ 16,75 \% & \beta \text {-Mercaptoethanol } \\ 52,5 \% & \text { Glycerin } \\ 0,025 \% & \text { Bromphenol blue }\end{array}$

$\underline{\text { Running buffer }}$

$\begin{array}{lll}192 & \mathrm{mM} & \text { glycine } \\ 25 & \mathrm{mM} & \text { Tris } \\ 0,1 & \% & \text { SDS }\end{array}$




\subsubsection{Commasie brilliant blue staining of proteins in polyacrylamide gels}

Gels were stained in Commasie staining solution for $10 \mathrm{~min}$ up to $1 \mathrm{~h}$. The background was subsequently reduced by soaking the gel in $10 \%$ acetic acid solution and $50 \% \mathrm{MeOH}$ up to several hours. Finally, gels were soaked in $2 \%$ Glycerol, placed in a plastic frame between two cellophane sheets and dried overnight at room temperature.

\section{Commasie staining solution}

$0,1 \%$ Commasie Brilliant Blue R

$10 \%$ acetic acid

$50 \%$ Methanol

\subsubsection{Western blotting and immunological detection of proteins on nitrocellulose filters}

\subsection{Transfer}

After separation by SDS-PAGE proteins were electrophoretically transferred onto nitrocellulose membranes as described (Towbin et al. 1979). Transfer was carried out in Transfer buffer. For hEag1 the $\mathrm{pH}$ of Transfer buffer was adjusted to 10,0 (before addition of SDS and methanol), as the calculated isoelectrical point for Eag is close to the $\mathrm{pH}$

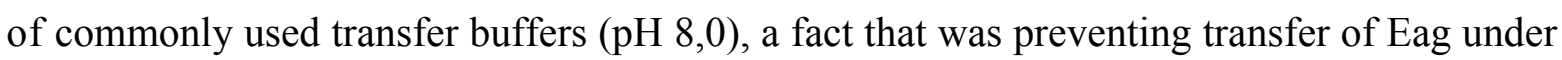
standard conditions.

After electrophoresis of proteins on a polyacrylamide gel, the gel and the nitrocellulose membrane Hybond-ECL (Amersham), which was cut to the size of the gel, were equilibrated in Transfer buffer for 10-30 minutes. Four sheets of Whatman ${ }^{\mathrm{TM}}$ paper were also cut to size and soaked in Transfer buffer. A transfer 'sandwich' (sponge, 2 sheets of paper, running gel, nitrocellulose membrane, 2 sheets of paper, sponge) held together by a plastic cassette, was assembled submerged in transfer buffer to minimise trapping of air bubbles. The cassette was inserted in the buffer tank and transfer was run at $250 \mathrm{~mA}$ constant current for 3-4 hours, or at $100 \mathrm{~mA}$ over night at $4^{\circ} \mathrm{C}$. 
$\underline{\text { Transfer buffer }}$

$\begin{array}{lll}25 & \mathrm{mM} & \text { Tris } \\ 192 & \mathrm{mM} & \text { Glycine } \\ 0,1 & \% & \text { SDS } \\ 10 & \% & \text { Methanol }\end{array}$

\subsection{Ponceau staining}

Transfer of proteins from SDS-polyacrylamide gels was monitored either by Commasie staining of gels after blotting (see 2.2.3.7) or staining of blot-transfer membranes with Ponceau S solution.

Following protein transfer, the nitrocellulose membrane was placed in Ponceau S solution for $5 \mathrm{~min}$ at room temperature. For de-staining, the membrane was placed in $\mathrm{ddH}_{2} \mathrm{O}$ until the background appeared white (2-10 $\mathrm{min})$.

\section{$\underline{\text { Ponceau S solution }}$}

$\begin{array}{lll}2,5 & \mathrm{~g} & \text { Ponceau S } \\ 40 & \% & \text { Methanol } \\ 15 & \% & \text { Acetic acid (glacial) }\end{array}$

\subsection{Antibody detection and enhanced chemiluminescent (ECL) assay for protein visualisation}

Immobilised proteins were probed with specific mouse monoclonal, or rabbit polyclonal antibodies to identify and quantitate antigens. Specific binding was detected by a HRP (Horseradish-peroxidase) conjugated antibody directed against either mouse or rabbit IgG. First, all unspecific binding sites were covered with a non-reactive protein. For this, the membrane was incubated with blocking buffer (5\% non-fat dry milk in PBS) for at least 30 min. Blocking was followed by incubation with an antibody directed against the antigen of interest (primary antibody) diluted in $0,5 \%$ non-fat dry milk/PBS. Incubation was performed for at least 2 hours, or overnight at $4^{\circ} \mathrm{C}$. The membrane was washed three times (10 to 15 min each) with PBST and incubated with the appropriate peroxidase-bound secondary antibody for 1-4 hours at room temperature, or overnight at $4{ }^{\circ} \mathrm{C}$. To remove the non-specific binding of antibodies, several washing steps with PBST, 10-15 min each, were performed. Finally, the proteins were visualised by chemiluminescence. Detection was performed using 
the ECL detection system as recommended by the manufacturer. The exposure of autoradiographic films was performed in a dark room using a film cassette to insure a tight fit between membrane and film. Usually, $1 \mathrm{sec}$ up to $30 \mathrm{~min}$ exposure was performed.

\section{$\underline{\text { PBST }}$}

$$
\begin{aligned}
& 1 \times \text { PBS } \\
& 0,05 \%(v / v) \text { Tween } 20
\end{aligned}
$$

\subsubsection{Subcellular fractionation}

\subsection{Fractionation by differential centrifugation}

To analyse the distribution of a protein in different organelles subcellular fractionation by differential centrifugation in a series of spins of increasing centrifugal force was performed. For this, cells were grown in four Petri-dishes $(\varnothing 17 \mathrm{~cm})$ until they reached confluency, washed with $5 \mathrm{ml}$ of PBS and harvested using a cell-scraper. Cells were transferred to a falcon tube and washed twice with PBS. To disrupt the cells, osmotic shock in $1 \mathrm{ml}$ of Hypotonic Homogenisation Buffer supplemented with protease inhibitors (Roche, 1 tab for $10 \mathrm{ml}$ ) was used. After incubation on ice for $15 \mathrm{~min}$, cells were additionally homogenised by passing 10 times up and down through a $23 \mathrm{G} 1$ needle $(0,6 \times 25 \mathrm{~mm})$ and finally sonicated for $5 \mathrm{~min}$ on ice. Proteins were stabilised by addition of $2 \mathrm{M}$ saccharose. Unbroken cells were removed by $10 \mathrm{~min}$ centrifugation at $100 \mathrm{x}$ g at $4^{\circ} \mathrm{C}$ and the supernatant was collected. Samples were then subjected to further centrifugation steps performed all at $4{ }^{\circ} \mathrm{C}$ :

$$
\begin{array}{ll}
10 \mathrm{~min} \text { at } 1.000 \mathrm{xg} & \text { (P-1 pellet) } \\
10 \mathrm{~min} \text { at } 10.000 \mathrm{xg} & \text { (P-10 pellet) } \\
10 \mathrm{~min} \text { at } 100.000 \mathrm{xg} & \text { (P-100 pellet) }
\end{array}
$$

Pellets were resuspended in $40 \mu \mathrm{l}$ of PBS buffer and protein content was determined. Finally, identical amounts of protein samples were subjected to SDS-PAGE, followed by immunoblotting. 


\begin{tabular}{ll}
\hline Pellet & Content \\
\hline P-1 & nuclei, heavy mitochondria, plasma membrane sheets \\
P-10 & small mitochondria, lysozymes, peroxisomes, intact Golgi and Golgi membranes \\
P-100 & all vesicles from ER, plasma membrane, Golgi, endosomes \\
\hline
\end{tabular}

Table 3 Distribution of cellular organelles in different centrifugation steps. Modified, from Spector et al., 1998

Hypotonic Homogenisation Buffer:

$\begin{array}{llll}20 & \mathrm{mM} & \text { Tris/HCl } & \mathrm{pH} \mathrm{7,9} \\ 2 & \mathrm{mM} & \text { DTT } & \\ 10 & \mathrm{mM} & \text { EDTA } & \\ \mathrm{M} & \text { PMSF } & & \end{array}$

\subsection{Subcellular fractionation by velocity sedimentation on sucrose gradient}

\section{A) Preparation of frozen step-gradient tubes:}

Eleven sucrose solutions were prepared containing respectively, 12, 14, 16, 18, 20, 22, 26, $30,34,38,42,46,50,54$ and $60 \%(w / v)$ sucrose. Starting with the $60 \%$ sucrose solution, a $200 \mu \mathrm{l}$ aliquot was loaded onto the bottom of a $4 \mathrm{ml}$ centrifuge tube, the tube was immersed in liquid nitrogen until the solution was frozen, then $200 \mu \mathrm{l}$ of the next solution was added. The freezing procedure was repeated for all layers. Gradients were stored at $-80^{\circ} \mathrm{C}$. Four to five hours prior to use, the gradients were transferred to $4^{\circ} \mathrm{C}$ for slow thawing.

$\underline{\text { Sucrose solution }}$

$\begin{array}{llll}\mathrm{x} & \% & \text { sucrose } & \\ 10 & \mathrm{mM} & \mathrm{HEPES} & \mathrm{pH} \mathrm{7,5} \\ 1 & \mathrm{mM} & \mathrm{MgCl}_{2} & \end{array}$

\section{B) Fractionation on sucrose gradient}

Cells grown in five Petri-dishes (Ø $17 \mathrm{~cm}$ ) were harvested, washed twice with PBS and collected by centrifugation $(5 \mathrm{~min}$ at $2.000 \mathrm{x} \mathrm{g}$ ). The pellet was resuspended in $0,5 \mathrm{ml}$ hypotonic solution containing highly concentrated protease inhibitors (1 tab pro $1 \mathrm{ml}$ ) and incubated for $20 \mathrm{~min}$ on ice. Cell disruption was checked under the microscope and the 
whole lysates were loaded carefully onto sucrose-gradients. Tubes were centrifuged for 3 hours at $140-180 \times \mathrm{g}$ (swinging bucket) at $4^{\circ} \mathrm{C}$ in an ultracentrifuge and 18 fractions of $200 \mu \mathrm{l}$ each were carefully collected. $50 \mu \mathrm{l}$ aliquots were taken from each fraction, supplemented with Laemmli buffer and analysed by SDS-PAGE and by immunoblotting. Golgi proteins are expected in 10-44\%, plasma membrane sheets in 37-60\%, Golgi in $10-44 \%$, intact nuclei in $60 \%$ and nuclear membranes in $10-55 \%$ sucrose fractions (Spector et al. 1998).

\subsubsection{Eucaryotic cell culture methods}

\subsubsection{Cell culture conditions}

CHO cells were grown in F-12 medium supplemented with $10 \%$ fetal calf serum. Lec1 cells were grown in MEM Alpha Medium supplemented with 10\% fetal calf serum. For culturing cells stably transfected with pTracer and pCDNA4 constructs media were supplemented with Zeocin at $100 \mu \mathrm{g} / \mathrm{ml}$ to maintain selection pressure. The cells were cultivated at $37^{\circ} \mathrm{C}$ in a humidified atmosphere under $5 \% \mathrm{CO}_{2}$ and grown up to about $80 \%$ confluency. For cultivation of slowly growing transfected cells or low confluent cell lines a conditioned, sterile-filtered medium was used.

\subsubsection{Harvesting eucaryotic cells}

The following method is a gentle treatment to remove cells from the substratum while maintaining cellular integrity. First, the exhausted culture medium was removed and cells were washed twice with sterile PBS and incubated in $1 \mathrm{ml}$ of accutase at $37^{\circ} \mathrm{C}$ until they had detached from the dish. The process was controlled under an inverted microscope. The enzyme was inhibited by addition of growth medium in which the cells were subsequently resuspended by pipetting up and down several times until a single-cell suspension was obtained. Single cells were monitored under the microscope and, when necessary, counted in improved Neubauer chamber and plated in the desired dilution. Splitting was performed 2-3 times a week. 


\subsubsection{Cryoconservation of eucaryotic cells}

Cells were detached from the substrate with accutase, centrifuged ( $1.200 \mathrm{x}$ g for $2 \mathrm{~min}$ at RT) and resuspended in 0,5-2 $\mathrm{ml}$ of complete growth medium $\left(1-5 \times 10^{6}\right.$ cells $\left./ \mathrm{ml}\right)$. Cell suspension was gently mixed with an equal volume of freezing medium and aliquoted into storage vials. Vials were placed in an insulated box in a $-80{ }^{\circ} \mathrm{C}$ freezer overnight. Finally, the vials were stored in liquid nitrogen.

\section{Freezing medium}

$\begin{array}{lll}25 & \% & \text { FCS } \\ 25 & \% & \text { DMSO }\end{array}$

in appropriate culture medium (Ham's F12 or Alpha Modified Eagle's Medium)

\subsubsection{Thawing and revitalising of cryopreserved cells}

Cryopreserved cells are fragile and require gentle handling. The cells were quickly thawed in a $37^{\circ} \mathrm{C}$ water bath after being removed from liquid nitrogen. Then, cells were plated directly into a flask containing pre-warmed complete culture medium. Cells were allowed to attach for 3 hours in the incubator. Thereafter the DMSO-medium was removed by gentle suction and addition of fresh medium containing appropriate antibiotics.

\subsubsection{Mycoplasma test}

Mycoplasma are frequently found contaminants in cell cultures. Contamination with mycoplasma usually does not result in turbid growth within cultures or macroscopic alteration of the cells. However, mycoplasma contamination may cause adverse effects, such as changes in metabolism, growth rate, viability, DNA, RNA and protein synthesis, morphology etc., which lead to unreliable experimental results. Therefore, cell cultures were regularly tested for the presence of mycoplasma using Mycoplasma Plus ${ }^{\mathrm{TM}}$ PCR Primer Set (Stratagene) according to the manufacturer's instructions. 


\subsubsection{Transfection of mammalian cells}

Transfection of $\mathrm{CHO}$ and Lec1 cells was accomplished using DAC-30 (Eurogentec) according to the supplier's instructions. In the later part of the work, DAC-30 was replaced by Fugene (Roche), since this resulted in higher transfection efficiency and became a common transfection reagent in our lab. Cells $\left(2 \times 10^{5}\right)$ were plated out in appropriate growth medium on $\varnothing 10 \mathrm{~cm}$ Petri dishes for 16-24 hours. On the day of transfection, culture medium was replaced by $2,5 \mathrm{ml}$ of fresh medium and appropriate DNA mix was prepared in the following order:

\section{$\underline{\text { DAC-30 mix }}$}

$10 \mu \mathrm{g}$ plasmid DNA

$25 \mu \mathrm{g}$ DAC-30

$2,5 \mathrm{ml}$ serum-free culture medium

\section{Fugene mix}

$194 \mu 1 \quad$ serum-free culture medium

$6 \quad \mu \mathrm{l}$ Fugene reagent

$4 \quad \mu \mathrm{g}$ plasmid DNA

DNA mix was incubated 15-30 min in room temperature and poured drop-wise onto cells. In case of transient transfection, cells were used for analysis after about 48 hours. For production of stable cell lines, Zeocin was added after 48 hours to the culture medium $(100 \mu \mathrm{g} / \mathrm{ml})$ for selection. This was carried out continuously to avoid phenotype recovery.

\subsubsection{Preparation of polylysine-coated tissue culture surfaces}

To ensure better attachment of $\mathrm{CHO}$ cells to the surface, coverslips used for immunofluorescence microscopy and electrophysiological measurements were covered with poly-L-lysine.

Prior to coating, fat impurities possibly existing on glass coverslips were removed. For this 100-200 coverslips were incubated for at least 1 hour in $96 \%$ EtOH under gentle agitation. 
Coverslips were washed several times with water, autoclaved and finally placed in sterile poly-L-lysine solution (handled in sterile environment). After $1 \mathrm{~h}$ incubation with frequent shaking, coverslips were washed several times with sterile PBS. Coated coverslips were stored at room temperature under sterile conditions for up to several weeks.

\subsubsection{Incubation with Tunicamycin}

To estimate the optimal working concentration of Tunicamycin, $\mathrm{CHO}$ cells were plated on 12-well plates in a density, in which they can reach confluency after $48 \mathrm{~h}$ (50.000 cells per well). $24 \mathrm{~h}$ later the medium was changed for fresh F12 medium containing decreased concentrations of Tunicamycin $(10-0,3 \mu \mathrm{g} / \mathrm{ml})$. As a control, the same cells were incubated with the corresponding amounts of DMSO (solvent). Cells were incubated at $37^{\circ} \mathrm{C}$ for $24 \mathrm{~h}$ and survival in Tunicamycin was observed under the microscope. As a working concentration, the highest concentration of Tunicamycin allowing survival of approximately $80 \%$ of cells was used $(2,5 \mu \mathrm{g} / \mathrm{ml})$.

For the internal inhibition of N-linked glycosylation, $\mathrm{CHO}$ cells stably transfected with hEag1-pTracer were harvested and plated at the desired dilution. For western blot analysis cells were plated on $\varnothing 17 \mathrm{~cm}$ Petri-dishes and incubated for about $24 \mathrm{~h}$ until they reached $80 \%$ confluency. For electrophysiological studies and immunofluorescent localisation cells were plated on coverslips placed in Petri-dishes. Low dilution allowing electrophysiological measurements after more than $48 \mathrm{~h}$ was chosen. Several hours after attachment of cells, the culture medium was replaced by inhibitor containing medium $(2,5 \mu \mathrm{g} / \mathrm{ml}$ Tunicamycin $)$ without antibiotics. In the control incubation, Tunicamycin was substituted by DMSO (vehicle). The inhibitor was allowed to act for $24 \mathrm{~h}$ under normal cell culture conditions. Afterwards, cells were used for further studies. 


\subsubsection{Microscopic analysis}

\subsubsection{Immunofluorescent localisation of proteins}

Cells were plated on round $(\varnothing 1,5 \mathrm{~cm})$, sterilised, polylysine coated coverslips $2-3$ days before staining and grown to about 50\% confluency. After removal of the medium cells were washed three times in PBS, fixed in $4 \%$ paraformaldehyde $\left(20\right.$ min at $\left.4{ }^{\circ} \mathrm{C}\right)$ and washed again three times in PBS. Cells were permeabilised for 10 min in $0.1 \%$ Triton X-100/PBS and washed three times in PBS (10 min each). Alternatively, cells were fixed with methanol $\left(20 \mathrm{~min},-20^{\circ} \mathrm{C}\right)$ directly followed by PBS washes. Non-specific antibody binding was blocked by $10 \mathrm{~min}$ incubation in 10\% BSA/PBS solution. Cells were then incubated with the primary antibody in the appropriate dilution and washed three times for $10 \mathrm{~min}$ in PBST. Finally, the appropriate fluorescent-dye labelled secondary antibody in PBS was added to the cells and incubated for $1 \mathrm{~h}$ in the darkness. After three 10 min washing steps in PBST, nuclei were optionally stained with Hoechst 33342 and coverslips were mounted using the ProLong ${ }^{\mathrm{TM}}$ Antifade kit and left at least over night at $4^{\circ} \mathrm{C}$. Microscopic analysis was performed using inverted fluorescence microscope or confocal laser scanning microscope.

\section{$\underline{\text { PBST }}$}

$$
\begin{array}{lll}
1 & \mathrm{x} & \text { PBS } \\
0,05 & \%(\mathrm{v} / \mathrm{v}) & \text { Tween } 20
\end{array}
$$

\subsubsection{Electrophysiological methods}

All electrophysiological measurements were performed in cooperation with Dr. Francisco Monje (MPI Göttingen). 


\subsubsection{Preparation and injection of Xenopus laevis oocytes}

Xenopus laevis oocytes contain only very few endogenous voltage-activated channels and are relatively large (about $1 \mathrm{~mm}$ in diameter) which makes them a very useful system to study heterologously expressed ion channels.

For oocyte extraction from the frog Xenopus laevis, a small surgical opening was performed under anaesthesia (2.5 g/l of Tricaine). After isolation of oocytes frogs the incision was closed immediately and frogs were returned to water.

The follicular cell layer was removed form oocytes by 2-3 hours incubation in collagenase solution. After inhibition of the enzymatic reaction by thoroughly washing the oocytes with Barth medium, oocytes in stages V-VI were isolated with fine forceps for cRNA injection.

Pipettes used for cRNA injection were pulled using a standard pipette puller. Pipettes with an opening of $\sim 10 \mu \mathrm{m}$ in diameter were polished to obtain a needle-like shape which reduces oocytes damage during injection. Microinjection of cRNA ( $\sim 50 \mathrm{nl}$ per oocyte) was performed using an automatic injector. Injected oocytes were incubated at $18^{\circ} \mathrm{C}$ in Barth medium containing penicillin/streptomycin $(100 \mathrm{U} / \mathrm{ml})$ and Cefuxorixim/Zinacef750 (4 mg/l) for 3-7 days prior to electrophysiological analysis.

Barth medium

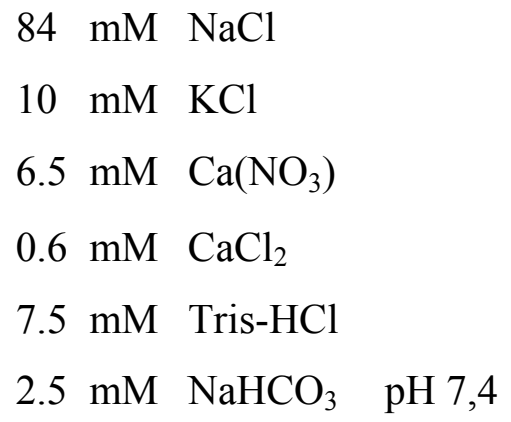

\subsubsection{Two-electrode voltage clamp (TEVC)}

The TEVC is a method used extensively for the study of functional properties of voltagegated ion channels expressed in oocytes (Stühmer, 1992). This method allows an accurate 
measurement of the ionic current through voltage-gated ion channels under adequate control of the membrane potential as well.

When an applied voltage induces opening of channels, the ionic flux tends to alter the membrane potential. Under TEVC control, one intracellular electrode (the voltage electrode) is used for continuous measurement of the actual membrane potential, while the second (the current electrode) is used to pass the current necessary to keep the potential at the desired value (Fig. 4). This is achieved using a feedback circuit, which is the main component of the voltage clamp. The amount of current that passes through the current electrode is the measured parameter.

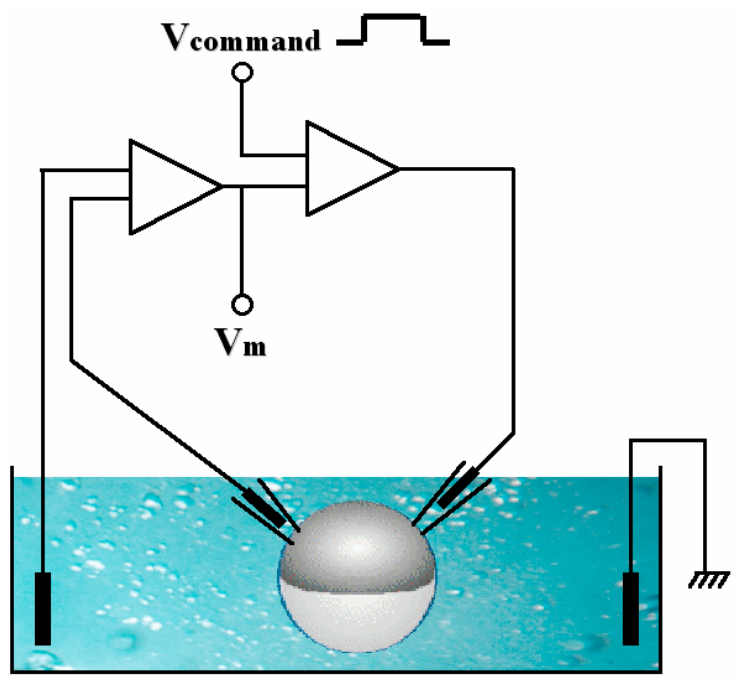

Fig. 4 A schematic diagram of the main components of a two-electrode voltage clamp

The difference in potential between the bath and the potential electrode (left), $\mathrm{V}_{\mathrm{m}}$, is compared to the command potential, $\mathrm{V}_{\text {comm }}$ and determines the amount of current injected into the oocyte through the current electrode (right).

\subsubsection{TEVC stimulation protocols}

The two-electrode voltage clamp pipettes were made from Borosilicate glass capillares, containing a thin internal filament to ensure the complete filling of the electrode, and were filled with $2 \mathrm{M} \mathrm{KCl}$ (internal solution). The tips of the pipettes were broken before use in order to decrease the electrical resistance to the range of 0.4- $0.7 \mathrm{M} \Omega$.

From a holding potential of $-80 \mathrm{mV}$ standard IV protocols, consisting of discrete depolarising pulses of increasing amplitudes, at increments of 10 or $20 \mathrm{mV}$, from the 
holding potential and up to $+80 \mathrm{mV}$ were applied (see Fig. 5). The amplitude of the current elicited during each pulse of voltage (test pulse) depends on the number of channels present in the membrane, their open probability, their conductance and the driving force of the permeating ion species.

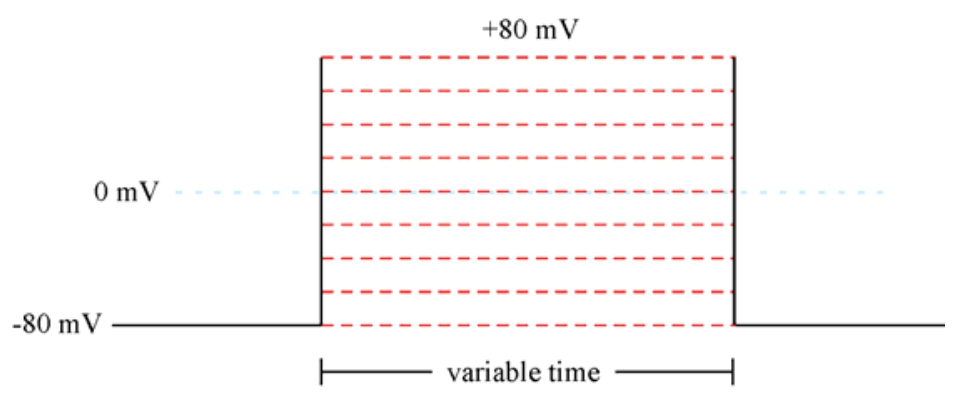

Fig. 5 Standard IV protocol.

Starting from the holding potential from - $80 \mathrm{mV}$, discrete depolarising pulses of variable amplitudes, with increases of 10 or $20 \mathrm{mV}$, were applied during a variable time.

When channels are open under conditions (particularly hyperpolarising voltage and high extracellular potassium concentrations) that cause inward currents, an instantaneous inward tail current is apparent at the end of each depolarising pulse. The peak amplitude of this tail current is proportional to the open probability of the channels at test pulse potential, since the channels start to deactivate only after the change in potential. Since this inward current is always measured at a constant return (or tail) potential, i.e. at a constant driving force, it can be used to determine the voltage dependence of channel opening (activation curve). The activation curve can be characterised by Boltzman functions of the type:

$\mathrm{Po} / \mathrm{Po}_{\max }$ or $\mathrm{I} / \mathrm{I}_{\max }=1-\left(\mathrm{G}_{\max } /\left(1+\exp \left(\mathrm{V}-\mathrm{V}_{1 / 2}\right) / \kappa\right)\right)$

$\mathrm{V}_{1 / 2}$ indicates the potential at which half of the entire population of channels are in the open state. The e-fold slope allows the evaluation of the steepness of the dependence of the open probability on the membrane potential.

Tail currents are also useful to study the kinetics of deactivation. For this, the time curse corresponding to the relaxation of the tail currents is fitted with a single-exponential function using the PulseFit software in order to estimate the time constant of deactivation. 


\section{Normal Frog Ringer (NFR) external solution}

$\begin{array}{lll}85 & \mathrm{mM} & \mathrm{NaCl} \\ 30 & \mathrm{mM} & \mathrm{KCl} \\ 1.8 & \mathrm{mM} & \mathrm{CaCl}_{2} \\ 2 & \mathrm{mM} & \mathrm{MgCl}_{2} \\ 10 & \mathrm{mM} & \mathrm{HEPES}\end{array}$

$\underline{\text { Internal solution }}$

$2 \mathrm{M} \quad \mathrm{KCl}$

\subsubsection{Patch clamp measurements of potassium currents from $\mathrm{CHO}$ cells}

The patch clamp is a voltage clamp in which a determined membrane potential is maintained under a specific area of cell membrane. The flux of transmembrane ionic currents as well as the membrane capacitance can be resolved with great accuracy (Hamill et al. 1981; Marty and Neher 1983).

A patch of membrane is isolated by attaching a clean and smooth tip of a glass pipette on the surface of the cell membrane and obtaining a very tight seal between the pipette and the membrane. The adhesion between the pipette and the membrane can be so good that a tight seal can reach a shunt resistance larger than $10 \mathrm{G} \Omega$.

Application of an additional negative pressure pulse (suction) is used to perforate the membrane located under the pipette leading to the whole-cell configuration (Fig. 6). In the whole-cell mode, electrical and chemical communication between the pipette solution and the intracellular medium is established, which allows an accurate control of the membrane potential and the intracellular chemical composition.

In the experiments described here, the whole-cell configuration of the patch-clamp technique was used, in which channel openings can be induced by varying the membrane potential. In this configuration, the average ionic current flowing through the channels present in the entire cell membrane is recorded. 


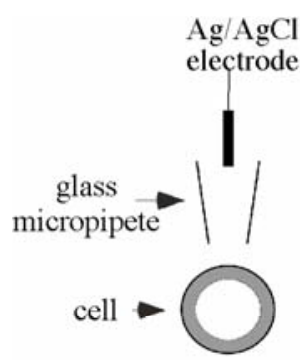

A

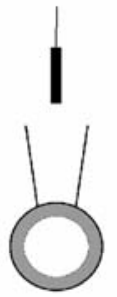

B

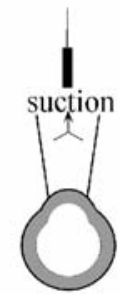

C

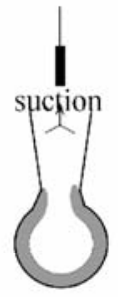

D

$\mathbf{E}$

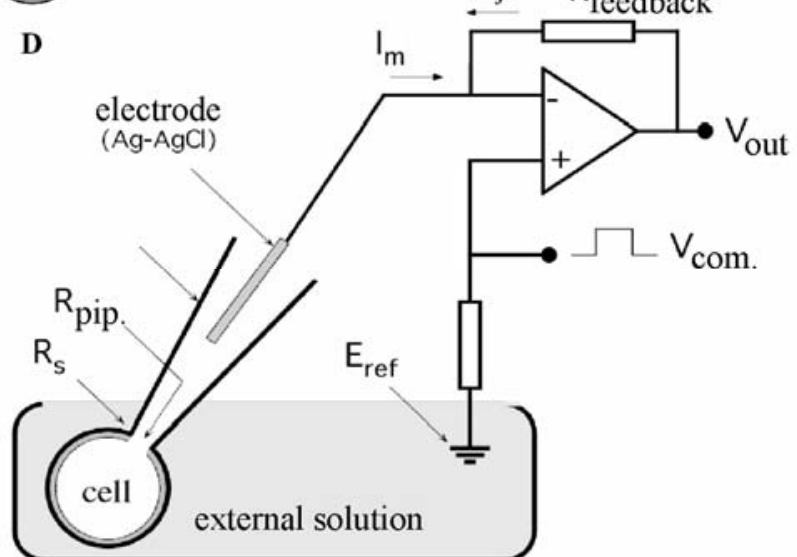

Fig. 6 Simplified diagram of the patch clamp whole-cell configuration

A) A clean and tip-polished glass pipette is approached to the cell

B) The pipette isolates a small area of the cell membrane

C) Gentle suction allows the formation of a very tight seal between the cell membrane and the walls of the pipette, leading to the cell-attached configuration

D) Additional suction breaks the isolated area of membrane leading to the whole cell configuration

E) Schematic diagram of the main components of the patch clamp technique

\subsubsection{Stimulation-acquisition protocols and data analysis}

For patch clamp measurements we used pipettes made of aluminiumsilicate glass capillaries. Pipettes were pulled on a conventional vertical pipette puller and tips were fire-polished under visual control through a light microscope. Resulting pipettes had resistances of 1-3 $\mathrm{M} \Omega$ when filled with the intracellular solution.

In all the experiments, the membrane was kept at a holding potential of $-80 \mathrm{mV}$, where the probability of the hEag1 channels to be in the close state is very high. Standard voltage pulse protocols were applied to elicit and record the ionic currents flowing in response to the activation of the channels. Starting from a holding potential of $-80 \mathrm{mV}$, several depolarising pulses were applied up to $+80 \mathrm{mV}$ in increments of 10 or $20 \mathrm{mV}$ (Fig. 6). The duration of these pulses was between 500 and $2000 \mathrm{~ms}$ (see time scale in corresponding graphs). 
Currents were further filtered at 1-3 kHz, and digital sampling intervals were 100-200 $\mu$ s. Series resistance was systematically compensated up to $60 \%$. Leak and capacitative currents were systematically subtracted on-line using a $\mathrm{P} / \mathrm{n}$ protocol as they essentially depend linearly on the applied potential. Linear leakage currents were estimated at voltage ranges in which the closed-state probability of hEag1 channels was close to 1. Current amplitudes were obtained by averaging the currents recorded from at least 5 different cells, and standard deviation (SD) of the samples used was calculated. In patch clamp experiments, current densities $(\mathrm{pA} / \mathrm{pF})$ for each cell were calculated from the steady-state values of the measured peak-current amplitudes divided by the value of cell membrane capacitance (determined by the automatic $\mathrm{C}_{\text {slow }}$ compensation).

Internal solution
$130 \mathrm{mM} \mathrm{KCl}$
$10 \mathrm{mM}$ HEPES $\mathrm{pH} 7.2$
$10 \mathrm{mM}$ BAPTA

\section{External solution}

$\begin{array}{lll}110 & \mathrm{mM} & \mathrm{NaCl} \\ 30 & \mathrm{mM} & \mathrm{KCl} \\ 2 & \mathrm{mM} & \mathrm{CaCl} 2 \\ 2 & \mathrm{mM} & \mathrm{MgCl} 2 \\ 10 & \mathrm{mM} & \text { D-glucose } \\ 10 & \mathrm{mM} & \text { HEPES }\end{array}$

\subsubsection{Yeast Two-Hybrid System (Y2H)}

(Fields and Song, 1989)

The Yeast Two-Hybrid System allows the detection of protein-protein interactions in vivo based on the fact that many eucaryotic transcription factors exist as two functionally independent domains, which can be separated without loss of function. Thus, the GAL4 DNA-binding domain (BD) and GAL4 activation domain (AD), which are normally part of the same protein, can be expressed separately fused with bait or pray protein. 
Both fusion-proteins are expressed in the same cell in the case of interactions between bait and pray. Thereby both domains (BD and AD) are brought into close proximity and become active, recognising UAS (,upstream activating sequence“) of GAL4-responsive elements and initiating transcription of reporter genes (Fig. 7).

To identify interaction partners of hEag1, MATCHMAKER GAL4 Two-Hybrid System 3 was used. In this system two different S.cerevisiae strains are used:

- AH109, auxotroph for adenine, histidine, leucine and tryptophan and

- Y187, auxotroph for leucine and tryptophan.

Additionally, MEL1 and lacZ reporter genes encode in both species for $\alpha$ - and $\beta$-galactosidase, respectively.

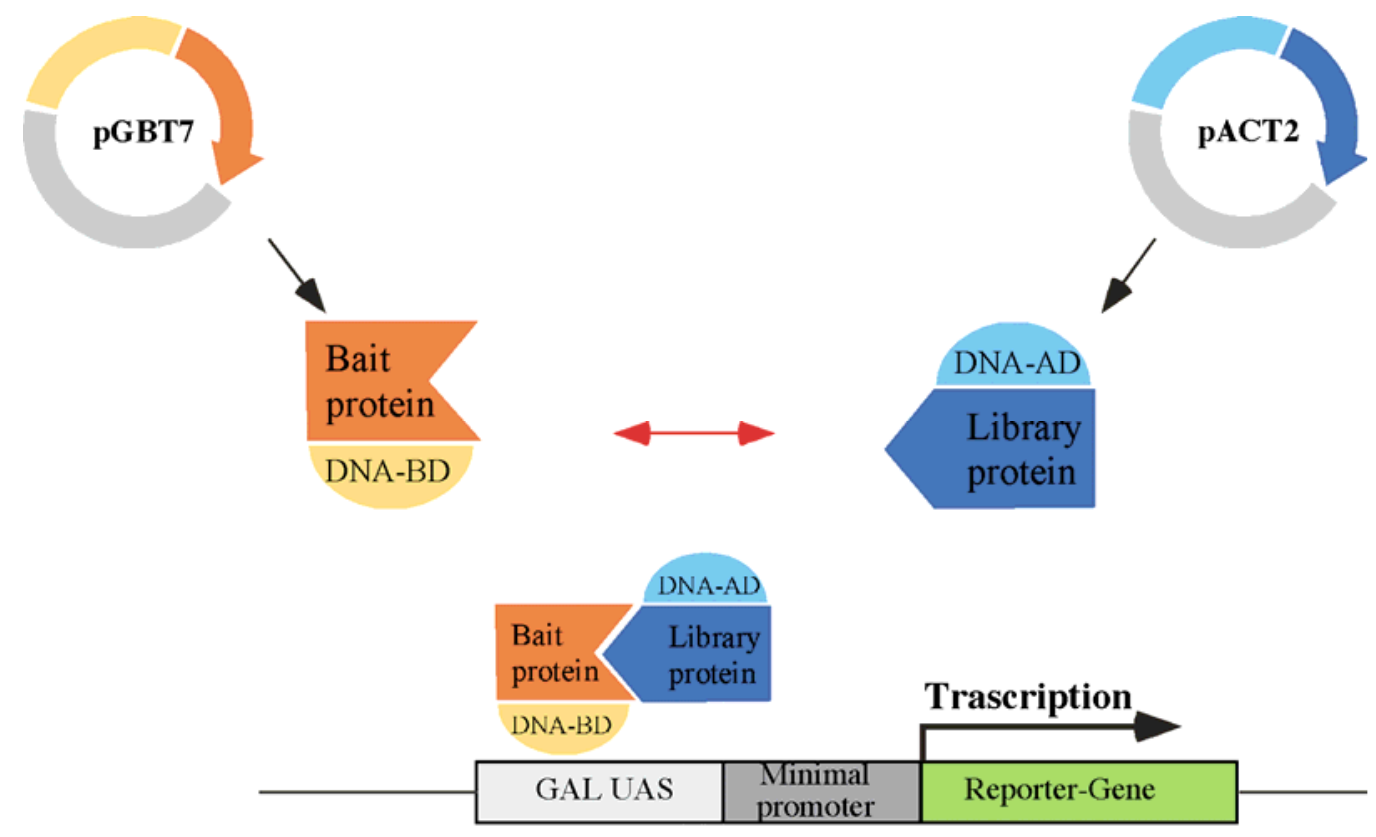

Fig. 7 Yeast Two-Hybrid method

\subsubsection{Transformation of S.cerevisiae AH 109}

S.cerevisiae AH 109 yeast was transformed with pGBKT7 constructs and transformation was carried out using a modified lithium acetate method (Gietz et al., 1995). For 7 transformations, $1 \mathrm{ml}$ of YPDA medium with 3 colonies of AH 109, was vortexed, transferred to $50 \mathrm{ml}$ of YPDA and incubated overnight at $30^{\circ} \mathrm{C}(320 \mathrm{rpm}) .5 \mathrm{ml}$ of this culture were then diluted 1:10 with YPDA medium $\left(\mathrm{OD}_{600} \sim 0.4\right)$ and incubated for $3 \mathrm{~h}$ at $30^{\circ} \mathrm{C}$ 
under vigorous shaking. Cells were collected by centrifugation (500 x g, $5 \mathrm{~min})$, washed with sterile water and centrifuged again. Finally, cell pellet was resuspended in $750 \mu 1$ of freshly prepared TE/LiAc and used as competent cells.

For each transformation the following mix was prepared:

$$
\begin{array}{lll}
0,1 & \mu \mathrm{g} & \text { DNA } \\
100 & \mu \mathrm{g} & \text { DNA carrier (Salmon-sperm DNA) } \\
100 & \mu \mathrm{l} & \text { competent AH } 109 \text { cells } \\
600 & \mu \mathrm{l} & \text { freshly prepared PEG/LiAc }
\end{array}
$$

After $30 \mathrm{~min}$ incubation at $30^{\circ} \mathrm{C}$ with slow agitation $(60 \mathrm{rpm})$ the yeast suspension was substituted with $70 \mu \mathrm{DMSO}$ and gently mixed, followed by 5 min heat-shock at $42^{\circ} \mathrm{C}$ in a water bath. After short incubation on ice, cells were pelleted in a picofuge, resuspended in $0,5 \mathrm{ml}$ of TE buffer and plated on SD -Trp selection plates to isolate positive transformants.

\subsubsection{Yeast mating}

To identify putative hEag1 binding partners the MATCHMAKER ${ }^{\mathrm{TM}}$ pretransformed human cDNA library was used. In this system, the library cDNA is subcloned into the pACT2 vector (pray) and transformed into the Y187-MAT $\alpha$ yeast strain. The bait protein (Cterminus of hEag1) was subcloned into the AH109-MATa, a mating partner for the Y187MAT $\alpha$ strain.

A $50 \mathrm{ml} \mathrm{SD} /$-Trp medium was inoculated with a few colonies of AH 109 hEag1-CT (from SD -Trp plates) and grown overnight in $30^{\circ} \mathrm{C}$ with agitation (OD $\left.\geq 0.8\right)$. Cells were pelleted and resuspended in $50 \mathrm{ml}$ of $2 \mathrm{x}$ YPDA-Kanamycine medium. The pretransformed library was thawn on ice directly before use, added to the suspension and agitated slowly in $30^{\circ} \mathrm{C}$ for 24-28h. Cell culture was washed twice with $50 \mathrm{ml}$ of $2 \mathrm{xY}$ PDA-Kanamycine and cells were resuspended in $10 \mathrm{ml}$ of 0,5 x YPDA-Kanamycine. To select diploids expressing positively interacting proteins, $300 \mu \mathrm{l}$ aliquots were plated on SD -LTHA plates enriched with X- $\alpha-$ Gal and incubated 12 days in $30^{\circ} \mathrm{C}$. Additionally, $100 \mu 1$ aliquot was used for the control of mating efficiency. 


\subsubsection{3 $\quad \beta$-galactosidase assays}

Prior to the $\beta$-galactosidase assays diploids were grown for several days on SD -LT selection plates.

\subsection{Colony-lift filter assay (Breeden and Nasmyth, 1985)}

To duplicate the colonies, a dry Whatman filter was placed over the surface of the plate containing the colonies to be assayed. The filter was then placed for 10 seconds in liquid nitrogen, thawn at room temperature and placed, colony side up, on another Whatman paper pre-soaked in Z-buffer/X-gal solution and both were incubated at $30^{\circ} \mathrm{C}$ for up to 8 hours.

\section{Z buffer}

$$
\begin{array}{lll}
60 & \mathrm{mM} & \mathrm{Na}_{2} \mathrm{HPO}_{4} \\
125 & \mathrm{mM} & \mathrm{NaH}_{2} \mathrm{PO}_{4} \times \mathrm{H}_{2} \mathrm{O} \\
100 & \mathrm{mM} & \mathrm{KCl} \\
10 & \mathrm{mM} & \mathrm{MgSO}_{4} \times 7 \mathrm{H}_{2} 0
\end{array}
$$

\section{$\underline{\text { Z buffer/X-gal solution }}$}

$100 \mathrm{ml} \mathrm{Z}$ buffer

$0.27 \mathrm{ml} \beta$-mercaptoethanol

\section{$\underline{X-g a l ~ s o l u t i o n}$}

$$
20 \mathrm{mg} / \mathrm{ml} \quad \mathrm{X} \text {-gal in DMF }
$$

\subsection{In-plate assay (Duttweiler, 1996)}

Plates containing colonies to be analysed were covered with chloroform and incubated for $5 \mathrm{~min}$ at room temperature. Chloroform was removed and plates were dried for $5 \mathrm{~min}$ at room temperature. $15 \mathrm{ml}$ of the agarose solution was boiled, cooled to about $40^{\circ} \mathrm{C}$ and supplemented with 1,5 $\mathrm{ml}$ of X-gal solution and poured onto the plate. Plates were incubated at $30^{\circ} \mathrm{C}$ up to $24 \mathrm{~h}$, and the appearance of blue colonies was controlled regularly. 
$\underline{\text { Agarose solution }}$

$\begin{array}{lll}200 & \mathrm{mM} & \mathrm{K}_{2} \mathrm{HPO}_{4} \\ 75 & \mathrm{mM} & \mathrm{KH}_{2} \mathrm{PO}_{4} \\ 1 & \% & \text { Agarose }\end{array}$

$\underline{\text { X-gal solution }}$

$20 \mathrm{mg} / \mathrm{ml} \mathrm{X}$-gal in DMF

\subsubsection{Recovery of plasmid DNA from S. cerevisiae}

To recover the library plasmid from diploid yeast, $5 \mathrm{ml}$ of SD/-Leu/-Trp medium was inoculated with a single colony selected to be analysed and incubated 48 hours at $30^{\circ} \mathrm{C}$ with agitation. Cells were pelleted, washed with $1 \mathrm{ml}$ of $1 \mathrm{x}$ TE buffer and resuspended in $200 \mu \mathrm{l}$ of the Yeast lysis buffer-1. Suspension was mixed with $100 \mu \mathrm{l}$ phenol and $100 \mu \mathrm{l}$ of chloroform:isoamyl alcohol (24:1) and $300 \mathrm{mg}$ of glass beads and shaken strongly for 10 $\min$ at RT to break the cells. Afterwards, lysates were centrifuged for $10 \mathrm{~min}$ at $4^{\circ} \mathrm{C}$, $14000 \mathrm{x}$. The upper phase was collected, supplemented with $5 \mu 1$ of 7,5 M ammonium acetate, incubated for $60 \mathrm{~min}$ at $-20^{\circ} \mathrm{C}$ and centrifuged $\left(15 \mathrm{~min}, 4^{\circ} \mathrm{C}, 14000 \mathrm{x} \mathrm{g}\right)$. The supernatant was subjected to EtOH precipitation and DNA pellet was resuspended in $20 \mu 1$ of sterile water.

For amplification, DNA was transformed into competent E.Coli cells. Transformants were selected with medium containing Ampicilin.

$\underline{\text { Yeast lysis buffer-1 }}$

$\begin{array}{lll}2 & \% & \text { Triton } \mathrm{X}-100, \\ 1 & \% & \mathrm{SDS}, \\ 100 & \mathrm{mM} & \mathrm{NaCl}, \\ 10 & \mathrm{mM} & \text { Tris-HCl } \mathrm{pH} 8,0 \\ 1 & \mathrm{mM} & \text { EDTA }\end{array}$




\subsubsection{Isolation of total cellular proteins from S.cerevisiae AH 109}

To isolate total cellular proteins from yeast, $6 \mathrm{ml}$ of medium (YPDA for AH109 and SD-Trp for AH109 GAL4-hEag1CT) were inoculated with a single colony and incubated overnight at $30^{\circ} \mathrm{C}(320 \mathrm{rpm})$. Cells were pelleted ( $\left.5 \mathrm{~min}, 500 \mathrm{x} \mathrm{g}\right)$, washed with sterile water and lysed in $60 \mu \mathrm{l}$ of Yeast lysis buffer-2 for $20 \mathrm{~min}$ at room temperature. Non-lysed cells were removed by centrifugation, and lysates were supplemented with Laemmli loading buffer divided into two equal samples and analysed on 9\% SDS-PAGE.

$\underline{\text { Yeast lysis buffer-2 }}$

$\begin{array}{llll}15 & \mathrm{mM} & \text { TRIS HCl } & \mathrm{pH} \mathrm{7,5} \\ 1 & \% & \text { SDS } & \end{array}$

\subsubsection{Computer analysis}

DNA and protein sequence analysis and alignment in contigs were done with "LASERGENE" (DNASTAR). For multiple sequence alignments " CustalW" was used (available at http://www2.ebi.ac.uk/clustalw/help.html or at http://dot.imgen.bcm.tmc.edu: 9331/multi-align/multi-align.html).

Other sequence analysis tools used are available at the ExPASy site: http://www.expasy.ch/ or at the NPSA (network protein sequence analysis) site: http://pbil.ibcp.fr/. For database sequences search, "WU-Blast2" was used, available at the EBI (European Bioinformatics Institute) site: http://www2.ebi.ac.uk/.

Acquisition and analysis of electrophysilogical data were performed using thePulse+PulseFit (HEKA Elektronik) and IGOR (Wavemetrics) software packages. 
$3 \quad$ RESULTS

\subsection{Biochemical analysis of Eag1 in rat}

\subsubsection{Analysis of the Eag1 protein expression pattern}

The ether á go-go potassium channel was first identified in the neuromuscular junction of Drosophila melanogaster larvae. In mammalian species, Eag1 is mainly expressed in adult brain (human, mouse, rat), but its expression was also demonstrated in some other tissues like human placenta, human testis (Pardo, unpublished), bovine retina or in myoblasts at the onset of fusion. Furthermore Eag1 is commonly found in different tumours and tumour cell lines but not in the corresponding healthy tissues.

In previous reports, expression of Eag1 was analysed on two levels: on the transcriptional (mRNA) level, using techniques such as northern blotting, RT-PCR or in situ hybridisation, and on functional level, on the basis of electrophysiological data.

\subsubsection{Distribution of the Eag1 protein in rat organs}

The techniques mentioned above gave a good indication about the functional expression of Eag1 in different cell-types, but the tissue-specific protein pattern, processing or posttranslational modifications remained to be analysed. First, the generation of specific antibodies was necessary to approach this topic by western blotting.

During the Ph.D. work, several antibodies were purchased by our group. Among them the 2413x polyclonal antibody directed against the C-terminus of rat Eag1 was chosen for western-blotting. This antibody also recognises the C-terminus of human Eag1. Additionally, the $2413 \mathrm{x}$ antibody cross-reacts with Eag2, but only when used in high concentrations. More recently, two monoclonal antibodies directed against the pore domain (mAb-62) and the C-terminus (mAb-33) of hEag1 were also obtained. These antibodies recognise the native tetramer structure of Eag1 channels, and thus were very useful for immunoprecipitation and cytochemistry, but not for western blotting. 
To complement the already mentioned results, we investigated the expression pattern of Eag1 at the protein level by western-blotting experiments with the $2413 x$ polyclonal antibody using rat tissues.

Membrane-enriched fractions were isolated from homogenised organs (brain, testis, muscle, kidney, lung, liver, eye, tongue, heart, spleen and epididymis) dissected from a 10-week old male. Samples were separated by SDS-PAGE (4-15\% continuous-gradient) followed by western blotting with the $2413 x$ polyclonal antibody.

As shown in Figure $8 \mathrm{~A}$, the clearest signal was observed, as expected, in brain. A strong broad band with an apparent molecular mass of approximately 120-140 kDa was observed, which differed slightly from the predicted molecular mass of rat Eag1 (SWISS-PROT, Q63472: 108,291 kDa). Moreover, a weak diffuse signal at ca. $250 \mathrm{kDa}$ as well as a clear band migrating at $>450 \mathrm{kDa}$ were found in the brain lane. Since these signals have about two and four times higher molecular masses than the predicted Eag1 band, we believe that they represent non-denatured di- and tetramers of the Eag1 protein respectively, although the possibility that they represent Eag1-containing complexes cannot be excluded.

A signal of similar size like in brain was detectable in eye, although the band was not so diffuse and migrated at approximately 120-130 kDa (Fig. 8 B). Since expression of Eag1 transcript in bovine retina has been previously reported (Frings et al., 1998), it is likely that this band corresponds to Eag1 protein expressed in rat eye tissue. Please note, that on membrane A $150 \mu \mathrm{g}$ and on membrane B $70 \mu \mathrm{g}$ of proteins were analysed. Although the exposure time of the membrane B was adequately prolonged for compensation, a direct comparison of signal intensities is not possible.

In rat testis, $2413 x$ polyclonal antibody recognised a clear band with an estimated molecular weight of $c a .60 \mathrm{kDa}$, but it was much smaller than the major band observed in brain. This band could represent testis specific truncated isoform of Eag1 in rat, since Eag1 expression in human testis was observed by real-time PCR (Pardo, unpublished data), but a cross-reactivity of the antibody cannot be excluded. The different size of Eag1 in rat testis could be caused by a testis-specific splice variant of Eag1 mRNA or a proteolytic posttranslational modification of the fully synthesised protein.

Furthermore, there was a very faint band detectable in lung $(55 \mathrm{kDa})$ as well as a band of about $50-55 \mathrm{kDa}$ observed in almost all tissues tested, but the specificity of these signals is questionable.

Placenta, previously reported to express Eag1 mRNA, was not tested in this experiment. 


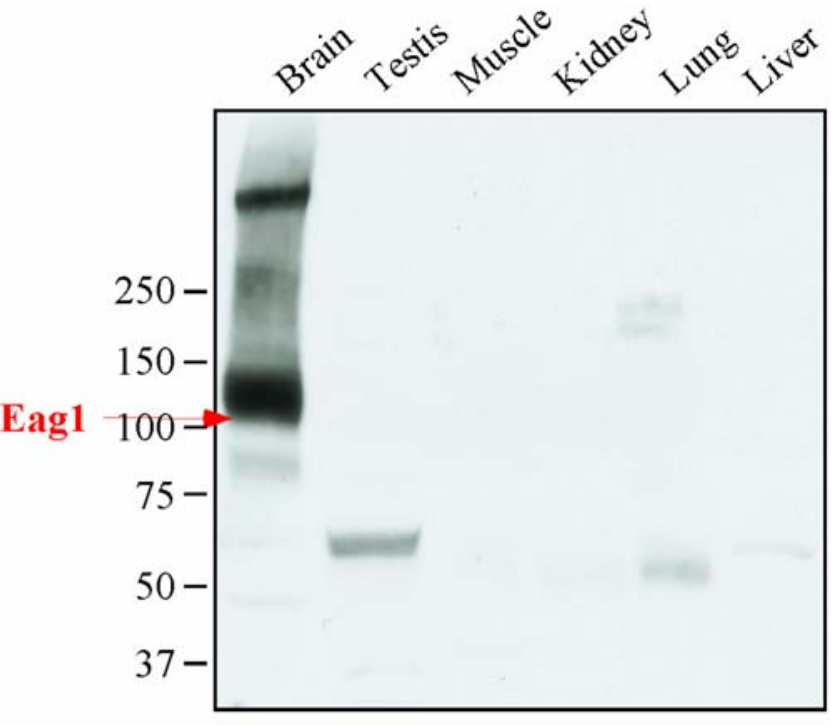

A

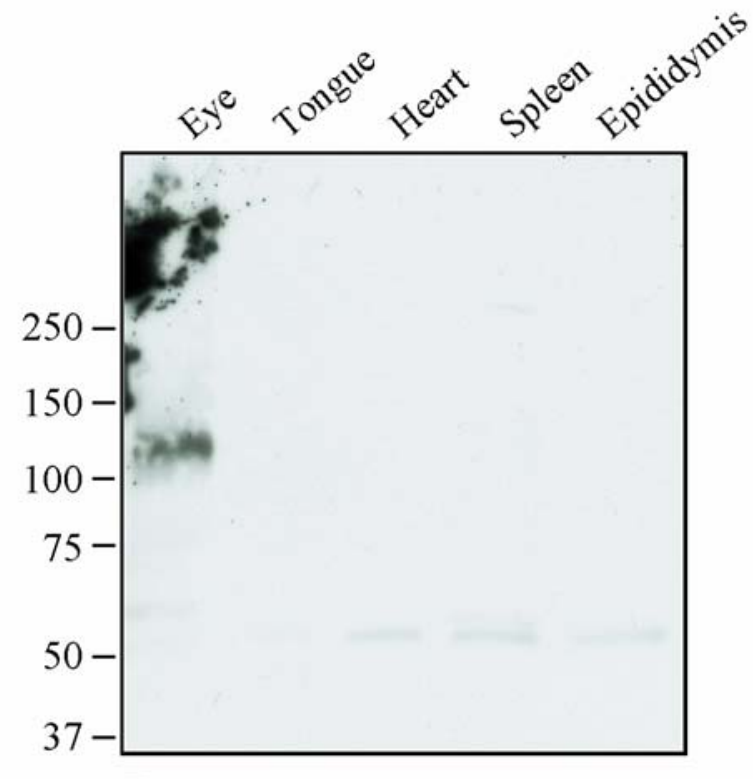

B

Fig. 8 Expression pattern of Eag1 protein in rat tissues

Western blot analysis of $150 \mu \mathrm{g}$ per lane of membrane-bound protein fractions isolated from rat brain, testis, muscle, kidney, lung and liver (A), and $70 \mu \mathrm{g}$ per lane of membrane-bound protein fractions from eye, tongue, heart, spleen and epididymis (B). Proteins were separated on 4-15\% continuous-gradient SDS-PAGE gels and immunodetected with the $2413 \mathrm{x}$ polyclonal antibody. Note, membrane B was exposed about twice as long to compensate differences in protein level.

Numbers on the left side correspond to molecular weights represented by marker proteins (in $\mathrm{kDa}$ ). Red arrow indicates the expected position of the Eag1 band (108 kDa, based on calculated molecular weight for the rat Eag1 protein).

The anti-Eag1 antibody detected a strong broad band of 120-140 kDa in brain and a band of 120-130 kDa in eye, corresponding to a monomer of the Eag1 channel molecule. Additional signals at 240-280 and $>400 \mathrm{kDa}$ are recognisable in brain that may correspond to nondenatured di- and tetramers. A clear band, migrating at about 60 $\mathrm{kDa}$, is detectable in testis but the origin of this signal was not further analysed.

\subsubsection{Immunoprecipitation of the Eag1 protein from brain homogenates}

In western-blot experiments, the main signal detectable in rat brain differed slightly from the calculated molecular mass of rat Eag1 (120-140 kDa instead of $108 \mathrm{kDa})$. To characterise the molecular weight of this brain-specific signal in detail, immunoprecipitation experiments, increasing the specificity of Eag1 detection, were carried out from rat brain tissues. Parallel to the immunoprecipitates, $25 \%$ of the brain homogenate used for IP was separated on the same gel.

As shown in Fig. 9 A, western-blot analysis of the immunoprecipitates from brain homogenates (membrane fractions), led to the detection of specific signals only when precipitated with anti-Eag1 antibody (protein-A-coupled 2413x polyclonal antibody; Eag1 IP). 
In control precipitations performed with a non-relevant antibody (protein-A-coupled anti-AMPA), as well as with Protein-A alone, no corresponding signals were detectable.

Surprisingly, instead of the intensive single band of about 120-140 kDa detected in the tissuewestern blot experiment (Fig. 8), two clearly distinguishable bands of approximately 115 and $130 \mathrm{kDa}$ were found in both IP and brain homogenates (Fig. 9). The signals were comparable in both cases: the intensity of the $115 \mathrm{kDa}$ signal was slightly higher then the intensity of the $130 \mathrm{kDa}$ signal. We will refer to these bands as E-115 and E-130, respectively (E stands for Eag and the number indicates apparent molecular masses).

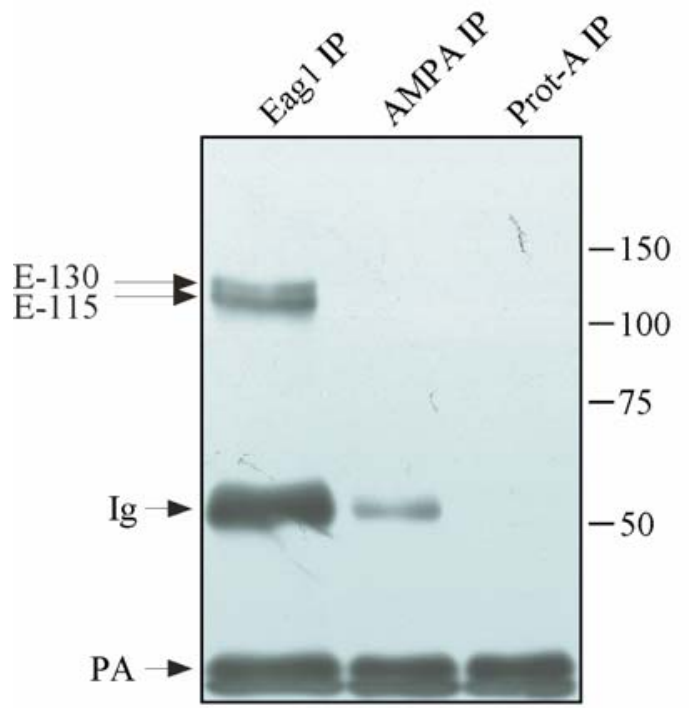

A

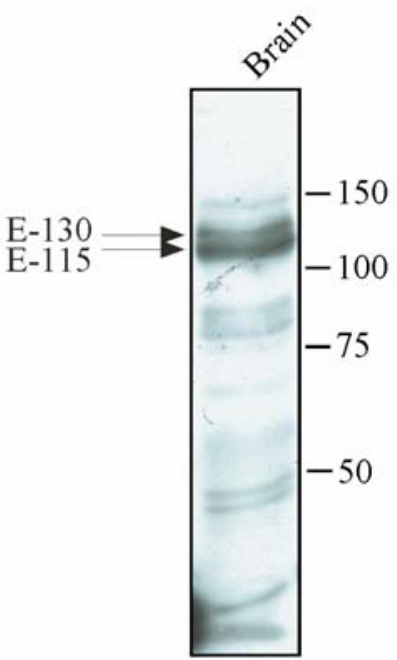

B

Fig. 9 Expression pattern of the Eag1 protein in rat brain

Membrane fractions were isolated from rat brain homogenates and proteins were either immunoprecipitated or directly separated on 7,5\% SDS-PAGE followed by western blotting. Eag1 signals were detected with $2413 \mathrm{x}$ polyclonal antibody.

A) Immunoprecipitation from brain homogenates performed using:
- 2413x polyclonal antibody
(Eag1 IP),
- $\quad$ anti AMPA polyclonal antibody
(AMPA IP)
- Protein A-Agarose alone
(Prot-A IP)

B) Control lane containing membrane proteins from rat brain homogenates

Positions of two bands (E-115 and E-130), corresponding to Eag1 isoforms are indicated by red arrows. Additional signals presumably represent the antibody (Ig) and Protein A (PA) are marked with black arrows.

In brain homogenates several additional bands at lower molecular weights, as well as a faint signal slightly higher than E-130 were detectable. Although all homogenisation and purification steps were performed on ice in the presence of protease inhibitors, we suggest proteolytic degradation as a cause of these bands, since corresponding signals were not detectable in other experiments. The band at $c a .150 \mathrm{kDa}$ could represent an Eag1-containing protein-complex, although unspecific binding of the antibody is another plausible explanation. 
The bands at 50 and $37 \mathrm{kDa}$ found in the immunoprecipitation presumably represent IgG and Protein A, respectively. The strong and weak Ig signals in Eag1 and AMPA lanes are probably due to recognition by the secondary antibody. Although equal amounts were used for IP, differences in binding can be assumed. Re-probing of the membrane with a specific AMPA antibody yielded the expected AMPA signal in the appropriate immunoprecipitate (data not shown).

Summarising, in immunoprecipitations of Eag1 from homogenates of rat brain membranes two specific bands, E-115 and E-130, appeared on the western blot, which were absent in control reactions. The divergence in the signal detection between the tissue-western blots (Fig. 8) and the immunoprecipitation-western blots (Fig. 9) can be explained by differences in resolution of polyacrylamide gels (7,5\% instead of $4-15 \%$ continuous gradient SDS-PAGE) as well as by differences in total intensity of the observed signals.

The immunoprecipitation experiments show, that two populations of the Eag1 protein are detectable in rat brain, and that both molecular masses seem to be higher than the predicted one.

\subsubsection{Glycosylation of the Eag1 in rat brain}

The calculated molecular mass of the unprocessed rat Eag1 is approximately $108 \mathrm{kDa}$. In contrast, in rat brain homogenates and immunoprecipitates, two distinguishable Eag1 isoforms were detected, with molecular masses estimated to approximately 115 and $130 \mathrm{kDa}$ (Fig. 9). The differences between the calculated and detected molecular masses of the rat Eag1 protein, as well as the fact that brain Eag1 runs as a broad band in polyacrylamide gel (Fig. 8), indicate possible posttranslational modifications occurring on the Eag1 protein.

To test whether glycosylation, the most common modification of membrane proteins, can account for the discrepancies between calculated and apparent molecular weights, an enzymatic deglycosylation reaction was performed on the Eag1 protein immunoprecipitated from rat brain membranes. Peptide N-glycosidase $\mathrm{F}$ (PNGaseF) was chosen as endoglycosidase, since this enzyme is known to attack virtually all glycosydic bonds. 
Eag1 was immunoprecipitated from $500 \mu \mathrm{g}$ of rat brain homogenates (membrane-bound fraction) with $1 \mu \mathrm{g}$ of $2413 \mathrm{x}$ bound to Protein A/G-Beads, and treated with PNGaseF (as described in 2.2.3.5). As a control an identical sample was incubated in parallel with the reaction buffer. Samples were separated on 7,5\% SDS-PAGE followed by western-blotting and immunoblotted with $2413 x$ polyclonal antibody.

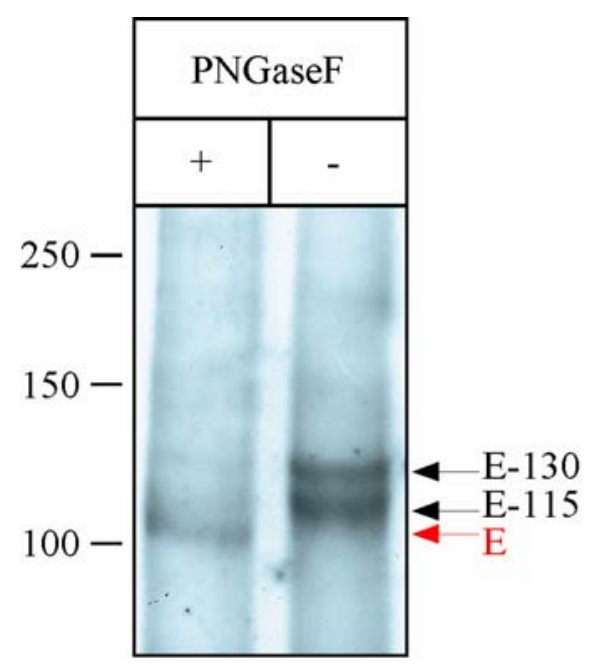

Fig. 10 Deglycosylation of Eag1 from rat brain

Western blot using polyclonal anti-Eag1 antibody after overnight treatment with (+) or without (-) PNGaseF. Positions of the E-115 and E-130 bands are indicated by black arrows. Note the single band at $\approx 110 \mathrm{kDa}$ in the treated sample (E, red arrow) corresponding to unglycosylated Eag1 protein.

As shown in Fig. 10, endoglycosidase treatment resulted in a shift of the apparent molecular mass of both Eag1 bands (E-130 and E-115), which migrated as a single band of about 110 $\mathrm{kDa}(+)$ after overnight incubation with PNGaseF. The control reactions exclude a contribution of intracellular proteases to the reduction in molecular weight, since $2413 \mathrm{x}$ antibody recognised both E-130 and E-115 bands in the control line. Moreover we didn't detect bands of lower molecular weights which would suggest proteolytic degradation.

Thus, these data strongly suggest that Eag1 protein undergoes N-linked glycosylation in rat brain. Two differently glycosylated species represented by E-130 and E-115 bands are coexisting in rat brain, in which oligosaccharides contribute with about $5 \mathrm{kDa}$ and $20 \mathrm{kDa}$ to the molecular mass of the protein. 


\subsection{Biochemical and functional characterisation of human Eag1 expressed in heterologous systems}

\subsubsection{Expression of hEag1 in CHO cells}

Since the preliminary aim of this study was to analyse posttranslational modifications of human Eag1 protein (hEag1), we further analysed the glycosylation of hEag1 expressed in heterologous systems. For that purpose Chinese hamster ovary $(\mathrm{CHO})$ cells were chosen as a model, since they do not express large amounts of endogenous potassium channels, thus simplifying the biochemical and electrophysiological analysis of the hEagl channel.

As source for all further experiments, a cDNA clone containing the complete open reading frame of hEag 1 corresponding to the splice variant "a" of hEag1 was used. This splice variant lacks 81 nucleotides corresponding to amino acids $318-344$. The used cDNA clone includes as well additional 36 nucleotides upstream from the ATG codon (5' UTR) and 78 nucleotides downstream from the stop codon (5' UTR). To simplify the nomenclature, this clone was named hEag1 (for the complete hEag1 sequence see NCBI: AJ001366).

hEag1 was cloned into the pTracer vector and stably transfected into CHO cells (Dac-30 method, see 2.2.4.6), giving rise to the CHOhEagl cell line. The pTracer vector carries a selection marker, a GFP-Zeocin fusion protein, under the control of an independent (SV40) promoter. For positive selection of transfectants, clones were selected in medium containing $100 \mu \mathrm{g} / \mathrm{ml}$ Zeocin, followed by permanent growth in Zeocin-complemented medium. Expression of hEag1 in CHO cells was tested by RT-PCR (data not shown) as well as by immunoprecipitation and western blotting (Fig. 11).

Consistent with the results from rat brain, polyclonal $2413 \mathrm{x}$ antibody detected two clearly distinguishable bands of approximately 110 and $130 \mathrm{kDa}$ (E-110 band and E-130 band) on western blots. These bands were visible in immunoprecipitations with polyclonal $2413 \mathrm{x}$ antibody (Fig. $11 \mathrm{~A}$ ), as well as with monoclonal mAb-62 antibody (Fig. $11 \mathrm{~B}$ ), although in the 2413x IP the intensity of E-130 was slightly lower than the intensity of E-110. 


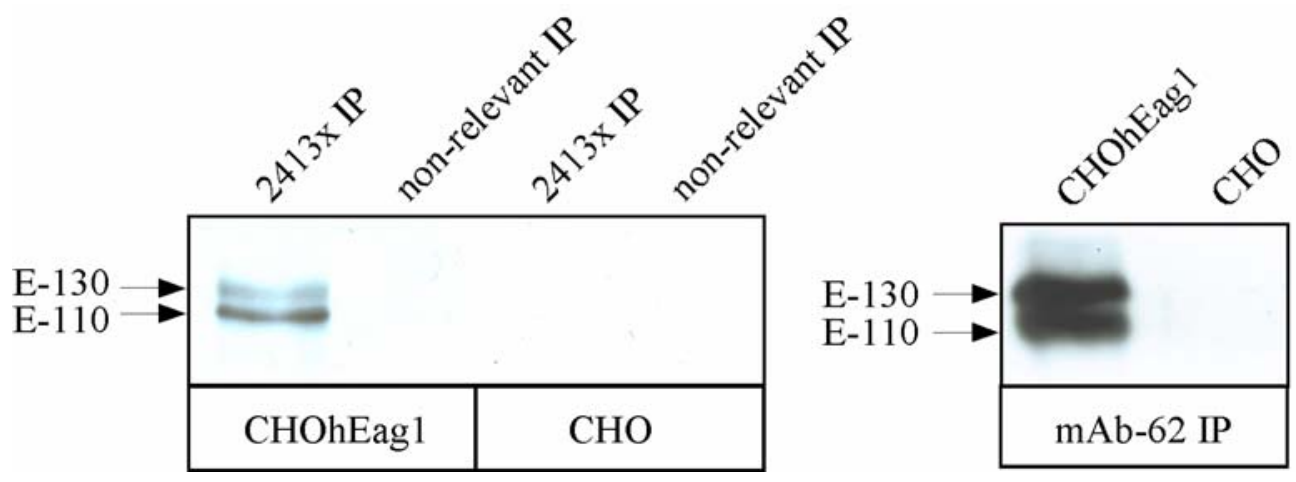

Fig. 11 Expression of hEag1 in CHO cells

CHO-hEag1 cells and nontransfected $\mathrm{CHO}$ cells were immunoprecipitated with anti-Eag1 antibodies followed by regular western-blotting and immunodetection with $2413 x$ polyclonal antibody. Two bands, with molecular weights estimated to 110 and $130 \mathrm{kDa}$ (E-110 and E-130), were detected only in CHOhEag1 cells, while in untransfected cells and anti-Lamin A/C precipitates no signal was detectable.

A) Immunoprecipitation with $2413 \mathrm{x}$ antibody and control precipitation with Lamin A/C antibody

B) Immunoprecipitation with mAb-62 antibody

Also here, molecular masses of the detected bands were slightly higher than the calculated molecular mass of hEag1 of 108595.83 Daltons. In the control precipitation with non-relevant antibody (anti-Lamin A/C) no adequate signal was observed. Similar, in untransfected CHO cells, 2413x polyclonal antibody did not recognised any signal neither in anti-Eag1 nor antiLamin A/C precipitates, which confirmed the specificity of the detected signal.

\subsubsection{Analysis of hEag1 sensitivity to endoglycosidases}

The two-band pattern of hEag1 expressed in CHO cells is very similar to the one obtained with rEag1 in brain homogenates. We therefore tested if hEag1 becomes glycosylated also during its maturation in $\mathrm{CHO}$ cells. To study glycosylation of hEag1 in detail, immunoprecipitated protein was treated with two different endoglycosidases: Endoglycosidase H (EndoH) and Peptide N-Glycosidase F (PNGaseF). Whereas the PNGaseF cleaves almost all known oligosaccharides from the protein, EndoH is able to act only on socalled ,core' glycoproteins, which are simple, mannose-rich, and usually an endoplasmaticreticulum residual form of glycoproteins (see Introduction 1.3.1 and Fig. 12 D). 
hEag1 was immunoprecipitated from total CHOhEag1 cell lysates using mAb-62 antibody. The protein was subsequently released from the Agarose-beads by boiling with SDS/methanol solution $(0,1 \%$ each) and incubated overnight with $5 \mathrm{U}$ of either EndoH or PNGaseF. Changes in mobility of hEag 1 were analysed on 7,5\% SDS-PAGE gels. Additionally, control reactions with all components except the enzyme were performed to exclude contribution of cellular proteases to migration shifts.

Treatment with PNGaseF (Fig. 12 A) affected the electrophoretic migration of hEag1, similar to the one observed in rat brain. After PNGaseF digestion, a single band of $\approx 108 \mathrm{kDa}$ was detected (Fig. 12 A, PNGaseF, +). The double-band pattern of native hEag1 was not affected in control reactions (Fig. 12 A, PNGaseF, -) which strongly suggests that the observed migration shift was not due to the action of intracellular proteases.

These data strongly support the idea that human Eag1 protein, similar to rat brain Eag1, undergoes $\mathrm{N}$-linked glycosylation when expressed in $\mathrm{CHO}$ cells. Analogous to the observation in rat brain homogenates, there is a strong indication that two glycosylation isoforms, contributing with 2-5 and $20-25 \mathrm{kDa}$ to the molecular masses of hEag1 coexist in the $\mathrm{CHO}$ expression system.

In contrast, treatment of hEag1 with EndoH, gave rise to a double-band pattern, that differed from the one typical for the native hEag1 protein. After incubation with EndoH the lower band was migrating at about $108 \mathrm{kDa}$, rather than $110 \mathrm{kDa}$ observed in the control (Fig $12 \mathrm{~B}$, EndoH + ). Since EndoH acts only on so-called 'core-oligosaccharides' of proteins, this indicates that the E-110 band corresponds to a core-glycosylated, mannose-rich hEag1 glycoprotein.

Migration of the E-130 band seems not to be significantly influenced by EndoH treatment, although a detection of slight shift $(\approx 2 \mathrm{kDa})$, might be contradictory. Because of the resolution limit of the SDS-PAGE, the possible difference in E-130 migration before and after EndoH treatment remained unclear. Apart from this, no conversion in apparent molecular weight of E-130 to the size of unglycosylated protein was observed after EndoH treatment, suggesting that E-130 represents a complex-glycosylated (and thus EndoH resistant), most probably post-reticular form of the hEagl protein.

The shifts are not likely to be due to a time dependent modification (i.g. degradation) of the Eag1 protein due to the preparation, since in the control incubations an identical band pattern, like in the IP lane was observed. 


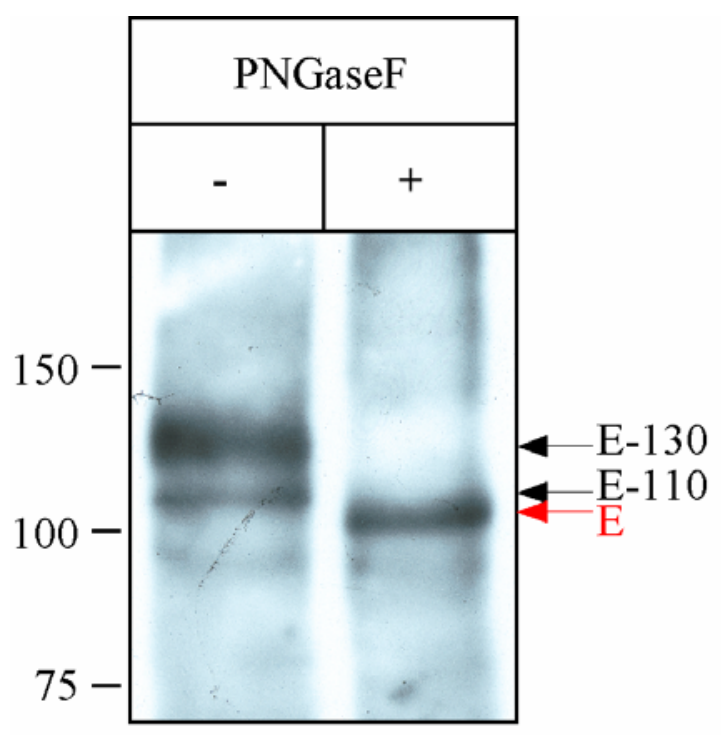

A

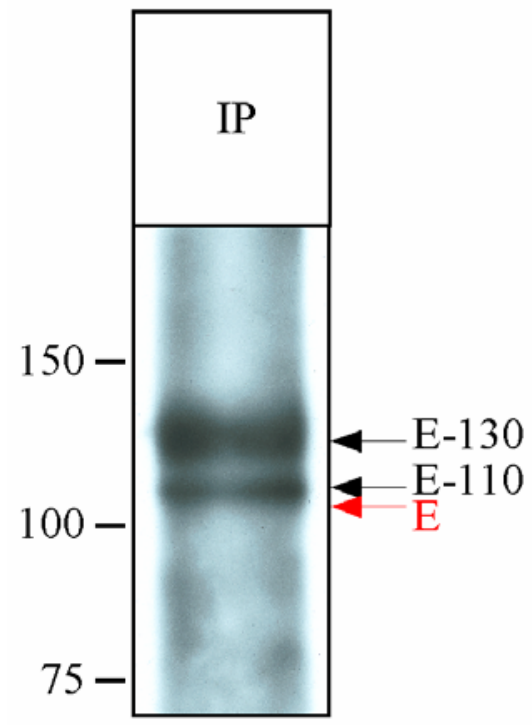

C

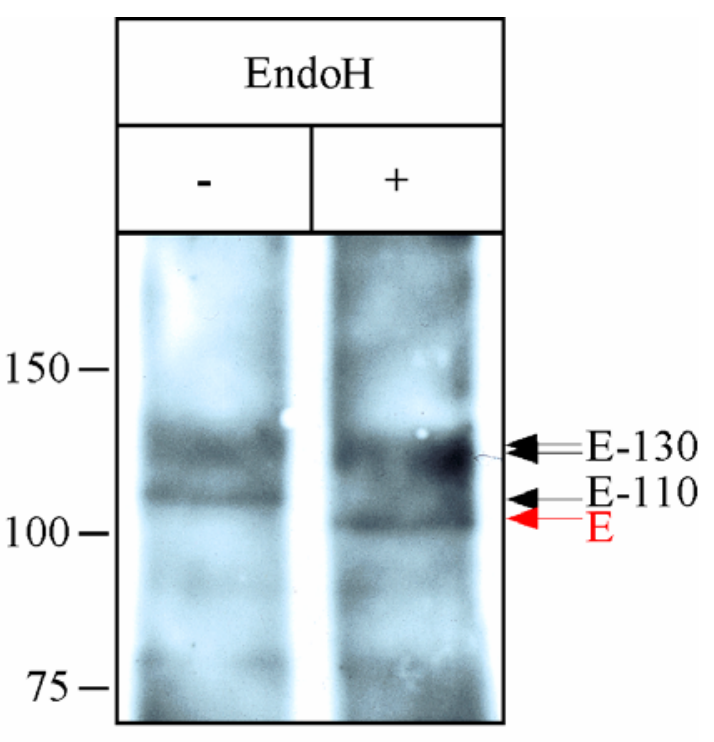

B
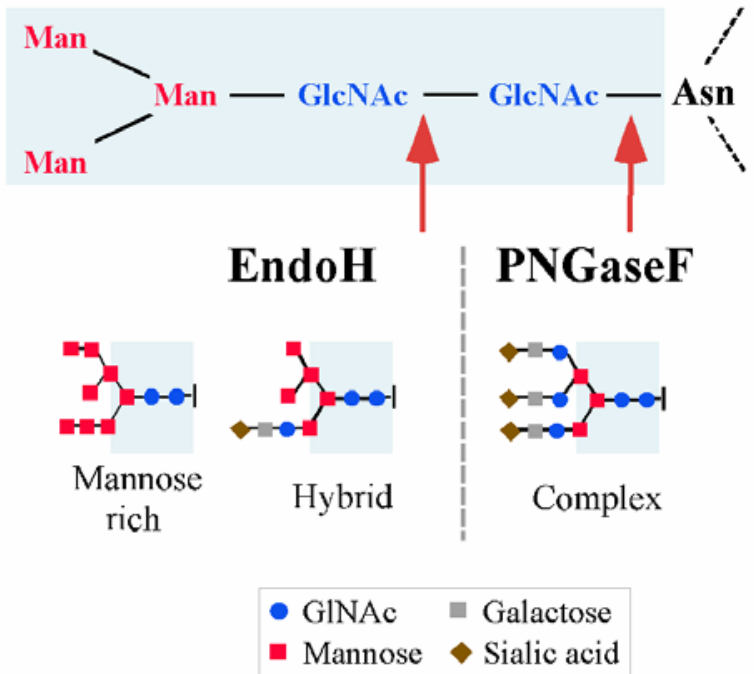

D

Fig. 12 Endoglycosidase treatment of hEag1 expressed in CHO cells.

CHOhEag1 cells were grown to confluence in $150 \mathrm{~cm}^{2}$ culture flasks, scraped, lysed, and the hEag1 protein was immunoprecipitated with $\mathrm{mAb}-62$ antibody from total cell lysates. Immunoprecipitates were treated overnight with PNGaseF (A) or EndoH (B) followed by regular western-blotting.

Positions of E-130 and E-110 bands are indicated by black arrows, position of the unglycosylated hEag1 by red arrows (E)

- $\quad$ hEag1 after incubation with (+) and without (-) PNGaseF.

A single band migrating at the level of predicted molecular mass of hEag1 $(\approx 108 \mathrm{kDa})$ is detectable after PNGaseF treatment.

- $\quad$ hEag1 after treatment with (+) and without (-) EndoH.

A shift in migration of the E-110 band to $\approx 108 \mathrm{kDa}$ is observed after EndoH treatment. Note the putative slight shift in migration of E-130 band

- non-treated immunoprecipitate.

- $\quad$ schematic representation of endoglycosidase specificity.

Endo $\mathrm{H}$ recognises only mannose rich and hybrid type of oligosaccharide attachments, PNGase F recognises also complex-glycosylated proteins 
These data indicate the existence of two distinct glycosylation isoforms of the hEag1 protein:

- E-110, which is sensitive to both, PNGaseF and EndoH, and thus is likely to represent a mannose-rich, core-glycosylated Eag1 protein

- E-130, which is sensitive only to PNGaseF but insensitive to EndoH and is therefore representing a complex-glycosylated form of the hEag1 protein.

The putative slight shift in the E-130 band by EndoH treatment could be caused by an additional core-glycosylation present on a distinct residue on the complex-glycosylated hEag1 protein.

\subsubsection{Disruption of the hypothetical glycosylation sites by site directed mutagenesis}

Considering the increasing amount of experimental data, highlighting the importance of $\mathrm{N}$-glycosylation for proper function of membrane proteins, including ion channels (see introduction), we decided to study the biochemical and functional effects of glycosylation of hEag1 in more detail.

As already mentioned, all known N-linked oligosaccharides are attached to the side chain of an asparagine in the consensus sequence Asn-Xaa-Ser/Thr (where Xaa can be any amino acid except Pro and Asp). Using computer-based comparative analysis (ScanProsite at ExPASy), six putative N-linked glycosylation sites could be identified in the hEag1 sequence (Fig13). The six putative glycosylation sites are asparagines $(\mathrm{N})$ located at positions: 26, 238, 388, 406, 470 and 752. Following an hypothetical structural model, based on the commonly accepted model of 6-transmembrane-domain channel, four of the putative glycosylation sites would be positioned in the predicted intracellular domains (26, N-terminus and 752 , C-terminus) or transmembrane regions (238, in S1 and 470, in S6), whereas two of them (388 and 406) would be located in the extracellular loop between the S5 domain and the P (pore) region of hEag1 (Fig. $13 \mathrm{~A}$ and B).

All six predicted N-glycosylation sites (N26, N238, N388, N406, N470 and N752) were mutated as described in 2.2.2.10.3. Site-directed mutagenesis was carried out to substitute glycosylatable asparagine residues with glutamine residues (Fig. 13). The hEag1-pTracer construct served as a template for all six mutant plasmids. Exact sequences of the oligonucleotides used in the PCR reaction and the mutation they introduced are listed 
in the Materials and Methods part (Table 1; 2.2.2.10.3). All created mutants were entirely sequenced to exclude any additional mutations in the hEag1 sequence. Nucleic acid replacement was followed by stable expression of the hEag1 mutants in $\mathrm{CHO}$ cells and their biochemical and functional analysis. To minimise effects of the specific integration site, polyclonal cell lines were used in all further studies, under the assumption that cells carrying the plasmid in several different integration sites were present in the preparation.
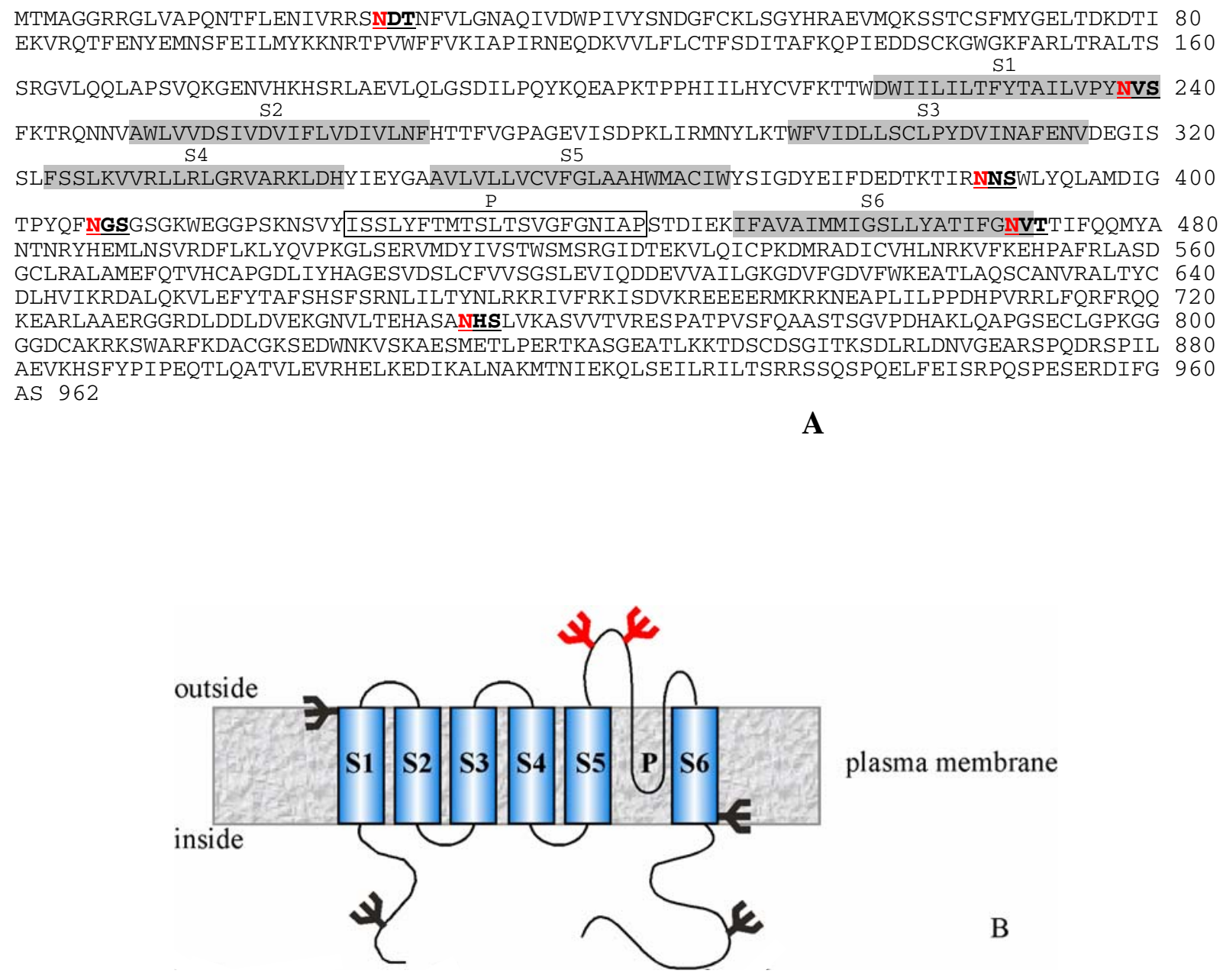

Fig. 13 Schematic domain organisation and putative N-glycosylation sites of hEag1

A) Sequence of the hEag1 protein.

The six predicted N-linked glycosylation motifs are bold and underlined. Putatively glycosylation sites are presented in red. Grey bars correspond to the presumed transmembrane regions S1-S6; empty (white) bar represents the putative $\mathrm{P}$ region of the hEagl channel.

B) Schematic representation of the positions of putative glycosylation sites on the structure model of the hEag1 monomer. Blue cylinders represent six transmembrane domains (S1-S6).

Putatively extracellular glycosylation sites are depicted in red, intracellular or transmembrane sites in black. 


\subsubsection{Selection of the mutant phenotypes with enzymatic deglycosylation}

To identify asparagine residue(s) participating in attachment of carbohydrate chains to hEag1, glycosylation of the generated hEag1 mutants (hEag1-N26Q, -N238Q, -N388Q, -N406Q, -N470Q and -N752Q) was examined with endoglycosidase (PNGaseF). For this, stably transfected cells expressing the appropriate mutants were lysed, and $9500 \mu \mathrm{g}$ of total cellular proteins were immunoprecipitated with $\mathrm{mAb}-62$-Protein A conjugated beads. The precipitates were aliquoted and treated overnight either with (+) or without (-) PNGaseF followed by separation on 7,5\% SDS-PAGE and regular western-blotting with $2413 x$ polyclonal antibody. Comparison of the control samples (Fig. 14) revealed that the migration pattern of the hEag1-N26Q, -N238Q, -N470Q and -N752Q mutants was not affected by the mutation, showing typical 2-band pattern (110 and $130 \mathrm{kDa}$ ) observed for hEag1. Endoglycosidase treatment of the four mutant proteins resulted in the conversion of both bands to a single one, migrating at $\approx 108 \mathrm{kDa}$, as shown for hEag1 (see Fig. 12). The electrophoretic mobility pattern of these mutant proteins, identical with the one obtained for hEag1, indicate that those mutated asparagine residues (N26, N238, N470 and N752) are not glycosylated in CHO cells (Fig. 14). This was an expected result, since these putative glycosylation sites are located in the predicted intracellular or transmembrane regions of the hEag1 channel.

In contrast, the immunodetection pattern of hEag1-N388Q and -N406Q mutants was strongly influenced by the mutation.

Fig. 14 clearly shows that hEag1-N388Q lacks the E-110 band, whereas a single band compatible with E-130 is still present. Treatment of hEag1-N388Q with PNGaseF resulted in a shift of the E-130 band to lower molecular weights $(\approx 108 \mathrm{kDa})$, corresponding to unglycosylated hEag 1 protein. This suggests that this band is of the same origin as the E-130 band of nonmutated hEag1.

Moreover, in the hEag1-N406Q mutant a single band migrating at $110 \mathrm{kDa}$ was detectable and compatible with the E-110 band. This band was also sensitive to PNGaseF, since a slight shift in migration (to $\approx 108 \mathrm{kDa}$ ), similar to the hEag1 E-110 band, was observed after endoglycosidase treatment.

In conclusion, out of the six possible glycosylation sites predicted in the hEag1 sequence, only two sites (N388 and N406) are glycosylated. 
Two different carbohydrate attachments are identified for the hEag1 protein.

- The complex-isoform attached to asparagine in position 406 is EndoH insensitive and corresponds to the $130 \mathrm{kDa}$ band on western blots.

- The core-isoform attached to asparagine 388 is EndoH-sensitive and corresponds to the $110 \mathrm{kDa}$ band on western blots.

\begin{tabular}{|l|l|l|l|l|l|l|l|}
\hline \multicolumn{2}{|c|}{ N26Q } & \multicolumn{2}{|c|}{ N238Q } & \multicolumn{2}{c|}{ N388Q N406Q } \\
\hline- & + & - & + & - & + & + & + \\
\hline & & & & & & & \\
& & & & & & & \\
\end{tabular}

\begin{tabular}{|l|l|l|l|l|l|}
\hline \multicolumn{2}{|c|}{ N470Q } & \multicolumn{2}{|c|}{ N753Q } & \multicolumn{2}{c|}{ CHO } \\
\hline- & + & - & + & - & + \\
\hline & & & \\
& & & \\
\hline
\end{tabular}

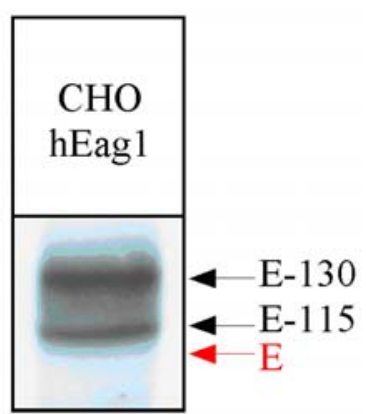

Fig. 14 Deglycosylation of N-linked carbohydrates in hEag1 mutants

Mutant proteins (hEag1-N26Q, -N238Q, -N388Q, -N406Q, -N470Q and -N752Q) were immunoprecipitated overnight from $\mathrm{CHO}$ cell-lysates with $\mathrm{mAb}-62$ antibody. Precipitates were divided into two equal samples and treated $\mathrm{ON}$ with $(+)$ or without (-) PNGaseF as described in 2.2.3.5.1, followed by regular western-blotting and immunodetection with the $2413 x$ polyclonal antibody. Arrows indicate the positions of the wild-type hEag1 bands that absent in the respective mutants.

\subsubsection{Generation and biochemical analysis of the double glycosylation mutant}

Since two asparagine residues on hEag 1 were identified to be differentially and independently $\mathrm{N}$-glycosylated, a double mutant lacking both glycosylation sites was generated and analysed. For this, the hEag1-N406Q-pTracer construct was used to introduce the second (N388Q) 
mutation using the conditions described above. The generated mutant (hEag1-N388,406Q) was entirely sequenced and stably expressed in CHO cells as described for other mutants.

As shown in Fig. 15 A, after the PNGaseF treatment of the double mutant, performed under previously applied standard conditions (overnight at $37^{\circ} \mathrm{C}$, without protease inhibitors), no band corresponding to the unglycosylated hEag1 was detected with $2413 \mathrm{x}$ antibody. Similarly, no band corresponding to hEagl was detected in the control reaction (immunoprecipitated N388,406Q mutant protein incubated under the same conditions in reaction buffer without endoglycosidase), although a protein of appropriate size $(\approx 108 \mathrm{kDa})$ was found in the untreated immunoprecipitates (Fig. 15 B). However, in both cases bands migrating at less than $75 \mathrm{kDa}$ were detectable.

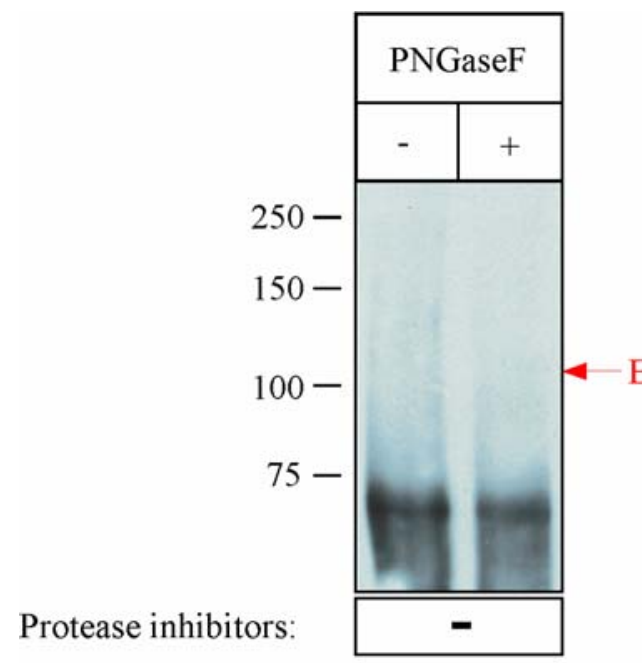

A

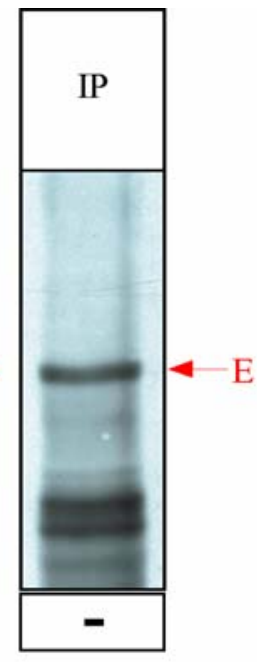

B

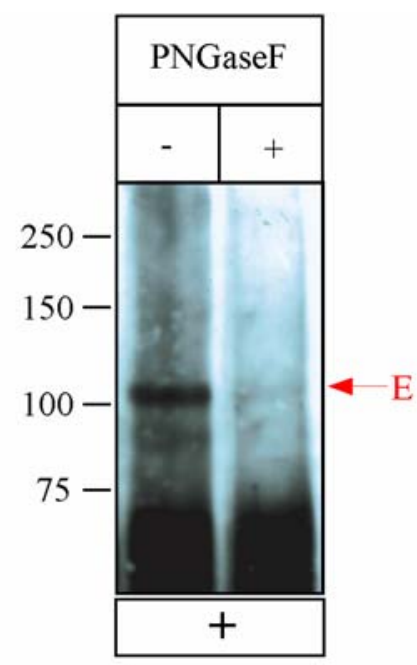

C

Fig. 15 Endoglycosidase treatment of N388,406Q double mutant

A) PNGase $\mathrm{F}$ treatment performed in standard conditions (overnight at $37^{\circ} \mathrm{C}$ without protease inhibitors)

B) control immunoprecipitation

C) PNGase $\mathrm{F}$ treatment in protease-reduced condition $\left(5 \mathrm{~h}\right.$ at $37^{\circ} \mathrm{C}$ with protease inhibitors)

In both cases degradation of the unglycosylated hEag1 was observed after incubation with PNGase F. In the control precipitation (B) a band of the expected size is observed.

This result suggested a proteolytic degradation of the hEag1-N388,406Q protein. During the immunoprecipitation, only a slight degradation was observed, which can be explained by the incubation at $4^{\circ} \mathrm{C}$. But, since the PNGaseF (or control) treatment was performed at physiological temperature $\left(37^{\circ} \mathrm{C}\right)$ the complete degradation of the protein must have occurred. To avoid proteolytic degradation, PNGaseF treatment in presence of protease inhibitors, and with reduced duration (5 hours) was performed. Fig. $15 \mathrm{C}$ shows, that under these conditions, a signal of the appropriate size $(\approx 108 \mathrm{kDa})$ was detectable in the control reaction (identical 
treatment except the enzyme). In contrast, in the enzyme treated sample, only a very faint band was visible. Additionally also in the presence of protease inhibitors a strong, diffuse signal lower than $75 \mathrm{kDa}$ was detectable. These data suggest, that besides proteases some other factors, can contribute to the degradation of hEag1-N388,406Q. This is not surprising, since it is known that glycosylation increases the stability of many membrane proteins. This results confirm this since complete loss of glycosylation in N388,406Q mutant leads to destabilisation of the hEag1 protein. Thus it can postulated, that N-linked glycosylation increases the stability and/or protects the hEag1 protein from degradation.

\subsubsection{Model of the $\mathrm{N}$-linked glycosylation of hEag1 potassium channel}

Variability in the detection pattern was observed for the hEag1-N388Q mutant in different experiments. A single band of about $130 \mathrm{kDa}$ was detected in some tests, for example in the western-blot analysis performed to identify active glycosylation site (see Fig. 14, control reaction for the PNGaseF digest of the hEag1-N388Q mutant). In contrast, an additional band of molecular weight estimated to less than $110 \mathrm{kDa}$ was detectable in other experiments (shown in later part of this work). This additional fragment was interpreted to be the fully unglycosylated variant of the hEag1 protein.

To match the exact position of the detected bands between all glycosylation mutants, the membrane fraction isolated from appropriate cell lines (CHO and $\mathrm{CHO}$ expressing: hEag1, hEag1-N388Q, -N406Q and -N388,406Q) was analysed on western blots. For this, $50 \mu \mathrm{g}$ of the membrane-bound fraction of proteins (isolated as described in 2.2.3.9.1) were separated on $7,5 \%$ SDS-PAGE, transferred onto nitrocellulose membrane and immunodetected with the 2413x antibody.

In wild-type hEag1 transfected cells the $2413 \mathrm{x}$ antibody detected the already described two bands of 110 and $130 \mathrm{kDa}$. In hEag1-N406Q expressing cells a single band corresponding to the core-glycosylated protein was detectable. In the double mutant (hEag1-N388,406Q) a single band of approximately $108 \mathrm{kDa}$ corresponding to the unglycosylated $\mathrm{hEag} 1$ protein was observed. 
In the hEag1-N388Q mutant (lacking the site for core-oligosaccharide attachment), the reported complex glycosylated $130 \mathrm{kDa}$ band was detected, but an additional band of about $108 \mathrm{kDa}$ was observed. This fragment migrated at the same level as the band in the double (non-glycosylated) mutant, strongly suggesting that the non-glycosylated hEag1 was the source for the $\approx 108 \mathrm{kDa}$ band in the hEag1-N388Q mutant. The presence of the nonglycosylated form of hEag1 in CHOhEag1-N388Q transfected cells can be caused by an inefficient processing of the single glycosylation site on the N388Q mutant protein, probably due to the introduced mutation.

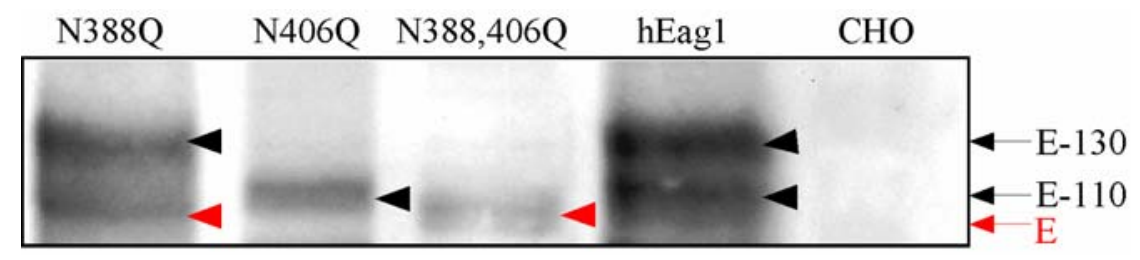

Fig. 16 Immunoblot analysis of the membrane fraction matching the hEag1 and N-linked glycosylation mutants of hEag1 stably expressed in $\mathrm{CHO}$ cells

$\mathrm{CHO}$ cells as well as CHO cells expressing wild-type hEag1 and mutant proteins hEag1-N388Q, -N406Q and $\mathrm{N} 388,406 \mathrm{Q}$ were collected and disrupted by osmotic pressure and membrane fractions were isolated as described in Materials and Methods (2.2.3.9.1, $1.000 \mathrm{x}$ g centrifugation step was directly followed by the $100.000 \mathrm{x} \mathrm{g}$ centrifugation in order to collect the total membrane bound protein pool). Following standard western-blotting, the membrane was probed with the $2413 x$ polyclonal antibody.

Black arrows indicate positions of the two glycosylated hEag1 forms (E-110 and E130), red arrows represent the position of unglycosylated hEag1.

Taken together, the following model of hEag1 glycosylation can be proposed (Fig. 17).

There are two putative hEag1-glycosylation isoforms:

- a core-glycosylated (EndoH sensitive) form corresponding to the E-110 band

- a complex-glycosylated (EndoH insensitive) form corresponding to the E-130 band.

Additionally, these two isoforms are related to two distinct glycosylation sites at asparagine 388 and asparagine 406:

- the Asn 388 is core-glycosylated with small sugar attached, since hEag1 detection pattern lacks the E-130 band when the 388 site is displaced

- in contrast the Asn 406 posses large and complex carbohydrate attachment, as mutation of this site eliminate E-130 band from the western blot. 

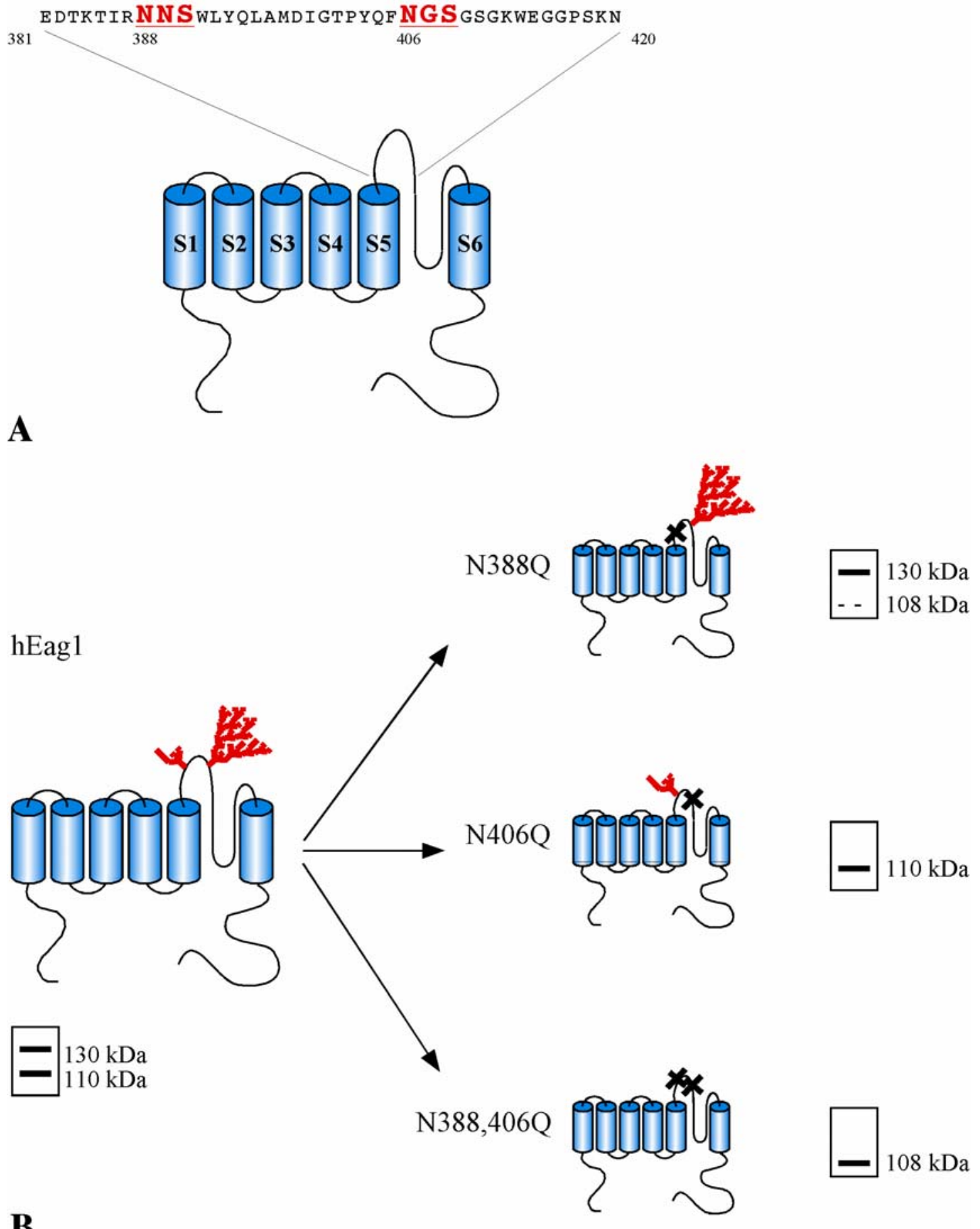

\section{B}

Fig. 17 Model of N-linked glycosylation of the hEag1 potassium channel

Schematic structure of a single hEag1 $\alpha$-subunit is presented. The six transmembrane domains (S1-S6) are shown as blue cylinders, while the P region is presented as a loop between S5 and S6.

A) Amino acid sequence harbouring the hEag1 glycosylation sites in relation to the region between S5 and S6 domains.

B) Schematic location of the two glycosylation isoforms of hEag1 and mutant proteins (N388Q,N406Q and N388,406Q).

The complex-glycosylated isoform is represented as a large oligosaccharide tree, whereas the coreglycosylated form is shown as a small sugar structure. Mutations of glycosylation sites are depicted as black crosses. For each model, a schematic depiction of the SDS-PAGE migration pattern is presented. In the N388Q mutant the dotted line represents the band corresponding to the unglycosylated protein (in some cases not detectable). 


\subsubsection{Binding of hEag1-linked oligosaccharides to Concanavalin A}

Lectins are proteins that interact with specific terminal sugar residues and are therefore useful probes for the study of cell surface carbohydrates (reviewed by Sharon and Lis, 1972). Concanavalin A (Con A) isolated from the jack bean (Canavalia envalin) is one of the most widely used and best-characterised lectins. Con A has broad applicability primarily because it selectively recognizes commonly occurring sugar structures $\alpha$-D-glucose and $\alpha$-D-mannose. Since a wide variety of the membrane glycoproteins have $\alpha$-linked glucose or mannose residues, these glycoproteins can be detected or purified using Con A.

In order to investigate the constitution and nature of the oligosaccharides attached to hEag1 and hEag1-mutant proteins expressed in CHO cells, affinity for Concanavalin A was tested. Cell lysates (3000 $\mu \mathrm{g}$ of each: CHO, CHOhEag1, CHOhEag1-N388Q and CHOhEag1-N406Q) were incubated overnight with $100 \mu \mathrm{l}$ of Concanavalin A-Agarose-beads. As a control, an immunoprecipitation reaction with $2 \mu \mathrm{g}$ of mAb-33/62 antibody mix was performed in parallel. Pellets were then separated on 7,5\% SDS-PAGE gels and probed with polyclonal antibody (2413x).

As shown in Figure $18 \mathrm{~A}$ in wild-type hEag1 both glycosylation isoforms, the $130 \mathrm{kDa}$ and the $110 \mathrm{kDa}$ bands, were detectable after precipitation with Concanavalin A. As expected, Con A also pulled down a single fragment of $110 \mathrm{kDa}$ from the N406Q mutant. In contrast, no band was detected after the precipitation with Concanavalin A from cells transfected with the N388Q mutant protein (Fig. 18 A). The lack of detection in the hEag1-N388Q mutant after immunoprecipitation with Con A was reproducible (data not shown).

Control immunoprecipitations reactions showed similar detection pattern as described, for example, in 4.2.6 confirming the presence of hEag1 and mutant proteins in the cell lysates (Fig. 18 B).

These results suggest that N388Q mutant does not contain oligosaccharides that can be recognised by Con $A$, i.e. $\alpha$-glucose or $\alpha$-D-mannose, which is not surprising in the case of complex-glycosylated proteins. In contrast, the N406Q mutant containing only coreoligosaccharides is well recognised by Concanavalin A.

Moreover, in wild-type hEag1 both bands, the $110 \mathrm{kDa}$ (core glycosylated) and the 130 (complex-glycosylated and unrecognisable by Con A in the N388Q mutant), are recognised by the lectin. The former binding is as expected, the latter recognition of the $130 \mathrm{kDa}$ band 
can be explained by double glycosylation on both sites, whereby the core-glycosylation site (N406) binds to Con A. Since the two bands are detected in CHO cells transfected with hEag1 and precipitated with Con A, both glycosylated isoforms exist together independently. A more detailed explanation is found in the discussion.
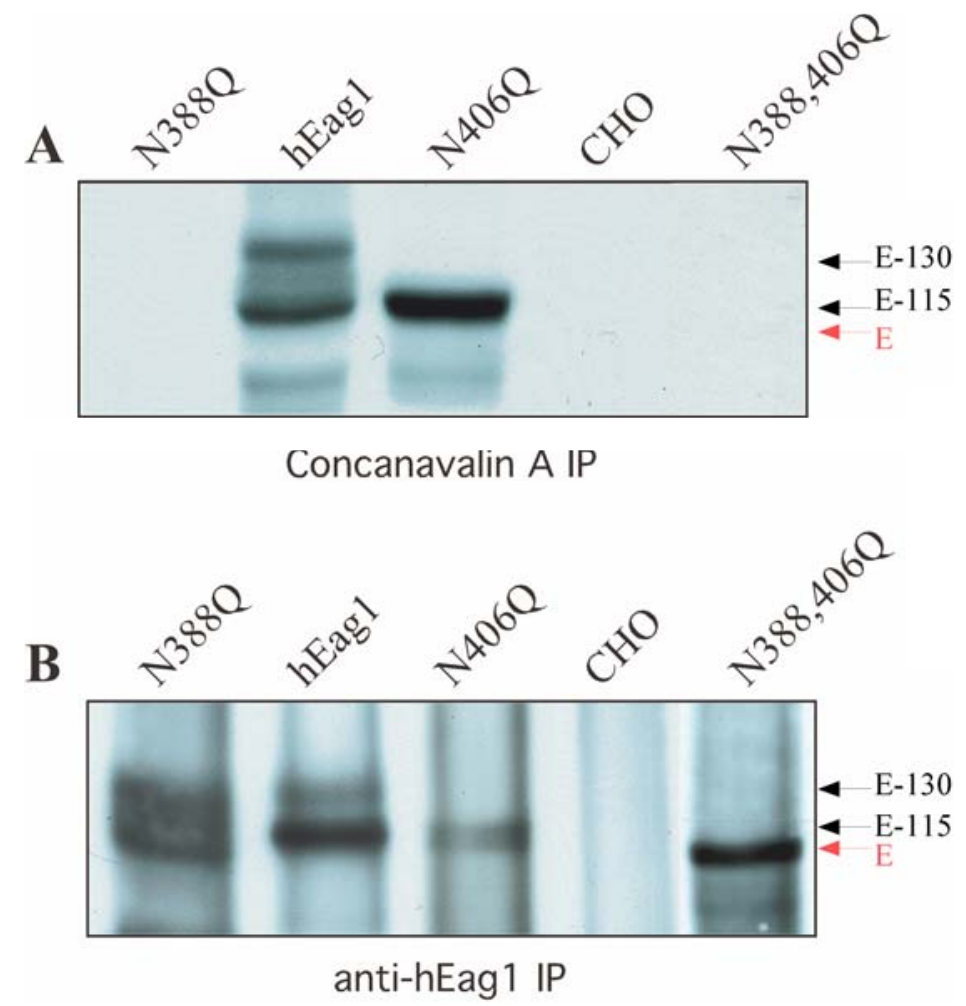

Fig. 18 Precipitation of hEag1 and mutant proteins with Concanavalin A

Proteins were precipitated over night from $3000 \mu \mathrm{g}$ of total cell lysates (CHO, CHOhEag1, CHOhEag1-N388Q and CHOhEag1-N406Q) with $100 \mu \mathrm{l}$ of Concanavalin A-Agarose (A) as well as with $2 \mu \mathrm{g}$ of mAb-33/62Protein A-Beads (B) as a control. After separation on 7,5\% SDS-PAGE and immunodetection with 2413x antibody, two bands corresponding to E-130 and E-110 were found in Con A precipitates from hEag1 transfected cells, as well as a single band $(\approx 110 \mathrm{kDa})$ in Con A precipitates from N406Q transfected cells. No signal was detected in N388Q mutant as well as in untransfected cells. In control immunoprecipitations the characteristic detection pattern is observed.

\subsubsection{Distribution of hEag1 glvcosvlation isoforms in subcellular fractions}

To test whether the two hEag1 glycosylation isoforms corresponding to the 110 and $130 \mathrm{kDa}$ bands on the western blot are distributed differentially in the cells, and whether the individual proteins might behave differently with respect to subcellular distribution, we examined distribution of hEag1 in the different cell fractions. 


\subsubsection{Fractionation by differential centrifugation}

To initially test the distribution of the hEag1 glycosylation isoforms in cell fractions, cells were fractionated by differential centrifugation, a method that allows a crude distinction between large complexes and cytosolic, nuclear or membrane-bound proteins.

CHO cells expressing hEag1 proteins were lysed and the subcellular fractions were separated in three centrifugation steps as described in 2.2.3.9.1. Pellets: P-1 (1.000 x g), P-10 (10.000 x g) and P-100 (100.000 x g) were collected, and proteins were separated on 7,5\% SDS PAGE gel, and detected by immunoblotting with $2413 \mathrm{x}$ antibody. As shown in Figure 19, hEag1 glycosylation isoforms were differentially found in the analysed fractions. Semi-quantitative comparison (by densitometry analysis) of the hEag1 arrangement through the three fractions is presented in Fig. 19 B. The density of the E-110 band was nearly the same in all three fractions, with slight increase in the P-10 fraction. In contrast, the intensity of the E-130 was dramatically increased, when comparing P-10 and P100 with P-1.

Analysis of the percentual contribution of each isoform to the total hEag1 signal in each fraction showed that in the P-1 fraction the E-130 represented majority (67 \%) of the hEag1 population. In contrast in P-10 and P-100 fractions the E-130 was the prevailing form, contributing with $69,7 \%$ and $79,1 \%$ to the detected signal respectively.

To monitor the fractionation, marker proteins were detected as well. GM-130, a Golgi residual protein, as well as TRAP $\alpha$, an ER integral membrane protein, fractionated as predicted mostly within the P-1 and P-10 fractions and both were only weakly represented in P-100 fraction, supposed to contain mainly plasma membrane sheets.

The intensity of the band corresponding to TRAP $\alpha$ was strong and similar in P-1 and P-10 fractions, whereas the signal of GM-130 was weaker, and best detectable in P-10 fraction. This fact suggests that both glycosylation isoforms of hEag1 are differentially distributed in cellular fractions. The E-110, thought to represent core-glycosylated protein, is mainly represented in P-1 fraction, in which a strong signal of ER-marker and medium signal of Golgi-marker were detectable. This is in agreement with the fact that core-glycosylated proteins are typical for ER and early Golgi.

The E-130 band represented the majority of hEag1 signal in the P-10 fraction (containing a majority of Golgi-marker and ER-marker), as well as in the P-110 fraction thought 
to contain a pool of plasma membrane proteins. Unfortunately, we were not successful in re-probing of the membrane with plasma-membrane marker thus the colocalisation of E-130 in plasma membrane enriched fraction remains to be explored.

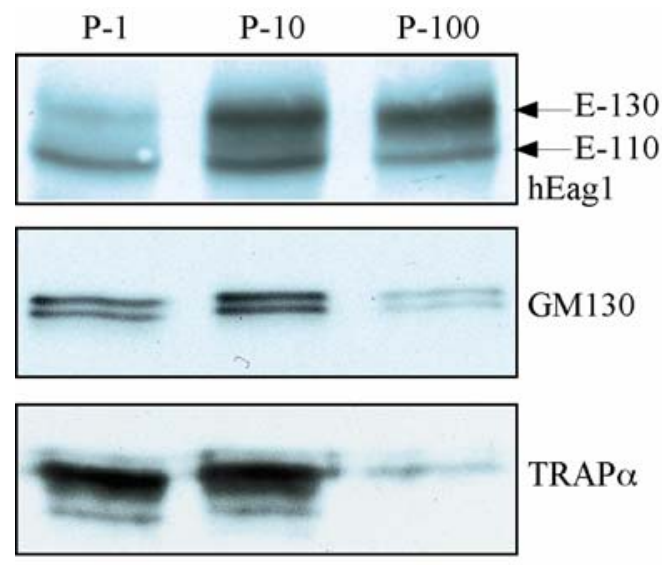

A

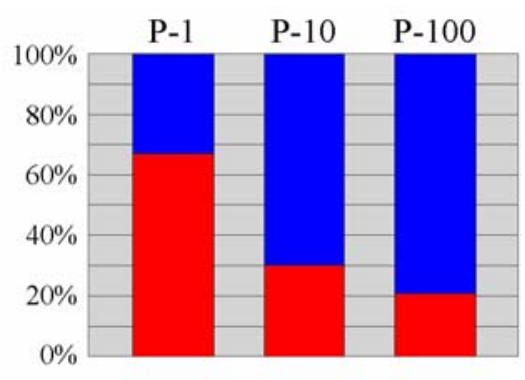

\begin{tabular}{|c|c|c|c|}
\hline & P-1 & P-10 & P-100 \\
\hline E-130 & $33 \%$ & $70 \%$ & $79 \%$ \\
\hline E-110 & $67 \%$ & $30 \%$ & $21 \%$ \\
\hline
\end{tabular}

B

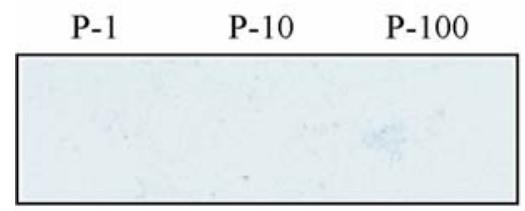

C

\section{Fig. 19 Cell fractionation by differential centrifugation}

A) hEag1 proteins from different fractions, P-1 (pellet after $1.000 \mathrm{x}$ g centrifugation), P- 10 (pellet after $10.000 \mathrm{x}$ g centrifugation) and P-100 (pellet after $100.000 \mathrm{x}$ g centrifugation) isolated from CHOhEag1 cells were analysed on western blot with antibodies against Eag1 protein (2413x), Golgi (anti-GM-130) and ER (anti-TRAP $\alpha$ ).

B) Densitometric analysis of the detected hEag1 signals. Diagram presents percentual contribution of the E-130 and E-110 to the total hEag1 signal in each fraction.

C) Parallel fractionation performed on untransfected CHO cells. Detection was performed with 2413x antiEag1 polyclonal antibody

\subsubsection{Fractionation by sucrose gradient}

Intracellular fractionation by differential centrifugation allowed only crude distinction between compartments such as nuclei, or "membrane" without clear differentiation of reticular or Golgi enriched fractions. Therefore the distribution of the hEag1 on sucrose gradients was examined. 12-60\% sucrose density gradients were prepared as described in 2.2.3.9.2. CHOhEag1 cells were lysed by osmotic pressure (efficiency was monitored under the microscope) and $500 \mu \mathrm{l}$ of the homogenate was loaded on the top of sucrose gradients and centrifuged for $3 \mathrm{~h}$ at $140-180.000 \mathrm{xg}\left(4^{\circ} \mathrm{C}\right.$, swinging bucket). 
Fig. 20 A shows the distribution of the hEag1 glycosylation isoforms on a $12-60 \%$ sucrose density gradient. Both hEag1 bands, E-110 and E-130 were detectable in the higher density fractions (between 8 and 17) with a maximum in fractions 14 and 15. In fractions 8-10 only very faint bands were detectable. The differences in the distribution of two glycosylation isoforms through the fractions were also observed, although they were not as dramatic as in the previous experiment. In fractions $8-11$ as well as $16-17$ no significant difference between the distribution of the two isoforms was observed. In contrast, in fractions 12-15 the E-110 band determined the majority of the detected signal. Additionally in fraction 14 and 15 the intensity of signal detected at $110 \mathrm{kDa}$ increased dramatically, whereas the signal at $130 \mathrm{kDa}$ showed only slight increase. Moreover an additional band of about $108 \mathrm{kDa}$, interpreted as unglycosylated hEag1 protein, was observed in fraction 13-15, showing strongest signal in fraction 14.
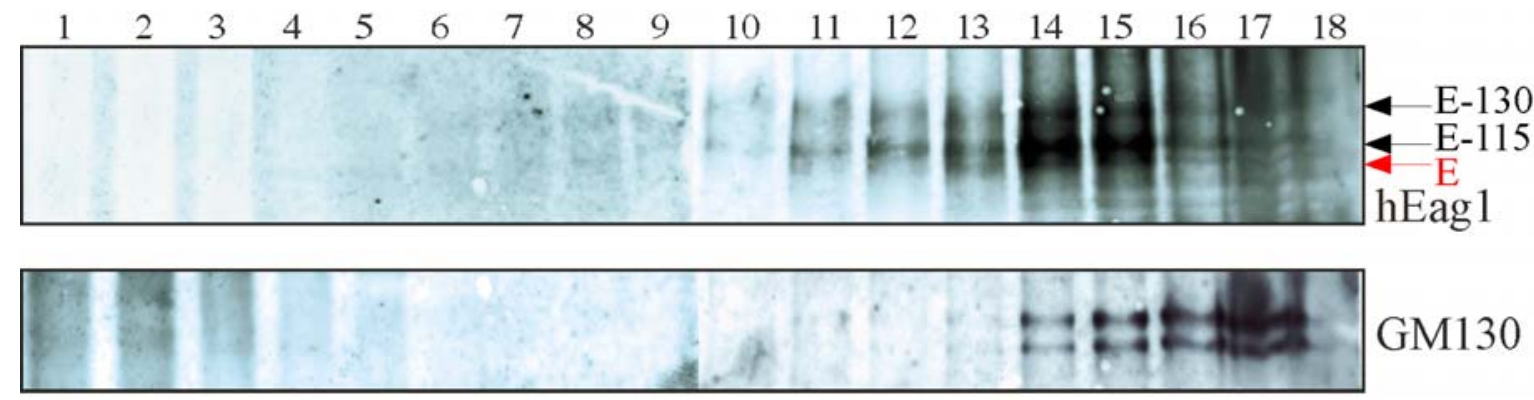

GM130

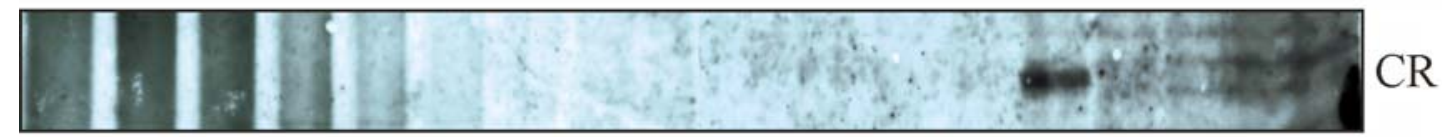

A

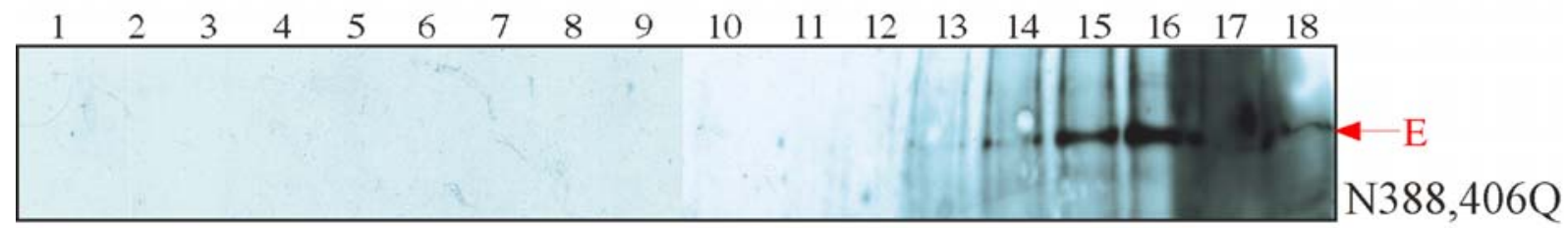

B

Fig. 20 Cell fractionation by 12-60\% sucrose gradient

CHOhEag1 (A) and CHOhEag1-N388,406Q (B) cells were lysed and fractionated on 12-60\% sucrose gradients. Fractions 1-18 were collected (from top to bottom) and 50 $\mathrm{fl}$ of each was separated on 7,5\% polyacrylamide gel (SDS-PAGE), followed by western blotting.

A) CHOhEag1; immunoblotting with:

- 2413x polyclonal antibody. Arrows indicate positions of E-130 and E-110 bands, as well as unglycosylated hEag1 protein (E)

- GM-130 antibody

- Calreticulin antibody (CR)

B) CHOhEag1-N388,406Q; immunoblotting with 2413x polyclonal antibody 
Distribution of the glycosylation deficient N388,406Q mutant on a 12-60\% sucrose density gradient is shown in the Figure $20 \mathrm{~B}$. The unglycosylated hEag1 protein localised as well to the high density fractions, but its distribution differed from the two glycosylated isoforms. Band of a proper size was detectable in fractions 12-18 with the maximum intensity in fraction 16, not in 14-15 as observed for the glycosylated proteins. In fractions 12-14 only a faint band was observed. Due to high background, no signal could be detected in fraction 17.

Both, Golgi and reticular proteins were also localised to the high-density fractions. Anti-GM-130 antibody (Golgi-marker) recognised specific signal in fractions 14-17 with highest intensity in fraction 17. Calreticulin antibody (ER-marker) recognised a strong band of the expected size only in fraction 14.

Summarising, the E-110 and E-130 isoforms were differentially distributed also in the sucrose gradient fractionation. Both isoforms partially colocalised with Golgi marker (fractions 14 and 15), and the strongest signal of E-110 colocalised with ER-marker in fraction 14.

Different, the N388,406Q mutant protein strongly colocalised in Golgi-marker containing fractions (fractions 15 and 16), but almost no colocalisation with calreticulin was observed (fraction 14).

\subsubsection{Effects of the $\mathrm{N}$-linked glycosylation on intracellular distribution of hEag1 potassium channels}

\subsubsection{Intracellular localisation of the hEag1 and hEag1 mutant proteins (hEag1-N388Q, -N406Q and -N388,406Q) in CHO cells}

Glycosylation is known to be determinant for cell surface expression of many proteins, including integral membrane proteins such as ion channels (see introduction). To test whether glycosylation influences the membrane expression of the hEag1 channel, intracellular distribution was studied. Fluorescence microscopy was used to determine the subcellular localisation of the wild type and N-linked glycosylation mutants of hEag1 in CHO cells. Cells were grown for 24 hours on coverslips and transiently transfected with hEag1 and hEag1 mutant proteins (hEag1-N388Q, -N406Q and -N388,406Q). pTracer constructs were used in order to monitor transfection with GFP-Zeocin marker protein. Cells were fixed with 4\% PFA, permeabilised with $0,1 \%$ Triton $\mathrm{X}-100$ and labelled with either mAb-33 or $2413 \mathrm{x}$ 
anti-hEag1 antibody followed by appropriate fluorescently labelled secondary antibody (Alexa-Fluor 546 labelled GaM or GaR). Representative images of several independent experiments for mAb-33 and polyclonal 2413x antibody are shown in Fig. 21.

In cells transiently transfected with wild type hEag1 indirect immunofluorescence revealed widespread presence of the protein with a patched distribution of the signal throughout the cells and their processes. A similar staining pattern was observed in the N388Q mutant.

Some individual hEag1 and hEag1-N388Q transfected cells showed stronger perinuclear staining, although the spread pattern remained mostly unchanged. Our interpretation for this is that the concentrated perinuclear detection pattern in these cells was probably due to overloading the transport machinery, resulting in protein retention in some intracellular compartments.

In contrast, cells transfected with hEag1-N406Q and -N388,406Q mutants displayed an immunofluorescence pattern strongly restricted to compact perinuclear structures, presumably corresponding to the Golgi cysternae or part of the endoplasmatic reticulum (Fig. 21). In a few cells, some diffuse staining besides the perinuclear one could be detected.

These results indicate that $\mathrm{N}$-linked glycosylation at asparagine 406 is important for transport to the plasma membrane and that mutation of this site results in proteins which are retained intracellularly. Mutation of the second glycosylation site, asparagine 388, seems to have no dramatic effect on protein distribution.

As already mentioned, there were some deviations in staining pattern observed in hEag1, as well as in hEag1-N406Q and hEag1-N388Q transfected cells. Since we used polyclonal cell lines, it could be simply due to individual diversity, caused i.e. by different integration sites. On the other hand, these observations could be functionally relevant, representing some higher regulatory mechanism, where not only a particular glycosylation site, but also the relationship between the two glycosylated sites could be relevant for protein trafficking. 

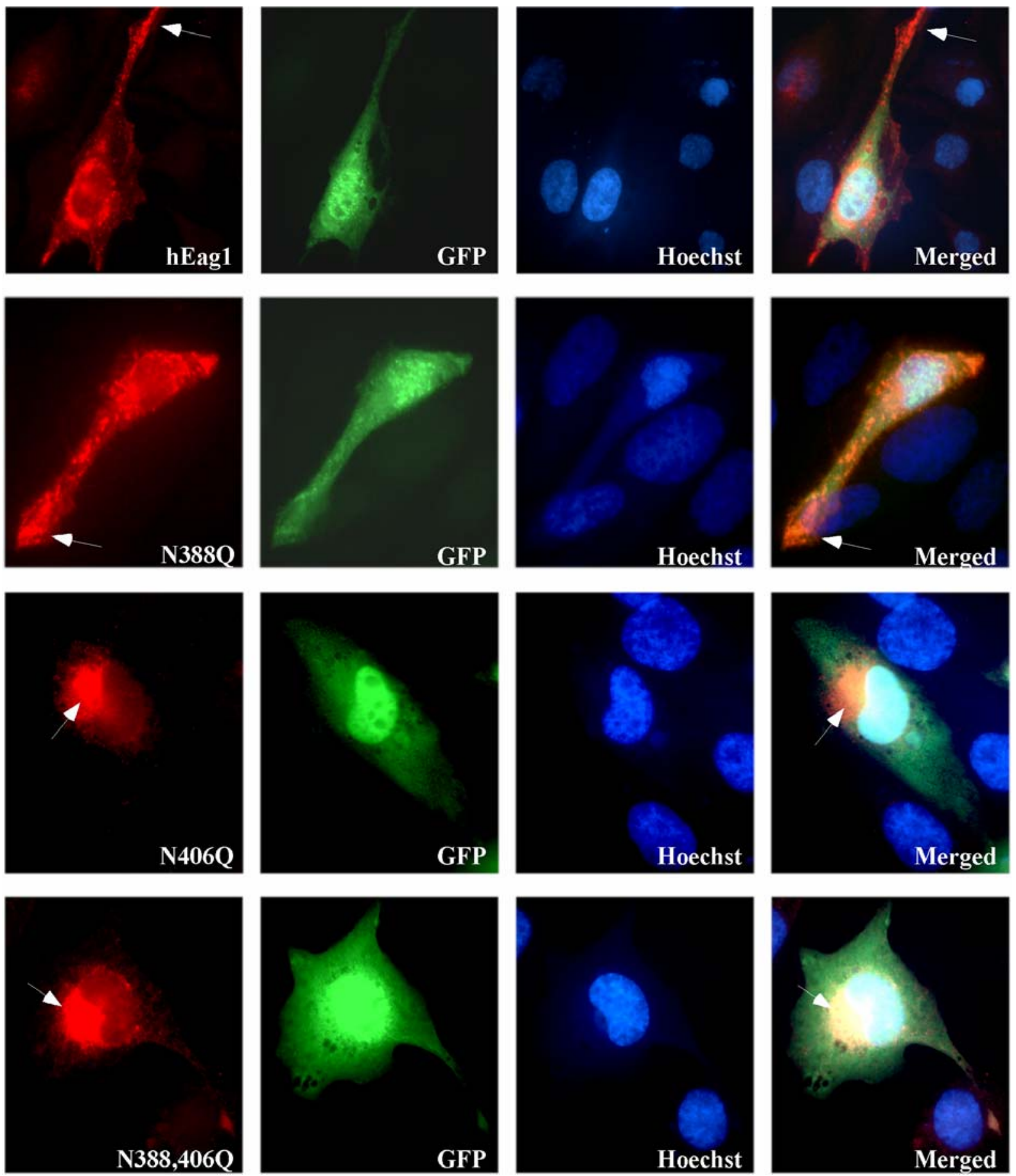

Fig. 21 Effect of N-linked glycosylation on the subcellular localisation of hEag1 and mutant proteins transiently expressed in $\mathrm{CHO}$ cells

CHO cells were grown for 24 hours on cover slips followed by transient transfection with hEag1-pTracer construct as well as with the appropriate glycosylation mutants (hEag1-N388Q, -N406Q- and -N388Q/N406QpTracer). After 24 hours cells were fixed with PFA, immunolabelled with mAb-33 antibody followed by Alexa546-conjugated secondary antibody (red). Green fluorescence corresponds to endogenous fluorescence of the GFP-Zeocin fusion protein, the marker for transfected cells, blue fluorescence corresponds to nuclei staining (Hoechst 33342).

In hEag1 and hEag1-N388Q transfected cells, arrows indicate peripheral, probably plasma membrane localisation of hEag1. In hEag1-N406Q and hEag1-N388,406Q transfected cells, arrows indicate perinuclear localisation of mutant proteins. 


\subsubsection{Characterisation of hEag1 expression in glycosylation deficient cells}

Studies of the hEag1-N406Q and -N388,406Q mutants revealed a strong effect of the complex-glycosylation on intracellular localisation of hEag1. Nevertheless, since even single amino acid exchanges in the native protein sequence could induce misfolding or aggregation and thus affect protein sorting and/or function, also unexpected effects of the introduced mutation have to be considered.

To investigate effects of $\mathrm{N}$-linked glycosylation without affecting the native protein sequence we used both internal block of N-linked glycosylation with Tunicamycin and a mutant cell line lacking parts of the glycosylation machinery.

\subsection{Internal block of the N-linked glycosylation of hEag1 in CHO cells with Tunicamycin}

Tunicamycin is a nucleoside antibiotic from Streptomyces species. It inhibits N-linked glycosylation blocking the formation of $\mathrm{N}$-glycosidic protein-carbohydrate linkages in eucaryotic cells. The optimal Tunicamycin concentration in our experiments was defined as the highest concentration allowing survival of at least $90 \%$ of cells after $24 \mathrm{~h}$, as described in 2.2.4.8.

To monitor the inhibition of N-linked glycosylation of the hEag1 channel, western blot experiments were performed on CHOhEag1 cells as well as untransfected $\mathrm{CHO}$ cells as a control. Cells were grown on Petri-dishes until they reached about $80 \%$ confluency and culture medium was then substituted by fresh medium $(5 \mathrm{ml} / \mathrm{dish}$, without antibiotic) containing either Tunicamycin $(2,5 \mu \mathrm{g} / \mathrm{ml})$ or the vehicle (DMSO) as a control. After 24 hours, cells were detached, lysed and hEag1 was immunoprecipitated from $5.000 \mu \mathrm{g}$ of cell lysates with mAb-33/62 antibody mix (5 $\mu \mathrm{g}$ each) bound to a Protein G/A-Agarose followed by western blot.

As shown in Figure 22, incubation of the CHOhEag1 cells with Tunicamycin resulted in a shift of the hEag1 migration in the SDS-PAGE gel to a position compatible with the molecular size of the non-glycosylated protein (about $108 \mathrm{kDa}$ ). The control incubation with DMSO did not affect the 2-band detection pattern of the protein. No signal was detected in untransfected $\mathrm{CHO}$ cells. 


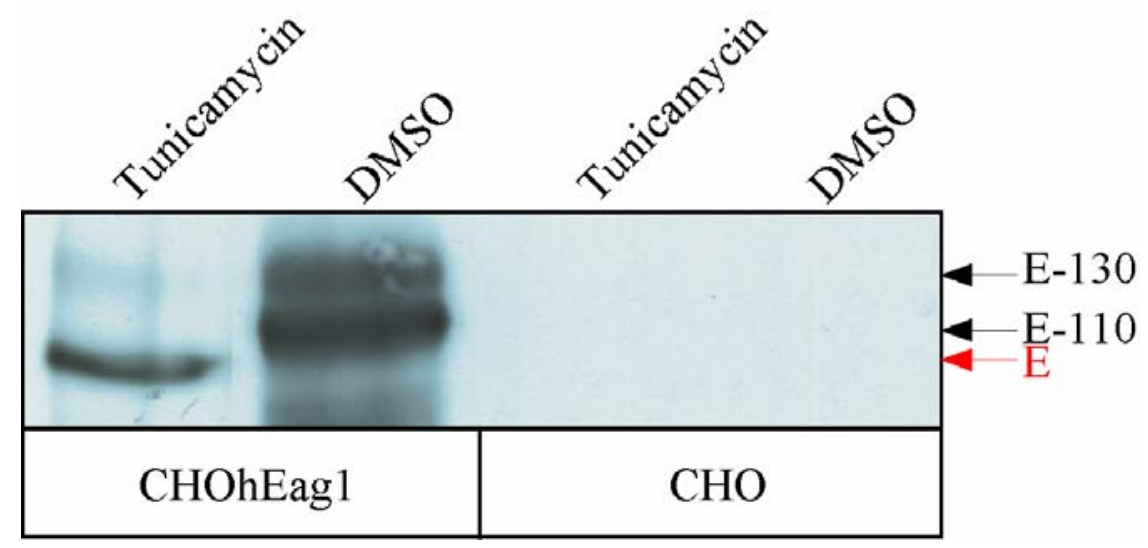

Fig. 22 Western blot of CHOhEag1 cells after internal block of $\mathbf{N}$-linked glycosylation

Cells (CHOhEag1 and wild-type CHO) were incubated either with Tunicamycin or DMSO (control) followed by the immunoprecipitation with $\mathrm{mAb}-33 / 62$ and regular western blotting.

After Tunicamycin treatment, a single band of $\approx 108 \mathrm{kDa}$, corresponding to unglycosylated $\mathrm{hEag} 1$ is detectable. In DMSO control sample, a two-band pattern typical for wild-type hEag1 (E-130 and E-110) can be seen. No signal is detectable in untransfected cells.

Additionally, subcellular localisation of hEag1 in Tunicamycin-treated cells was examined by indirect immunofluorescence with mAb-33 antibody. CHOhEag1 cells were plated on cover slips and incubated with $2,5 \mu \mathrm{g} / \mathrm{ml}$ Tunicamycin. After 17 hours the cells were fixed with PFA and immunolabelled with mAb-33 antibody either directly labelled with Cy3 (data not shown) or with mAb-33 followed by fluorescently labelled secondary antibody (Fig $23 \mathrm{~A}$, red fluorescence). Green fluorescence resulted from endogenous and independent GFP expression (a component of pTracer vector).

As can be seen in Figure 23, staining with mAb-33 antibody revealed a strong rearrangement of hEag1 expression after Tunicamycin treatment when compared to DMSO treated cells (Fig. 23 B). In Tunicamycin treated cells, hEag1 was detectable only in compact perinuclear structures, showing staining pattern similar to the one observed in N406Q and N388,406Q mutants. The perinuclear staining was even more pronounced in Tunicamycin treated cells than in the glycosylation mutant.

These results suggest that the strong trafficking impairment observed in Tunicamycin treated $\mathrm{CHO}$ cells can contribute to the Tunicamycin-mediated block of N-linked glycosylation. The more obvious perinuclear localisation of hEag1 in Tunicamycin treated cells could be explained by additional effects of this drug on cellular metabolism. 


\section{Tunicamycin}

A
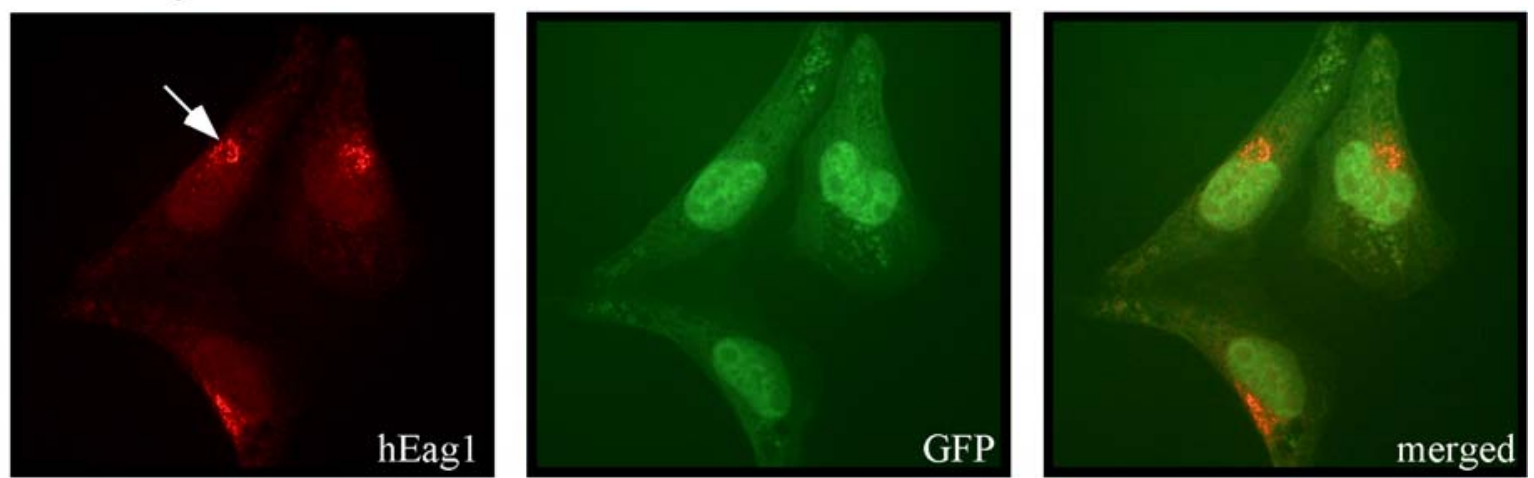

\section{DMSO control}
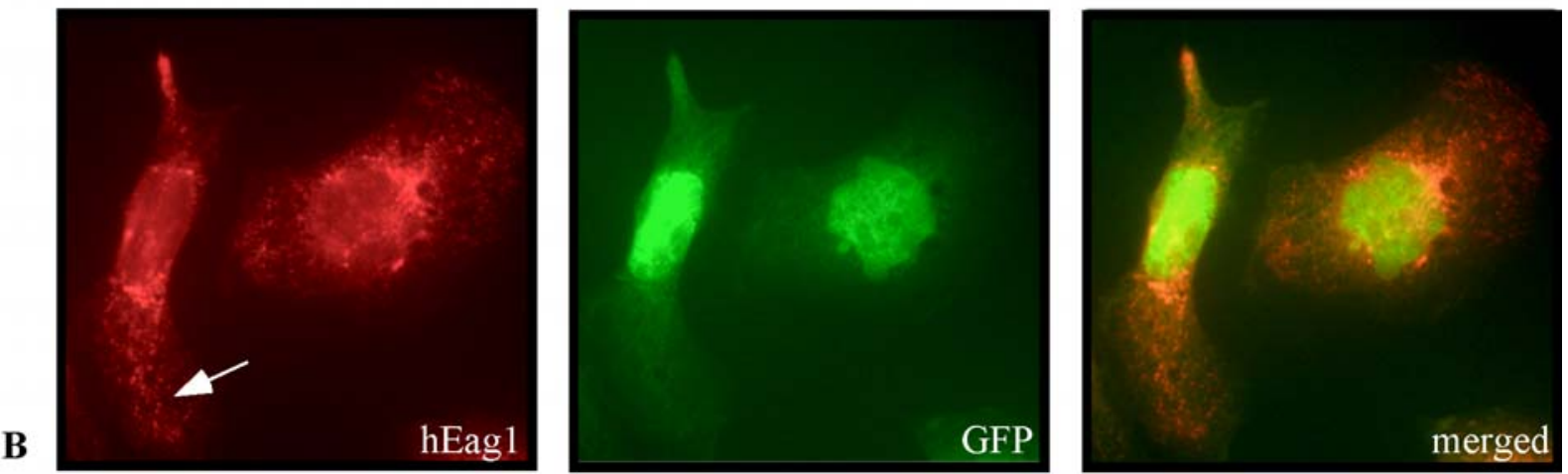

Fig. 23 Immunofluorescent localisation of the hEag1 protein in Tunicamycin treated CHO cells.

CHO-hEag1 cells were grown for $17 \mathrm{~h}$ in culture medium enriched either with Tunicamycin $(2,5 \mu \mathrm{g} / \mathrm{ml})$ or with DMSO (control). Immunolocalisation was performed on PFA fixed cells, using the mAb-33 antibody detected with fluorescently labelled secondary antibody (Alexa Fluor-546, red). Green fluorescence corresponds to the GFP selection marker expression showing both cytoplasmatic and nuclear localisation. All images were taken using a fluorescence microscope.

A) Tunicamycin treated CHOhEag1 cells

B) Control DMSO treatment

Anti-hEag1 antibody detected compact structures clearly localising to the perinuclear compartment (compare with GFP) only in cells incubated with Tunicamycin. Control cells show a diffused distribution pattern typical for Eag1.

\subsection{Expression of hEag1 in glycosylation-deficient Lec1 cells}

Lec1 cells are a mutant clonal derivative from the $\mathrm{CHO}$ clone Pro-5 (ATCC, Rockville, USA). Lec1 cells lack the GlcNAc-T1 glycosyltransferase, so that N-linked carbohydrates are blocked at the dolichol carrier, and N-linked glycosylation of the proteins does not occur.

Lec1 cells were stably transfected with the hEag1-pTracer construct (Lec1hEag1 cell line) and expression of hEagl was tested by western blot. 
Since Lec1 cells lack the native glycosylation machinery, effects of the mutation of hEag1 glycosylation sites were expected to be mimicked by the expression of hEag1 in Lec1 cells. Indeed, after immunoprecipitation with mAb-33/62 antibody mix (5 $\mu \mathrm{g}$ each) from $5000 \mu \mathrm{g}$ of whole-cell lysates and separation by a 7,5\% SDS-PAGE gel, a single band of approximately $108 \mathrm{kDa}$ corresponding to the unglycosylated hEag1 protein (Fig. 24) was detectable on western blot. No signal was detected in untransfected Lec1 cells.

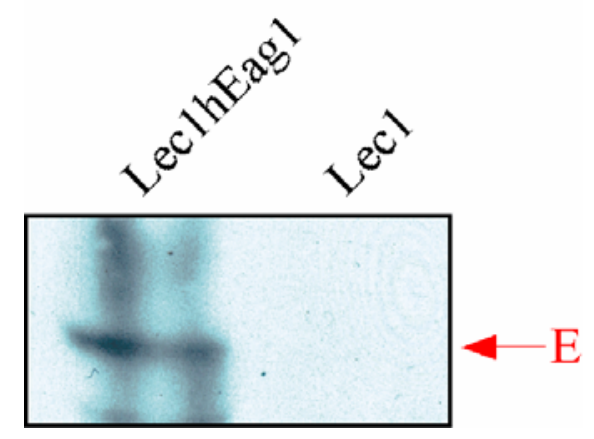

Fig. 24 Western blot analysis of the hEag1 protein immunoprecipitated from glycosylation deficient Lec1hEag1 cells.

Regular western-blot of Lec1hEag1 and wild-type Lec1 cells immunoprecipitated with mAb-33/62 followed by the immunodetection with $2413 x$ polyclonal antibody. A single band of $\approx 108 \mathrm{kDa}$ (E, red arrow), corresponding to the unglycosylated hEag1 protein was detected only in Lec1hEag1 and not in untransfected cells.

To test the intracellular localisation of hEag1, permanently transfected Lec1hEag1 were plated on covereslips and allowed to attach for $24 \mathrm{~h}$, followed by the immunofluorescent localisation with mAb-33 antibody directly labelled with Cy3 (red). In these experiments, hEag1 was also found to localise in compact perinuclear structures (Fig. $25 \mathrm{~A}$ ), whereas the wide-spread distribution pattern of hEag1 detectable in CHO cells was completely lost, as in the Tunicamycine-treated CHOhEag1 or in glycosylation deficient mutants expressed in $\mathrm{CHO}$ cells. The untransfected Lec1 cells showed no staining with the hEag1 antibody (Fig. 25 B).

Taken together, these results strongly suggest that the effects observed in hEag1 mutants are indeed due to the disruption of the glycosylation sites and not to some allosteric effects of the introduced mutation. 

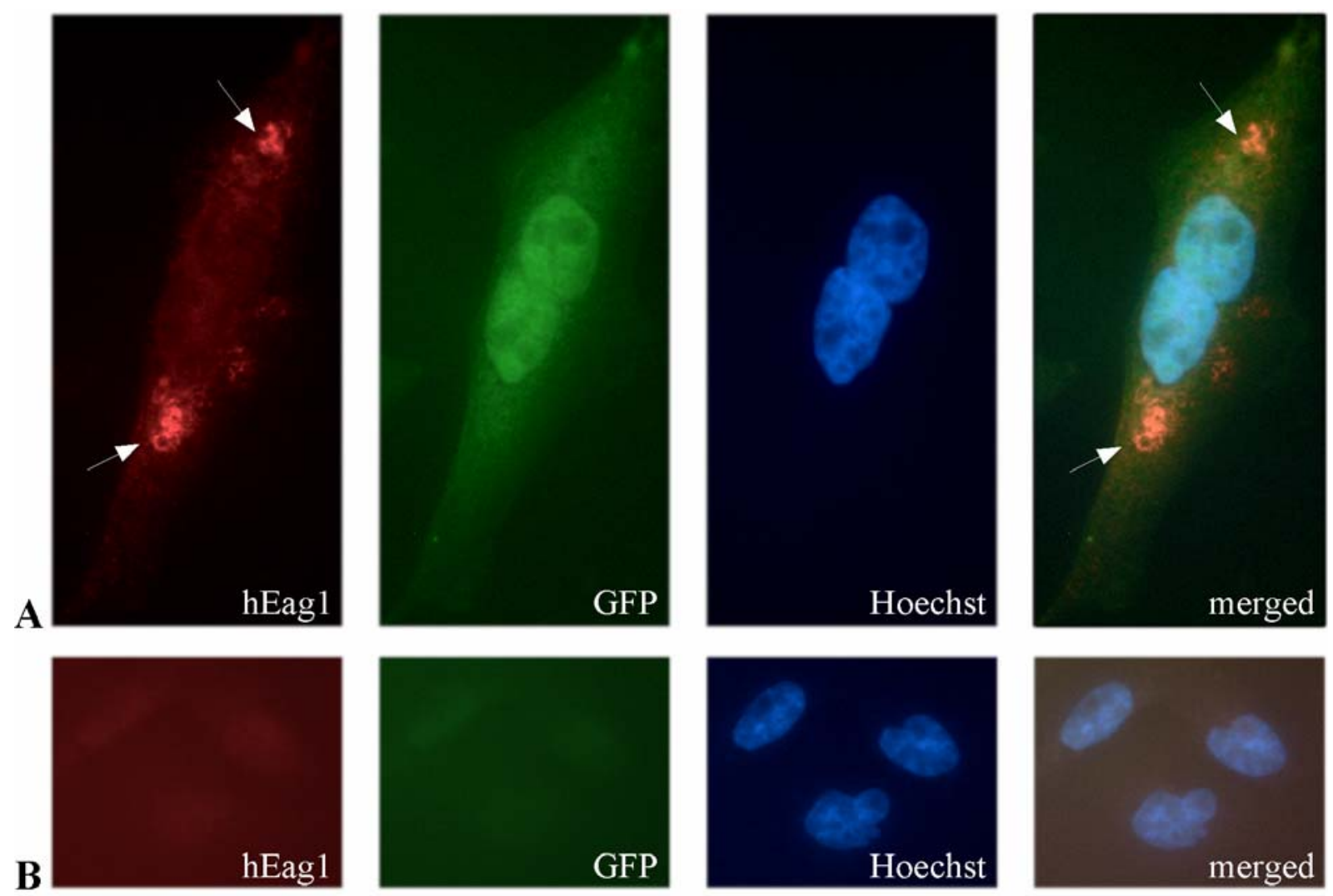

Fig. 25 Immuofluorescent localisation of hEag1 in permanently transfected Lec1 cells

Indirect immunofluorescence of hEag1 was performed on PFA fixed Lec1 cells using mAb-33-Cy3 antibody. Immunolocalisation is merged with internal GFP fluorescence of marker fusion-protein and nuclear staining (Hoechst). In the Lec1hEag1 cells (upper panel), mAb-33 anti-hEag1 antibody recognised compact perinuclear structures (indicated by arrows). Untransfected Lec1 cells did not stain with mAb-33-Cy3 antibody (upper panel).

A) Lec1-hEag1 cells

B) Lec1 wild-type cells

\subsection{Colocalisation of hEag1 and mutant proteins with different intracellular markers}

To study whether transport-deficient hEag1 glycosylation mutants are either trapped in defined transport organelles or are directed to proteolytic degradation, or undergo some other management, we analysed the subcellular localisation of glycosylation mutants with respect to different organelles. We used single glycosylation-mutants, N388Q and N406Q, in order to monitor the contribution of a particular glycosylation site to the intracellular distribution in detail. 
pTracer vector was inconvenient for double immunostaining experiments because it encodes a independent GFP-Zeozin fusion protein. Thus hEag1 as well as generated hEag1glycosylation mutants were subcloned into the pCDNA4 myc/HIS B+ vector, which generates a fusion protein with c-myc tag at the C-terminal end. For this the hEag1 stop codon had to be replaced by an appropriate restriction site (XhoI). To ensure a continuous ORF an appropriate insert was generated by PCR using CMV forward and the XhoSTOP primer. This insert was then subcloned into the pCDNA4 vector by KpnI-XhoI restriction sites.

To ensure proper protein expression and processing, hEag1-pCDNA4 as well as N388QpCDNA4 and N406Q-pCDNA4 clones were stably transfected into CHO cells and analysed by immunoprecipitation followed by regular PNGase F treatment and western-blotting. The 2413x polyclonal antibody detected a similar band pattern as in the previous experiments (data not shown), confirming correct expression of hEag1 glycosylation isoforms. The c-myc antibody failed to detect the hEag1 protein on western-blots. This can be caused by some posttranslational trimming of the fusion protein.

Immunofluorescence studies were performed on PFA (Fig. 26 A-F) or methanol (Fig. 26 G and $\mathrm{H}$ ) fixed $\mathrm{CHO}$ cells transiently expressing hEag1 and glycosylation mutants N388Q and N406Q. Wild-type hEag1 as well as mutant proteins were detected with mAb-33 followed by Cy3 secondary antibody (red) or 2413x polyclonal antibody (for colocalisation with GM-130, performed with monoclonal antibody) followed by Alexa Fluor 488 (green) labelled secondary antibody. Additionally, major trafficking organelles: endoplasmatic reticulum (TRAP $\alpha$, calreticulin, p53), Golgi (GM-130), early endosomes (Rab5), late endosomes (Rab7), and lysosomes (Cathepsin D and Limp2) were labelled with specific antibodies followed by Alexa Fluor 488 (green) or by Alexa Fluor 546 (for GM-130 antibody) secondary antibodies. Additionally, in almost all experiments besides colocalisation with GM130 and TRAP $\alpha$, nuclei were stained with Hoecht 33342 (not shown). This is probably also visible in the green channel, since a green nuclear staining is visible in all mentioned cases.

As shown in Fig. 26, the detection pattern of wild type and mutant proteins was similar to one in previous experiments. hEag1 and the N388Q mutant gave a widespread spotted distribution pattern, whereas localisation of the N406Q mutant was reduced to perinuclear structures. 
All three hEag1 forms partly co-localised with endoplasmatic reticulum markers (Fig. 26 A-C), what was expected since plasma-membrane proteins are synthesised by ER-resident ribosomes. However, co-localisation of the N406Q mutant protein with the endoplasmatic reticulum was more prominent, with almost no staining outside the ER. This result suggests that the core-glycosylated N406Q mutant protein can be trapped in the endoplasmatic reticulum and thus fail to reach the membrane. All three tested proteins only slightly co-localised with the Golgi marker, GM-130. Rab5 and Rab7 proteins, supposed to stain early and late endosomes, respectively, showed besides the expected signals also a strong nuclear staining, (as mentioned, probably due to Hoechst 33342 staining visible in the greenfluorescence channel). All hEag1 isoforms showed some co-localisation with the Rab7 antibody, mostly in perinuclear regions, but because of the nuclear staining of Rab7, no conclusion on the specificity of the co-localisation can be drawn.

Similarly, hEag1 and both glycosylation mutants N388Q and N406Q showed only a slight co-localisation with the lysosomal markers, Cathepsin D and Limp 2.
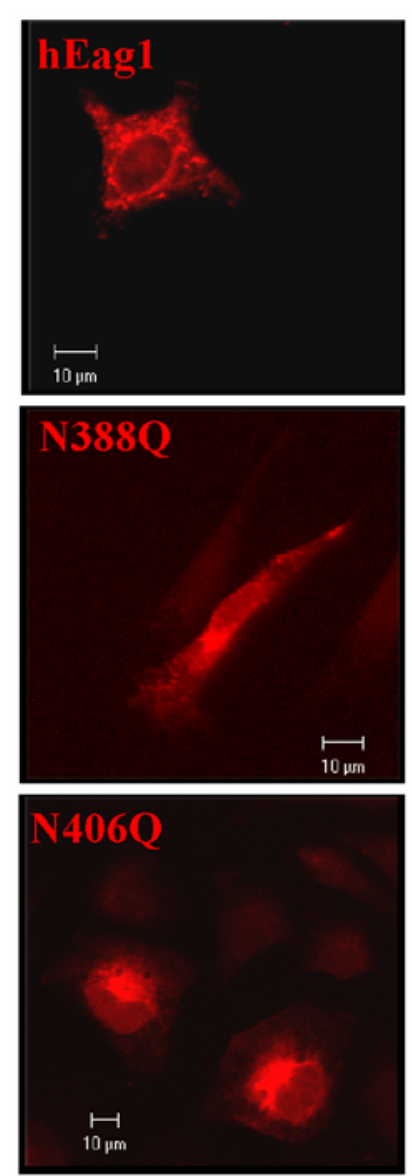
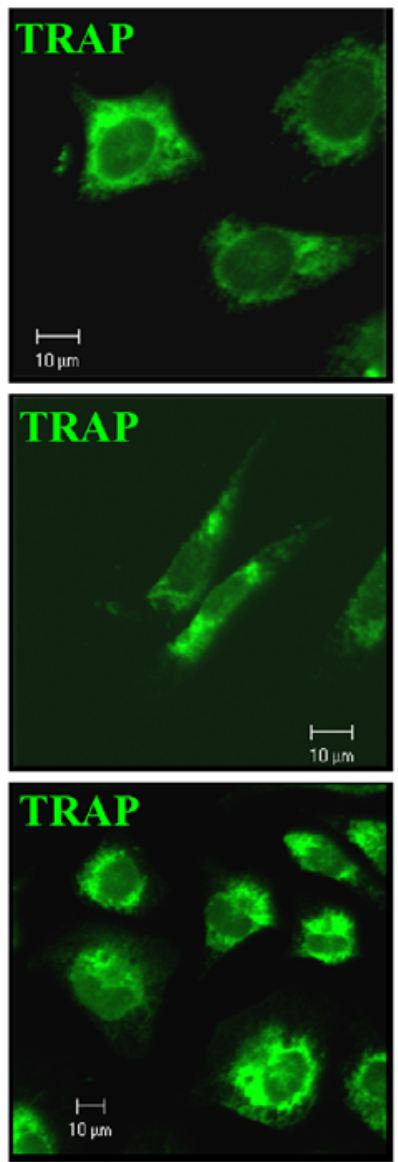
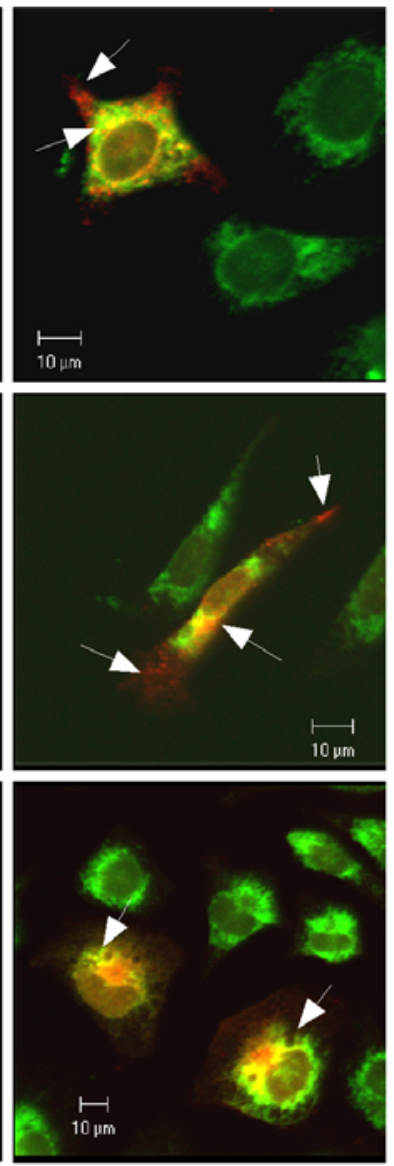

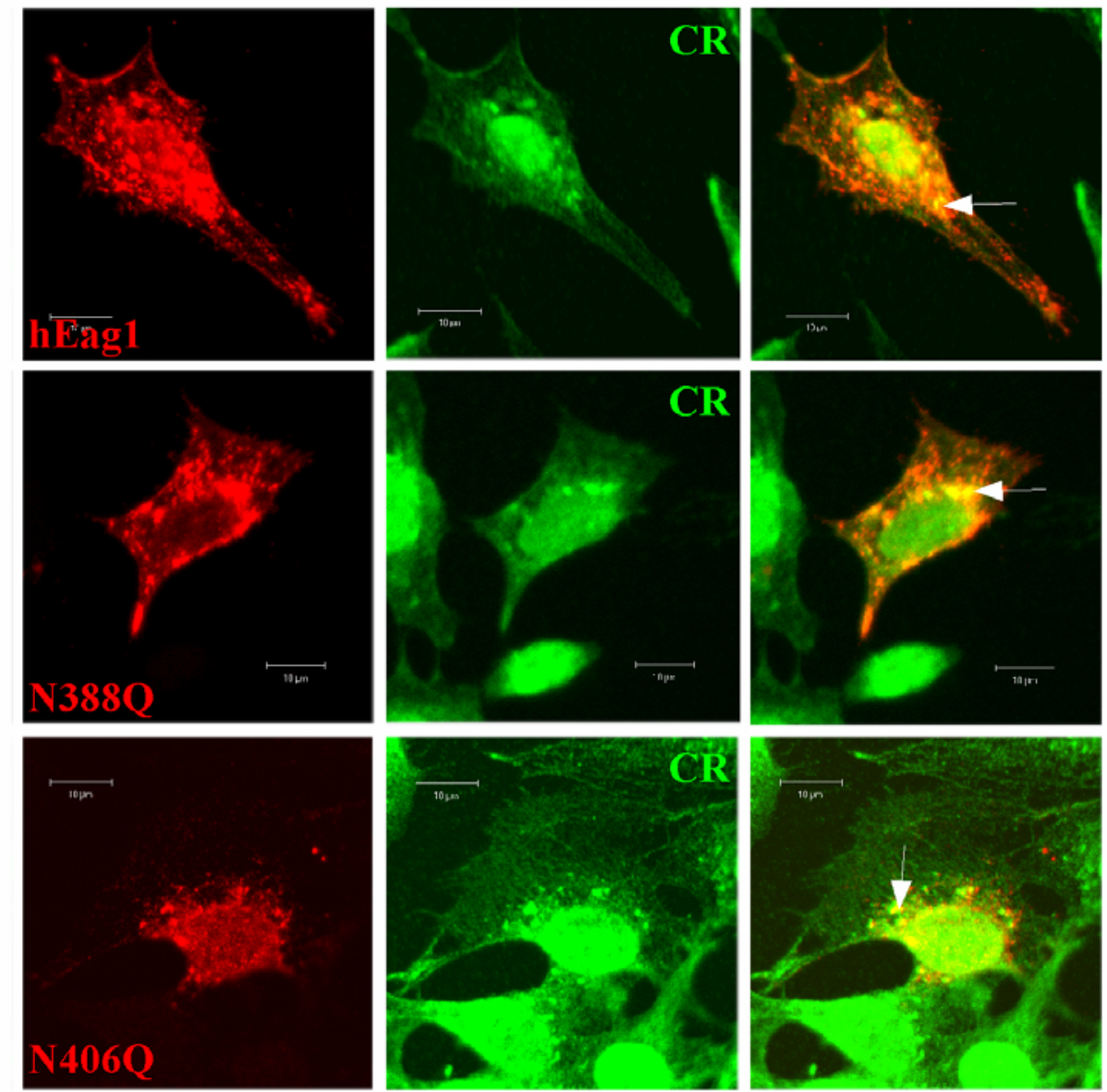

B
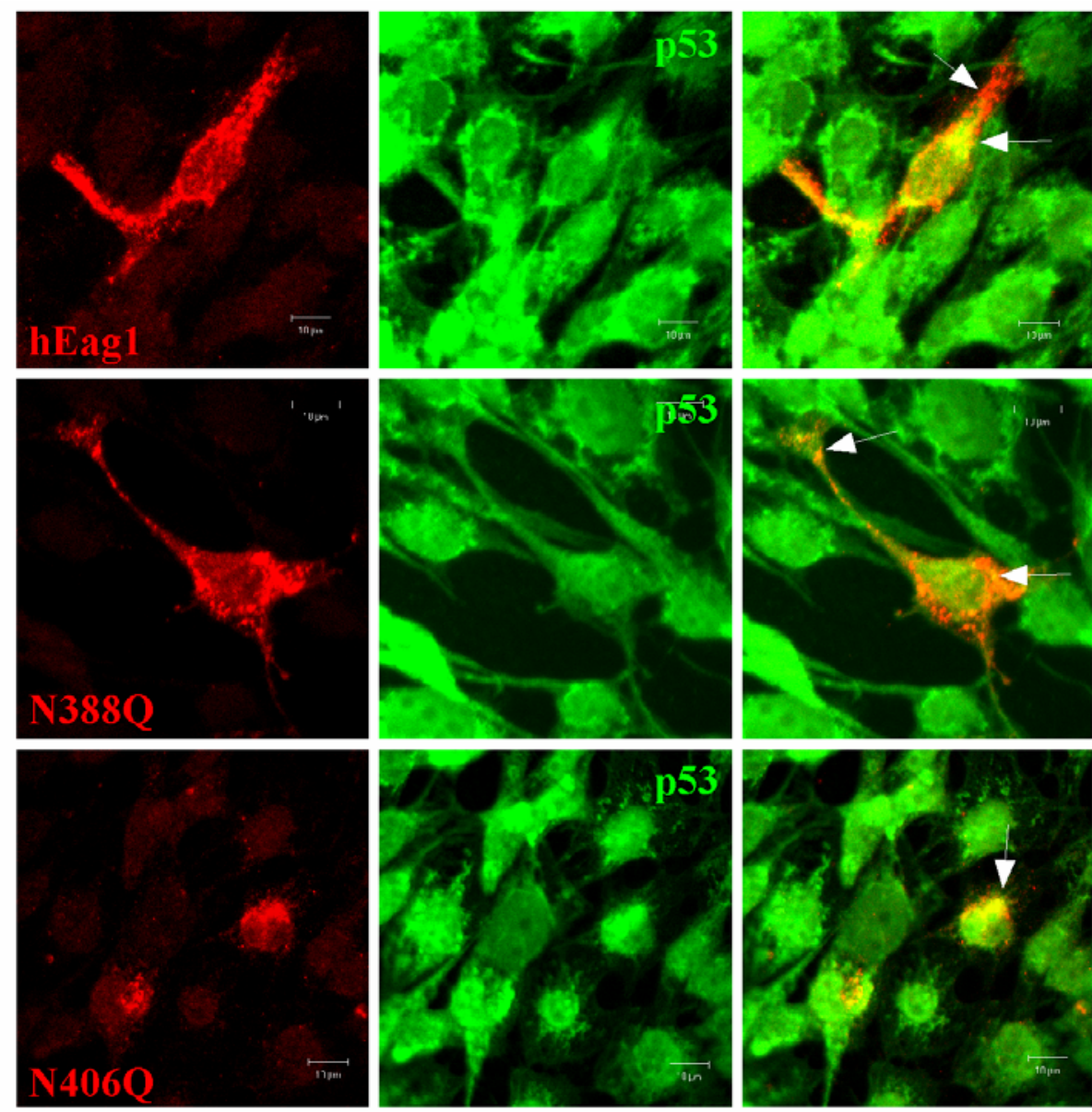

C 

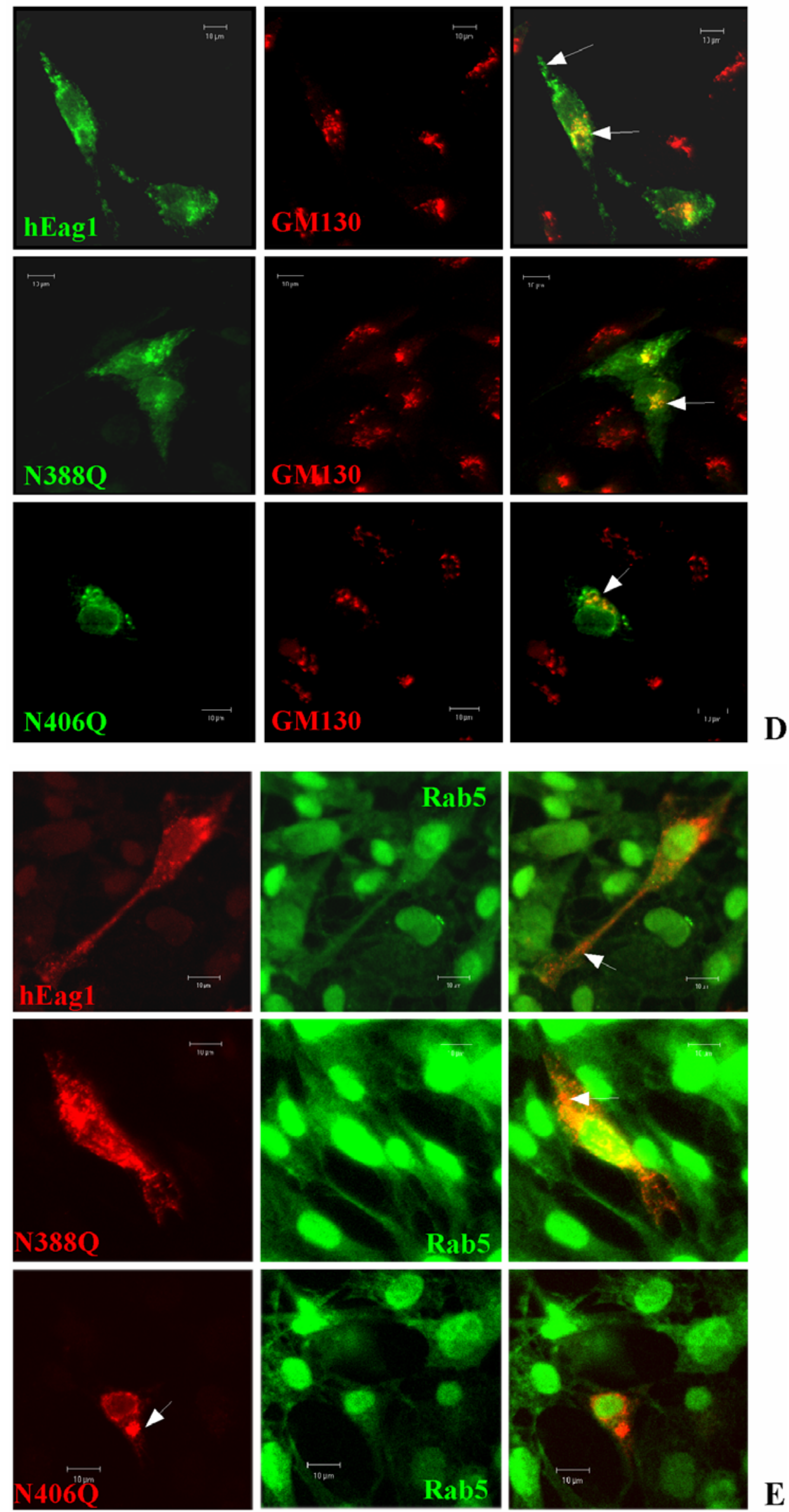

E 

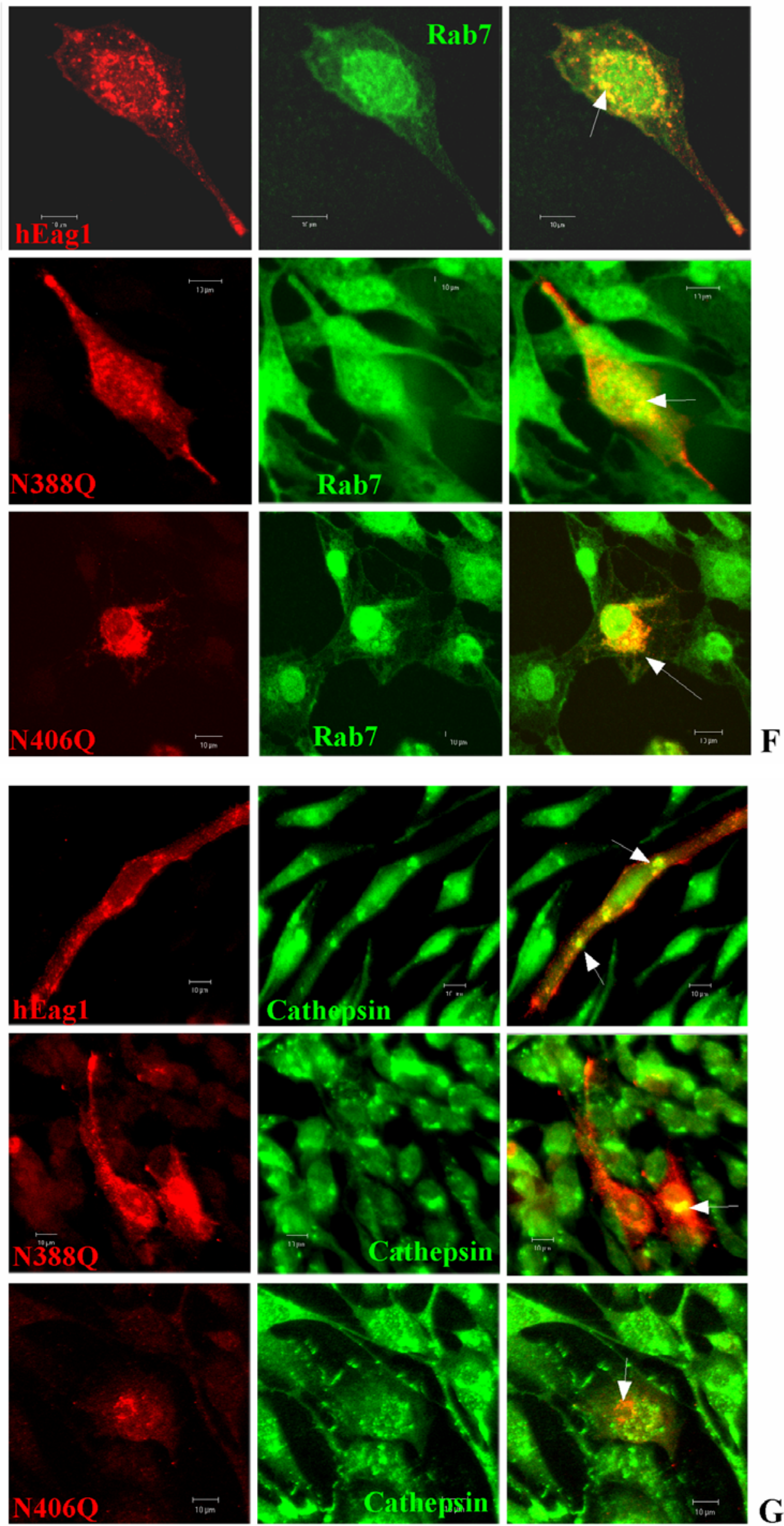

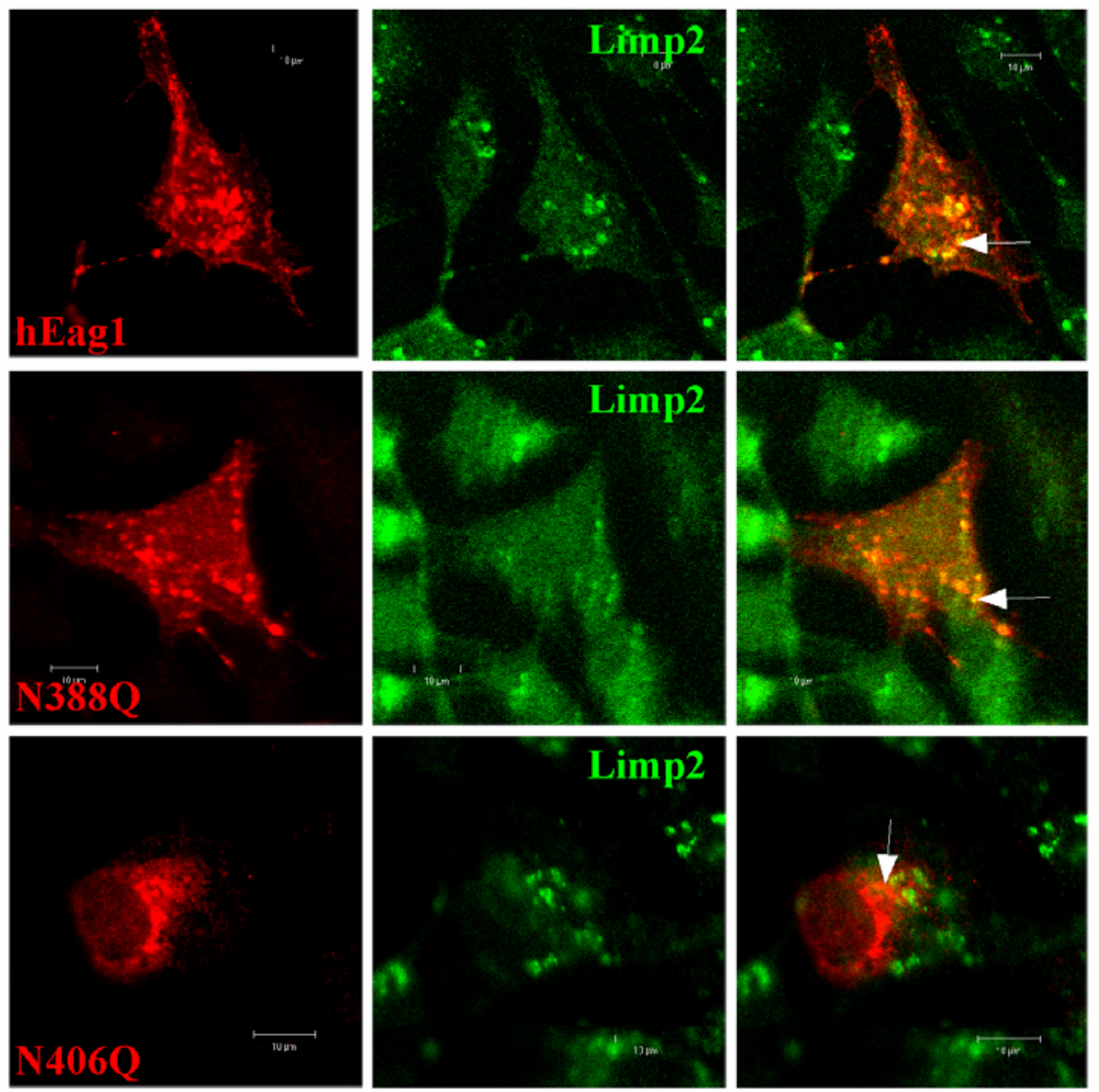

$\mathbf{H}$

Fig. 26 Colocalisation of hEag1 and mutant proteins (N388Q and N406Q) with intracellular markers CHO cells were transiently transfected with hEag1-, N388Q- and N406Q-pCDNA4. 48 hours after transfection cells were fixed with PFA (A-F) or $\mathrm{MeOH}(\mathrm{G}$ and $\mathrm{H})$, followed by staining with an Eag1 specific antibody (mAb-33 or 2413x). To monitor subcellular localisation cells were labelled in parallel with antibodies against:
A) TRAP $\alpha$ (endoplasmatic reticulum)
B) Calreticulin (CR; endoplasmatic reticulum)
C) p53 (protein $53 \mathrm{kDa}$; endoplasmatic reticulum)
D) GM-130 (Golgi)
E) Rab5 (early endosomes)
F) Rab7 (late endosomes)
G) Cathepsin D (lysosomes)
H) Limp2 (lysosomes)

Taken together, the above results suggest that the complex glycosylated N388Q-mutant behaves similar to the wild-type hEag1 protein showing partial colocalisation with the endoplasmatic reticulum as well as slight co-localisation with endosomal and lysosomal markers.

In contrast, the core-glycosylated N406Q mutant seems to be trapped in the endoplasmatic reticulum, probably due to the abolished complex glycosylation caused by introduced mutation. 


\subsubsection{Functional characterisation of hEag1 and glycosylation deficient hEag1 potassium channels}

To study effects of N-linked glycosylation on the functional properties of hEag1 potassium channel, both Xenopus laevis oocytes and mammalian cells were chosen as a heterologous expression system. The characterisation of the currents from putative glycosylated and unglycosylated hEag1 channels was measured using standard electrophysiological techniques.

\subsubsection{Heterologous expression of the hEag1 and glycosylation mutants in Xenopus laevis oocytes}

Full-length cDNA of hEag1 and the glycosylation mutants hEag1-N388Q and hEag1-N406Q were subcloned into the PSGEM expression vector (single step cloning of BamHI-SmaI insert from appropriate pTracer clone) and cRNA was prepared by in vitro transcription (see 2.2.2.7). Integrity of the synthesised cRNA was tested on agarose gels, and cRNA was finally quantified using Ribo Green ${ }^{\mathrm{TM}}$ assay. Oocytes were surgically extracted and, after removal of the follicular layer, microinjected with $50 \mathrm{nl}$ of the synthesised cRNA. Macroscopic currents mediated by hEag1 and mutant channels were measured 3 days after cRNA injection by using the Two-Electrodes Voltage Clamp (TEVC) technique.

Fig. 27 A shows representative current traces recorded from oocytes injected with $50 \mathrm{nl}$ of hEag1, hEag1-N388Q and hEag1-N406Q cRNA (350 ng/ $\mu$; see 2.2.6.1). Currents were obtained in response to $500 \mathrm{~ms}$ depolarising pulse to $+60 \mathrm{mV}$ from a holding potential of $-80 \mathrm{mV}$. All the macroscopic currents obtained from oocytes expressing either hEag1 or mutant channels exhibited characteristics similar to those previously described in the literature for the hEag1 channels, meaning: voltage-dependence of outward currents with rectification, ColeMoore-like effect and absence of inactivation. However, peak current amplitudes were significantly smaller when hEag1-N406Q channels were analysed and compared to hEag1 currents (Fig. 27 A). 

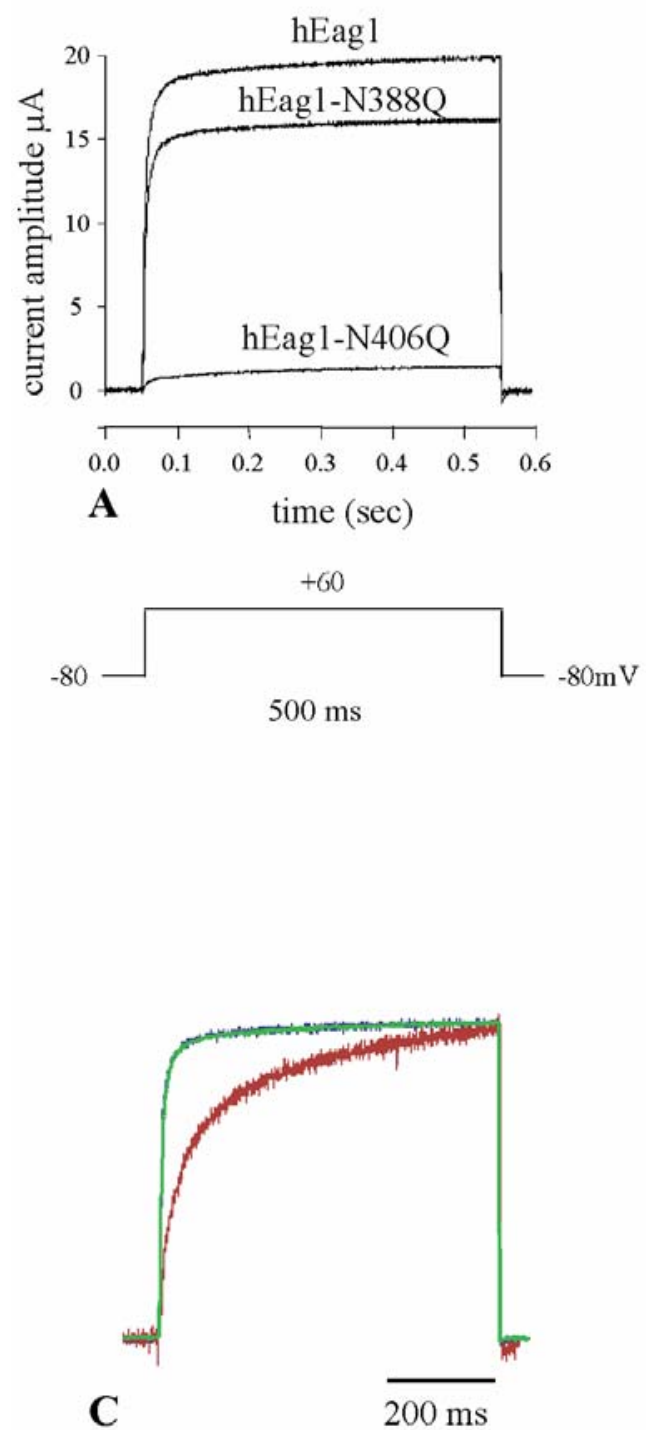

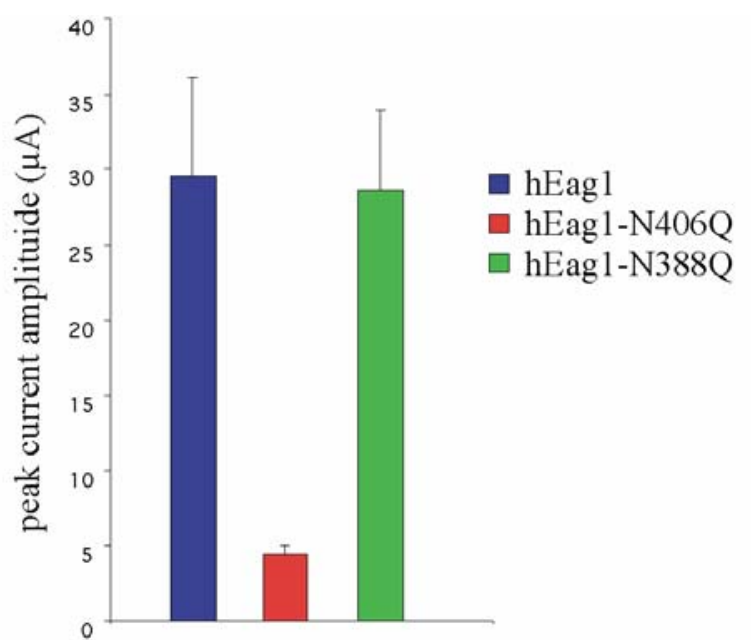

B

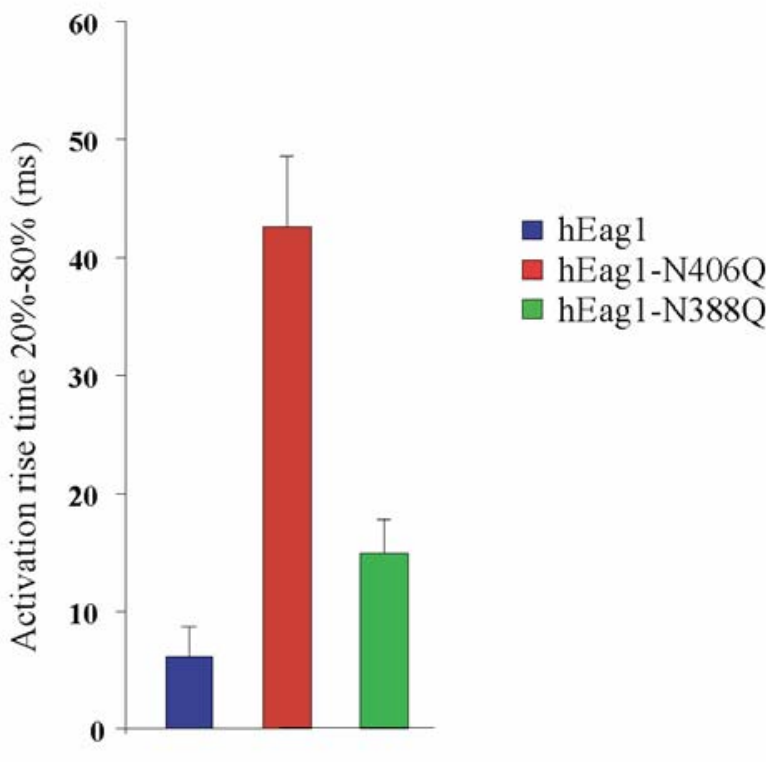

D

Fig. 27 Two-electrodes voltage clamp recordings from wild-type and mutant hEag1 channels expressed in Xenopus laevis oocytes

Currents were recorded by two-electrode voltage clamping in response to single $500 \mathrm{~ms}$ depolarising pulses from -80 to $+60 \mathrm{mV}$ (inset).

A) Representative current traces mediated by hEag1 wild-type channels and glycosylation hEag1 mutants.

B) Statistical evaluation of the maximum peak current amplitudes obtained from 12 recordings. Mean for hEag1 is $29.6 \mu \mathrm{A}$ for hEag1-N406Q is $4.5 \mu \mathrm{A}$ and for hEag1-N388Q mutant, $28.6 \mu \mathrm{A}$. The standard error is represented by bars.

C) Slowing down of the activation of hEag1-N406Q mutant channels. Current traces (A) were normalised to the maximal peak current amplitudes and superimposed for comparison of the activation kinetics.

D) Statistical evaluation of $20-80 \%$ values for the analysed oocytes

Statistical evaluation of a series of measurements in different oocytes (12 for each: hEag1, hEag1-N388Q and hEag1-N406Q) are presented on the Fig 27 B. The average peak current amplitudes obtained from hEag1-N406Q channels $(4.47 \mu \mathrm{A})$ represented about $15 \%$ of the 
maximal peak current mediated by the hEag1 channels $(29.56 \mu \mathrm{A})$. In contrast recordings of the hEag1-N388Q mutant $(28.63 \mu \mathrm{A})$ resembled those obtained from the wild-type channels. Additionally closer inspection of the normalised and superimposed representative current traces, shown on the Fig. $27 \mathrm{C}$, reveals that the time course of the hEag1-N406Q mutant current is strongly modified, exhibiting a significant slowing down of the channel activation. The changes in the temporal course of the activation process were estimated by measuring the time interval, elapsed between $20 \%$ and $80 \%$ of maximal current amplitude (activation rise time 20-80\%). As can be seen in Fig. 27 D, the activation of the hEag1-N406Q mutant channels was significantly slowed down; the channels needed about 6 fold longer time to reach $80 \%$ of maximal current amplitude. For the hEag1-N388Q mutant channels only a slight slow-down in the activation was observed.

\subsubsection{Voltage dependence of hEag1-N406Q mutant channels}

To determine the voltage dependence of activation, $500 \mathrm{~ms}$ depolarising voltage-pulses were applied from a holding potential of $-80 \mathrm{mV}$ to -70 to $+80 \mathrm{mV}$, in $10 \mathrm{mV}$ increments, in presence of $30 \mathrm{mM}$ external potassium and with $20 \mathrm{sec}$ intervals.

At the end of each pulse, peaks of inward (tail) currents were determined at a constant hyperpolarising membrane potential of $-80 \mathrm{mV}$. To analyse the voltage dependence of the activation, values of the recorded inward peak currents were normalised to the maximum amplitude and plotted against the membrane potential of the corresponding $500 \mathrm{~ms}$ depolarising pulses. Normalised peak current amplitudes were averaged for several episodes $(n=5-8)$.

As shown in Fig. 28, mutation of the N406 glycosylation site induced not only a slowing down of the activation kinetics of hEag1 but also influenced the voltage dependence of activation. The voltage necessary to reach half of the maximum conductance $\left(V_{50}\right)$ was shifted about $25 \mathrm{mV}$ towards more depolarising potential when comparing hEag1-N406Q with wild type channels. In contrast, no dramatic changes were observed in the voltage dependence of hEag1-N388Q mutant channels. Only a slight shift $(5 \mathrm{mV})$ of the equilibrium potential towards more depolarising potential was observed, although the statistical significance of the shift is not clear. 


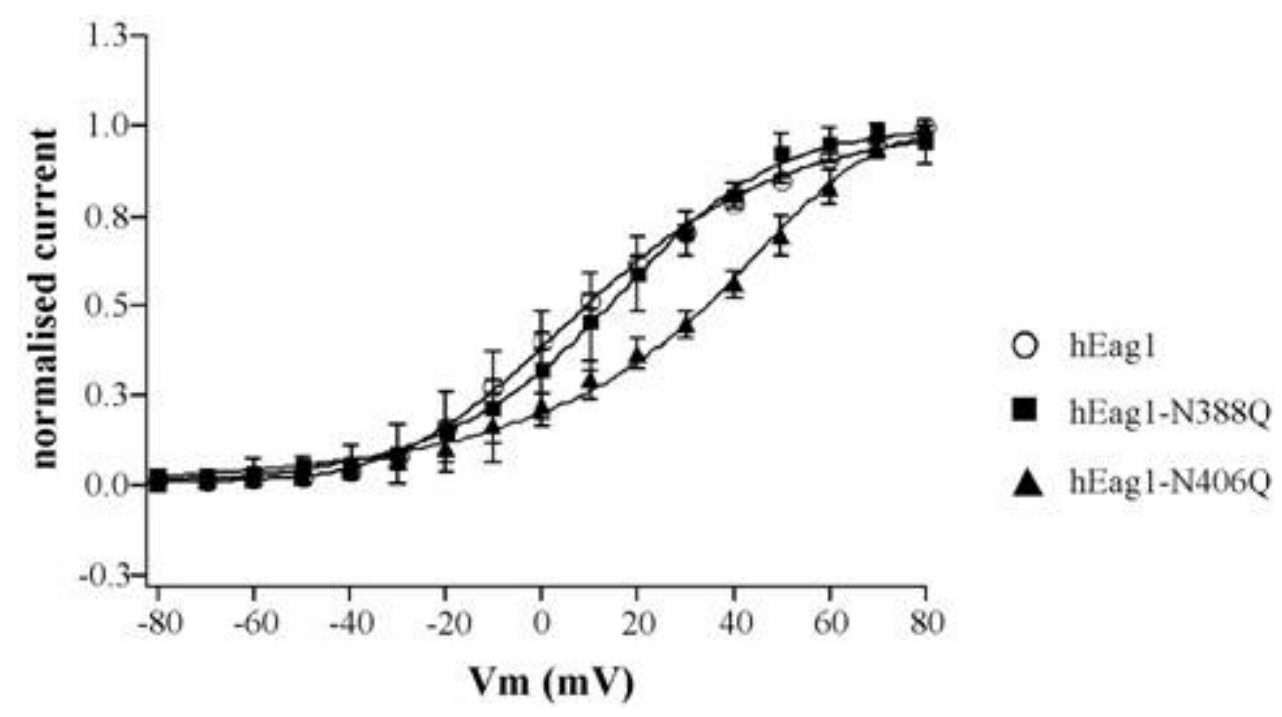

Fig. 28 Voltage dependence of hEag1 and hEag1-N406Q channels activation

From a holding potential of $-80 \mathrm{mV}$, macroscopic currents were elicited by applying depolarising pulses of increasing amplitudes, to the indicated membrane potentials $(\mathrm{Vm})$, followed by a constant hyperpolarising pulse to $-80 \mathrm{mV}$. Values for each inward peak current were normalised to the maximal tail current amplitude and plotted against the corresponding membrane potential. Note the shift of the hEag1-N406Q activation curve (25 mV towards more depolarising potentials) when compared to the wild type channels.

\subsubsection{Functional characterisation of the glycosylation deficient hEag1 in mammalian cells}

Besides the analysis in the Xenopus laevis oocytes heterologous expression system, effects of the N-linked glycosylation of the hEag1 on the electrophysiological properties of the channel were also studied in mammalian cells. Following glycosylation-deficient cells were examined:

- CHOhEag1 after Tunicamycine treatment $(2,5 \mu \mathrm{g} / \mathrm{ml}$ for at least $15 \mathrm{~h})$,

- CHOhEag1-N388,406Q (glycosylation mutant),

- Lec1hEag1 (glycosylation deficient),

- CHOhEag1 cells as a control.

Cells were grown on Ø $1 \mathrm{~cm}$ cover-slips at low density to enable single cell measurements. The whole-cell configuration of the patch-clamp technique was used, in which channel openings can be induced by controlling the membrane potential and the sum of the ionic currents corresponding to all channels present on the cell membrane can be recorded. In all the experiments, the membrane was kept at a holding potential of $-80 \mathrm{mV}$, where the probability of the hEag1 channels to be in a resting state is very high. From a holding potential of $-80 \mathrm{mV}$, different voltage protocols, which are schematically depicted in Fig. 29 A (inset), 
were applied to elicit and record the ionic currents flowing through activated channels. The duration of these pulses varied between 500 and 2000 ms (see time scale in corresponding graphs). Representative current traces are shown in Fig. 29 A.

Under these experimental conditions, current densities (pA/pF) were determined for each cell. Values were calculated from peak current amplitudes obtained at the steady-state of activation in response to a depolarising pulse to $+80 \mathrm{mV}$ and divided by the value of cell membrane capacitance, which was estimated after automatic $C_{\text {slow }}$ compensation. As can be seen in the Fig. 29 B, a dramatic reduction in current density was observed in all cases where glycosylation of hEag1 was abolished (either by double mutation, incubation with Tunicamycin or expression in Lec1 cells).

Additionally, the dependence of the activation kinetics on membrane potential (Cole-Moorelike effect) was examined. For this, a depolarising pulse of 1 sec in duration and $+80 \mathrm{mV}$ in amplitude was delivered after 2 sec hyperpolarisation either to -140 or $-60 \mathrm{mV}$. As can be observed in Fig 29, in all the unglycosylated conditions, channels presented a marked slowing down of the activation kinetics. Moreover in the hEag1-N388,406Q mutant channels, as well as in the hEag1 channels expressed in Lec1 cells, a less pronounced Cole-Moore-like effect is seen.

These results are in agreement with those obtained from Xenopus oocytes and indicate that $\mathrm{N}$-glycosylation is not only involved in the mechanism of hEag1 channels transport to the cell membrane but also participate in the fine modulation of some pivotal biophysical channel properties, such as activation kinetics and voltage dependence. Besides, since the N-linked glycosylation is associated with attachment of a large and often charged sugar structure, which in hEag1 channels is positioned near by the channel pore, the structural presence of oligosaccharide could modify functional properties of the channel.

Moreover, the structural modifications induced by the amino acid substitution itself (in the glycosylation deficient mutants), cannot be a source of the observed changes, since similar functional effects were observed when wild-type channels were expressed in glycosylation deficient cells. 


\section{CHOhEag1}

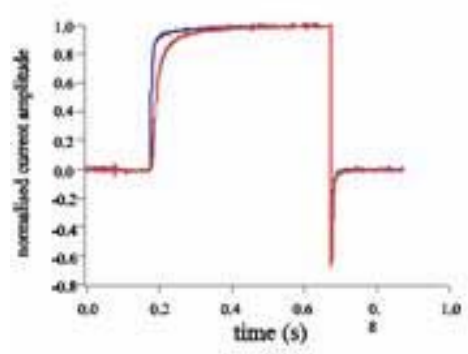

Lec1hEag1

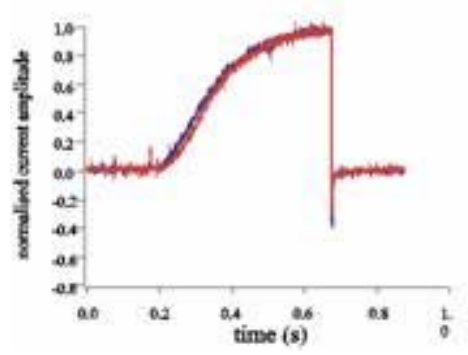

\section{CHOhEag1-N388,406Q}

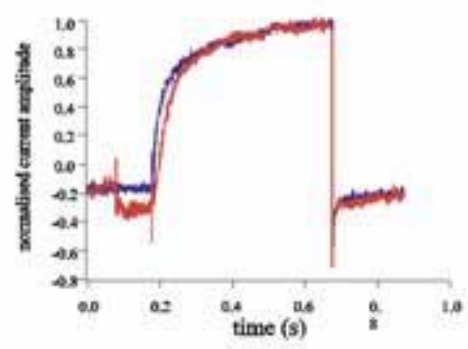

CHOhEag1 Tunicamycin

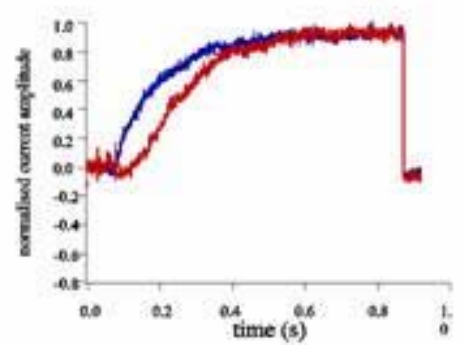

A

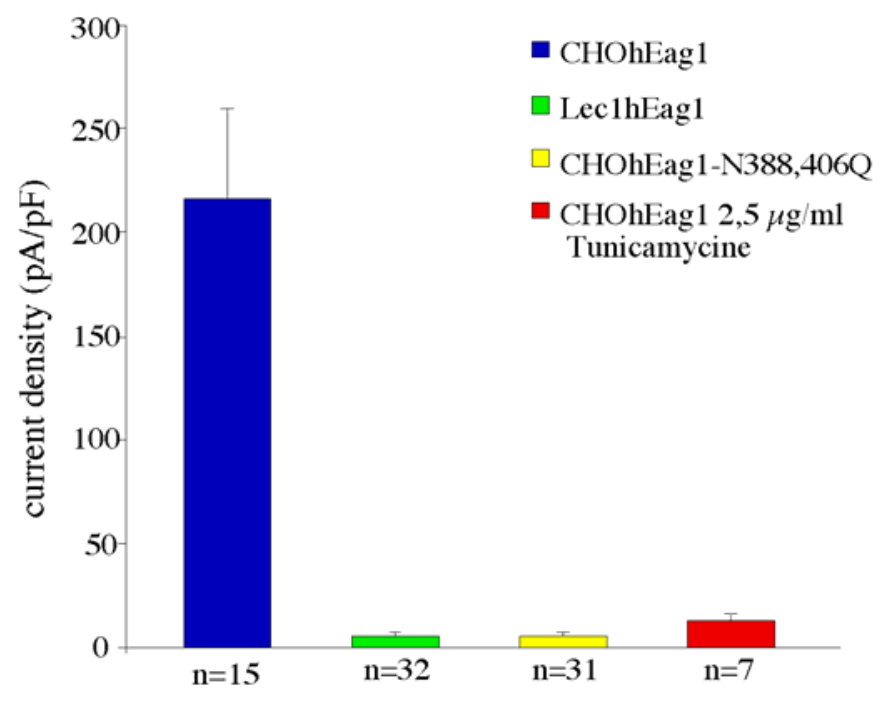

B

Fig. 29 Patch clamp recordings from CHO cells expressing non-glycosylated hEag1 channels.

A) Normalised current amplitudes recorded from:

- CHOhEag1

- CHOhEag1-N388,406Q (glycosylation mutant),

- CHOhEag1 after Tunicamycine treatment (2,5 $\mu \mathrm{g} / \mathrm{ml}$ for at least $15 \mathrm{~h})$,

- Lec1hEag1 (CHO-derived glycosylation deficient cell line)

The Cole-Moore effect was recorded using the protocol shown in inset. Note the strong slow-down in the activation kinetics in all three glycosylation deficient cell lines.

B) Statistical evaluation of the current density for wild type and unglycosylated hEag1 channels. Strong reduction of the current density was observed in all three glycosylation deficient cell lines. Currents were recorded by depolarisation from a holding potential of $-80 \mathrm{mV}$ to $+80 \mathrm{mV}$ 


\subsubsection{Identification of putative hEag1 binding partners by Yeast Two-Hybrid System}

To identify putative proteins binding to the C-terminus of hEag1 in vivo, MATCHMAKER

GAL4 Two-Hybrid system 3 was used (Fields and Song, 1989; Chien et al., 1991). This system is based on the activation of the four reporter genes when a bait and library proteins interact. A bait protein is expressed as a fusion protein to the GAL4 DNA binding domain and a library protein is expressed as a fusion protein to the GAL4 DNA activating domain. When brought into proximity, the complex binds to the GAL4 upstream activating sequences and activates transcription of HIS3, ADE2, lacZ and MEL1 genes.

ADE2 and HIS3 reporters provide a strong nutritional selection. So only this yeast, in which bait and pray proteins interact, are able to grow on adenine and histidine deficient media (-L/T/H/A). Additionally, MEL1 and lacZ encode $\alpha$-galactosidase and $\beta$-galactosidase, respectively, providing an additional tool for identification of interacting proteins by colour selection (positive colonies stain blue with X-gal).

\subsubsection{Cloning of the C-terminus of hEag1 into the pGBKT7 vector}

The analysis of the $\mathrm{Y} 2 \mathrm{H}$ interactions of membrane proteins is difficult because of their hydrophobic nature, thus, only the C-terminus of hEag1 was used to identify interacting proteins. A NcoI-BamHI fragment was cut out from the hEag1-pTracer clone and subcloned in frame into the pGBKT7 vector, which gave rise to the GAL4-hEag1CT fusion construct.

\subsubsection{Expression of the GAL4-hEag1CT fusion protein and transactivation test}

The GAL4-hEag1CT fusion construct was transformed into AH-109 yeast as described (2.2.7.1) and the expression was monitored by western blot.

In addition to the GAL4 DNA-binding domain, pGBKT7 vector contains also a c-myc epitope, which is located between the GAL4 domain and hEag1-CT in the fusion construct. 
On western blot both, the anti-Eag1 2413x polyclonal antibody and anti-myc monoclonal antibody, recognised strong signals only in AH-109 cells transformed with GAL4-hEag1CT, but not in the untransformed yeast (Fig. $30 \mathrm{~A}$ ). The size of the detected bands was in agreement with the calculated molecular weight for the GAL4-hEag1CT fusion protein, suggesting that the whole construct was properly expressed in AH-109 yeast and no posttranslational trimming or frame-shift occurred.

Moreover, to clarify whether the GAL4-hEag1CT itself has no transactivation properties (does not activate reporter genes), a series of controls, testing growth on different nutrientdeficient media containing additionally $\mathrm{X}$ - $\alpha$-galactose for blue colour selection, were performed in yeast.

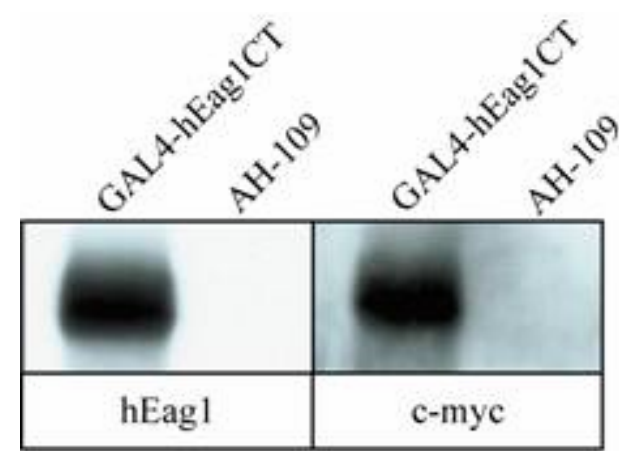

A

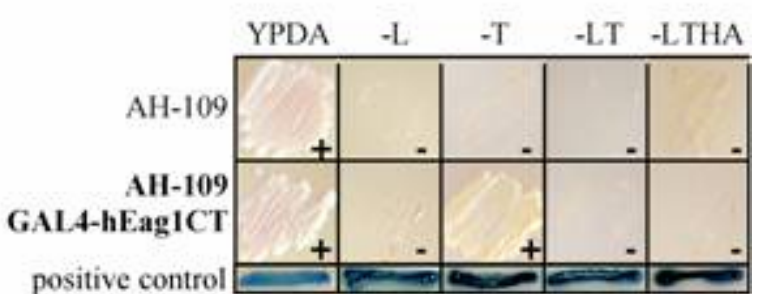

B

Fig. 30 Expression of the hEag1-CT bait protein in AH109 yeast

A) Whole cell lysates from AH109 transformed with pGBKT7-hEag1CT as well as untransformed AH108 yeast were separated by 7,5\% SDS-PAGE and immunobloted with either 2413x polyclonal antibody or anti c-Myc antibody. In both cases a strong signal corresponding to GAL4-hEag1CT bait protein was observed only in transformed yeast.

B) Phenotype confirmation of the AH109 and AH109 GAL4-hEag1CT expressing yeast. As expected untransformed yeast grows only on complete medium, whereas yeast transformed with the pGBKT7hEag1CT construct grows on the tryptophane deficient medium due to the expression vector.

Fig. 30 B shows that AH-109 GAL4-hEag1CT transformants grow only on the complete YPDA plates and on SD -Trp nutrient-selection plates (due to the pGBKT7 selection marker) but not on the other plates, although growth of positive controls was observed. No blue colonies were observed on the plates, although $\alpha$-galactosidase content was confirmed by positive controls. Moreover untransfected AH 109 were able to grow only on complete medium and did not turn blue by $\alpha$-galactosidase. 
This result verified the AH 109 phenotype (Ade, His, Leu and Trp auxotroph) and confirmed that the GAL4-hEag1CT fusion protein is not able to activate reporter genes in AH-109 yeast and has no toxic effects on the host strain, thus qualifying for a Two-Hybrid screen.

\subsubsection{Screening of a human brain cDNA library by yeast mating}

To identify proteins interacting with the C-terminus of hEag1 in vivo, the Pretransformed Human Brain MATCHMAKER cDNA Library was chosen. The titter of the library was calculated to $1,8 \times 10^{8} \mathrm{cfu} / \mathrm{ml}$. The library (in Y187 yeast) was screened using large scale mating protocols (mating efficiency was estimated to 8\%). Diploids containing putatively interacting proteins were selected on high stringency (SD, -LTHA) X- $\alpha$-galactose plates. In two independent screens, 30 colonies were able to grow on the nutrient-reduced minimal medium and demonstrated blue colour due to the galactosidase expression. Phenotypes were re-tested on fresh high stringency plates (SD -LTHA) containing X- $\alpha$-galactose and the library-plasmid DNA, encoding putative interaction partners of hEag1, was isolated as described (2.2.7.4).

\subsubsection{Analysis of the putative interaction partners of hEag1}

The most common false positives found in $\mathrm{Y} 2 \mathrm{H}$ screens include those, which activate reporter gene expression either because they are themselves able to induce transcriptional activation, or because they interact with the pGBKT7 vector and thus bring binding-domain and activation-domain into proximity. For this reason, the specificity of protein interactions was re-tested by yeast mating, as shown in Table 2. As a positive control AH109 pretransformed with pGBKT7-53, and Y187 pretransformed with pTD1-1 were mated, and as a negative control AH109 pGBKT7 GAL4-hEag1CT was mated with the Y187 pretransformed with pTD1-1. 
Four of the analysed 30 clones (Nr. 6, 9, 22 and 28) did not grow after transfection into the yeast. After 7 days none of the remaining 26 library clones showed autoactivation ability in both, nutrient selection and $\beta$-galactosidase assay.

\begin{tabular}{l|l|l|l}
$\begin{array}{l}\text { Y187 } \\
\text { transformed with: }\end{array}$ & $\begin{array}{l}\text { AH109 } \\
\text { transformed with: }\end{array}$ & $\beta$-gal test & growth on -LTHA \\
\hline library clone & $\begin{array}{l}\text { pGBKT7 } \\
\text { GAL4-hEag1CT }\end{array}$ & blue & yes \\
library clone & pGBKT7 & white & no \\
library clone & - & white & no
\end{tabular}

Table 4 Expected phenotype of diploides after control matings

Analysis of the diploids revealed that only a single clone (Nr. 1) showed strong positive interaction with bait protein but not with the expression vector in both nutrient selection and $\beta$-galactosidase assay.

Clones Nr. 8 and 15 showed also a positive interaction signals, but they were much weaker than the one observed for clone 1 (Fig. 31 A). However, for clones 8 and 15, specificity of the control transformations remains questionable and has to be further analysed.

Moreover, analysis of diploids containing the expression vector and the library clone revealed that most of the proteins identified as putative interaction partners were interacting with the GAL4-binding domain rather than with the bait protein. As shown in the example in Fig $31 \mathrm{C}$, most of these "false positives" showed even stronger interaction when coexpressed with the vector alone than with the bait protein.

As the clone Nr.1 showed strong positive interaction with hEag1CT only, we considered it as putative hEag1 interaction partner. Sequence analysis of the clone identified it as PIAS1 (protein inhibitor of activated STAT1). The library clone contained the almost complete ORF of the PIAS1 cDNA (2282 bp), except for the first 93 base pairs. PIAS1 is a nuclear protein. It was postulated that PIAS1 can block the DNA binding activity of STAT (transcription factor) and thus can inhibit STAT-mediated gene activation. 


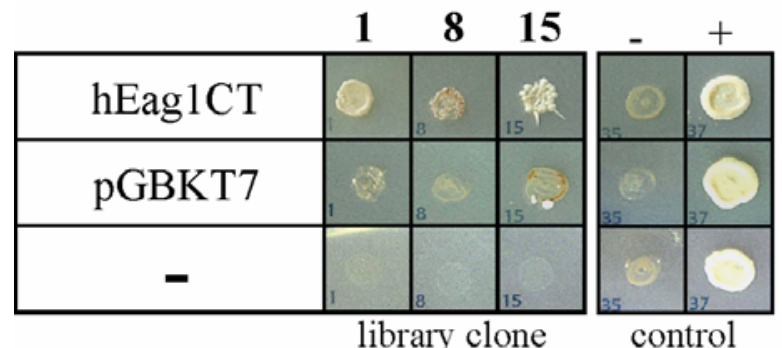

A

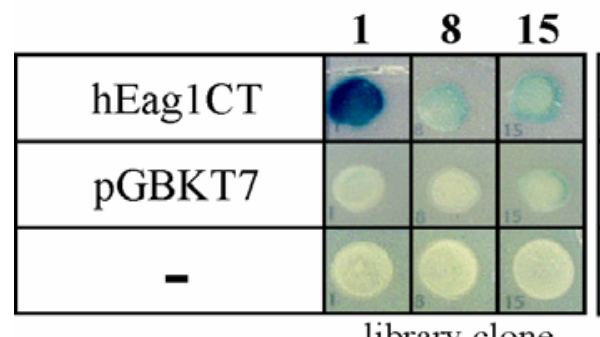

B

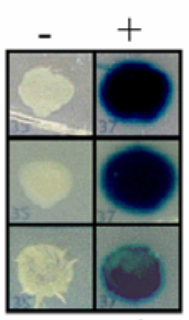

control

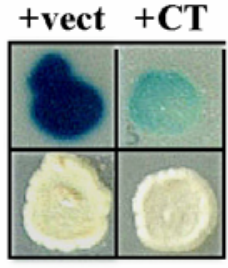

C

Fig. 31 Interactions of the hEag1 C-terminus in Yeast Two-Hybrid system

A) Nutrient selection and

B) $\beta$-galactosidase assay of the identified putative interaction partners of hEag1. Note that only one clone (Nr. 1) shows strong positive signal in both tests.

C) Example of the false positive clone (Nr. 5). Note that this clone shows much stronger blue staining in $\beta$-galactosidase assay when coexpressed with pGBKT7 then when expressed with bait protein.

There are several putative domains in the PIAS1 sequence, such as a nuclear receptor box (LXXLL), zinc binding motif, acidic domain and serine-threonine rich region (Shuai, 2000), but none of them gives a hint to a putative interaction between PIAS1 and hEag1.

Biochemical interactions become convincing only when they can be confirmed with an independent method for example overlay assay or co-precipitation. Moreover, for the $\mathrm{Y} 2 \mathrm{H}$, a good control is to test as well whether identified interactions occur in "both directions" i.e. after exchange of cloning vectors.

However, the independent proof of interaction of hEag1 with PIAS1, as well as with proteins encoded by cDNA clones Nr. 8 and Nr. 15 remains to be explored. 


\section{$4 \quad$ DISCUSSION}

$\mathrm{N}$-linked glycosylation is a common form of protein processing, which occurs on the integral membrane proteins and can profoundly affect protein expression, structure and function. The $\mathrm{N}$-linked oligosaccharide is assembled in a series of enzyme-catalysed reactions that start in the ER lumen and finish within Golgi membranes. Although N-linked glycosylation is a product of template-independent biosynthesis, sophisticated mechanisms exist to ensure that the correct structures are assembled.

It has been recognised for some time that glycosylation has great potential for carrying biological information. N-linked oligosaccharides are known to affect different physical properties of their proteins such as folding, stability, trafficking or assembly, as well as to influence functional properties like ligand binding or electrophysiological parameters. Although the precise physiological role is known for only a few glycoproteins, it is clear that the physiological properties of glycoproteins depend on both, the protein and the carbohydrate components.

Voltage gated potassium channels form a large family of membrane spanning proteins, many of which undergo N-linked glycosylation. Particular members possess not only very different oligosaccharide attachments, but the function of the N-linked sugars differs extremely between different channels.

The ether à go-go voltage-dependent potassium channel (Eag1) carries in its sequence as many as six putative glycosylation sites. In this work we used site directed mutagenesis and enzymatic treatments to study functional effects of N-linked glycosylation of human Eag1 expressed in heterologous systems. 


\subsection{Expression pattern of Eag1 protein in rat tissues}

Voltage-gated ion channels are hallmarks of excitable tissues. Although the expression of ion channels in these tissues is known for more than 50 years, only in the last few years have evolved an increasing number of reports suggesting functional roles of ion channels in nonexcitable tissues.

Ether á go-go (Eag1) is mainly expressed in the adult brain (human, mouse, rat). mRNA expression was also demonstrated in a few other tissues like human myoblasts and placenta (Occhiodoro et al., 1998; Pardo et al., 1999), human testis (Pardo, personal communication) or bovine retina (Frings et al., 1998).

In rat brain Eag1 expression is highest in granular cells of the dentate gyrus, hippocampus CA3 pyramidal cells, and in cerebellar granule cells, but it was also found in pituitary (Wulfsen et al., 2000) and spiral ligament (Lecain et al., 1999).

Additionally, Eag1 is expressed in highly proliferating non-excitable tissue, for example in human myoblasts at the onset of fusion (Occhiodoro et al., 1998). Cell lines of cancerous origin, for example cervical carcinoma (HeLa), particular lines from mammary gland tumours (Pardo et al., 1999), neuroblastoma (SH-SY5Y; Pardo et al., 1999, Meyer and Heinemann, 1999) or melanoma (Meyer et al., 1999; Gavrilova-Ruch et al., 2002) express high levels of Eag1. Furthermore, Eag1 is commonly found in different tumours but not in the corresponding healthy tissue (Pardo, personal communication).

Western blot analysis of tissue-specific expression of rat Eag1, with polyclonal antibodies directed against the C-terminal region of the protein revealed the expected signal in brain, but also in other organs: eye and testis. As the apparent molecular weight of the band detectable in eye, a part of the central nervous system, was similar to the brain-specific signal (see 3.1.1) and Eag1 mRNA was found before in bovine retina, it is very likely that it corresponds to the Eag1 protein expression in eye tissue.

In contrast, the protein detectable in rat testis was much smaller, migrating at about $58 \mathrm{kDa}$, instead of a broad band running at 120-130 kDa or the 2-band pattern detected in brain (see 3.1.1.1). The specificity of this signal was not further examined. However, as mentioned above, Pardo and colleagues (unpublished information) observed Eag1 expression also in human testis by real-time PCR. Thus, it is quite possible, that the polyclonal anti-Eag antibody detected a testis-specific isoform of the Eag1 protein. The different size of the band detected in rat testis could represent a tissue-specific splice-variant of Eag1 or a posttranslationally 
modified (for example truncated) protein. In could also be due to degradation, although protease inhibitors were included and all steps of the preparation were performed on ice. In any case, if specific, the band appears to include the C-terminal part of the Eag1, since the antibody used for the western blot analysis recognises this part of the protein. Nevertheless, crossreactivity of the antibody has to be considered also.

In testis the expression of different groups of ion channels, like ligand- and voltge-gated calcium channels and potassium channels is well documented. Ion channels were found in spermatogenic cells playing a role in different processes such as sperm maturation or fertilisation, but also in Leydig or Sertolli cells.

In the light of this variety the possible expression of an Eag1 protein in testis is not very surprising and should be further analysed.

The calculated molecular weight of a truncated putative channel $\alpha$-subunit, which could be formed by the S5, P, S6 and complete C-terminus of Eag1, is $68 \mathrm{kDa}$, which is higher than the observed signal. The calculated molecular weight of the C-terminal domain of rat Eag1 is $55 \mathrm{kDa}$, which is very similar to the apparent size of the signal observed in the western blot. Thus the question should be followed whether Eag1 can be functionally expressed in a truncated form or as a splice variant in rat testis.

\subsection{N-linked glycosylation of Eag1 potassium channels}

Many different ion channels posses one or more active glycosylation sites. Also voltage gated $\mathrm{K}^{+}$channel $\alpha$-subunits contain usually at least one consensus $\mathrm{N}$-linked glycosylation site within their primary amino acid sequence.

The accepted model of the voltage gated potassium channel structure postulated by Guy and Conti in 1990 (for details see Introduction and Fig. 1) describes six conserved transmembrane segments connected by intracellular or extracellular loops and with both the N-and C-terminal regions located intracellularly. Recently this model had to be slightly modified according to the first crystal data obtained for a six-transmembrane domain voltage-dependent potassium channel, KvAP (Jiang et al., 2003). The S3 helix now split into two individual helices, and the S4 domain is positioned perpendicularly (not, as believed, parallel) to the pore axis. Thus, considering also the analysis of hydrophobicity plots, it is unlikely that additional membrane spanning regions would show up, but it cannot be excluded. 
Sequence analysis of the hEag1 (splice variant a) protein sequence revealed the presence of six putative glycosylation sites, at asparagines 26, 238, 388, 406, 470, and 752. A schematic location of these amino acids in correlation to the putative domain structure of the hEag1 potassium channel is presented in Fig. 13. Two of the glycosylation motifs are located in the predicted intracellular N- and C-terminal domains, another two in putative transmembrane regions, and two in the extracellular loop between the S5 and P domains. Although only two of the putative $\mathrm{N}$-glycosylation sites are presumably extracellular, mutational analysis was directed to all six sites in order to exclude uncertainties in channel topology.

Putative N-glycosylation sites can be eliminated by single amino acid exchange in the AsnXaa-Ser/Thr consensus sequence. Although elimination of the Ser or Thr residue also abolishes the recognition site for the glycosylation machinery, usually the aspareagine as the actual N-glycosylation acceptor site is substituted by different amino acids.

For example Rho and colleagues (2000) chose serine for replacement of the asparagine in the analysis of bovine retinal cyclic nucleotide-gated (CNG) ion channel. However, in biochemical studies on Shaker potassium channels expressed in Xenopus oocytes (SantacruzToloza et al., 1994), three out of four consensus sites were individually eliminated by replacing the asparagine with glutamine. Similarly, Petreca and colleagues eliminated two putative glycosylation sites of HERG (human eag-related gene) potassium channel, replacing asparagine by glutamine (N598Q and N629Q).

For the site directed mutagenesis analysis of hEag1, also asparagines were chosen for the mutation and substituted with glutamines. Glutamine was used to minimise the influence of the amino acid exchange on the biochemical properties of hEag1, since asparagine and glutamine have comparable physical and chemical properties, such as weak polar side chains with a neutral, relatively unreactive amide group able to make hydrogen bonds of similar $\mathrm{pK}$ value.

Biochemical analysis of the hEag1 glycosylation mutants revealed that two of the motifs ${ }_{388}$ Asn-Asn-Ser 390 and ${ }_{406}$ Asn-Gly-Ser 408 participate in N-linked glycosylation of hEag1 in $\mathrm{CHO}$ cells (see 3.2.4). Both glycosylation sites are located in the predicted extracellular region between S5 and P, according to the Guy and Conti model of voltage gated potassium channels. Glycosylation was not detected on sites located in the hypothetical intracellular or transmembrane regions, in good agreement with the models of membrane topology (Guy and Conti, 1990; Jiang et al., 2003). 
The two hEag1-glycosylation-motifs are differentially conserved between different channelsubtypes and different species, as revealed by sequence alignment of different members of the eag family (see Fig. 32). The ${ }_{388}$ Asn-Asn-Ser 390 motif was found only in Eag1 channels of different mammals, but not in Deag. This sequence is completely conserved in all Eag1 channels known so far. No other eag family members posses this site.

The second motif, ${ }_{406}$ Asn-Gly-Ser 408 , was in contrast identified in all 15 examined proteinsequences, although several differences were found. The glycine at the second position in Eag1, Erg2 and Elk1 proteins is replaced by threonine in Eag2 by asparagine in Elk3 and by serine in almost all proteins except Deag1, chErg1 and hErg3, where a glutamine is found instead. Only in Drosophila Eag and Elk3 the third positions consist of threonine.

\begin{tabular}{|c|c|c|c|}
\hline hEAG1 & 371 & YSIGDYEIFDEDTKTIRNNSWLYQLAMDIGTPYQF - - - NGSGSGKWEGGPSKNSVYISSLYFTMTSLTSVGFGNIAPSTDIEKI & 451 \\
\hline bEag1 & 371 & YSIGDYEIFDEDTKTIRNNSWLYQLAMDIGTPYQF - - - NGSGSGKWEGGPSKNSVYISSLYFTMTSLTSVGFGNIAPSTDIEKI & 451 \\
\hline rEag1 & 371 & YSIGDYEIFDEDTKTIRNNSWLYQLALDIGTPYQF - - - NGSGSGKWEGGPSKNSVYISSLYFTMTSLTSVGFGNIAPSTDIEKI & 451 \\
\hline mEag1 & 398 & YSIGDYEIFDEDTKTIRNNSWLYQLALDIGTPYQF - - - NGSGSGKWEGGPSKNSVYISSLYFTMTSLTSVGFGNIAPSTDIEKI & 478 \\
\hline DEag & 405 & 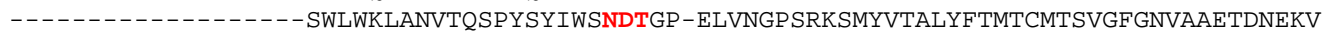 & 468 \\
\hline rEag2 & 368 & YSIGDYEVIDEVTNTIQIDSWLYQLALSIGTPYRY - - NTS - AGIWEGGPSKDSLYVSSLYFTMTSLTTIGFGNIAPTTDVEKM & 447 \\
\hline hEag2 & 368 & YSIGDYEVIDEVTNTIQIDSWLYQLALSIGTPYRY - - NTS - AGIWEGGPSKDSLYVSSLYFTMTSLTTIGFGNIAPTTDVEKM & 447 \\
\hline HERG1 & 569 & YAIGNMEQPHMDSRI - - - -GWLHNLGDQIGKPY - - - - NSSGLG - - - GPSIKDKYVTALYFTFSSLTSVGFGNVSPNTNSEKI & 639 \\
\hline rErg1 & 571 & YAIGNMEQPHMDSHI - - - -GWLHNLGDQIGKPY - - - - NSSGLG - - - GPSIKDKYVTALYFTFSSLTSVGFGNVSPNTNSEKI & 641 \\
\hline mErg1 & 571 & YAIGNMEQPHMDSHI - - - -GWLHNLGDQIGKPY - - - - NSSGLG - - - GPSIKDKYVTALYFTFSSLTSVGFGNVSPNTNSEKI & 641 \\
\hline doErg1 & 568 & YAIGNMEQPHMDSRI - - - -GWLHNLGDQIGKPY - - - - NSSGLG - - - GPSIKDKYVTALYFTFSSLTSVGFGNVSPNTNSEKI & 638 \\
\hline raErg1 & 571 & YAIGNMEQPHMDSRI - - - - GWLHNLGDQMGKPY - - - - NSSGLG - - - GPSIKDKYVTGLYFTFSSLTSVGFGNVSPNTNSEKI & 641 \\
\hline chErg1 & 288 & 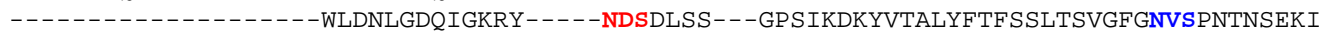 & 343 \\
\hline hErg2 & 420 & YAIGNVERPYLEHKI - - - GWLDSLGVQLGKRY - - - - NGSDPAS - - GPSVQDKYVTALYFTFSSLTSVGFGNVSPNTNSEKV & 491 \\
\hline rErg2 & 420 & YAIGNVERPYLEPKI - - - -GWLDSLGAQLGKQY - - - - NGSDPAS - - -GPSVQDEKYVTALYFTFSSLTSVGFGNVSPNTNSEKV & 491 \\
\hline hERG3 & 587 & 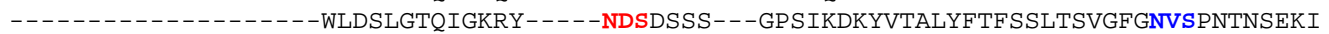 & 642 \\
\hline hELK1 & 400 & 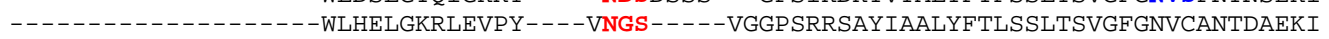 & 454 \\
\hline rElk1 & 401 & 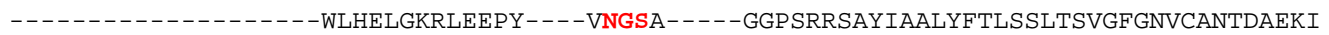 & 455 \\
\hline hElk2 & 398 & 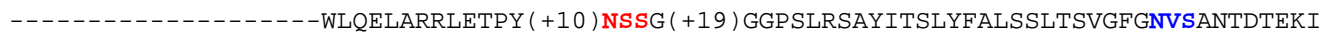 & 480 \\
\hline mElk2 & 398 & 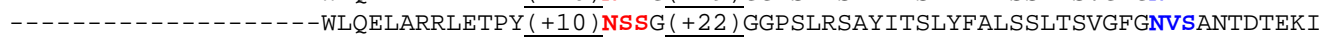 & 483 \\
\hline mElk2 & 398 & 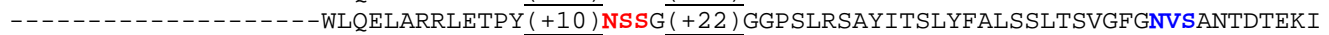 & 483 \\
\hline hElk3 & 380 & - - - - MEREDNSLLKWEVGWLHELGKRLESPYYG - - NNTL- - - GGPSIRSAYIAALYFTLSSLTSVGFGNVSANTDAEKI & 449 \\
\hline rElk3 & 375 & YVIG - KMEREDNSLLKWEVGWLHELGKRLESPYYG - - NNTL - - - - GGPSIRSAYIAALYFTLSSLTSVGFGNVSANTDAEKI & 449 \\
\hline mElk3 & 149 & YVIG-KMEREDNSLLKWEVGWLHELGKRLESPYYG - - NNTL - - - - GGPSIRSAYIAALYFTLSSLTSVGFGNVSANTDAEKI & 223 \\
\hline
\end{tabular}

Fig. 32 Multiple sequence alignment of selected members of the eag voltage-gated potassium channel family

Amino acid sequences of 24 potassium channels (Eag, Erg and Elk channels) with highest homology to the extracellular loop between S5 and S6 (including P region) of hEag1 were identified by BLAST search and aligned with CustalW. The following species are included: h-human, b-bovine, r-rat, m-mouse, do-dog, ra-rabbit, ch-chicken and D-Drosophila.

The sequence of the hEag1 protein is shown in bold.

Homologues of both hEag1 N-glycosylation consensus sites are shown in red and bold. The first glycosylation motif is conserved only through the Eag1 family, whereas the second one was found in all analysed sequences. An additional glycosylation site found in the Erg family is shown in blue and bold.

The impact of the second amino acid in the N-glycosylation sequon on the efficiency of the glycosylation process was analysed by Shakin-Eshleman and colleagues (1996). 20 different variants of a model glycoprotein, presenting each of the common amino acids at position $\mathrm{X}$ of an N-X-S motif were examined using a rabbit reticulocyte translation system and the efficiency of core glycosylation was quantified. For this, the density of bands representing glycosylated $(\mathrm{G})$ and nonglycosylated $(\mathrm{N})$ proteins were quantified for each variant and 
glycosylation efficiency was calculated: $\mathrm{G} /(\mathrm{N}+\mathrm{G}) \times 100 \%$. These studies showed that the presence of proline at the position $\mathrm{X}$ completely blocks core glycosylation, whereas tryptophan, aspartic acid, glutamic acid and leucin are associated with inefficient core glycosylation. According to these results, a poor glycosylation of the second motif would be expected in chErg1 hErg3 and Deag channels, where aspartic acid is in the second position. According to Shakin-Eshleman et al. (1996), is the efficiency of N-linked glycosylation of hEag1 on the ${ }_{388}$ Asn-Asn-Ser 390 sequon about $70 \%$ and on the ${ }_{406}$ Asn-Gly-Ser ${ }_{408}$ sequon about $90 \%$. All other amino acids found at the second position in the glycosylation motifs of eag family members, should have a N-glycosylation efficiency between $70 \%$ and $100 \%$.

In all listed Erg proteins (Erg1, 2 and 3) as well as in Elk2 and Elk3 (but not in Elk1) a second, conserved motif (at the position 629-631 in HERG1) is present. This site was previously reported to be glycosylated in HERG1 (Petrecca et al., 1999). The valine at position $\mathrm{X}$ of this sequon is highly conserved and should be glycosylated with $80 \%$ efficiency according to Shakin-Eshleman studies (1996).

\subsection{Model for N-linked glycosylation of human ether á go-go potassium channel}

Glycosylation of voltage-gated ion channels has often been reported. In most cases, one or two oligosaccharide attachments are described, which vary in size, composition and location on the protein between individual channels and/or different channel families.

The presence of N-linked oligosaccharides on the protein can be monitored by western-blot, where a higher than the predicted molecular weight or a banded pattern are good indications for this type of modification.

For HERG a member of the eag potassium channels family, Zhou and colleagues (1998 A) showed, that a two-band migration pattern $(155$ and $135 \mathrm{kDa})$ on the western blots can be attributed to $\mathrm{N}$-linked glycosylation. N-glycosidase F treatment converted both proteins to lower molecular weights, but not into a single band, suggesting, that additional posttranslational modifications might occur. The same group later showed (Zhou et al., 1998 B), that the upper band is resistant to Endo $\mathrm{H}$ digestion, and suggested that the $155-\mathrm{kDa}$ band is the complex-glycosylated, mature form of the HERG channel and the 135-kDa band is a coreglycosylated precursor form of the HERG channel. Site-directed mutagenesis of HERG, done 
by Petreca and colleagues (1999) revealed presence of two active glycosylation sites (N598 and N629; compare with Fig. 32). Neither single (N598Q or N629Q) nor double mutant proteins were glycosylated in HEK 293 cells, indicating that both sites must be available for N-linked glycosylation to occur. In contrast, Gong and colleagues (2002) showed that the N629Q HERG mutant protein undergoes core glycosylation in the ER; thus, documenting that also a single site can be glycosylated in HERG channels.

Interestingly, a mutation away from the glycosylation motif was shown to affect N-linked glycosylation of HERG. Zhou (1998) demonstrated that the spontaneous mutation Y611H (found in a LQT patient) located 13 amino acids upstream (first motif) and 18 amino acids downstream (second motif) from the asparagine residues that act as oligosaccharide acceptors, completely abolished N-glycosylation of HERG, probably by affecting the structure of the protein.

Also for Shaker, a banded migration pattern was observed in western blots (Santacruz-Toloza et al., 1994). Three bands were detected when expressed in insect Sf9 cells, and two bands when expressed in Xenopus oocytes. Tunicamycine treatment as well as endoglycosidase digestion revealed that both upper bands carry oligosaccharide attachments whereas the lower one detected in Sf9 cells represents the non-modified Shaker protein. Santacruz-Toloza and colleagues (1994) mutated three out of four consensus sites for N-linked glycosylation in Shaker. The two identified N-glycosylated asparagines are located in the first extracellular loop between the S1 and S2 domains, whose mutations N259Q and N263Q, only partially reduced the molecular weight of the upper band. The resulting protein was still sensitive to PNGaseF, demonstrating that one site is sufficient for the N-linked glycosylation (although not complete) of Shaker potassium channels. The double mutant contains no N-linked oligosaccharide since the migration of this protein is unaltered by treatment with $\mathrm{N}$ glycosidase F.

$\mathrm{P} 2 \mathrm{X} 1$ receptors carry as many as four glycosylation sites in the external loop, but only $\mathrm{Asn}_{300}$ acquires complex carbohydrates (Rettinger et al., 2000). In contrast, cyclic nucleotide-gated (CNG) ion channels contain only a single active glycosylation site (N327) in the external loop between the S5 and P regions (Rho et al., 2000).

Although N-linked glycosylation is a common modification of plasma-membrane proteins, not all ion channels undergo glycosylation. For example, Kv1.6 lacks consensus N-glycosylation sites in the predicted extracellular domains (Shi et al., 1999). In accordance with this did PNGase digestion of Kv2.1, another member of voltage-gated $\mathrm{K}+$ channels family, not change the apparent molecular weight. This suggests, that the single consensus site (between 
S3 and S4) is not actively glycosylated in rat brain, although other members of the family (Kv1.1, Kv1.2 and Kv1.4) can undergo glycosylation (Shi et al., 1999).

In this study immuno-blot analysis of hEag1 revealed that the protein exists in two molecular mass species: $\approx 110$ and $\approx 130 \mathrm{kDa}$ (see Fig. 11 ), which is compatible to HERG. Both detected hEag1 proteins carried N-linked oligosaccharide attachments, as the band were shifted to lower molecular weight upon enzymatic deglycosylation with PNGase F (3.2.2).

Subsequently, only the $110 \mathrm{kDa}$ band was sensitive to Endoglycosidase $\mathrm{H}$ treatment, arguing for two populations of carbohydrates being attached to hEag1 in CHO cells:

- the core-oligosaccharide, sensitive to Endo H and represented by the $110 \mathrm{kDa}$ band, and

- the complex-oligosaccharide, Endo H resistant and corresponding to the $130 \mathrm{kDa}$ band.

Mutational analysis of the six putative glycosylation sites revealed that two residues, $\mathrm{Asn}_{388}$ and $\mathrm{Asn}_{406}$ are active acceptors of $\mathrm{N}$-linked oligosaccharides, each of them being responsible for one type of oligosaccharide attachment. $\mathrm{Asn}_{388}$ was shown to carry small coreoligosaccharide only, $\mathrm{Asn}_{406}$ in contrast a large complex-sugar structure. Both glycosylation sites of hEag1 are modified independently from each other. Thus, mutation of the $\mathrm{Asn}_{388}$ site did not affect glycosylation of the Asn 406 site and vice versa.

Furthermore, the Concanavalin A binding assay (see 3.2.7) showed that both glycosylation sites are (at least for some population of channels) occupied concomitantly and that the different forms of glycosylated hEag1 are present in cells. Concanavalin A recognises only core-oligosaccharides attached to hEag1, since it binds to hEag1-N406Q but not to hEag1-N388Q mutant proteins. In non-mutated hEag1, however, two protein populations were detected by Concanavalin A (schematically represented in Fig. 33).

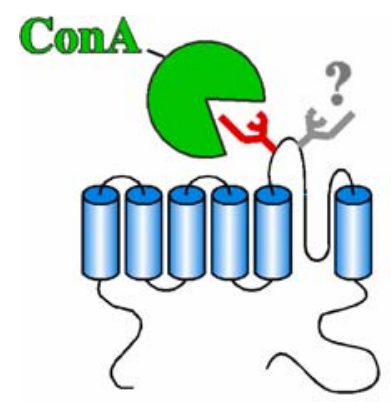

A

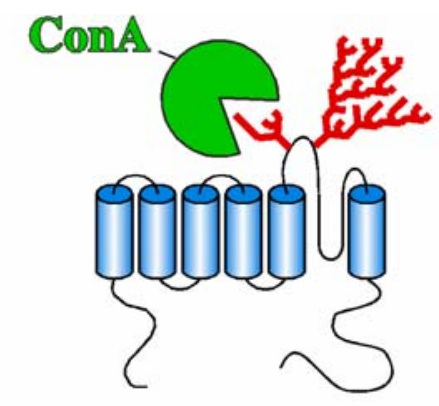

B

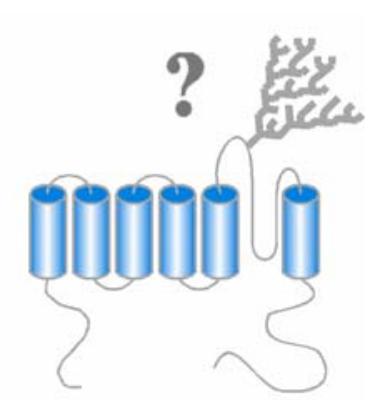

C

Fig. 33 Differential glycosylation of the hEag1 protein in CHO cells according to the Concanavalin A detection pattern 
The first population migrates at level compatible with the core-glycosylated mutant $(\approx 110 \mathrm{kDa})$ and probably represents the newly synthesised, incompletely processed glycoprotein. This population is schematically represented in Fig. 33 A. Because of the Endo $\mathrm{H}$ sensitivity of the E-110 band we assumed that it corresponds to the core-glycosylated protein. Because the $\mathrm{N} 388 \mathrm{Q}$ mutant lacks the E-110 band, we assumed that the band represents the hEag1 protein glycosylated on $\mathrm{Asn}_{388}$. It cannot be excluded that in the nonmutated protein both glycosylation sites can be occupied by small molecular mass carboxyhydrates, resulting in the E-110 band (Fig. 33 A). A core glycosylation on the Asn 406 and a transient core-glycosylated $\mathrm{Asn}_{388}$ could co-exist at least for a short time. On the other hand, transient core glycosylation on $\mathrm{Asn}_{388}$ seems to exist only for a very short time, since in the N388Q mutant a core glycosylated form of the hEag1 protein was not detectable.

The calculated molecular mass of the oligosaccharide scaffold directly after transfer onto a nascent protein, consisting of two GlcNAc nine mannoses and three glucoses, is only $2.368 \mathrm{Da}$ (GlycanMass at the ExPASy). Thus, due to the resolution of the SDS-PAGE gel, the question, whether a hEag1 protein with a single or double core-oligosaccharide attachment corresponds to the E-110 band, cannot be answered.

The second population recognised by ConA (Fig. 33 B) migrates at higher molecular weights $(\approx 130 \mathrm{kDa})$, at a level compatible with a complex-glycosylated (EndoH insensitive) form of hEag1. This population is likely to contain both oligosaccharide attachments: the core-one at $\mathrm{Asn}_{388}$, because it is recognised by ConA, and the complex-one at $\mathrm{Asn}_{406}$, because of the high molecular weight of the detected band.

Existence of an additional isoform with only the single, complex-oligosaccharide attachment (Fig. 33 C), which is not recognisable by ConA, was not further analysed, but it cannot be excluded since in the N388Q mutant complex glycosylation of $\mathrm{Asn}_{406}$ is not affected in a detectable manner (see 3.2.4).

Additionally, the isoforms of the hEag1 glycoprotein are differentially sorted into cellular fractions as shown by differential centrifugation. In these experiments (3.2.8.1), the E-130 isoform was predominantly detectable in P-100 and the E-110 isoform in P-1 fractions. This was not so significant in fractionation experiments on sucrose gradients, but also here both isoforms were differentially distributed through the fractions. The E-110 isoform was predominantly found in fractions containing also the reticular marker calreticulin, whereas E-130 isoform did not show such a concentrated distribution. 
The differential distribution could be because both isoforms could have different destiny and thus are directed to different cellular compartments.

\subsection{Effects of $\mathrm{N}$-linked glycosylation on the intracellular transport of hEag1}

It is generally believed that N-linked glycosylation may influence different stages of protein biosynthesis and transport, such as: protein folding and subsequent export from the ER, protein stability (mostly by protection from proteolytic degradation) or intracellular trafficking. However, the role of carbohydrate moieties varies among different membrane proteins, including ion channels and membrane receptors.

In the MCHR1 receptor, disruption of all three glycosylation sites impaired the expression at the cell surface as well as receptor activity (Saito et al., 2003). Patch clamp analysis, surface biotinylation, and immunofluorescence microscopy of SUR1 (sulfonylurea receptor), coexpressed with Kir6.2, demonstrated a significant decrease of SUR1 surface expression and prominent retention within the ER (Conti et al., 2002). For unglycosylated TSHR (thyreotropin receptor) inefficient expression on the cell surface was shown by expression in glycosylation deficient cell lines (Lec1 and Lec2 CHO mutants) as well as by Tunicamycin treatment in CHO cells stably expressing TSHR (He et al., 2002).

Importance of the N-linked glycosylation for the cell surface expression has also been reported for some ligand-gated channels including ATP purinergic receptors P2X1 and P2X2 (Rettinger et al., 2000; Torres et al., 1998).

Glycosidase digestion studies and immunocytochemical analysis of ENaC (epithelial sodium channel) show that channel subunits are retarded in the ER and pre-Golgi, whereas only a small fraction is inserted into the plasma membrane (Dijkink et al., 2002).

Studies on the Shaker potassium channel revealed that N-linked glycosylation strongly increases cell membrane expression of the channel expressed in mammalian cells, although it is not absolutely required (Khanna et al., 2001, Moreno et al., 2002, de Souza et al., 2002). By confocal microscopy Khanna and colleagues showed that a significant fraction of the unglycosylated Shaker mutant-protein (N259Q,N263Q) was retained intracellularly and its distribution overlapped with ER markers, although no such overlap was seen for the wild type protein when expressed in HEK293T cells. Moreover, cell surface biotinylation experiments 
showed that expression at the cell surface was significantly higher $(\approx 7$-fold) for the glycosylated than for the unglycosylated Shaker channel. Additionally, using quantitative imaging, de Souza and colleagues (2002) observed, that unglycosylated mutants traffic more slowly from the ER to the Golgi than wild type channels, which may contribute to the slower delivery of the mutant to the cell surface.

For HERG, a close relative of the hEag1 potassium channel, slightly contradictory reports have been presented. Zhou and colleagues (1998 B) showed that mutation Y611H, known to affect N-linked glycosylation of HERG, resulted in channel retention in the ER. Additionally Petrecca and colleagues (1999) described, that Tunicamycin blocked normal surface membrane expression of HERG-GFP fusion protein in HEK293 cells. In contrast, Gong and colleagues (2002) presented that N-linked glycosylation is not required for the cell surface expression of functional HERG channels (The same group reported also that N629 is not used for glycosylation, but mutation of this site (N629Q) affected protein trafficking and caused intracellular retention.

hEag1 as a voltage gated potassium channel, is presumed to be a plasma membrane protein. In disagreement with this, hEag1 showed diffuse intracellular localisation with no, or very slight membrane staining, when expressed in $\mathrm{CHO}$ cells. This cannot be simply explained by putative processing or transport difficulties due to expression in a heterologous system, since similar expression pattern could be observed by other members of our group in native hippocampal neurons, as shown in Fig. 34.

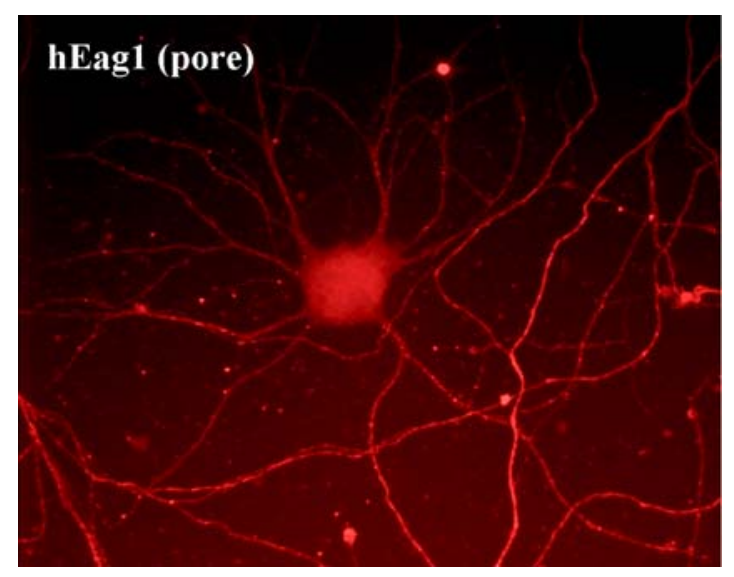

Fig. 34. Expression of hEag1 in hippocampal neurons (Rubio \& Sánchez, personal communication) Neurons were stained with mAb-62 antibody directed against the pore of hEag1. 
Detailed analysis of the hEag1 sequence revealed the presence of a reticulum retention signal, a RKR (RXR) motif, which was first described in subunits of ATP sensitive potassium channels (Zerangue et al., 1999). As described for the K(ATP) channel $\alpha$ - (Kir6.1/2) and $\beta$ (SUR1) subunits, the RKR signal helps, in most cases, to retain individual subunits and incomplete oligomers in the ER until they are masked by oligomeric assembly. A similar arginine based motif, RGR, was found also in HERG channels. Here it is believed, that the retention signal is masked by the C-terminal region of properly folded HERG channels. When the C-terminus is truncated, the RGR signal becomes exposed and causes trafficking impairment (Kupershmidt et al., 2002). As already mentioned, also HERG does not show an exclusive membrane staining when expressed in mammalian cells (Zhou et al., 1998 B).

Experimental evidence is presented for the influence of $\mathrm{N}$-linked glycosylation on intracellular trafficking of hEag1 potassium channels. Expression of hEag1 in glycosylationdeficient Lec1 cells, or Tunicamycin treatment of $\mathrm{CHO}$ cells showed that the unglycosylated hEag1 protein localises to complex perinuclear structures. In contrast, wild-type CHO cells transfected with hEag1 showed a disperse distribution throughout the whole cell and cell processes.

Immunofluorescence studies on glycosylation deficient mutants with single (N388Q and $\mathrm{N} 406 \mathrm{Q})$ or both $(\mathrm{N} 388,406 \mathrm{Q}) \mathrm{N}$-glycosylation motifs affected, revealed that proper glycosylation at asparagine 406 is necessary for intracellular trafficking of hEag1. Both N406Q and N388,406Q mutants showed perinuclear accumulation, similar to the pattern observed in glycosylation deficient cells. In contrast, the distribution pattern of the N388Q mutant was similar to the one observed with wild-type hEag1, although in some cells an increased perinuclear localisation was also detected.

These results were confirmed by functional characterisation of hEag1 and mutant channels in Xenopus oocytes. Mutation at asparagine 406, abolishing the complex glycosylation of hEag1, resulted in a dramatic reduction (about 6.5-fold) of the average peak current amplitude in comparison to wild-type channels, when equal amounts of RNA were injected. This can be explained by several factors:

- inefficient translation.

- reduced voltage-sensitivity of the mutant channel

- altered single channel properties in the N406Q mutant

- decreased number of channels in the membrane 
N406Q mutant channels indeed showed reduced voltage sensitivity, but the $25 \mathrm{mV}$ difference in the activation curve was not large enough to explain observed reduction in current density. The possible reduction of the current amplitude due to the $25 \mathrm{mV}$ shift in voltage sensitivity would be $20-30 \%$ and not, as observed more than 6 fold.

The hypothesis that the reduced current amplitude of N406Q mutant channels is caused by a lower number of channels in the membrane is in agreement with the immunocytochemical observations showing that complex glycosylation at asparagine 406 is necessary for the normal distribution of hEag1 in $\mathrm{CHO}$ cells. Thus, the hypothesis is favoured that the trafficking is affected, in explaining the reduced current amplitude of N406Q mutant channels.

Based on colocalisation experiments, where hEagl and mutants lacking single Nglycosylation sites (N388Q and N406Q) expressed in CHO cells were co-detected with markers for different trafficking organelles, it is suggested that the glycosylation deficient mutants accumulates in a part of the endoplasmatic reticulum. The N406Q mutant, lacking the site of complex-oligosaccharide attachment, showed increased colocalisation with reticular markers (TRAP $\alpha$, calreticulin).

Endoplasmatic reticulum provides an environment that is optimised for protein folding and maturation, some posttranslational modifications (e.g. N-glycosylation) and oligomeric assembly. Additionally it provides so-called 'quality control systems', particularly well characterised in the case of glycoproteins. Briefly, after the cotranslational oligosaccharide transfer, the nascent glycoprotein is bound by chaperones calnexin/calreticulin as well as by ERp57 (associated thiol oxidoreductase). The subsequent cycle of unbinding and rebinding to these chaperones supports correct folding of glycoproteins. Only properly folded proteins exit this cycle and are further exported to the Golgi where the carbohydrates are further trimmed and modified, en route to the plasma membrane (Ellgaard et al., 1999; Ellgaard et al., 2003). Thus it can be possible that hEagl lacking the complex-oligosaccharide residue is not properly folded and thus not easily exported out of the endoplasmatic reticulum.

Some of the ER-retained proteins are quite stable and can accumulate in distant regions of the ER although prolonged retention of misfolded and incompletely folded proteins leads, in most cases, to their degradation in a process called ERAD (endoplasmatic reticulum associated degradation (Bonifacino and Weissman 1998). This is linked to the export of proteins to the cytosol and proteolytic degradation by the ubiquitin proteasome system.

The observed perinuclear structure could thus correspond to the distant part of the ER accumulating the putatively misfolded non-glycosylated (or not complex-glycosylated) hEag1 
protein. On the other hand it could also represent a place transient localisation of hEag1 on the way to proteolytic degradation.

Additional experiments to quantify the arrival of hEag1 and mutant channels to the plasmamembrane, such as cell surface biotinylation and Proteinase K digestion (data not shown), failed. This can be explained by the fact that a significant fraction of hEag1 localises intracellularly in $\mathrm{CHO}$ cells (see 3.2.9), making the membrane fraction undetectable by standard biochemical methods, since it represents only a few percent of the total cellular pool of hEag1. Thus, although it is known that at least some of the hEag1 and mutant channels are able to reach the plasma membrane (since small hEag1 currents can be measured), a quantification of the protein expressed at the cell surface is not possible.

\subsection{N-linked glycosylation and functional properties of hEag1}

Thinking about N-linked glycosylation, one has to imagine the addition of the extensive, complex glycan composition to the synthesised protein. Just by its size, the oligosaccharide can easily affect structure, transport or biochemical properties, but it can also alter the function of plasma membrane proteins, such as receptors or ion channels.

For receptors, the most common functional effect of N-glycosylation involves ligand binding ability, although not all receptors are dependent on saccharide attachment. Hence, for example, N-glycosylation of CRFR1 (corticotropin-releasing factor type 1 receptor) is essential for ligand binding (Assil et al., 2001). In contrast, for the gonadotropin-releasing hormone receptor, glycosylation was described to have no effect on ligand binding (Davidson et al., 1995).

Many voltage-gated ion channels are heavily glycosylated, and the contribution of carbohydrates to the molecular weight of the mature protein can reach $15-40 \%$. Thus, carbohydrate moieties often significantly contribute to the functional properties of channels.

The effects of N-glycosylation on ion channel activity vary enormously. For example, it has no significant effect on current density or voltage dependence of Shaker (Santacruz-Toloza et al., 1994). In contrast, in ROMK1 (inwardly rectifying $\mathrm{K}+$ channel), loss of glycosylation produces a great reduction of whole-cell currents, caused by a dramatic decrease in open probability (Schwalbe et al., 1995). In Kv1.1 channels, loss of glycosylation gives rise 
to a shift of the voltage dependence of activation to more positive voltages, and slow down of activation kinetics (Thornhill et al., 1996). For $I_{\mathrm{sK}}$ channels, besides a similar shift of the voltage dependence of the activation and slow down of the activation kinetics, strong effects on $\mathrm{pH}$ sensitivity were observed, when they co-assembled with unglycosylated minK subunits (Freeman et al., 2000).

All these data indicate that N-linked glycosylation may play a pivotal role in the modulation of ion channels from different subfamilies.

In agreement with these observations, the results presented here show that the functional properties of hEagl channels also depend on N-linked glycosylation. The loss of glycosylation on $\mathrm{Asn}_{406}$ causes a strong reduction of the hEag1 current density in both Xenopus oocytes and in mammalian cells, and identifies trafficking problems as a determinant factor (discussed in 4.4).

Moreover, the complex N-glycosylation on $\mathrm{Asn}_{406}$ may contribute to the modulation of the voltage dependence of $\mathrm{hEag} 1$ channels. The voltage at the half maximal activation $\left(\mathrm{V}_{1 / 2}\right)$ was shifted $25 \mathrm{mV}$ towards more positive potentials in N406Q mutant channels in comparison to hEag1. Furthermore loss of glycosylation caused by different factors (mutation N406Q, N388,406Q, expression in Lec1 cells or Tunicamycin treatment) significantly slowed down the kinetics of hEag1 activation.

It is noticeable that the conserved site for complex $\mathrm{N}$-glycosylation $\left(\mathrm{Asn}_{406}\right)$ is located in the outer vestibule of the channel near the P-region. Since the vestibules of ion channels are believed to influence the permeation of ions, and the P-region mainly determines the characteristics of permeation, glycosylation near the pore mouth can affect the channel function by structural interference with the pore region. Additionally, N-linked glycosylation can generate surface charge effects as reported for the desialidation (Bennett et al., 1997).

It is known, that a number of voltage gated $\mathrm{Na}+$ (Bennett 2002) and some $\mathrm{K}^{+}$channels (Thornhill et al., 1996) can bear large amounts of posttranslationally attached carbohydrates consisting mostly of the negatively charged sialic acid. Contribution of sialic acid to the voltage dependence and channel gating is well explored for voltage gated $\mathrm{Na}^{+}$channels (Bennett et al., 1997; Bennett 2002). It is known that the actual electric field experienced by a channel voltage-sensing mechanism is an important component of gating. Fixed negative charges on the external membrane surface, which can be provided by the sialic acid attachment, can electrostatically reduce the resting transmembrane electric field sensed by 
gating elements. Thus, for $\mathrm{Na}^{+}$channels, desialidation causes the channels to activate at smaller depolarisations.

A similar mechanism could also explain the functional effects of N-linked glycosylation observed in hEag1 channels. Addition of negatively charged oligosaccharides, such as sialic acid, could cause a rearrangement of the net charge detectable by the voltage sensor of hEag1 and thus modify voltage dependence and kinetics of the current.

Each cell type expresses its unique panel of potassium channels as homo- and/or as heteromultimers, generating a broad molecular and functional diversity of channel population. $\alpha$-subunits assemble early during biosynthesis in the ER giving rise to a basic pool of variation. Apart from this, potassium channels can generate additional functional diversity by controlling the manner in which the $\alpha$-subunits can interact with other cellular proteins, such as G proteins (Wickman and Clapham 1995) SH3 or PSD-95 (Scannevin et al., 1997). Functional diversity can be further increased by interactions with $\beta$-subunits modulating properties of the ion channels in two ways: modulating cell-surface expression of $\alpha$-subunits (Shi et al., 1996; Nagaya and Papazian 1997), or modulating gating properties of the channel (Heinemann et al., 1994; Rettig et al., 1994). Moreover, electrically silent modulatory alpha subunits are known to contribute to the molecular and functional diversity of ion channels (Hugnot et al., 1996; Castellano et al., 1997; Kerschensteiner et al., 1999).

Beyond this, channel properties can be also modulated by reversible modifications such as phosphorylation/dephosphorylation (Antz et al., 1999).

So far, a few proteins such as hyperkinetic in Drosophila (Wilson et al., 1998), epsin and KCR1 in rat (Piros et al., 1999; Hoshi et al., 1998) and calmodulin in hEag1 expressing Xenopus oocytes (Schonherr et al., 2000) have been found to interact with Eag1 channels. Hyperkinetic dramatically increases the amplitude of DEag currents and also affects gating and modulation by progesterone (Wilson et al., 1998). Calmodulin is known to bind to the Cterminal motif of human ether á go-go potassium channel and to inhibit Eag1 currents in a $\mathrm{Ca}^{2+}$ dependent manner (Schonherr et al., 2000). Epsin, a protein involved in endocytosis (Chen et al., 1998), was found to interact with C-terminus of hEag1 in overlay assays, but the functional implications of its binding still remain unknown (Piros et al., 1999). Similarly, $\mathrm{KCR} 1$ interacts with the C-terminus of rat Eag1. It is believed to function as a regulatory element, although its function is not well understood (Hoshi et al., 1998). 
As shown, N-linked glycosylation can strongly modify properties of the human ether á go-go voltage dependent potassium channel, and thus can represent an additional regulatory mechanism controlling channel function, e.g. by fine modulating voltage dependence and gating kinetics of the Eag1 channels.

\subsection{Does N-linked glycosylation affect the stability of hEag1?}

Glycosylation is also known to protect proteins from degradation and to increase their stability. Good examples for the protective function of $\mathrm{N}$-linked glycosylation are oligosaccharides, which protect a subset of lysosomal membrane proteins from proteolytic digestion in intact cells (Kundra and Kornfeld, 1999).

There are several explanations for the way in which N-linked glycosylation can impair protein degradation. First, simply by means of their size, large oligosaccharides can cover the protein surface, making the recognition sites inaccessible for proteases. Alternatively can calnexin and calreticulin, by binding to newly glycosylated proteins in the ER protect them from retrotranslocation and proteasomal degradation. Moreover, loss of glycosylation can simply cause misfolding and thus produce directing of unglycosylated proteins to proteasomal degradation.

Glycosylation is also known to increase the stability of various ion channels. Khanna and colleagues (2001) showed that the unglycosylated Shaker protein is unstable and rapidly degraded by cytoplasmatic proteasomes, although no effects on folding and assembly were observed. Also unglycosylated HERG channels showed faster turnover rates compared with glycosylated channel proteins (Gong et al., 2002).

Here, several indications supporting the idea that N-linked glycosylation may increase the stability of hEag1 are presented:

- in the N388Q mutant the lower (E-110) band, corresponding to unglycosylated hEag1, was frequently missing on western blots

- the double mutant N388,406Q was very difficult to handle and was frequently missing on western-blots

- detection of the $\mathrm{N} 388,406 \mathrm{Q}$ protein after PNGase treatment in standard conditions $\left(37^{\circ} \mathrm{C}\right.$, overnight, without protease inhibitors) was not possible, although the protein was detectable before digestion 
- detection of a very faint signal elicited by the N388,406Q mutant was possible in experiments only by addition of protease inhibitors and shorter incubation time ( 5 hours) resulted in visible signal in the control incubation (without enzyme), but even here only a very tiny band was detectable in the enzyme treated sample

- besides two glycosylated isoforms of wild-type hEag1, the detection of a nascent unglycosylated protein was not possible

All these observations suggest that the unglycosylated form of the hEag1 protein is unstable and/or very sensitive to proteolytic degradation, although this topic needs to be studied in detail.

\subsection{What is the functional role of $\mathbf{N}$-linked glycosylation of Eag1 potassium channels in native cells?}

Functional studies of Eag1 potassium channels concentrated on different expression systems, but up to now, not much is known about their function in vivo. Nevertheless, there are many indications that Eag1 proteins can play an meaningful role in different cellular events. Important to mention is the oncogenic potential of Eag1. It was clearly shown, that Eag1 is not only expressed in various cell lines of cancerous origin or in tumour tissues, but also favours tumour progression when cells transfected with Eag1 were injected into SCID mice (Pardo et al., 1999). Furthermore, inhibition of Eag1 expression by antisense nucleotides inhibits cell proliferation, indicating a role for Eag1 in tumour progression. This proliferationassociated function of Eag1 is additionally supported by the fact that high levels of Eag1 expression were detected in myoblasts directly before fusion (Occhiodoro et al., 1998). Moreover the function of Eag1 might be associated with endocytosis (Piros et al., 1999) and with the cytoskeleton (Camacho et al., 2000).

Here it is demonstrated that not only hEag1 expressed in CHO cells, but also rat-brain Eag1 undergoes N-linked glycosylation. Besides the regulatory and function in trafficking-support function, N-linked glycosylation is known to play an important role in tumourigenesis (reviewed by Kim et al., 1997 or Orntoft et al., 1999). Changes in glycosylation are more likely to interfere with the later events of invasion and metastasis, such as effects on interaction with ligands in body fluids or in cell membranes of other cells. Thus a possible 
role of N-linked glycosylation of Eag1 could be maintaining a non-malignant phenotype or regulation of proliferation events in native cells.

Up to now neither specific blocker of Eag1 potassium channels, nor knock-out mice are available for the analysis of the Eag1 function. Thus, currently, only speculation about the real role of Eag1 channels and their glycosylation in vivo is possible.

\subsection{Can hEag1 interact with transcription regulators?}

A way to elucidate the function of a protein in vivo is to identify other proteins or macromolecules which bind to it. In general, proteins that interact directly with each other can be expected to participate in related cellular processes.

To identify proteins interacting with hEag1, the yeast two-hybrid system, based on transcriptional activation of reporter genes (Fields and Song, 1989), was used. In two independent screens, a single clone was found, later identified as PIAS1, which putatively interacts with the C-terminus of the human ether á go-go. Two other clones showed only very weak positive signals, and thus their interaction with hEag1 remains questionable.

Surprisingly, none of the already reported Eag1 interaction partners (epsin or calmodulin) were found in the two-hybrid screens. This can be explained by use of only a part of hEag1, and not the entire protein, to identify interactions, although it was shown for epsin to interact with the C-terminus of rat Eag1 only (Piros et al., 1999). On the other hand, the Yeast TwoHybrid System used is based on interactions between two parts of a transcription factor occurring in the cell nucleus. Thus some interactions can be simply overlooked, because of the inefficient or even abolished transport of certain proteins to the yeast cell nucleus. During the last few years new two-hybrid technologies developed, based on other than direct transcriptional activation mechanisms, which enable identification of protein interactions within the cytoplasm and/or plasma membrane. One of them is the hSOS/Ras recruitment system, which relies on the activation of the Ras signalling pathway to rescue a yeast $c d c 25$ mutant (Broder et al., 1998). Anther system, a split-ubiquitin system, is not commonly used because of its unperfect design (Johnsson and Varshavsky, 1994).

PIAS1 (protein inhibitor of activated Stat1) was first identified in a Y2H screen aimed at the identification of potential regulators of Stat1 (reviewed by Shuai, 2000) and shown to inhibit 
STAT1 binding to its consensus response element, thus inhibiting STAT-mediated gene activation. Moreover PIAS proteins have co-regulatory effects on androgen receptor transactivation (Tan et al., 2002) and were also shown to induce p53-activated expression, by interacting with the tetramerisation and C-terminal regulatory domains of p53 (Megidish et al., 2002).

It might seem surprising that PIAS1, a transcriptional activator, functionally interacts with hEag1, an integral membrane protein. However, nuclear localisation of membrane proteins is not an extraordinary finding: Nuclear localisation was shown for EGFR (Epidermal growth factor receptor), which associates with genes and activates sequence-specific gene expression. Recently, nuclear localisation was also reported for a BK channel (calcium- and voltagedependent potassium channel; Hentrich, 2003). The C-terminus of BK channels was found in the nucleus of transfected cells. Moreover the C-terminus of BK channels interacts with BKAP, which is predominantly a nuclear protein, although the function of this interaction is unclear. Finally, an artifactual interaction of PIAS1-hEag1 cannot be completely ruled out.

Eag1 seems to influence proliferation and has oncogenic potential (Pardo et al., 1999). It would be tempting to speculate that interaction of Eag1 (or its C-terminus) with PIAS1 plays a role in the Eag1-related alterations, although it has to be confirmed and characterised in detail. The identification of the hypothetical signalling pathway could give new hints about the function of Eag1 channels in vivo. 


\section{REFERENCES}

Antz C. Bauer T. Kalbacher H. Frank R. Covarrubias M. Kalbitzer HR. Ruppersberg JP. Baukrowitz T. Fakler B. (1999) Control of $\mathrm{K}+$ channel gating by protein phosphorylation: structural switches of the inactivation gate. Nature Structural Biology. 6(2):146-50

Armstrong CM. and Hille B. (1998) Voltage-gated ion channels and electrical excitability. Neuron. 20(3):371-80

Assil IQ. and Abou-Samra AB. (2001) N-glycosylation of CRF receptor type 1 is important for its ligand-specific interaction. American Journal of Physiology - Endocrinology \& Metabolism. 281(5):E1015-21

Bennett E. Urcan MS. Tinkle SS. Koszowski AG. Levinson SR. (1997) Contribution of sialic acid to the voltage dependence of sodium channel gating. A possible electrostatic mechanism. Journal of General Physiology. 109(3):327-43

Bennett ES. (2002) Isoform-specific effects of sialic acid on voltage-dependent $\mathrm{Na}+$ channel gating: functional sialic acids are localized to the S5-S6 loop of domain I. Journal of Physiology. 538(Pt 3):675-90

Bonifacino JS. and Weissman AM. (1998) Ubiquitin and the control of protein fate in the secretory and endocytic pathways. Annual Review of Cell \& Developmental Biology. 14:19-57

Breeden L. and Nasmyth K. Regulation of the yeast HO gene. (1985) Cold Spring Harbor Symposia on Quantitative Biology. 50:643-50

Broder YC. Katz S. Aronheim A. (1998) The ras recruitment system, a novel approach to the study of protein-protein interactions. Current Biology. 8(20):1121-4

Brüggemann A. Pardo LA. Stühmer W. Pongs O. (1993) Ether-a-go-go encodes a voltagegated channel permeable to K+ and Ca2+ and modulated by cAMP. Nature. 365(6445):445-8 
Brüggemann A. Stühmer W. Pardo LA. (1997) Mitosis-promoting factor-mediated suppression of a cloned delayed rectifier potassium channel expressed in Xenopus oocytes. Proceedings of the National Academy of Sciences of the United States of America. 94(2):537-42

Burda P. and Aebi M. (1999) The dolichol pathway of N-linked glycosylation. Biochimica et Biophysica Acta - General Subjects. 1426(2):239-257

Camacho J. Sanchez A. Stuhmer W. Pardo LA. (2000) Cytoskeletal interactions determine the electrophysiological properties of human EAG potassium channels. Pflugers Archiv European Journal of Physiology. 441(2-3):167-74

Castellano A. Chiara MD. Mellstrom B. Molina A. Monje F. Naranjo JR. Lopez-Barneo J. (1997) Identification and functional characterization of a $\mathrm{K}+$ channel alpha-subunit with regulatory properties specific to brain. Journal of Neuroscience. 17(12):4652-61

Chen H. Fre S. Slepnev VI. Capua MR. Takei K. Butler MH. Di Fiore PP. De Camilli P. (1998) Epsin is an EH-domain-binding protein implicated in clathrin-mediated endocytosis. Nature. 394(6695):793-7

Choe S. Kreusch A. Pfaffinger PJ. (1999) Towards the three-dimensional structure of voltage-gated potassium channels. Trends in Biochemical Sciences. 24(9):345-9

Cloos PAC. and Christgau S. (2002) Non-enzymatic covalent modifications of proteins: mechanisms, physiological consequences and clinical applications. Matrix Biology. 21(1):39-52

Conti LR. Radeke CM. Vandenberg CA. (2002) Membrane targeting of ATP-sensitive potassium channel. Effects of glycosylation on surface expression. Journal of Biological Chemistry. 277(28):25416-22

Davidson JS. Flanagan CA. Zhou W. Becker II. Elario R. Emeran W. Sealfon SC. Millar RP. (1995) Identification of N-glycosylation sites in the gonadotropin-releasing hormone receptor: role in receptor expression but not ligand binding. Molecular \& Cellular Endocrinology. $107(2): 241-5$ 
de Souza NF. Simon SM. (2002) Glycosylation affects the rate of traffic of the Shaker potassium channel through the secretory pathway. Biochemistry. 41(38):11351-61

Dijkink L. Hartog A. van Os CH. Bindels RJ. (2002) The epithelial sodium channel (ENaC) is intracellularly located as a tetramer. Pflugers Archiv - European Journal of Physiology. 444(4):549-55

Doering TL. Masterson WJ. Hart GW. Englund PT. (1990) Biosynthesis of glycosyl phosphatidylinositol membrane anchors. Journal of Biological Chemistry. 265(2):611-4

Doyle DA. Morais Cabral J. Pfuetzner RA. Kuo A. Gulbis JM. Cohen SL. Chait BT. MacKinnon R. (1998) The structure of the potassium channel: molecular basis of $\mathrm{K}+$ conduction and selectivity. Science. 280(5360):69-77

Duttweiler HM. (1996) A highly sensitive and non-lethal beta-galactosidase plate assay for yeast. Trends in Genetics. 12(9):340-1

Ellgaard L. Molinari M. Helenius A. (1999) Setting the standards: quality control in the secretory pathway. Science. 286(5446):1882-8

Ellgaard L. and Helenius A. ER (2001) Quality control: towards an understanding at the molecular level. Current Opinion in Cell Biology. 13(4):431-7

Englund PT. (1993) The structure and biosynthesis of glycosyl phosphatidylinositol protein anchors. Annual Review of Biochemistry. 62:121-38

Fields S. and Song O. (1989) A novel genetic system to detect protein-protein interactions. Nature. 340(6230):245-6

Freeman LC, Lippold JJ, and Mitchell KE. (2000) Glycosylation influences gating and pH sensitivity of $\mathrm{I}_{\mathrm{sK}}$. J Membr Biol 177:65-79 
Frings S. Brull N. Dzeja C. Angele A. Hagen V. Kaupp UB. Baumann A. (1998) Characterization of ether-a-go-go channels present in photoreceptors reveals similarity to IKx, a K+ current in rod inner segments. Journal of General Physiology. 111(4):583-99

Gavel Y. and von Heijne G. (1990) Sequence differences between glycosylated and nonglycosylated Asn-X-Thr/Ser acceptor sites: implications for protein engineering. Protein Engineering. 3(5):433-42

Gavrilova-Ruch O. Schonherr K. Gessner G. Schonherr R. Klapperstuck T. Wohlrab W. Heinemann SH. (2002) Effects of imipramine on ion channels and proliferation of IGR1 melanoma cells. Journal of Membrane Biology. 188(2):137-49

Gietz RD. Schiestl RH. Willems AR. Woods RA. (1995) Studies on the transformation of intact yeast cells by the LiAc/SS-DNA/PEG procedure. Yeast. 11(4):355-60

Gong Q. Anderson CL. January CT. Zhou Z. (2002) Role of glycosylation in cell surface expression and stability of HERG potassium channels. Am J physiol Heart Circ Physiol 283: H77-H84

Guarnaccia SP. Shaper JH. Schnaar RL. (1983) Tunicamycin inhibits ganglioside biosynthesis in neuronal cells. Proceedings of the National Academy of Sciences of the United States of America. 80(6):1551-5

Guy HR. and Conti F. (1990) Pursuing the structure and function of voltage-gated channels. Trends in Neurosciences. 13(6):201-6

Hamill OP. Marty A. Neher E. Sakmann B. Sigworth FJ. (1981) Improved patch-clamp techniques for high-resolution current recording from cells and cell-free membrane patches. Pflugers Archiv - European Journal of Physiology. 391(2):85-100

Hanahan D. (1983). Studies on transformation of Escherichia coli with plasmids. J Mol Biol. 166:557-580. 
Hart GW. (1997) Dynamic O-linked glycosylation of nuclear and cytoskeletal proteins. Annual Review of Biochemistry. 66:315-335

He J. Xu J. Castleberry AM. Lau AG. Hall RA. (2002) Glycosylation of beta(1)-adrenergic receptors regulates receptor surface expression and dimerization. Biochemical \& Biophysical Research Communications. 297(3):565-72

Heinemann S. Rettig J. Scott V. Parcej DN. Lorra C. Dolly J. Pongs O. (1994) The inactivation behaviour of voltage-gated K-channels may be determined by association of alpha- and beta-subunits. Journal of Physiology. 88(3):173-80

Helenius A. (1994) How N-linked oligosaccharides affect glycoprotein folding in the endoplasmic reticulum. Molecular Biology of the Cell. 5(3):253-65

Helenius A. and Aebi M. (2001) Intracellular functions of N-linked glycans. Science. 291(5512):2364-9

Henrtich K (2003) BKAP, ein neu entecktes Protein, das mit dem C-Trminus des calciumund spannungsabhängigen Kaliumskanals der Ratte interagiert. Dissertation. Cuvillier Verlag Göttingen

Hoshi N. Takahashi H. Shahidullah M. Yokoyama S. Higashida H. (1998) KCR1, a membrane protein that facilitates functional expression of non-inactivating $\mathrm{K}+$ currents associates with rat EAG voltage-dependent $\mathrm{K}+$ channels. Journal of Biological Chemistry. 273(36):23080-5

Hugnot JP. Salinas M. Lesage F. Guillemare E. de Weille J. Heurteaux C. Mattei MG. Lazdunski M. (1996) Kv8.1, a new neuronal potassium channel subunit with specific inhibitory properties towards Shab and Shaw channels. EMBO Journal. 15(13):3322-31

Huppert SS. Le A. Schroeter EH. Mumm JS. Saxena MT. Milner LA. Kopan R. (2000) Embryonic lethality in mice homozygous for a processing-deficient allele of Notch1. Nature. 405(6789):966-70 
Imperiali B. and O'Connor SE. (1999) Effect of N-linked glycosylation on glycopeptide and glycoprotein structure. Current Opinion in Chemical Biology. 3(6):643-9

James P. Halladay J. Craig EA. (1996) Genomic libraries and a host strain designed for highly efficient two-hybrid selection in yeast. Genetics. 144(4):1425-36

Jenke M. Sanchez A. Monje F. Stühmer W. Weseloh RM. Pardo LA. (2003) C-terminal domains implicated in the functional surface expression of potassium channels. EMBO Journal. 22(3):395-403

Ji IH. Slaughter RG. Ji TH. (1990) N-linked oligosaccharides are not required for hormone binding of the lutropin receptor in a Leydig tumor cell line and rat granulosa cells. Endocrinology. 127(1):494-6

Jiang Y. Lee A. Chen J. Ruta V. Cadene M. Chait BT. MacKinnon R. (2003 A) X-ray structure of a voltage-dependent K+ channel. Nature 423, 33-41

Jiang Y. Ruta V. Chen J. Lee A. MacKinnon R. (2003 B) The principle of gating charge movement in a voltage-dependent K+ channel. Nature 423, 42-48

Johnsson N. and Varshavsky A. (1994) Split ubiquitin as a sensor of protein interactions in vivo. Proceedings of the National Academy of Sciences of the United States of America. 91(22):10340-4

Kasturi L. Eshleman JR. Wunner WH. Shakineshleman SH. (1995) The hydroxy amino acid in an Asn-X-Ser/Thr sequon can influence n-linked core glycosylation efficiency and the level of expression of a cell surface glycoprotein. Journal of Biological Chemistry. 270(24):1475614761

Kerschensteiner D. and Stocker M. (1999) Heteromeric assembly of Kv2.1 with Kv9.3: effect on the state dependence of inactivation. Biophysical Journal. 77(1):248-57 
Khanna R. Myers MP. Laine M. Papazian DM. (2001) Glycosylation increases potassium channel stability and surface expression in mammalian cells. Journal of Biological Chemistry. 276(36):34028-34

Kim YJ. and Varki A. (1997) Perspectives on the significance of altered glycosylation of glycoproteins in cancer. Glycoconjugate Journal. 14(5):569-76

Kornfeld R. and Kornfeld S. (1985) Assembly of asparagine-linked oligosaccharides. Annual Review of Biochemistry. 54:631-64

Kundra R. and Kornfeld S. (1999) Asparagine-linked oligosaccharides protect Lamp-1 and Lamp-2 from intracellular proteolysis. Journal of Biological Chemistry. 274(43):31039-46

Kupershmidt S. Yang T. Chanthaphaychith S. Wang Z. Towbin JA. Roden DM. (2002) Defective human Ether-a-go-go-related gene trafficking linked to an endoplasmic reticulum retention signal in the C terminus. Journal of Biological Chemistry. 277(30):27442-8

Lecain E. Sauvaget E. Crisanti P. Van den Abbeele T. Huy PTB. (1999) Potassium channel ether a go-go mRNA expression in the spiral ligament of the rat. Hearing Research. 133 (1-2): 133-138

Lechner J. and Wieland F. (1989) Structure and biosynthesis of prokaryotic glycoproteins. Annual Review of Biochemistry. 58:173-94

Ludwig J. Terlau H. Wunder F. Bruggemann A. Pardo LA. Marquardt A. Stuhmer W. Pongs O. (1994) Functional expression of a rat homologue of the voltage gated either a go-go potassium channel reveals differences in selectivity and activation kinetics between the Drosophila channel and its mammalian counterpart. EMBO Journal. 13(19):4451-8

Ludwig J. Owen D. Pongs O. (1997) Carboxy-terminal domain mediates assembly of the voltage-gated rat ether-a-go-go potassium channel. EMBO Journal. 16(21):6337-45

MacKinnon R. (1991) New insights into the structure and function of potassium channels. Current Opinion in Neurobiology. 1(1):14-9 
Maniatis T. and Efstratiadis A. (1980). Fractionation of low molecular weight DNA or RNA inpolyacrylamide gels containing 98\% formamide or $7 \mathrm{M}$ urea. Methods in Enzymology. 65:299-305.

Marty A. and Neher E. (1983) Tight seal whole cell recording. Single Channel Recording. Editors: Sakmann B., Neher E., Plenum NY.

Megidish T. Xu JH. Xu CW. (2002) Activation of p53 by protein inhibitor of activated Stat1 (PIAS1). Journal of Biological Chemistry. 277(10):8255-9

Messner P. (1997) Bacterial glycoproteins. Glycoconjugate Journal. 14(1):3-11

Meyer R. and Heinemann SH. (1998) Characterization of an eag-like potassium channel in human neuroblastoma cells. Journal of Physiology. 508(Pt 1):49-56

Meyer R. Schonherr R. Gavrilova-Ruch O. Wohlrab W. Heinemann SH. (1999) Identification of ether a go-go and calcium-activated potassium channels in human melanoma cells. Journal of Membrane Biology. 171(2):107-15

Molinari M. and Helenius A. (2000) Chaperone selection during glycoprotein translocation into the endoplasmic reticulum. Science. 288(5464):331-3

Morais Cabral JH. Lee A. Cohen SL. Chait BT. Li M. Mackinnon R. (1998) Crystal structure and functional analysis of the HERG potassium channel $\mathrm{N}$ terminus: a eukaryotic PAS domain. Cell. 95(5):649-55

Moreno J. Cruz-Vera LR. García-Villegas MR. Cereijido M. (2002) Polarized Expression of Shaker Channels in Epithelial Cells. Journal of Membrane Biology 190(3):175-187

Nagaya N. and Papazian DM. (1997) Potassium channel alpha and beta subunits assemble in the endoplasmic reticulum. Journal of Biological Chemistry. 272(5):3022-7 
Nosjean O. Briolay A. Roux B. (1997) Mammalian gpi proteins - sorting, membrane residence and functions Biochimica et Biophysica Acta -Reviews on Biomembranes. 1331(2):153-186

Occhiodoro T. Bernheim L. Liu JH. Bijlenga P. Sinnreich M. Bader CR. Fischerlougheed J. (1998) Cloning of a human ether-a-go-go potassium channel expressed in myoblasts at the onset of fusion. FEBS Letters. 434(1-2):177-182

Ogden RC. and Adams DA. (1987). Electrophoresis in agarose and acrylamide gels. Methods in Enzymology, Academic Press, Inc., San Diego, USA. 152:61-87

Olsen O. Borriss R. Simon O. Thomsen KK. (1991) Hybrid Bacillus (1-3,1-4)-betaglucanases: engineering thermostable enzymes by construction of hybrid genes. Molecular \& General Genetics. 225(2):177-85

Orntoft TF. and Vestergaard EM. (1999) Clinical aspects of altered glycosylation of glycoproteins in cancer. Electrophoresis. 20(2):362-71

Ou WJ. Cameron PH. Thomas DY. Bergeron JJ. (1993) Association of folding intermediates of glycoproteins with calnexin during protein maturation. Nature. 364(6440):771-6

Papazian DM. Timpe LC. Jan YN. Jan LY. (1991) Alteration of voltage-dependence of Shaker potassium channel by mutations in the S4 sequence. Nature. 349(6307):305-10

Pardo LA. Bruggemann A. Camacho J. Stühmer W. (1998) Cell cycle-related changes in the conducting properties of r-eag K+ channels. Journal of Cell Biology. 143(3):767-75

Pardo LA. del Camino D. Sanchez A. Alves F. Bruggemann A. Beckh S. Stühmer W. (1999) Oncogenic potential of EAG K+ channels. EMBO Journal. 18(20): 5540-5547

Petrecca K. Atanasiu R. Akhavan A. Shrier A. (1999) N-linked glycosylation sites determine HERG channel surface membrane expression. Journal of Physiology. 515 (1):41-8 
Piros ET. Shen L. Huang XY. (1999) Purification of an EH domain-binding protein from rat brain that modulates the gating of the rat ether-a-go-go channel. Journal of Biological Chemistry. 274(47):33677-83

Puck TT et al. (1958) Genetics of somatic mammalian cells III. Long-term cultivation of euploid cells from human and animal subjects. J. Exp. Med. 108:945-956

Rettig J. Heinemann SH. Wunder F. Lorra C. Parcej DN. Dolly JO. Pongs O. (1994) Inactivation properties of voltage-gated $\mathrm{K}+$ channels altered by presence of beta-subunit. Nature. 369(6478):289-94

Rettinger J. Aschrafi A. Schmalzing G. (2000) Roles of individual N-glycans for ATP potency and expression of the rat P2X1 receptor. Journal of Biological Chemistry. 275(43):33542-7

Rho S. Lee HM. Lee K. Park C. (2000) Effects of mutation at a conserved N-glycosylation site in the bovine retinal cyclic nucleotide-gated ion channel. FEBS Letters. 478(3):246-52

Rudd PM. Wormald MR. Stanfield RL. Huang M. Mattsson N. Speir JA. DiGennaro JA. Fetrow JS. Dwek RA. Wilson IA. (1999) Roles for glycosylation of cell surface receptors involved in cellular immune recognition. Journal of Molecular Biology. 293(2):351-66

Rudd PM. Elliott T. Cresswell P. Wilson IA. Dwek RA. (2001) Glycosylation and the immune system. Science. 291(5512):2370-6

Rutishauser U. (1996) Polysialic acid and the regulation of cell interactions. Current Opinion in Cell Biology. 8(5):679-84

Saiki, R.K., Gelfand, D.H., Stoffel, S., Scharf, S.J., Higuchi, R., Horn, G.T., Mullis, K.B. and Erlich, H.A. (1988). Primer-directed enzymatic amplification of DNA with a thermostable DNA polymerase. Science 239:487-491 
Saito Y. Tetsuka M. Yue L. Kawamura Y. Maruyama K. (2003) Functional role of N-linked glycosylation on the rat melanin-concentrating hormone receptor 1. FEBS Letters. 533(13):29-34

Sambrook J. Fritsch EF. Maniatis T. (1989) Molecular cloning: a laboratoty manual (2 ${ }^{\text {nd }}$ edition). Cold Spring Habour, New York, USA.

Santacruz-Toloza L. Huang Y. John SA. Papazian DM. (1994) Glycosylation of shaker potassium channel protein in insect cell culture and in Xenopus oocytes. Biochemistry. 33(18):5607-13

Satler CA. Vesely MR. Duggal P. Ginsburg GS. Beggs AH. (1998) Multiple different missense mutations in the pore region of HERG in patients with long QT syndrome. Human Genetics. 102(3):265-272

Sato C. Ueno Y. Asai K. Takahashi K. Sato M. Engel A. Fujiyoshi Y. (2001) The voltagesensitive sodium channel is a bell-shaped molecule with several cavities. Nature. 409(6823):1047-51

Scannevin RH. and Trimmer JS. (1997) Cytoplasmic domains of voltage-sensitive $\mathrm{K}^{+}$ channels involved in mediating protein-protein interactions. Biochemical \& Biophysical Research Communications. 232(3):585-9

Schonherr R. Lober K. Heinemann SH. (2000) Inhibition of human ether a go-go potassium channels by $\mathrm{Ca}\left(2^{+}\right) /$calmodulin. EMBO Journal. 19(13):3263-71

Schwalbe RA. Wang Z. Wible BA. and Brown AM. (1995) Potasium channel structure and function as reported by a single glycosylation sequon Journal of Biological Chemistry 270:15336-15340

Shakin-Eshleman SH. Spitalnik SL. Kasturi L. (1996) The amino acid at the X position of an Asn-X-Ser sequon is an important determinant of N-linked core-glycosylation efficiency. Journal of Biological Chemistry. 271(11):6363-6 
Sharon N. and Lis H. (1972) Lectins: cell-agglutinating and sugar-specific proteins. Science. 177(53):949-59

Shi G. Nakahira K. Hammond S. Rhodes KJ. Schechter LE. Trimmer JS. (1996) Beta subunits promote $\mathrm{K}+$ channel surface expression through effects early in biosynthesis. Neuron. 16(4):843-52

Shi G. Trimmer JS. (1999) Differential asparagine-linked glycosylation of voltage-gated K+ channels in mammalian brain and in transfected cells. Journal of Membrane Biology. 168(3):265-73

Shuai K. (2000) Modulation of STAT signaling by STAT-interacting proteins. Oncogene. 19(21):2638-44,

Sokolova O. Kolmakova-Partensky L. Grigorieff N. (2001) Three-dimensional structure of a voltage-gated potassium channel at $2.5 \mathrm{~nm}$ resolution. Structure. 9(3):215-20

Spector DL. Golgman RD. Leinwand LA. (1998) Cells. A Laboratory Manual Cold Spring Harbor Laboratory Press.

Spiro RG. (1973) Glycoproteins. Advances in Protein Chemistry. 27:349-467

Spiro RG. (2002) Protein glycosylation: nature, distribution, enzymatic formation, and disease implications of glycopeptide bonds. Glycobiology. 12(4):43R-56R

Stühmer W. Conti F. Suzuki H. Wang XD. Noda M. Yahagi N. Kubo H. Numa S. (1989) Structural parts involved in activation and inactivation of the sodium channel. Nature. 339(6226):597-603

Stühmer W. (1992) Electrophysiological Recording from Xenopus Oocytes. Methods in Enzymology. Ion channels vol 207. Edited by: Rudy B., Iverson LE. Academic press, New York, USA. 
Tan JA. Hall SH. Hamil KG. Grossman G. Petrusz P. French FS. (2002) Protein inhibitors of activated STAT resemble scaffold attachment factors and function as interacting nuclear receptor coregulators. Journal of Biological Chemistry. 277(19):16993-7001

Terlau H. and Stuhmer W. (1998) Structure and function of voltage-gated ion channels. Naturwissenschaften. 85(9):437-44

Thornhill WB. Wu MB. Jiang X. Wu X. Morgan PT. and Margiotta JF. (1996) Expression of Kv 1.1 delayed rectifier channels in Lec mutant Chinese hamster ovary cell lines reveals a role for sialidation in channel function. Journal of Biological Chemistry. 271:19093-19098

Tkacz JS. and Lampen O. (1975) Tunicamycin inhibition of polyisoprenyl Nacetylglucosaminyl pyrophosphate formation in calf-liver microsomes. Biochemical \& Biophysical Research Communications. 65(1):248-57

Torres GE. Egan TM. Voigt MM. (1998) N-Linked glycosylation is essential for the functional expression of the recombinant P2X2 receptor. Biochemistry. 37(42):14845-51

Towbin H. Staehelin T. Gordon J. (1979) Electrophoretic transferof proteins from polyacrylamide gels to nitrocellulose sheets: Procedure and some applications. PNAS USA 76: 4350-4354

Van den Steen P Rudd PM. Dwek RA. Opdenakker G. (1998) Concepts and Principles of OLinked Glycosylation. Critical Reviews in Biochemistry and Molecular Biology, 33(3):151208

Varki A. (1993) Biological roles of oligosaccharides: all of the theories are correct. Glycobiology. 3(2):97-130

Venter JC. Adams MD. Myers EW. Li PW. Mural RJ. Sutton GG. Smith HO. Yandell M. Evans CA. Holt RA. et al. (2001) The sequence of the human genome. Science. 291(5507):1304-+ 
Vosseller K. Sakabe K. Wells L. Hart GW. (2002) Diverse regulation of protein function by O-GlcNAc: a nuclear and cytoplasmic carbohydrate post-translational modification. Current Opinion in Chemical Biology. 6(6):851-857

Warmke J. Drysdale R. Ganetzky B. (1991) A distinct potassium channel polypeptide encoded by the Drosophila eag locus. Science. 252(5012):1560-2

Warmke JW. and Ganetzky B. (1994) A family of potassium channel genes related to eag in Drosophila and mammals. Proceedings of the National Academy of Sciences of the United States of America. 91(8):3438-42

Wells L. Vosseller K. Hart GW. (2001) Glycosylation of nucleocytoplasmic proteins: Signal transduction and O-GlcNAc. Science. 291(5512):2376-2378

Wickman KD. and Clapham DE. (1995) G-protein regulation of ion channels. Current Opinion in Neurobiology. 5(3):278-85

Wilson GF. Wang Z. Chouinard SW. Griffith LC. Ganetzky B. (1998) Interaction of the K channel beta subunit, Hyperkinetic, with eag family members. Journal of Biological Chemistry. 273(11):6389-94

Wilson IBH. (2002) Glycosylation of proteins in plants and invertebrates. Current Opinion in Structural Biology. 12(5):569-577

Wu CF. Ganetzky B. Haugland FN. Liu AX. (1983) Potassium currents in Drosophila: different components affected by mutations of two genes. Science. 220(4601):1076-8

Wulfsen I. Hauber HP. Schiemann D. Bauer CK. Schwarz JR. (2000) Expression of mRNA for voltage-dependent and inward-rectifying $\mathrm{K}$ channels in GH3/B6 cells and rat pituitary. Journal of Neuroendocrinology. 12(3):263-72

Yusuf HK. Pohlentz G. Sandhoff K. (1983) Tunicamycin inhibits ganglioside biosynthesis in rat liver Golgi apparatus by blocking sugar nucleotide transport across the membrane vesicles. 
Proceedings of the National Academy of Sciences of the United States of America. 80(23):7075-9

Zachara NE. and Hart GW. (2002) The emerging significance of O-GlcNAc in cellular regulation. Chemical Reviews. 102(2):431-8

Zerangue N. Schwappach B. Jan YN. Jan LY. (1999) A new ER trafficking signal regulates the subunit stoichiometry of plasma membrane K(ATP) channels. Neuron. 22(3):537-48

Zhang Y. Hartmann HA. Satin J. (1999) Glycosylation influences voltage-dependent gating of cardiac and skeletal muscle sodium channels. Journal of Membrane Biology. 171(3):195-207

Zhou Z. Gong Q. Ye B. Fan Z. Makielski JC. Robertson GA. January CT. (1998 A) Properties of HERG channels stably expressed in HEK 293 cells studied at physiological temperature. Biophysical Journal. 74(1):230-41

Zhou Z. Gong Q. Epstein ML. January CT. (1998 B) HERG channel dysfunction in human long QT syndrome. Intracellular transport and functional defects. Journal of Biological Chemistry. 273(33):21061-6 


\section{Acknowledgements}

The completion of this thesis has been made possible only because I had the fortune of counting with the support of many people. It is very difficult to name them all and before acknowledging anybody I would like to express them my best gratitude.

From the ones I can individually thank I would like to start with acknowledging Prof Dr. Walter Stühmer for giving me the opportunity to work in his department, for his interest and support. For being responsible in a big extent for the prevalence of a fantastic and stimulating research environment and a comfortable working atmosphere. Thanks as well for all the help he provided me.

Prof Dr. Jahn and Dr. Rickmann, members of my PhD committee, my thanks for advice and helpful discussion.

A special thank to Dr. Luis Pardo, for his invaluable help because without it this work might not be finished. Especial thanks for the 'ach so!', 'fuxia' and many other things that made me enjoy even in the difficult times. Despite his multiple preoccupations, he devoted much of his time to supervise this project right to the end. I hope he finds this dissertation a motive of pride, satisfaction and encouragement in his own career.

Dr. Francisco Monje, my enormous appreciation for the great work and enormous help on the electrophysiology experiments. I really value your patience while explaining and answering millions of questions (most of them more than once).

I am particularly very grateful to Dr. Synnöve Beckh for the assistance and corrections made to improve the quality of this dissertation.

I'd like to thank former and actual colleagues in our department, especially Anna Boccacio, Jasmin Djannatian, Rocio Finol, Mark Jenke, Sabine Martin, Johen Müller, Nadja Osterberg, Araceli Sanchez, Claudia Weber, and all the others for their collaboration and solidarity; for their friendship, help, encouragement and great support. A special thank to Rocio and Johen for the best coffee of the world.

I am obliged to Dr. Pilar Correro, Sylvia, Noelia, David and Diego for their help and cooperation in the $\mathrm{Y} 2 \mathrm{H}$ project. Also for their friendship and support during my wonderful time in Oviedo (Spain).

For precious help, organisation and professional support I want to recognize Helgard Rinnert, Ute Rust, Barbara Scheufler, Uschi Kutzke, Mona Heinemann, Victor Diaz, Mari Tufekcic, Barbara Boczek, Wolfram Lessner, Jörg Schischkoff, Ralph Schliephake, and many other people. I would like to express my sincere gratefulness to all my friends from different parts of the world I did not mention before, especially to Dagmara Boinska for invaluable friendship and help to overcome the difficult periods.

Finally, extra special thanks to my family, my mother, my father, my brother, my sister-inlaw Beata and mostly to my husband Mario, for their support and love. Thank for all what you have done for me.

\section{Dziekuje!}




\section{Curriculum vitae}

\section{Personal details:}

Name: Napp Joanna

Date of birth: 7th January 1974

Place of birth: Bydgoszcz, Poland

Nationality: Polish

\section{Educational background:}

1980-1992 Primary and Secondary school, Bydgoszcz, Poland.

1992 High School Diploma.

1992-1998 Studies of Biology, Nicolaus Copernicus University, Faculty of Biology and Earth Science, Torun, Poland.

1998/1999 Diploma work at the Institut for Human Genetics, Göttingen

1998 Master of Science. Thesis: "Maus TSGm1 gene- its structure and haploid expression”.

2000-2003 PhD studies at the Max Planck Institute for experimental Medicine, Göttingen, Department of Molecular Biology of Neuronal Signals, under the supervision of Prof. Dr. W. Stühmer. 